\title{
The Growth of Thin Lubricating Films of Plant Oils
}

A dissertation submitted for the fulfilment of the requirements for the degree of Doctor of Philosophy

December 2012

Wenhsi Chua

Tribology Laboratory

School of Mechanical and Chemical Engineering University of Western Australia 
This research was supported by the School of Mechanical and Chemical Engineering, University of Western Australia. The doctoral candidate is a recipient of the University Postgraduate Scholarship and the Samaha Research Top-Up Scholarship. 


\begin{abstract}
The extreme conditions of high pressure and shear imposed in a lubricated sliding contact could promote tribochemical reactions and polymerization over long sliding distances. These chemical changes are likely to alter the film thickness and friction. Unsaturated plant oils inherently possess two characteristics that would favour the process of polymerization at the lubricant-surface interface - poor oxidation stability and a high affinity with metals. Boundary films formed by plant oils could possibly polymerize and thicken when they are subjected to long periods of lubricated sliding. A new tribometer, capable of simultaneously measuring film thickness, friction and wear was developed to test this hypothesis. The instrument is capable of measuring film thickness measurements down to $1 \mathrm{~nm}$ by employing a modified capacitance method.
\end{abstract}

In all, thirteen refined and unrefined plant oils were tested. The results revealed for the first time, that boundary films of some plant oils can grow to thicknesses much greater than their adsorbed molecular heights. Some films grew as much as 25 times in thickness (unrefined canola oil), while others remained roughly unchanged (flaxseed and olive oil), or grew slightly and then collapsed during the test (safflower oil). It was initially expected that the film growth rate could be correlated to the degree of unsaturation of the oils. However, results from gas chromatography mass spectroscopy (GCMS) analysis revealed little evidence of such correlation. It follows that minor components, particularly in the unrefined oils, might have had an influence on the film growth behaviour.

To further investigate this, surface-enhanced Raman spectroscopy (SERS) was used to study and compare the differences in the chemical nature of the boundary films formed by refined and unrefined canola oil. This is the first time that metallic surfaces lubricated by plant oils have been studied using this technique. The results of this work provided strong evidence 
that fatty acids were liberated from the triglyceride structure during sliding to form a fatty acid soap layer on the silver surface. The study also revealed that the fatty acid chains of the unrefined canola oil were more disordered and most likely in a gauche conformation, while that of the refined canola oil were tightly packed and oriented perpendicular to the surface. Using inductively-coupled plasma mass spectroscopy (ICP-MS), comparatively higher levels of phosphorus, were also found in the unrefined canola oil and unrefined macadamia oil samples, both of which had experienced greater film growth. It is believed that the phosphorus found originated from phospholipids in the unrefined oils, could have been directly or indirectly related to the film growth observed. Its role and the mechanism in which it affects film growth, however, remain to be elucidated. 
For my mother, Emily Lee $\Leftrightarrow$

In loving memory of my grandparents,

Tan Lee Siang and Lee Yong Ker 


\section{Contents}

Preface

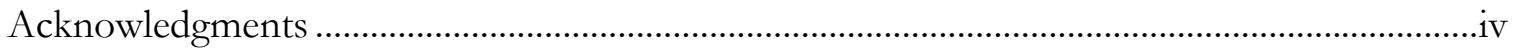

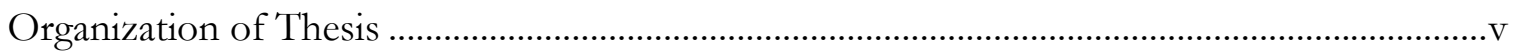

Journal Publications and Conference Presentations Arising from this Work ............................vii

Statement of Candidate's Contribution...................................................................................... viii

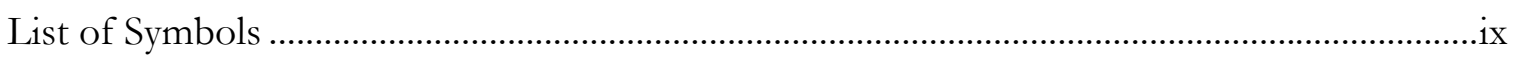

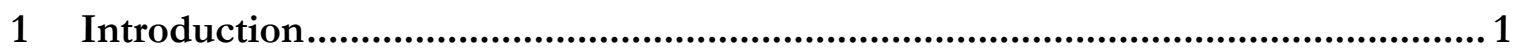

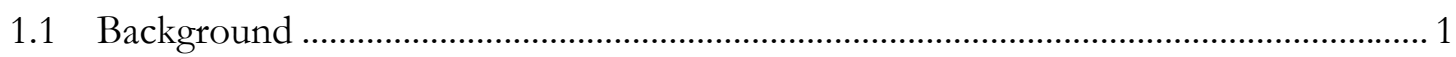

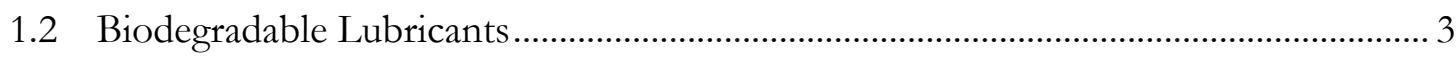

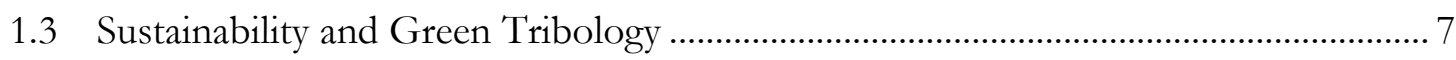

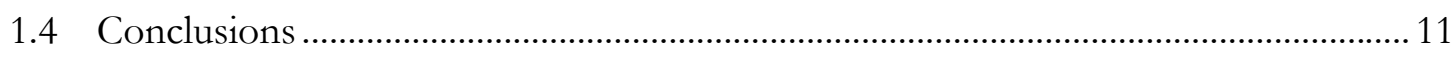

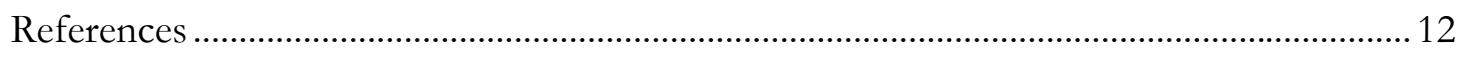

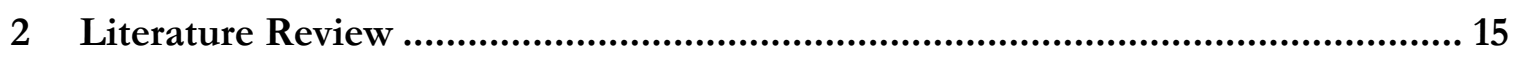

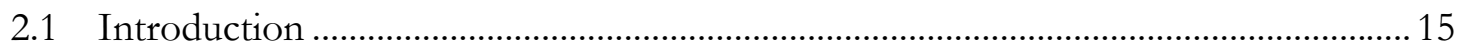

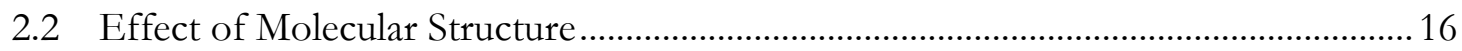

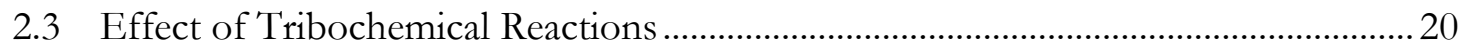

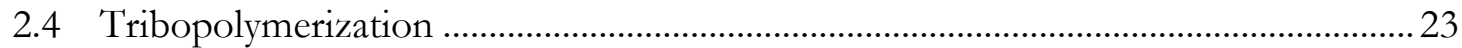

2.5 Limitations of Conventional Boundary Lubrication Study Methods ..........................2. 25

2.6 Film Thickness Measurement Techniques and Their Limitations ...............................26

2.6.1 Optical Interferometry Method........................................................................ 27

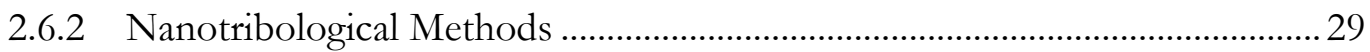

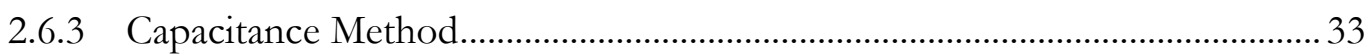

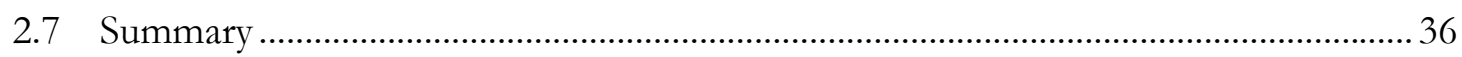

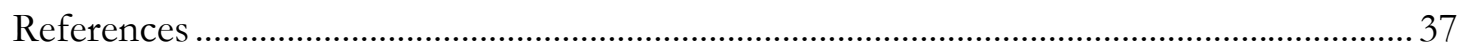


3 Capacitance Method for Film Thickness Measurement

3.1 Introduction

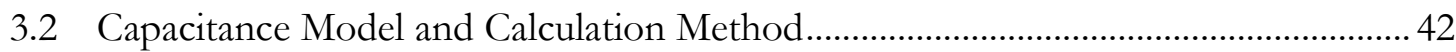

3.2.1 Capacitance Contribution Within the Apparent Area of Contact................... 43

3.2.2 Capacitance Contribution Outside the Apparent Area of Contact................. 46

3.2.3 Conversion of Capacitance Values to Film Thickness Values.......................... 47

3.3 Prediction of the Dielectric Constant, $\varepsilon$ at High Pressures ............................................. 48

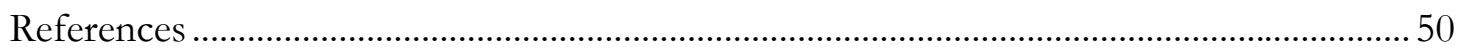

4 Development of the Contact Impedance Tribometer.......................................... 52

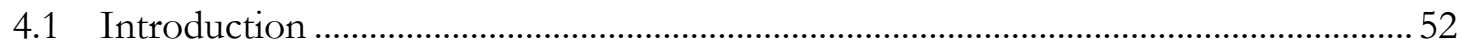

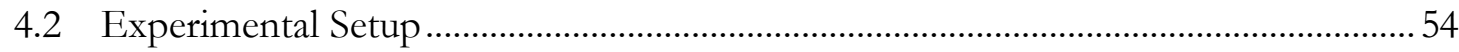

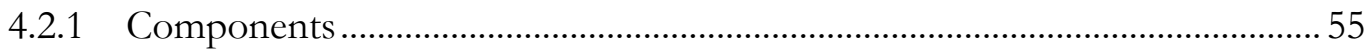

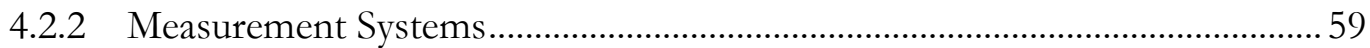

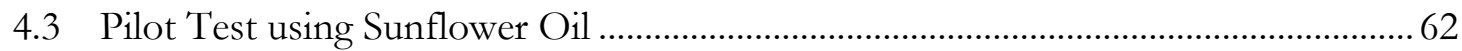

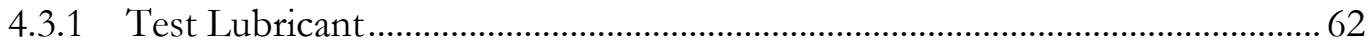

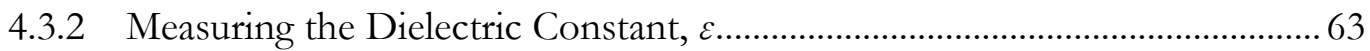

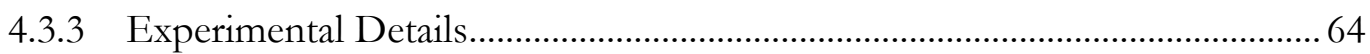

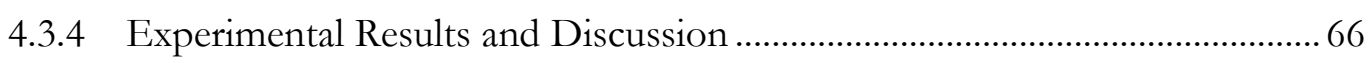

4.4 Discussion on the Accuracy and Limitations of the Method ....................................... 70

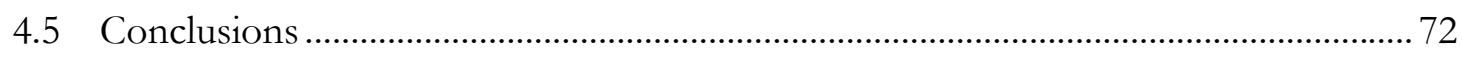

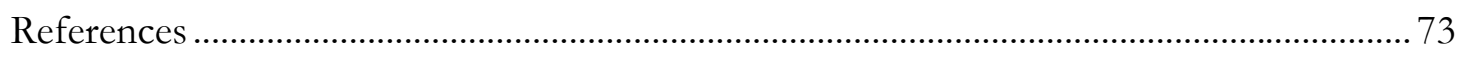

5 The Growth of Thin Lubricating Films of Plant Oils........................................ 74

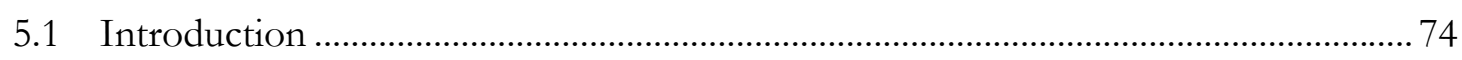

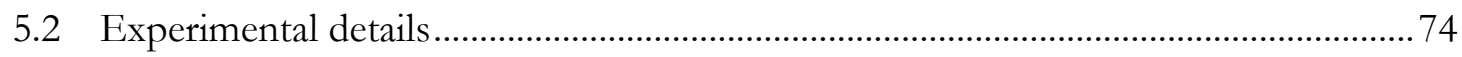

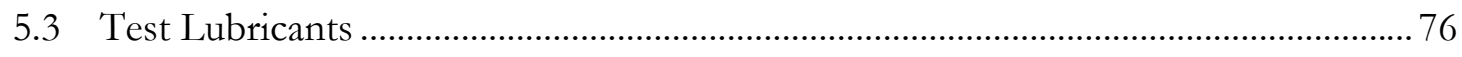

5.3.1 Physical Properties ............................................................................................ 76

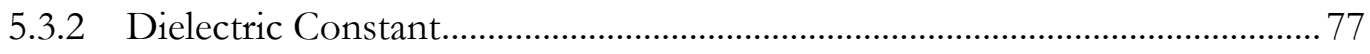

5.3.3 Fatty Acid Composition and Free Fatty Acids (FFAs) ...................................... 81

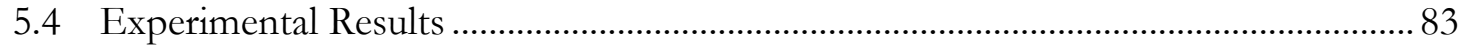

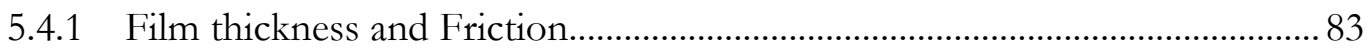

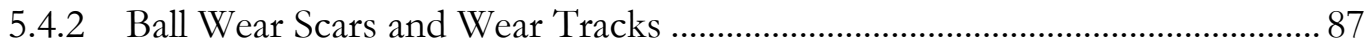

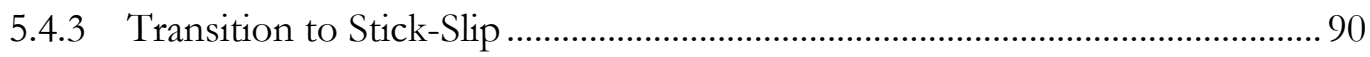

5.4.4 Viscoelastic-like Behaviour at 'Stop' .................................................................. 92 
5.4.5 Effect of Sudden Changes in Sliding Speeds.

5.5 Discussion

5.6 Conclusions .99

Additional Material: Elemental Composition using ICP-MS 100

References 102

6 The Chemical Nature of Canola Oil Boundary Films 104

6.1 Introduction 104

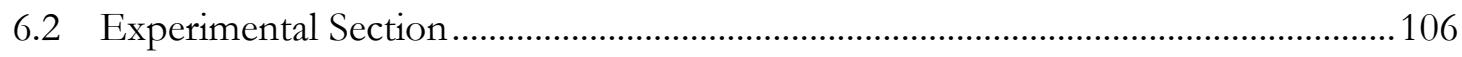

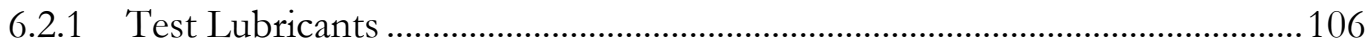

6.2.2 Tribological Test and Sample Preparation.......................................................106

6.2.3 Surface Enhanced Raman Spectroscopy (SERS)............................................. 108

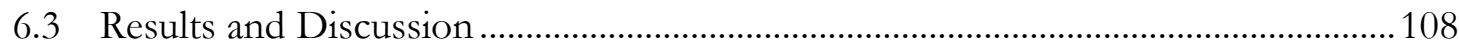

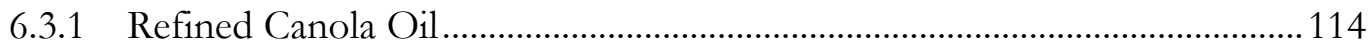

6.3.2 Unrefined Canola Oil......................................................................................116

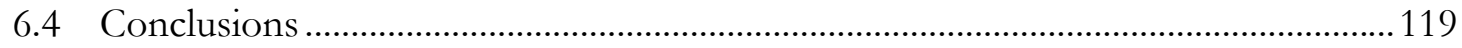

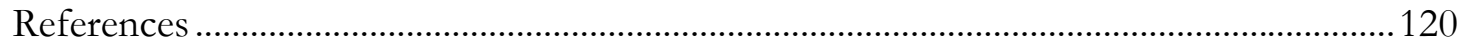

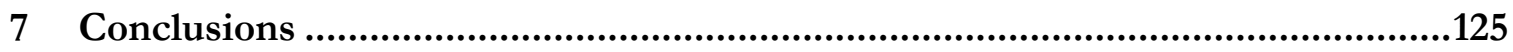

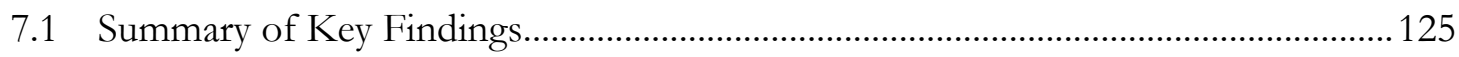

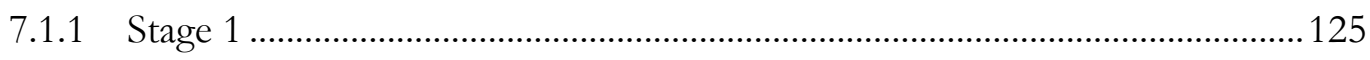

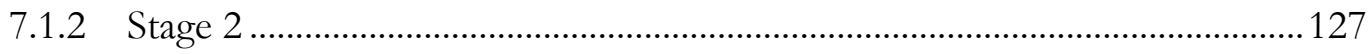

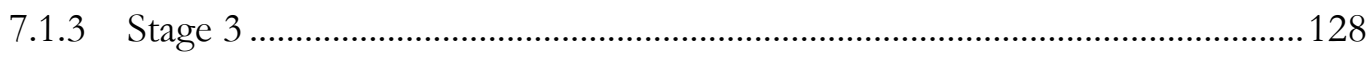

7.2 Recommendations for Future Work …………..................................................130

7.2.1 Effects of Varying Sliding Speeds on the Film Thickness and Friction......130

7.2.2 Quantification of Minor Components and Tribological Test .......................130

7.2.3 Validation of the Effects of Minor Components in Film Growth ...............131

7.2.4 Surface-sensitive Spectroscopy Analysis .............................................................132

Appendix A: Original Film Thickness and Friction Traces .................................133

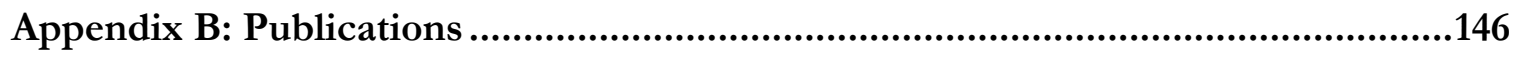




\section{Preface}

This $\mathrm{PhD}$ thesis is a culmination of the work carried out at the Tribology Laboratory of the University of Western Australia from 2007 to 2012. It is a concise compilation of the thought processes, key developments and findings of the work in the last six years.

My interest in the mechanisms of boundary lubrication of fatty acids stemmed from reading the classical work of Bowden and Tabor (The Friction and Lubrication of Solids, 1950). One particular area of interest is the change of friction with time under boundary lubrication conditions. The cause of this change may be obvious in some cases where the real and apparent area of contacts increases due to wear. In other cases, however, frictional changes were also observed with little or no wear occurring. Logic suggests that this change in friction must then be due to either a change in the surface properties, or more likely, a change in the shear strength of the boundary film. This change in the shear strength of the boundary film is undoubtedly the work of tribochemical reactions that take place during sliding contact.

The spark and hypothesis for this work came after reading M.J. Furey's work on tripolymerization, a concept of reducing wear and friction by the formation of polymeric films on rubbing surfaces. These polymeric films are formed by compounds that readily polymerize under extreme conditions of pressure and heat. This could apply to polyunsaturated fatty acids and plants oils and for the same reason, they have been used commonly as drying oils, coatings, resins, adhesives and hardeners. The poor oxidation stability of unsaturated fatty acids and plant oils, coupled with their affinity with metallic surfaces would make them ideal for generating tribopolymerized boundary films. The extreme conditions of high shear, pressure and temperature in a sliding contact could cause the boundary film to thicken over 
time, thereby providing a thick protective layer between rubbing surfaces against wear and may cause frictional properties to alter.

In order to investigate this, it is necessary to measure the film thickness and friction in situ, while the triblogical tests are conducted. Film thickness measurement of such ultra-thin lubricant films requires sensitive techniques, such as the widely used optical interferometry method, which can measure film thicknesses down to the nanometer level. However, they cannot do so for extended periods due to their sensitivity to wear. Thus, the observation of boundary films using the optical interferometry methods are generally restricted to short periods and investigations into the growth of boundary films are therefore rare. It became clear that the only way to test the hypothesis was to develop a new instrument.

The old capacitance method first developed by Crook in 1958, was revisited. The capacitance method offers several advantages over the optical interferometry method. Unlike the optical interferometry method, the capacitance method requires the two rubbing surfaces to be made of metal. Hence, the tribochemical effects of rubbing two lubricated metallic surfaces can be studied. The instrument is also not as sensitive to the effects of wear, as compared to the optical method. Severe wear conditions, however, could render the capacitance method ineffective due to short circuiting caused by large amount of wear particles entrained in the contact. The sensitivity of the capacitance measurement, by nature, increases when the film thickness decreases, which is ideal for boundary film lubrication studies. The only drawback of the method is that the method depends on the knowledge of the lubricant's dielectric constant in the area of contact, and the difficulty in measuring the actual temperature in the area of contact causes uncertainties in the dielectric constant.

Almost two years of this research journey were spent on the design and development of this new instrument, half of which was spent on improving the accuracy and precision of the instrument. The effort and time spent to develop the new instrument to an acceptable 
degree of precision and accuracy was well justified, as the newly developed tribometer was able to report tribological behaviours of plant oil boundary films never seen before. Using thirteen refined and unrefined plant oils, convincing evidence of the phenomena of boundary film growth were produced. Attempts were made to study these boundary films via surfaceenhanced Raman spectroscopy, a spectroscopic technique that is very sensitive to the chemical signature of the adsorbed layers of the surface films. They provided further insight into the chemical nature and molecular structure of plant oil boundary films. However, the mechanisms of this growth remain uncertain and elusive. 


\section{Acknowledgments}

Firstly, I would like to thank my supervisor, Professor Gwidon Stachowiak, for his support and guidance throughout this candidature. I would also like to thank my colleagues in the Tribology Laboratory - A/Prof Grazyna Stachowiak, for her advice regarding my work, and Mr. Mobin Salasi, for his friendship. I would like to thank the School's workshop highlyskilled technicians, Mr. Mike Reid and Mr. Rienier de Lange, for without their patience to my 'trial and error approach' at times, the successful development and manufacture of the new test rig would not have been possible. Thanks also go to the friendly and helpful staff of Metabolomics Australia, particularly, Ms. Ricarda Fenske and Dr. Matthew Timmins, who performed the GCMS analysis of the plant oils. I also owe my gratitude to Mr Peter Chapman of the Department of Chemistry at Curtin University of Technology. His guidance on the use of the FTIR and Raman spectrometers was instrumental in the final work presented in this thesis.

For the people whom, from the beginning, had encouraged me to return to Australia to pursue this $\mathrm{PhD}$, I would like to thank Dr Peter Ridley, my course coordinator during my bachelors degree course at Queensland University of Technology (QUT) and Professor Will Scott, whom, with his Scottish humour, had made attending Tribology classes a joy. Last but not least, to my mother, Emily Lee, who has always been there for me, even though she is thousands of miles away. I will forever be indebted to her. 


\section{Organization of Thesis}

The thesis has been structured in the same order in which the research has been conducted.

The work following the literature review can be divided into three distinct stages, as shown in the flow chart below. These three stages are presented in three published works respectively, which have been edited and incorporated in the form of separate chapters as part of this thesis.

In the introductory first chapter, a brief historical account of lubricants, and the technical and economic challenges facing plant-oil based lubricants today are presented It also discusses the sustainability of bio-based lubricants as a viable alternative to mineral oils. In Chapter 2, a literature review of our current understanding of the molecular structure of plant oils and its effects on tribological properties are presented. It highlights the tribological results reported by others and the gaps in our understanding of plant oil lubrication. A brief review of the current techniques and state-of-the-art instrumentation used in the study of boundary lubrication is also presented.

Stage 1

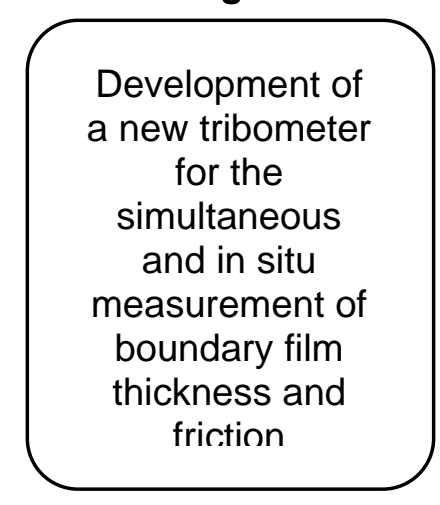

Chapter $3 \& 4$
Stage 2

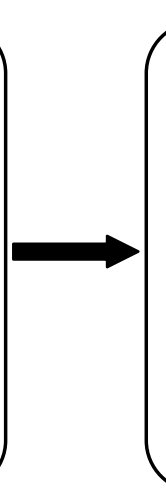

Experimental investigation of the effects of sliding distance on the thickness and friction of plant oil boundary films
Stage 3

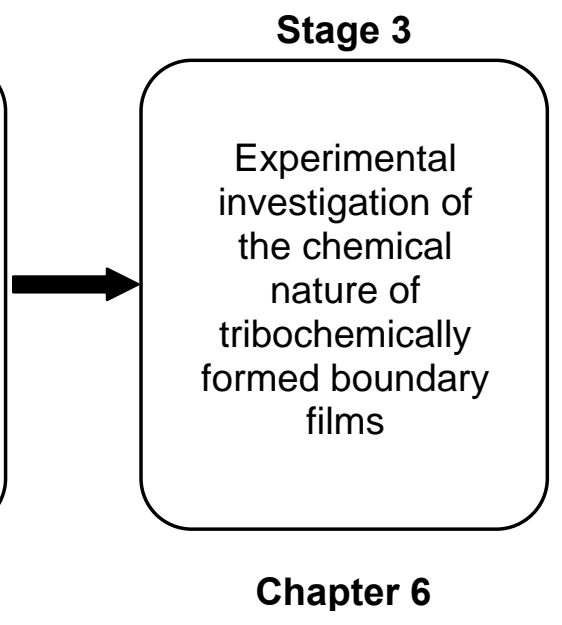


The capacitance method for film thickness measurement was chosen for the development of the new instrument in Stage 1. The theoretical formulation for the capacitance method is presented in Chapter 3. The capacitance method has been derived from first principles by assuming a capacitance model, and then inversely calculating the film thickness from capacitance values. An empirical method for the calculation of the dielectric constant at the area of contact has also been shown. In Chapter 4, the design of the new experimental rig, called the Contact Impedance Tribometer, based on the capacitance method is described. The instrument is capable of simultaneously measuring friction, wear and film thickness down to $1 \mathrm{~nm}$ in situ. Results from the pilot test conducted using refined sunflower oil are also presented.

The work and results presented in the Chapter 5 represents the core of this research. The experiment was extended to nine unrefined oils and three refined oils. Densities, viscosities refractive indices, dielectric constants of the plant oils were measured prior to the tribological tests. The oils were each subjected to a sliding period of 18 hours, where their film thickness and friction traces were recorded simultaneously. The objective of the work was to determine if there is a relationship between the film growth behaviours, and their physical properties and fatty acid compositions.

In Chapter 6, the results from the spectroscopic studies of plant oil boundary films via surface-enhanced Raman spectroscopy (SERS) are presented. The objective of this work was to study the chemical nature and adsorption characteristics of plant oil boundary films, and further investigate the mechanisms of growth in plant oil boundary films.

The final chapter concludes this thesis by summarizing key findings and providing recommendations for future work to be conducted. 


\section{Journal Publications and Conference Presentations Arising from this Work}

\section{Journal Publications}

- Chua, W., Stachowiak, G.W.: The Study Of The Dynamic Thickness Of Organic Boundary Films Under Metallic Sliding Contact. Tribol. Lett. 39, 151-161 (2010)

- Chua, W., Stachowiak, G.W.: The Growth of Thin Lubricating Films of Plant Oils. Tribol. Lett. 41, 451-462 (2011)

- Chua, W., Chapman, P., Stachowiak, G.W.: Surface-Enhanced Raman Spectroscopy of Tribochemically-formed Boundary Films of Refined and Unrefined Canola Oils. J. Am. Oil Chem. Soc. 89(10), 1793-1800 (2012)

\section{Conference Presentations}

- Surface-Enhanced Micro-Raman Spectroscopy of Triboformed Boundary Films of ColdPressed Canola Oil. Oral Presentation at ASIATRIB 2010, Perth, Australia, 5-9 December 2010. 


\section{Statement of Candidate's Contribution}

Chapters 3, $\mathbf{4}$ and $\mathbf{5}$ present articles that have been co-authored. They are as follows:

\section{In Chapter 3 and 4,}

Chua, W. (80\%), Stachowiak, G.W. (20\%): The Study Of The Dynamic Thickness Of Organic Boundary Films Under Metallic Sliding Contact. Tribol. Lett. 39, 151-161 (2010)

\section{In Chapter 5,}

Chua, W. (90\%), Stachowiak, G.W. (10\%): The Growth of Thin Lubricating Films of Plant

Oils. Tribol. Lett. 41, 451-462 (2011)

\section{In Chapter 6,}

Chua, W. (70\%), Chapman, P.(20\%), Stachowiak, G.W.(10\%): Surface-Enhanced Raman Spectroscopy of Tribochemically-formed Boundary Films of Refined and Unrefined Canola Oils. J. Am. Oil Chem. Soc. 89(10), 1793-1800 (2012)

\begin{tabular}{|c|c|c|c|}
\hline & Name & Signature & Date \\
\hline Candidate: & Wenhsi Chua & enten. & 14 June 2013 \\
\hline Supervisor: & Prof Gwidon W. Stachowiak & & $17 / 06 / 2013$ \\
\hline
\end{tabular}




\section{List of Symbols}

$A_{a} \quad$ Apparent area of contact

$A_{n r} \quad$ Apparent area of contact less the real areas of contact

$A_{r} \quad$ Real area of contact. For $A_{r} / A_{a}=1, A_{r}=\pi\left(\frac{3 W R_{b}}{2 E^{\prime}}\right)^{2 / 3}$

$C_{b} \quad$ Capacitive contribution outside the apparent area of contact

$C_{n r} \quad$ Capacitive contribution of $A_{n r}$

$C_{\text {air }} \quad$ Reference capacitance measured in air

$C_{r} \quad$ Capacitive contribution at $A_{r}$

$C_{t} \quad$ Total capacitance $=C_{b}+C_{n r}+C_{r}$

$E_{1}, E_{2} \quad$ Elastic modulus of the rubbing solids

$E^{\prime} \quad \quad$ Reduced elastic modulus where, $\frac{1}{E^{\prime}}=\frac{1}{2}\left(\frac{1-v_{1}^{2}}{E_{1}}+\frac{1-v_{2}^{2}}{E_{2}}\right)$

$n \quad$ Refractive index

$p \quad$ Pressure (in $\mathrm{MPa})$

po Atmospheric pressure, $0.1 \mathrm{MPa}$

$p_{a} \quad$ Mean apparent contact pressure

$r \quad$ Radial coordinates of the contact

$R_{a} \quad$ Centre-line average of the surface roughness

$R_{b} \quad$ Radius of the ball $=4 \mathrm{~mm}$ 


\begin{tabular}{|c|c|}
\hline$R_{b}$ & Radius of the Hertzian contact area \\
\hline $\mathrm{R}_{w}$ & Radius of the ball wear scar \\
\hline$z$ & Axial coordinates of the contact \\
\hline$z_{d}$ & Maximum deflection of the contact (at $r=0), R_{b}-\sqrt{R_{b}^{2}-R_{h}^{2}}$ \\
\hline$z$ & Minimum film thickness of the contact \\
\hline$z_{y}$ & Wear depth of the ball, $R_{b}-\sqrt{R_{b}{ }^{2}-R_{w}{ }^{2}}$ \\
\hline$\varepsilon_{\text {air }}$ & Dielectric constant of air, taken as 1.00059 \\
\hline$\varepsilon_{b}$ & Dielectric constant of the bulk lubricant \\
\hline$\varepsilon_{0}$ & Permittivity of free space \\
\hline$\varepsilon_{r}$ & Dielectric constant of the boundary layer at Hertzian pressure \\
\hline$\varepsilon_{\infty}$ & Dielectric constant at high frequencies, approximated by $n^{2}$ \\
\hline$\eta$ & Dynamic viscosity \\
\hline$x_{S}$ & Mean curvature of the surface profile \\
\hline$\varrho$ & Density at the pressure of interest \\
\hline$\varrho_{0}$ & Density at atmospheric pressure and test temperature \\
\hline$\sigma$ & Standard deviation of surface roughness \\
\hline & Poisson's Ratio of the rubbing solids \\
\hline
\end{tabular}




\section{Introduction}

\subsection{BACKGROUND}

Plant oils and animal fats were used by mankind as lubricants, even before recorded history. Evidence of their use in pre-historic times can be found in an Egyptian tomb that dates back to $1400 \mathrm{BC}$, where beef or mutton fat was discovered on the axles of chariots [1]. However, the course of history changed dramatically on August 27, 1859, when 'Colonel' E. Drake drilled sixty-nine feet below the ground near a small village called Titusville in Pennsylvnia, and struck oil [1]. The over abundance of this 'rock oil' meant that they had to resort to using wine barrels for storage. This is the origin of the 'barrel' unit of measurement. Many of those seeking a quick fortune soon followed Drake's footsteps and applied the same drilling technique to other parts of the world.

At the time, industrial development was centred around coal-fired, steam powered engines. It soon picked up pace at the end of the $19^{\text {th }}$ century with the invention and widespread adoption of internal combustion engine, and soon after, the automobile. The rapid growth of the automobile industry by the turn of the century, created a demand for fuel and lubricants that far exceeded the supply of natural oils [2]. The discovery of 'rock oil', with its abundance and low cost, meant that it was able to meet this demand required by the new technologies. The Industrial Revolution had begun, and oils derived from plants and animals for use as lubricants soon became redundant. 
However, mineral oil-based lubricants have poor biodegradability and can also be extremely toxic. The effects of major oil spills from oil tankers and illegal disposal of used oil resulted in widespread damage on our natural ecosystems. For example, as little as a single drop of mineral oil can pollute one cubic metre of water and the contamination can last for up to a hundred years [3]. Some ecosystems are particularly sensitive to changes in water conditions, such as mangroves, lakes and coral reefs, and could take many years to recover from an oil spill. The impact on natural ecosystems could in turn, affect human health through the consumption of contaminated water and seafood. Economies that depend largely on fisheries could also be significantly affected.

$50 \%$ of all lubricants sold worldwide end up in the environment either by design (e.g. total loss lubricants), illegal dumpings, spillages or leakages [4-8]. In Europe, about 40-50\% of approximately 5 million tonnes of used lubricant end up polluting the environment. Estimates for loss of hydraulic fluids are as high as 70-80\% [8]. In 1999, it was estimated that China consumed 3.15 billion $\mathrm{kg}$ of lubricants, of which, no less than 250 million $\mathrm{kg}$ was released into the environment and this is expected to rise by approximately $20 \%$ by the year 2010 [9]. Total worldwide lubricant demand is expected to be about 41.8 million metric tons or 13 billion gallons according to a 2007 Freedonia report [10]. At present, the fastest growth is in the Asia/Pacific region, with China being the largest contributor to this growth. Based on these estimates, 4-6 billion gallons of lubricant would be lost to the environment every year. Without measures to curb the release of non-biodegradable and toxic lubricants and chemicals into the environment, the world's ecosystems would be under severe threat.

Since the 1980s, there had been increasing concerns over the environmental damage caused by mineral oils. This increased environmental awareness has provided a stimulus for the replacement of mineral-based lubricants with biodegradable and non-toxic alternatives, particularly in total-loss lubricant applications, such as in two-stroke engine oils, chain saw oils 
and metal-cutting fluids [3-6]. In addition, the increasing price of petroleum products, the depleting fossil oil reserves and declining rate of oil field discoveries in recent years, have also urged governments to look into renewable and sustainable alternatives [10]. The risk to national security as a result of an overdependence on foreign oil supplies, coupled with the political volatility of the Middle East, a region which holds the world's largest reserves of crude oil, is also another push factor.

Europe is currently leading the way in the use of environment-friendly alternatives to replace mineral oils in many industrial applications. In 1991, Austria imposed a regulation that all forestry chain saws, which are total loss lubricant applications, have to use biodegradable lubricants [4-7]. In the same year, Portugal imposed a regulation mandating the use of biolubricant two-stroke engine oils in outboard boat engines. In Italy, taxation laws are in place for mineral oil-based products. In Germany, R\&D activities have been receiving governmental financial support contributing to new non-food agricultural products. In 2000 , the German government rolled out a program, called the Market Introduction Program (MIP), to subsidize all additional costs when companies switched from mineral oils to biodegradable lubricants [4]. In the US, a BioPreferred program the result of the Farm Security and Rural Investiments Act of 2002 (FSRIA) to promote the use of biobased products by developing a procurement program that requires that Federal Agencies to consider bio-based products from a preferred product list (compiled by the USDA) when purchasing lubricants [10].

\subsection{BIODEGRADABLE LUBRICANTS}

Biodegradation occurs in the initial stages when oils transform into carboxylated or hydroxylated intermediates, and become more soluble in water [2]. The rate of this transformation is termed as primary biodegradability and is measured by the CEC-L-33-A-93 test. It measures the loss of the C-H stretching vibrations in the IR spectrum over a period of 21 days. The second stage of this process is called ultimate biodegradation and is measured 
according to OECD 301B. The test measures the amount of carbon dioxide evolved from a flask containing the oil over a period of 28 days and this is then compared against the organic carbon content of the sample. A substance is considered readily biodegradable if more than $60 \%$ of the carbon content has been released as carbon dioxide $[2,4,11]$. Vegetable oils generally have an ultimate biodegradability of 75 to $95 \%$, compared to 10 to $40 \%$ for mineral oils.

Biodegradable lubricants can be divided into three main categories [4], namely:

- Naturally occurring oils - vegetable oils, such as soybean oil, sunflower oil and rapeseed oil, and animal fats

- Bio-based synthetic oils - synthetic esters based on chemically modified vegetable oils and transesterified ester oils with natural fatty acids, for example, trimethylolpropane (TMP) esters.

- Fully synthetic oils - Polyalkylene Glycol (PAG) and low viscosity polyalphaolefins (PAO2).

Synthetic biodegradable lubricants, like PAG and PAO2 have exceptional oxidation stability and can last as much as 5 times longer than mineral based oils. However, they may cost 3 to 30 times more [4]. A less costly alternative, compared to synthetic oils, is lubricants derived from plant oils. Neat plant oils themselves, have inherently better tribological properties than mineral base oils, and thus positioned themselves to be a promising and low cost source of material for the manufacture of high performing lubricants. Apart from their ecological benefits of excellent biodegradability and low toxicity, neat plant oils, in fact, perform better than mineral base oils in many ways. They have high flash point, low volatility, low evaporation loss, high viscosity index and much better anti-wear and friction reducing properties [2-4]. Such tribological performances can only be matched by mineral base oils, 
through the blending of additives. The drawbacks, however, are that plant oils suffer from poor thermal stability and tend to oxidize fairly quickly. They also tend to have high pour points, which makes them unsuitable for use in low temperatures $[4,7]$. Current research efforts are thus aimed at improving these properties, and they include:

- Genetic modification at the cultivation stage

- Chemical modification at the post-refining stage

- Additive technology

- Blending with oxidatively more stable lubricants

Much of the research efforts have been focused on increasing the oxidation stability of plant oils and great strides in the performance of bio-based lubricants have been made in the last decade [10]. Genetic modification are aimed at producing plant oil base stocks that inherently have desirable molecular structures, such as saturated and monounsaturated oils which have less double bonds, and are hence less susceptible to oxidation [7]. However, more saturated oils have a higher pour point compared to less saturated oils, due to the susceptibility of the fatty acid chains of the triglyerides to align at low temperatures and thus crystallize. This problem could be remedied by chemical modification, through branching [8]. The cold flow properties and oxidation stability of the oils can be improved by branching, since the double bonds are eliminated. The alignment of the fatty acid chains in the crystallization of fats are impeded by the attachment of other alkyls and radicals at the double bond positions. This was demonstrated in epoxidized vegetable oils and estolide esters derived from vegetable oils [11-12].

Despite these developments, penetration of bio-based lubricants into the lubricant market remains slow. The greatest success achieved by a bio-based lubricant thus far, has been in transformer oils [10]. Development of a soybean-based transformer started in the early 
1990s, after a costly oil spill in Iowa, USA. A bio-based transformer oil, known as Biotrans100 was developed by a joint venture by Waverly Light and Power, Ag-based Industrial Lubricants Research Program (ABIL), ERMCO and Cargill. The bio-based oil has proven to exceed mineral oil-based transformer oils in terms of performance and economics. In addition to biodegradability and low toxicity, it has a fire point which is above $300{ }^{\circ} \mathrm{C}$, which is approximately twice that of a comparative mineral oil based product. Service life of transformers has also been proven to increase substantially due to the increased life of the paper insulation when bio-based transformer oils are used [10,13]. Vegetable oils, being more polar than petroleum oils, have the ability to draw moisture from the paper, reducing the rate at which the insulation breaks down by hydrolysis. Bio-based transformer oils currently hold the largest share of the bio-based lubricant market to date. Demand for bio-based transformer oils in 2008, as reported by Cooper Power Systems, reached approximately 6 million gallons per year, and this is expected to increase to 20 million gallons by the year 2011 [10].

Other promising sectors in the lubricant market for bio-based lubricants include hydraulic oils, cutting fluids, chain saw oils, two-stroke cycle engines, wire rope greases and railroad lubricants $[4,10]$. However, market penetration for bio-based oils in these sectors has thus far been comparatively much poorer than transformer bio-based oils. The main reason for this is that they do not offer significant advantage over mineral oil based products in terms of performance and economics. Unless the performance can be improved and the prices can be lowered, growth of these environmentally friendly alternatives would be dependent on governmental regulation or the use of economic tools, such as taxation and subsidies. This would be further discussed in the following section addressing the sustainability of bio-based lubricants. 


\subsection{SUSTAINABILITY AND GREEN TRIBOLOGY}

The assumption that renewable resources, such as bio-based lubricants are sustainable or 'green', should be thoroughly questioned [2]. This issue has been a subject of considerable debate in the scientific community. Being biodegradable alone, does not necessarily guarantee that a product is a sustainable alternative. The conversion to bio-based lubricants must be taken from a holistic perspective. For instance, a bio-based lubricant could be more detrimental to the environment if it consumes more energy to produce, deprives arable land for the production of food and/or requires a significantly reduced oil change interval, and this would be dependent on the context in which the lubricant is applied. Other factors, such as eco-social impacts, production energy costs and the life cycle of the bio-based lubricant must therefore be taken into account $[2,6,14-16]$. The replacement of mineral oil lubricants with bio-based lubricants in any particular application must therefore, firstly be evaluated via an economic and environmental risk assessment.

One of the key factors in evaluating the need for this conversion is the location and application of the lubricant. For example, the use of mineral oils poses a greater and immediate threat to flora and fauna if it is used in an agricultural or marine environment, as opposed to a factory based in the metropolitan area. Similarly, a lubricant used in a total loss application, such as a chain saw, in an agricultural setting, has greater environmental consequences than a cutting fluid used in a machining workshop. The case for conversion to biodegradable lubricants from mineral based lubricants should therefore, be focused on areas where the likelihood of an intentional or unintentional leak would cause a direct and significant environment impact. These areas include equipment used in agricultural and marine environments, such as chain saws, tractors, marine 2 -stroke cycle engines. The consequential costs of a clean-up and ecosystem recovery due to a spill, and the loss of public trust are great 
motivators for this change. The change in these areas must occur out of necessity and if need be, should be imposed by government legislation and regulations.

In other areas where exposure to the risk of environmental pollution is not as critical, the conversion to plant oil based lubricant should be promoted via governmental tools such as subsidies on plant oil products and/or taxation on mineral oil based products. These noncritical applications include hydraulic fluids and cutting fluids used in industrial areas that are not situated near waterways, lakes and rivers. For cutting fluids, the property of oxidation stability for lubricating oils is not as critical as its ability to lubricate and provide cooling during cutting processes [17]. In these applications, plant oils can be used without the need for extensive chemical modifications or additive blending. Production costs of bio-based lubricants for such applications can thus be kept low and this would in turn, encourage users to convert. This applies similarly to the case of transformers oils used in sealed transformers where atmospheric exposure of the oils is minimal.

The major proportion of the lubricant market, however, belongs to crankcase oils, particularly for automobiles [10]. However, there is little impetus for bio-based lubricants to replace mineral oils in this sector. Bio-based engine oils are generally more expensive than mineral oil-based engine oils due to the chemical modifications required for bio-based oils to meet engine oil API standards. Given the higher price of these lubricants, it is difficult to convince lubricant users to convert from mineral oil based lubricants. Furthermore, motor vehicle owners are less likely to convert to bio-based lubricants, since such conversions would likely void most car manufacturers' warranty. Despite significant improvements made to the oxidation stability of bio-based motor oils, the market perception has not changed.

Part of the motivation for converting to bio-based motor oils is that mineral oils contain phosphorus containing ZDDP additives that poison the catalytic converters [18]. However, the European emissions standards that have in recent years come into force and car 
manufacturers have now taken steps to reduce carbon emissions by moving towards smaller, forced induction engines that are more fuel efficient and produce fewer emissions, for the same or higher level of performance. Mineral oil-based engine oil formulations have consistently been modified to meet these emissions standards (see Table 1.1 below). The high price of petrol and diesel in recent times have also encouraged road users to buy more economical vehicles with smaller engine capacities and lower fuel consumption. Sales of petrol-electric hybrid cars have also increased significantly over the last decade. As a result, exhaust gases from motor vehicles are now significantly less toxic than a decade ago, and hence the drivers for converting to bio-based lubricants have become less convincing.

Table 1.1: Phosphorus and sulphur limits in engine oil specifications [18]

\begin{tabular}{lcl} 
Year & API Specification & Limits \\
\hline 1989 & SG & No P, S limits \\
1994 & SH, GF-1 & $\leq 0.12 \%$ wt, P \\
1997 & SJ, GF-2 & $\leq 0.10 \%$ wt, P \\
2000 & SL, GF-3 & $\leq 0.1 \%$ wt. P \\
2004 & GF-4 & $0.06 \%$ wt $\leq$ P $\leq 0.08 \%$ wt., \\
& & $\leq 0.5 \%$ wt. S (SAE 0W and 5W muiltgrades) \\
& & $\leq 0.7 \%$ wt. S (SAE 15W muiltgrades)
\end{tabular}

A class of bio-based lubricant that shows promise in the automobile market is synthetic esters. Fuchs has already marketed a line of environmentally friendly lubricants, known as the Planto lubricants, which are synthetic esters derived from plant oils. Synthetic esters offer excellent tribological properties and have higher oxidation stability compared to mineral based lubricant formulations. At present, however, plant oil-based lubricants are, in general, still more costly to produce than mineral oil-based lubricants, and are generally priced 
twice as much. Reducing the cost of production would be paramount to the expansion of biobased synthetic esters in the automobile market.

It is the author's opinion that research and development for bio-based motor lubricants should be geared towards producing better performing lubricants that can compete with mineral oils on price, without trying to meet International or European 'biodegradable' or 'non-toxicity' standards. Trying to meet these standards, while improving the oxidation stability would be a futile exercise, since any increase in oxidation stability generally comes at the expense of biodegradability to some degree, regardless of how biodegradability is defined. Diversification of the base oil sources, and not biodegradability, should be the main motivation for developing plant oil-based motor lubricants. Without developing bio-based oils that can compete with mineral oils on price and performance, market penetration and attracting further commercial investments for research would be challenging.

From a broader perspective, an increase in the demand for oil producing crops could generate a stronger growth in agricultural sectors of the economy and create jobs for rural areas. It also reduces the economy's dependence on the import of foreign oil supplies and diversifies the economic risks. On the other hand, another school of thought argues that crops cultivated to produce bio-based products such as fuel and lubricants also compete with food crops for arable land, thus lowering the available food supply and pushing up food prices $[2,6$, 10, 19]. There is already a shortage of available arable land for the cultivation of crops for food given the high growth rate of global population, which currently stands close to 7 billion. The effect is compounded by the fact that many of these crops are grown in Third World countries, and could thus create other economic-social problems. This has been the subject of the greatly discussed 'fuel versus food' debate over recent times.

The problem could be partially remedied by the use of non-food crops, such as algae, for the production of plant-based products. Algae are a renewable source of oil that is carbon 
neutral. However, the technology is at its infant stages. It is however, an interesting alternative to the cultivation of food crops for oil, since their cultivation may be independent of seasonal change and they do not compete with food crops for arable land [20-21]. They can also grow in relatively arid conditions. The algae can be harvested and processed to produce omega-3 oils for pharmaceuticals, biodiesel, and the dried by-product can be used as protein feed for fisheries, so that nothing is left to waste. Aurora Algae Inc. currently owns a demonstration algae plant with 6 one acre ponds in Karratha, Western Australia. Carbon dioxide emitted from surrounding industrial plants can be used to grow the algae and as a result, reduces the net carbon dioxide emissions from these industries.

It has also been argued that the issue of sustainability would be better addressed in the development of lubricant recycling and disposal technologies. Improvements in present mineral oil-based formulations could also further improve the oxidation stability and extend the oil change interval. More robust catalytic converters could also be developed to mitigate the risks of catalytic poisoning by ZDDP additives in lubricant formulations. Perhaps a more long term and effective solution would be the investment on public education and awareness on the dangers of contaminating our rivers and lakes with toxic chemicals such as used lubricants. While the above arguments hold true, it is undeniable that there is an overdependence on the world's depleting fossil oil reserves, and it is inevitable that a diversification on our resources must be made to be sustainable.

\subsection{CONCLUSIONS}

The main challenge facing environment-friendly alternatives is that the overall production costs still remain relatively high compared to mineral oil-based lubricants, which have the advantage of economies-of-scale. Although the price of crude oil has risen significantly over the last two decades, the cost of cultivation, extraction and refining plant oil based lubricants still exceeds the cost of producing mineral oil-based lubricants. The market perception of 
plant oil based lubricants as an inferior product and an unproven technology also poses as major deterrents to their penetration in the lubricant market. The higher price of modified plant oils also discourages lubricant users to convert from mineral based oils to plant oils. Public acceptance of bio-based lubricants must start from areas where a replacement of mineral oil based lubricants with bio-based ones is deemed to be critical for the sustainability of the environment, i.e. in agricultural and marine applications. Government intervention would be critical in accelerating this process.

The key to increasing the market share of bio-based lubricants in the market would be a reduction in the price of production. This can be lowered by technological improvements in the areas of gene modification, cultivation, extraction, refining and additive technology. Research opportunities also exist in improving the tribological performances of bio-based lubricants, including fundamental tribological research, where our knowledge of plant oil boundary lubrication is still limited. A further understanding of their tribological mechanisms would be essential for the formulations of better performing bio-based lubricants. Additive technology, specifically for bio-based lubricants, would also need to be further developed. Ultimately, both the reduction in costs and improvements in performance must be sufficient so that the motivation for lubricant users to covert to bio-based lubricants would be an economical one, and not environmental.

\section{REFERENCES}

1. Yergin, D.: The Prize: The Epic Quest for Oil, Money, and Power. Simon \& Schuster, New York

2. Boyle, S.: Green lubricants. Environmental benefits and impacts of lubrication. Green Chemistry 4, 293-307 (2002) 
3. Goyan, R.L., Melley, R.E., Wissner, P.A., Ong, W.C.: Biodegradable lubricants, Lubri. Engr. 35, 303-308 (1978)

4. Theo, M., Dresel, W. (eds): Lubricants and Lubrication, 2nd Ed. Wiley-VCH, GmbH, Weinheim (2007)

5. Bartz, W.J.: Lubricants and the environment. Tribol. Int. 31, 35-47 (1998)

6. Mann, S.: Ranking without valuing in the face of major uncertainty - The case of the promotion of biodegradable lubricants. J. Enviro. Mgmt. 85, 198-203 (2007)

7. Wagner, H., Luther, R., Mang, T.: Lubricant base fluids based on renewable raw materials. Their raw catalytic manufacture and modification. Appl. Catal. A: General 221, 429-442 (2001)

8. Schneider, M.P.: Plant-oil-based lubricants and hydraulic fluids. J. Sci. Food Agric. 86, 1769$1780(2006)$

9. Wang, B., Tao, D.H.: Rheological and tribological characteristics of the synthesized lubricants derived from vegetable oils. J. Shanghai University 9(5): $462-465$ (2005)

10. Bremmer, B.J., Plonsker, L.: Bio-based lubricants. A market opportunity study update. November 2008. Prepared for the United Soybean Board. (2008).

11. Cermak, S.C., Isbell, T.A.: Synthesis and physical properties of estolide-based functional fluids. Ind. Crops Prod. 18, 183-196 (2003)

12. Wu, X., Zhang, X., Yang, S., Chen, H., Wang, D.: The study of epoxidized rapeseed oil used as a potential biodegradable lubricant. J. Am. Oil Chem. Soc. 77, 561-563 (2000).

13. Fields, S.: One slick trick. Building a better biolubricant. Enviro. Health Persp. 111(12), 654657 (2003) 
14. Herrmann, C., Hesselbach, J., Bock, R., Zein, A., Öhlschläger, G., Dettmer, T.: Ecologically benign lubricants - Evaluation from a life cycle perspective. Clean 35(5), 427$432(2007)$

15. Cunningham, B., Battersby, N., Wehrmeyer, W., Fothergill, C.: A sustainability assessment of a biolubricant. J. Ind. Ecol. 7, 179-192 (2004)

16. Miller, S.A.., Landis, A.E., Theis, T.L., Reich, R.A.: A comparative life cycle assessment of petroleum and soybean-based lubricants. Enviro. Sci. Technol. 41, 4143-4149 (2007)

17. Shashidhara, Y.M., Jayaram, S.R.: Vegetable oils as a potential cutting fluid - An evolution. Tribol. Int. 43, 1073-1081 (2010)

18. Spikes, H.: The history and mechanisms of ZDDP. Tribol. Lett. 17, 469-489 (2004)

19. Cassman, K.G., Liska, A. J.: Food and fuel for all: realistic or foolish? Biofuels, Bioprod., Bioref. 1, 18-23 (2007)

20. E.W. Becker. Microalgae: Biotechnology and Microbiology. Cambridge University Press, New York (1994)

21. Sheehan, J. Dunahay, T., Benemann, J., Roessler, P.: A look back at the U.S. Department of Energy's Aquatic Species Program: Biodiesel from Algae. Close out report. NREL/TP-580-24190. National Renewable Energy Laboratory (1998) 


\section{Literature Review}

\subsection{INTRODUCTION}

Vegetable oils are essentially composed of triglycerides or esters of glycerine (alcohol or carboxyl group) and long-chain fatty acids (see Fig. 1.1). The fatty acid composition is variable depending on the type of vegetable oil. The fatty acids differ in length and the number of unsaturation sites or double bond sites. These double bond sites are responsible for the poor oxidative stability of the oils [1-2]. In addition, the hydrogen at the $\beta$-position of the carboxyl group is extremely susceptible to hydrolysis, which causes the breakdown of the triglyceride into their individual fatty acid and glycerine components. In areas where high rates of metalto-metal sliding contact are prevalent, exposure to these high temperatures is unavoidable, and this leads to accelerated oxidation and ultimately a breakdown of the lubricant film. The thermal, oxidation and hydrolytic stability can be improved by chemical means which include hydrogenation and epoxidation of the double bond sites of the fatty acids, and the transesterification of the carboxyl group (glycerine) to form more thermally stable esters such as neopentyl glycols and trimethylolpropane [2]. 


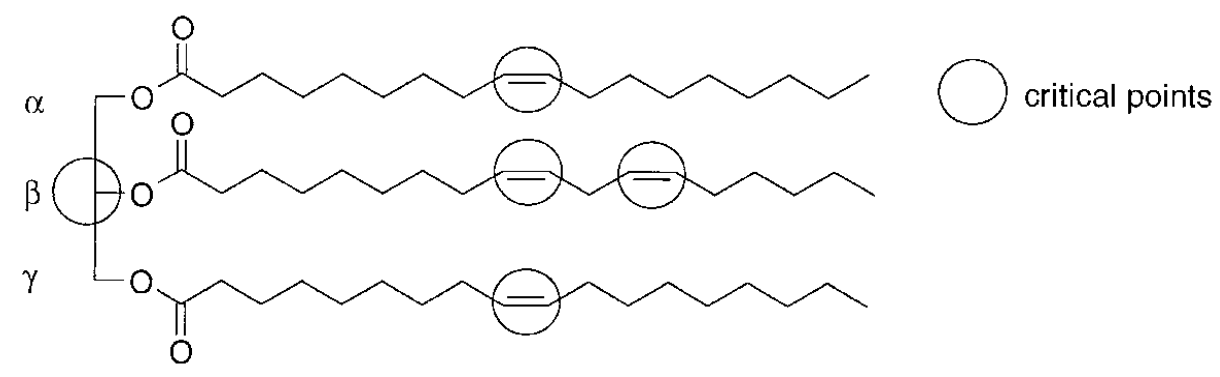

Fig. 1.1: Triglyceride ester of different fatty acids; critical points $-\beta$-CH-group and unsaturated fatty acid double bond sites [2].

\subsection{EFFECT OF MOLECULAR STRUCTURE}

The ability of plant oils to provide good boundary lubrication can be attributed to the fact that the carboxyl group of the triglyceride is relatively polar and will adhere to metallic surfaces, while their fatty acid chains act as brushes. The boundary lubrication mechanism is similar to that of fatty acids as studied by Bowden and Tabor [3]. When assembled onto a surface, the triglycerides form a closely packed monolayer film capable of reducing metallic adhesion during asperity contact and hence reducing friction and wear. Many studies have confirmed that triglyceride esters with higher polarity, lower level of unsaturation and containing fatty acids with longer chain lengths exhibit better friction reducing and wear protection properties [4-5]. The longer chain lengths produce a thicker monolayer on the surface and maintain a larger separation between opposing asperities during sliding which reduces the amount of adhesion. The higher polarity accounts for the stronger adsorption of these molecules to the surface and affects the durability of these films. And finally, the lower level of unsaturation decreases the number of bends in the long chain fatty acid, resulting in a densely packed monolayer film. While this seems to be a very simple model for the boundary lubrication 
mechanism of bio-based lubricants, later experiments conducted reveal that the mechanisms at work are far more complex.

Zhang et al. [6] found that the frictional and anti-wear behaviour of arachidic acid (C20) were better than that of stearic acid (C18) and behenic acid (C22). It is postulated that with longer chains, the molecules tend to be more flexible, which causes its structure to change and the film to break down more easily. With shorter chains, the molecules are stiffer and this disorientation is comparatively reduced. On the other hand, the thickness of the film is compromised. Zhang et. al. postiulated that the optimum boundary lubricating behaviour is thus reached when a balance of stiffness and toughness is reached, which is the case for arachidic acids in this comparison.

Branching or branch groups are sometimes introduced to unsaturated fatty acids at the double bond sites during chemical modification to improve oxidation stability and pour point characteristics $[2,4]$. Branched groups also serve as additional functional groups that increase the polarity and hence the adsorption strength of the molecule. Branching can be achieved by a process known as cooligomerization (see Fig. 1.2). Pooley and Tabor [7] have argued that side groups may hinder translational motion and result in increased friction, whereas Cho and Granick [7] have showed that branched compounds retain better fluid-like performance at higher pressures as compared to linear unbranched chains. On the contary, it is interesting to note that Bhuyan et al [5] observed that, the reverse phenomenon occurs with branched fatty acid chains, i.e. shorter chains actually have a lower friction than those of longer chains (see Fig. 1.3). This suggests that there may exist a combinational effect of base chain length and branching on the boundary lubrication properties. 


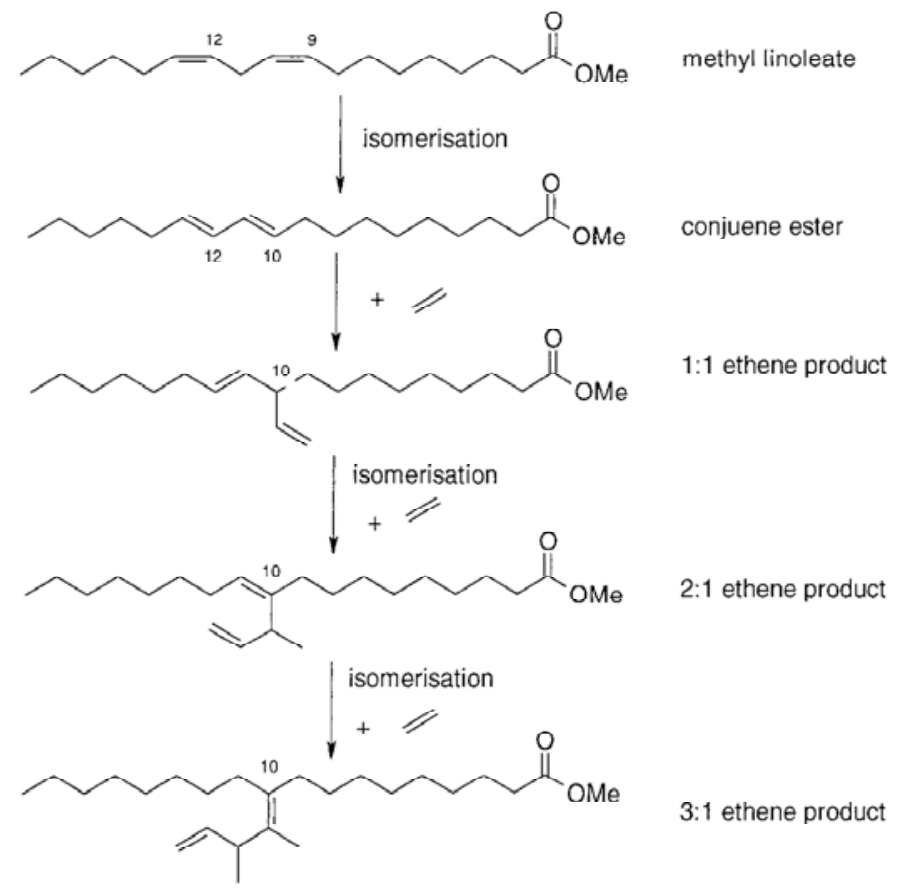

Fig. 1.2: Reaction of methyl linoleate with ethene over $\mathrm{RhCl}_{3} \cdot 3 \mathrm{H}_{2} \mathrm{O}$ [2].

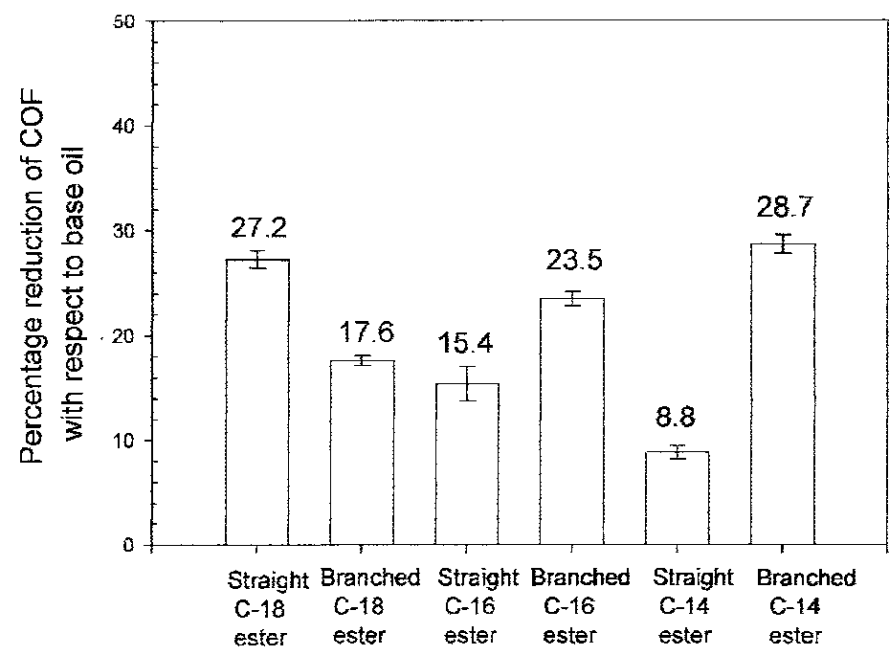

Fig. 1.3: Percentage reduction in coefficient of friction (COF) with respect to paraffin oil as base oil $0.5 \%(\mathrm{w} / \mathrm{w})$ concentration of $\mathrm{C} 18,0.63 \%(\mathrm{w} / \mathrm{w})$ of $\mathrm{C} 16$ and $0.15 \%(\mathrm{w} / \mathrm{w})$ of $\mathrm{C} 14$ of straight and branched chain methyl esters [5]. 
An interesting argument was presented by S. Hsu [8] regarding the trade-off between the adhesive strength and self-reparability of the lubricant. His argument was that while strong films are formed with highly polar molecules, the self-reparability is compromised because these molecules are not free to move about to 'reorganize and self assembled' at the areas which have been mechanically disrupted by the contacts. He suggested four possible molecular layer structure designs that will exhibit a balance of adhesive strength and selfreparability. One of which includes, a combination of long chains and short chains closely packed and adsorbed on the surface. The short chains are stiffer and act as a solid lubricant layer while the longer chains are more spaciously separated and highly mobile, forming a viscous layer over the shorter chains.

Generally, the linear log-log relationship of film thickness and velocity for elastohydrodynamic lubrication (EHL) theory holds up to the point where film thickness approaches the dimensions of the adsorbed or tribochemically formed boundary layer. However, recent studies have shown that this may not be entirely true for all lubricants. For instance, Spikes [10] observed that with stearic acid in hexadecane solution, film thickness was initially in the order of $2 \mathrm{~nm}$ at low speeds, when the test was conducted at increasing speeds. However, on reducing the speeds, the boundary film thickness was found to remain stable at approximately $10 \mathrm{~nm}$. When the motion was halted, the thick film was lost and the thickness was reduced to 1-2 nm. Similarly with vegetable oils, Biresaw [11-12] found that at entraining speeds below $0.1 \mathrm{~m} / \mathrm{s}$, and at EHL film thicknesses less than $50 \mathrm{~nm}$, the pressure viscosity coefficient of the oils is comparatively higher (see Fig. 1.4). Following the ideas of Luo et al. [13], there may exist not only an immobile adsorbed layer on the surface but the presence of a transition phase above that of the adsorbed layer which is highly viscous. The transition between EHL and boundary lubrication is sometimes referred to as the intermediate lubrication or thin film lubrication. This lubrication regime is an interesting field of study, 
since the dynamics of these films is governed, not only by the bulk physical laws of viscous flow and contact pressure, but also by surface forces and intermolecular interaction.

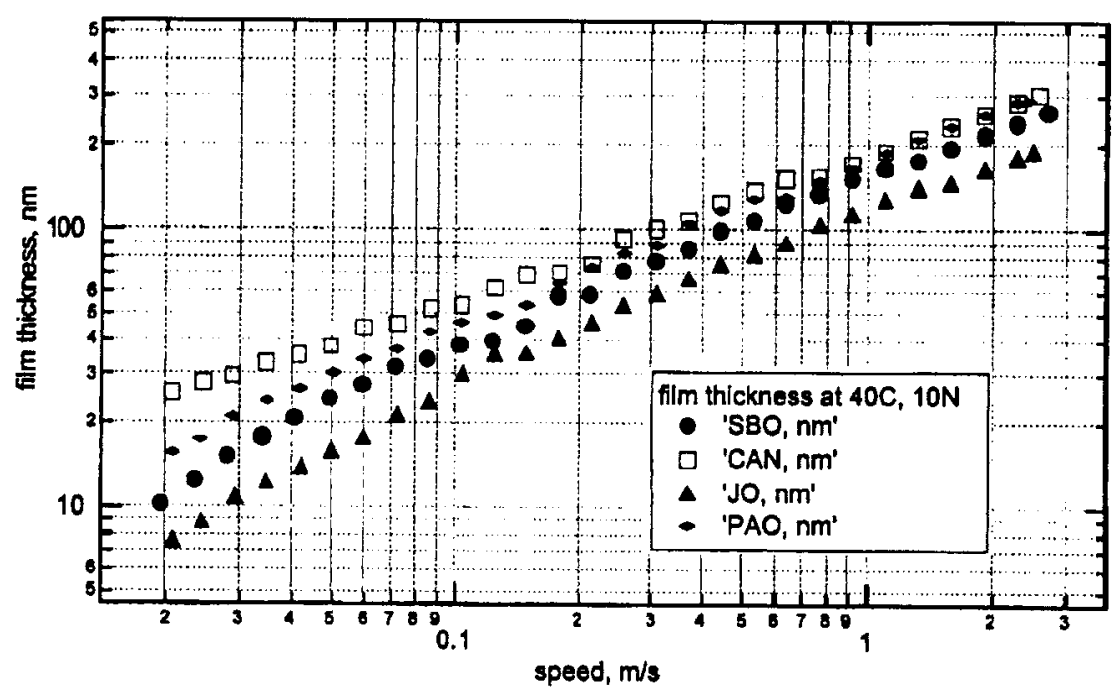

Fig. 1.4: Film thicknesses versus speed curves for soybean oil (SBO), canola oil (CAN), jojoba oil (JO) and PAO [11].

The preceding discussions suggests that while chain length, degree of saturation and polarity are strong indicators of the vegetable oils' performance, they are by no means the critical factors that determine their behaviour. Instead, the stiffness and adsorption strength of each individual molecule, combined with the packing density and lateral cohesion of the molecules should be the governing variables that define their tribological performances. These variables are inherently dependent on the makeup of the molecules' structure.

\subsection{EFFECT OF TRIBOCHEMICAL REACTIONS}

While many studies have been conducted on the tribological properties of plant oils at their initial state of adsorption or chemical reaction with the surface, much is still unknown about the tribochemical reactions that occur during the course of rubbing and the effects this has on the boundary film thickness and friction. Since the rubbing process essentially supplies both 
mechanical and thermal energy repeatedly over the same surface, the nature of these films must therefore be a dynamic one. Within the limits of their shear and adhesion strength, these films formed will either remain stable or grow in thickness. Outside those limits, they will be gradually desorbed by repeated sliding, or in the worse case, be violently sheared away, accelerating wear. Effective films are formed when the rate of adsorption is greater than or equal to the rate of desorption [9], but these rates may be also dependent on contact conditions, such as temperature, load, speed, material properties, and surface topography.

Under the high pressures of a sliding contact, plant oil films may undergo a rapid transition from a liquid-like to a glassy or amorphous state, where the molecules are still in the disordered state but are frozen as a result of the rapid compression [14]. Given the propensity of fatty acid chains to align in the direction of shear, it would be reasonable to suggest that they could possibly adopt more solid-like or crystalline forms. Whether such rapid thermodynamic compressions and shear could have an influence on the tribochemical reactions between the adsorbed lipid molecules and the rubbing surfaces, and between the molecules themselves, is largely unknown. If the molecular structure of the film is altered as a result of tribochemical reactions, the film forming ability and friction would also likely be affected. These changes are of critical importance in practice, since machines are generally lubricated over long periods of time.

Examples of such behaviours could be found in the works of Murakami et al [15]. When Murakami et al. compared stearic (C18), oleic (C18-1), linoleic (C18-2) and linolenic acids (C18-3) doped in paraffinic oil in a four-ball wear tester, they found that polyunsaturated fatty acids have higher scuffing loads and showed better frictional stability at high temperatures. It was postulated that this is due to the formation of friction polymers. In yet another study, Sahoo et al. [16] recently reported that the coefficient of friction (COF) for nhexadecane with linoleic acid (LA) (5\% v/v) was found to decrease over sliding time from 
0.09 to roughly 0.04 , while that of stearic acid (SA) remained relatively unchanged (see Fig. 1.5).

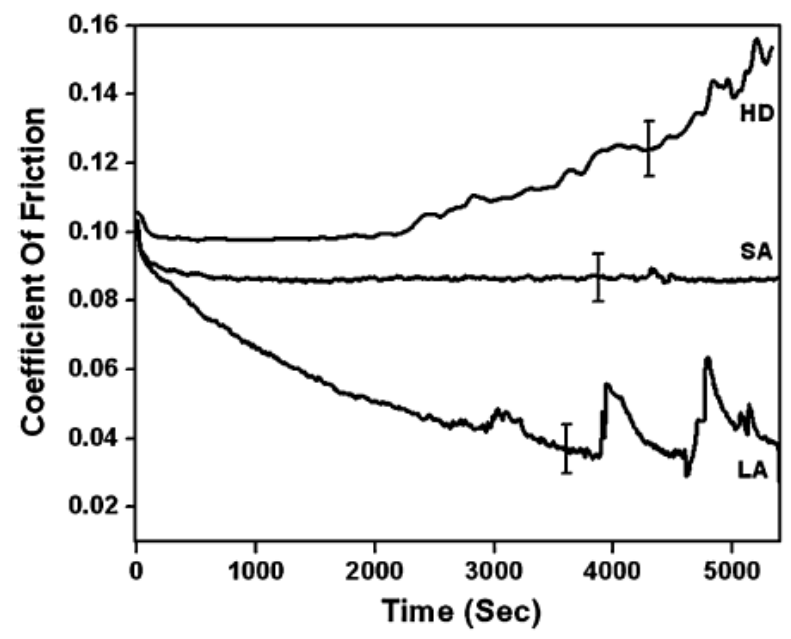

Fig. 1.5: Coefficient of friction of steel against steel pin recorded on pin-on-disc tribometer, lubricated: hexadecane and SA and LA additives $(5 \% \mathrm{v} / \mathrm{v})$ dispersed in $\mathrm{n}$-hexadecane, as a function of sliding time and velocity: normal load $=60 \mathrm{~N}$ (mean contact pressure $p_{\mathrm{m}}=10 \mathrm{MPa}$ ), sliding velocity $\sim 0.35 \mathrm{~m} / \mathrm{s}$. [16].

The double bonds of polyunsaturated plant oils have been widely known to be responsible for the oils' susceptibility to oxidation [1-2]. Oxidation of plant oils generates other compounds that will likely influence the tribological properties. In an investigation by Fox et. al. [17] with sunflower oil, an initial increase in friction and wear was observed when the oil was kept for the first 22 days, followed by a significant drop in friction and slight decrease in wear after 28 days of accelerated oxidation. Fox et. al. also observed this change in friction when testing sunflower oil doped with free fatty acids, where the friction varied with sliding time depending on degree of unsaturation, concentration of the free fatty acids and temperature [18]. Free fatty acids are known to accelerate the oxidation processing of vegetable oils. In the advance stages of oxidation, polymerization processes involving chainbranching and cross-linking mechanisms may occur. 
It is uncertain whether the tribological improvements observed by Fox et. al. was due to the increased level of free fatty acids due to oxidation, or the formation of polymeric films. It was suggested that, for the case of the severely oxidized oil, secondary oxidation compounds, such as ketones and epoxies, might have acted as precursors for which polymeric films are formed. The generation of oxidation and polymerization products could be accelerated at the sliding interface, particularly at the asperities, where high pressures, temperatures and shear forces are present. This would alter the tribological characteristics of the boundary film formed. The formation of polymeric films is termed as tribopolymerization.

\subsection{TRIBOPOLYMERIZATION}

The use of tribopolymerization as a mechanism to produce friction and wear reducing films on surfaces was realized by Michael Furey, a professor at Virginia Technology Institute, USA, more than 30 years ago. He showed that when hexdecane doped with 1\% C36 dimers was able to reduce wear on metallic surfaces as much as $80 \%$ and this is comparable to the performance of the much widely used ZDDP anti-wear additive used in all gear and motor oils [19]. The concept of tribopolymerization is that when surfaces rub against each other, the microscopic points of contact which are exposed to the most extreme of conditions of shear, high temperature and pressure, would cause certain compounds or additives in the oil to activate, chemically react and polymerize. The result is a protective film that is formed at particularly areas of the contact areas that are needed, i. e. at the asperities [20].

Anti-wear additives, which contain phosphorus and sulphur, such as ZDDP, causes exhaust catalyst poisoning in automobiles, which in turn, causes significant amount of toxic gases to be emitted into the environment. The use of tribopolymerization could potentially negate the use of these toxic additives and allow environmentally friendly and ashless lubricant formulations to be developed [21-22]. Better tribological performances can also be achieved by using this technique for the lubrication of non-metallic machine components, such as 
ceramic, since it does not rely on the tribochemical reaction with a metallic surface to create anti-wear films.

The benefits of tribopolymerization has also been validated by Zheng et al [23], who showed that wear reductions of $20-30 \%$ can be ached with 1 wt $\%$ nbutyl-acrylate in hexadecadane. Svahn and Csillag [24] who were working on inorganic fullerene-like (IF) solid particles based on $\mathrm{MoS}_{2}$ or $\mathrm{WS}_{2}$ nanoparticles, recently showed the vast improvements achieved by blending IF particles and e-caprolactumm in hexadecadane, the latter additive being a cyclic amine monomer normally used for producing nylon-6. It is postulated that the monomer adsorbed onto the metallic surfaces and tribopolymerized upon rubbing, effectively trapping these IF particles in their molecular structure. This increased the load carrying capacity of the tribohemically formed films and reduced the friction by preventing the asperities of the opposing surfaces from contact.

Plant oils could also have the capability to polymerize via addition or condensation polymerization. The polymerization of plant oils have been utilized in the manufacture drying oils, coatings, resins, adhesives, hardeners and in paint formulations. They have also been used in the manufacture of biodegradable plastics including polyesters, polyamides and polyurethanes [25-28]. This is possible because the double bonds in the fatty acid chains of unsaturated plant oils are especially reactive and readily react with oxygen, peroxides and other free radicals. The double bonds provide unsaturated plant oils the natural chemical functionality for modifications including branching and crosslinking mechanisms. They can also be cleaved in a process called ozonolysis which splits the fatty acid chain at the double bond into mono- and dicarboxylic acids.

The presence of heat and a metal catalyst are required in almost all polymerization processes of plant oils. Such conditions could be replicated in tribological contact areas for plant oil lubricated surfaces. While there has been a substantial amount of research in the field 
of polymers made from triglyceides, few have worked on the possibility that such polymerization processes could be affected at the interface between rubbing surfaces, and possibly form products that could possibly reduce friction and wear. Tribopolymerization could possibly explain the tribological behaviour observed by Murakami et. al and Sahoo et. al. on polyunsaturated fatty acids as discussed earlier. If so, there is strong possibility that boundary film thicknesses do not remain the same, since polymers have higher molecular weight and are extended structures of the triglyceride molecule. An observation of change in shear stress or friction accompanied by a change in film thickness would strongly suggest the effects of tribopolymerization in plant oil lubricated sliding contacts. However, the simultaneous measurement of film thickness and friction in sliding contacts are rarely conducted, and this is partially due to the limitations of present conventional tribological instruments and film thickness measurement techniques. This shall be further discussed in the following sections.

\subsection{LIMITATIONS OF CONVENTIONAL BOUNDARY LUBRICATION STUDY METHODS}

The study of boundary films has been traditionally conducted using the pin-on-disk tribometer, reciprocating tribometer and four-ball wear tester. These methods have been focused on evaluating the performance of these lubricants under extreme conditions. Very little work has been focused on observing the changes in friction and wear under realistic conditions and under extended periods of time. Part of the reason is that extended tribological tests can take many hours and many cycles for a single test. For example, pin-on-disk tests usually employ the use of a hard sliding ball or pin against a metal disc made of a soft carbon steel such as 1045, and at load conditions where contact pressures that far exceed the yield strength of the softer metal. This allows the tribological tests to be conducted over a shorter period of time. Under such contact pressures, the asperities of the softer metal would deform, 
yield and most likely break up as large particles into the bulk oil surrounding the areas of contact. Such wear particles could interfere with the tribochemical reactions that are occurring at the interface. Moreover, films formed by tribochemcial reaction or polymerization do not have time to grow or develop before they are destroyed by the bulk fracture of the asperities onto which these films have adsorbed.

Another limitation of these tribometers is that neither the frictional traces nor the wear scar measurements reveal much about the thicknesses of the boundary films generated during the course of rubbing. It is thus difficult to make a logical deduction whether frictional changes, if any, are attributable to a change in the amount of asperity to asperity contact, or the change in the viscosity or shear strength of the boundary film itself. To gain a clearer understanding of the nature of these films, it is imperative that experiments be conducted under more realistic conditions, and that the friction and boundary film thickness be measured simultaneously in situ. While friction measurements can be achieved easily with a strain gage setup and has been utilized in all friction measuring tribometers, the measurement of microscopic film thicknesses is more complicated. Several methods exist for the determination of the film thickness, including capacitance methods, optical interferometry methods and atomic force microscopy methods.

\subsection{FILM THICKNESS MEASUREMENT TECHNIQUES AND THEIR LIMITATIONS}

This section of the literature review is dedicated to the brief discussion of different methods of film thickness measurement techniques, particularly for ultra-thin lubricating films including that of boundary films. In the process of selecting a film thickness measurement technique to be used for this work, the pros and cons of different methods are discussed and compared. 


\subsubsection{Optical Interferometry Method}

By far, the method that has been most widely used for film thickness measurement has been the optical interferometry method. Since the invention of the optical interferometry method by Gohar and Cameron [29], it has become an essential tool in tribological and lubricant rheological studies, particularly in the study of EHL. The method utilizes the fact that white light of the visible spectrum is made up of different wavelengths of light from violet to red. The measurement of film thickness using optical interferometry essentially employs this fundamental law of physics.

The method is described as follows. A polished steel ball is loaded against a flat side of rotating glass disc. The side of the disc which is loaded against the steel ball is coated with a very thin chromium layer approximately $30 \mathrm{~nm}$ thick. The chromium layer is semi-reflective and allows part of the white light to pass through. In the presence of a thin lubricant layer between the glass disc and the steel ball, the transmitted portion of the light would pass through the lubricant layer and be reflected off the shiny surface of the steel ball. The other part of the light would be reflected from the chromium layer back to the observer's eye. Due to the different distances that the two beams of light have to travel, destructive or constructive interference will occur when the two coherent beams superimpose on each other. The result is that for a specific thickness of the lubricant film, a specific wavelength/colour of beam will be seen by the observer. This method allows the film thickness profile of an EHL contact to be observed. The method, in fact, was instrumental in providing the concrete evidence needed to prove the original Ertel-Grubin's EHL theory and the characteristic shape of the EHL film thickness profile.

The problem with the original method was that the minimum film thickness that can be measured is approximately $45-75 \mathrm{~nm}$ [30]. This is because the transmitted beam through chromium layer must travel a minimum distance longer than the reflected beam for any 
interference to occur. This cannot be achieved by thickening the chromium layer, since it would lose its semi-reflectivity. The problem was first remedied by Westlake and Cameron [31] by the addition of a transparent silica layer approximately $500 \mathrm{~nm}$ thick between the chromium layer and the lubricant film, which essentially ensures interference occurs even at zero film thickness, i.e. when there is no lubricant film present. The method was further developed by a few other researchers, most notably by Johnston et al. [32] and Hartl et al. [33], with the use of a spectrometer, a CCD camera and computer-assisted image processing for a more precise identification of the colours, the sensitivity of the instrument increased to below $1 \mathrm{~nm}$.

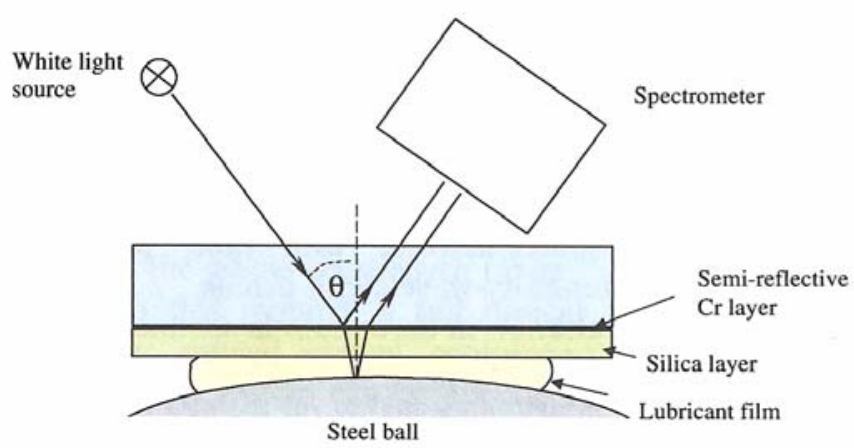

Figure 1.6: Schematic diagram of contact showing two beam reflection and silica layer used in ultrathin film interferometry [34].

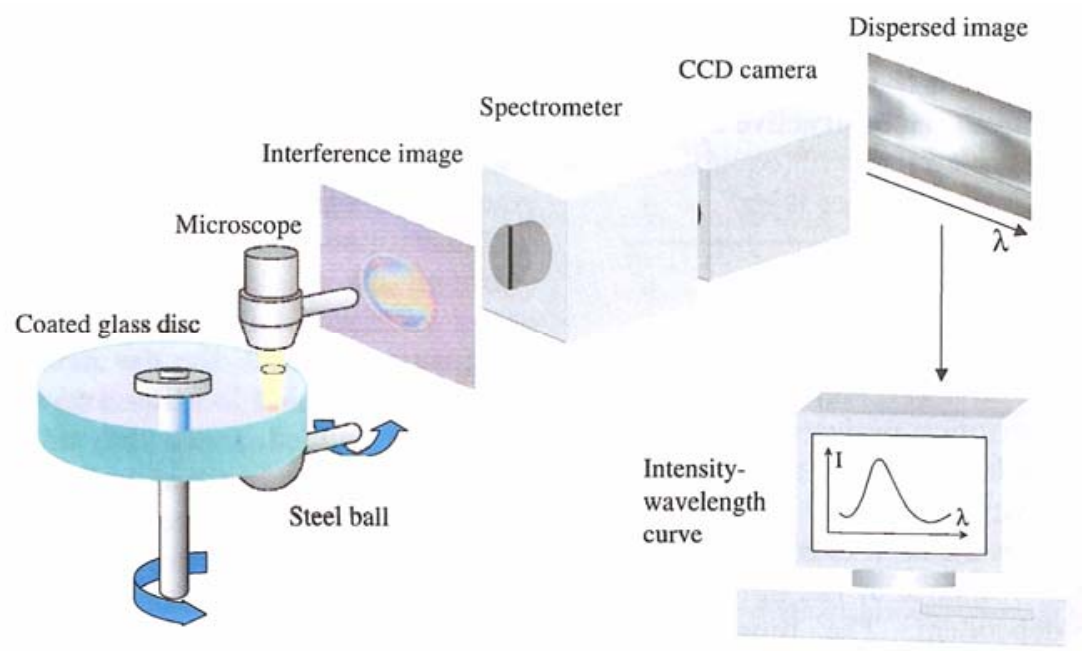

Fig. 1.7: Schematic diagram of ultra-thin film inteferometry set-up. [34]. 
With these groundbreaking developments, the optical inteferometry method, which was initially intended for the measurement of EHL film thicknesses, could now be extended to ultra-thin films in the boundary lubrication regime. The measurement of boundary film thickness, however, poses another challenge to the optical inteferometry method. As noted by the originators themselves, the study of boundary films using optical method is limited by the fact that the thin chromium layer, responsible for the interferometry effect to work, is easily worn off during sliding [35]. As such, the method is limited to the study of boundary films at the initial state of their adsorption to the surface. More so is the lesser stated fact that the thickness of a tribochemically formed boundary film between metallic surfaces depends on the contact conditions during the running-in process, and the effect of these conditions can only be reproduced in an actual metal-to-metal rubbing experiment. During sliding, asperities of opposing surfaces contact and rub against each other. Plastic deformation and, depending on the effectiveness of the boundary layer, welding of these junctions would occur, followed by subsequent fracture of these asperities to expose fresh surface spots. Metallic surfaces emit electrons when new surfaces are formed as a result of this wearing process. Triboemitted electrons interact with lubricant compounds to form radicals and anions that in turn react with the positively charged metallic spots, forming films that protect the surface from further wear. A good example of this can be found in the case of ZDDP films, which relies heavily on the initial running-in period to generate thick films. Such tribochemical reactions play an important role in the organometallic chemistry of lubricated metallic components and the optical method would not be able to replicate such conditions since the method inherently requires one of the surfaces to be transparent, and hence, non-metal.

\subsubsection{Nanotribological Methods}

Advances in nanotechnology have brought about the advent of nano-tribological techniques, such as atomic force microscopy (AFM) or surface force microscopy (SFM). It was a 
modification made to the scanning tunnelling microscope (STM) developed by Binnig and Quate for which they were awarded the Nobel Prize in Physics in 1986 [36-37]. The AFM utlizes a sharp tip, usually made of diamond, which is cantilevered over a sample surface. They calculated that the interatomic spring constants are in the order of $10 \mathrm{~N} / \mathrm{m}$. In comparison, a cantilever spring made of a soft material could be made with spring constants in the range of 0.1-1 N/m [36]. This means that the sharp tip can be made to contact the sample surface without displacing atoms. The concept is similar to that of a surface profilometer but with spring constants and tip radii significantly much smaller. The surface atomic profile is then analysed by measuring the deflection on the cantilever beam using the STM probe or an optical laser device, as shown in Fig 1.8.

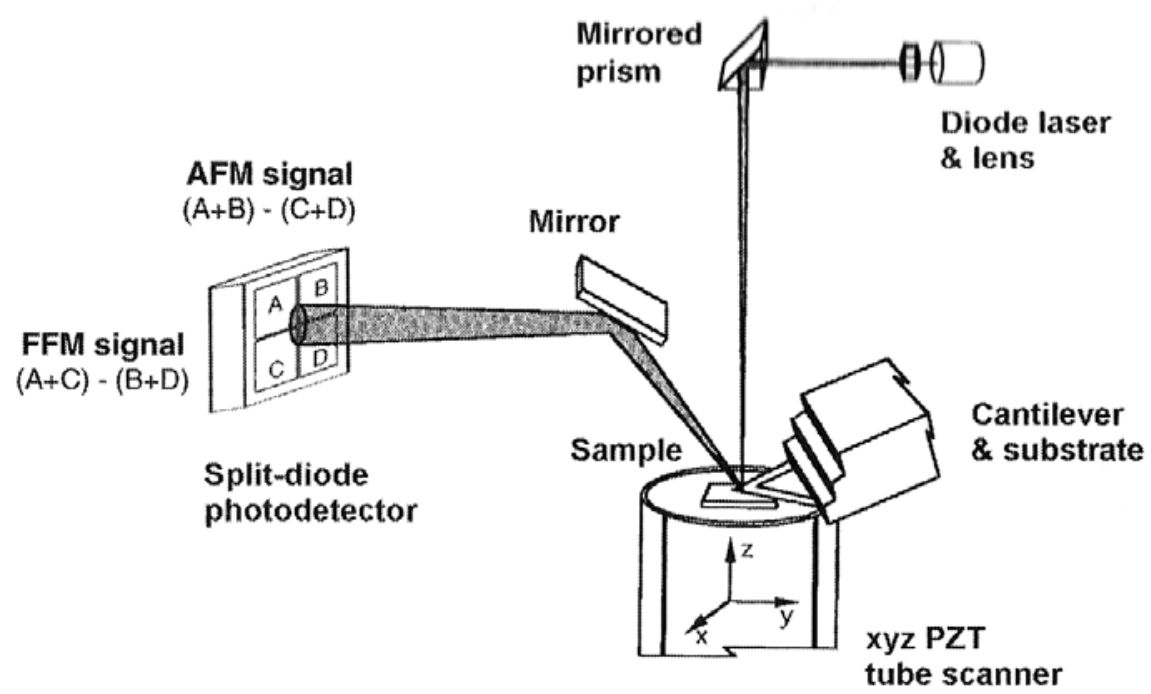

Fig. 1.8: Principle of operation of a commercial atomic force/friction force microscope, sample mounted on a piezoelectric tube scanner is scanned against a sharp tip and the cantilever deflection is measured using a leaser beam deflection technique [38].

This allows a surface profile to be mapped at the atomic level. Frictional forces ca also be measured by increasing the stiffness of the cantilever and the contact forces of the tip. With contact pressures in the order of gigapascals achievable by using a tip radius of 
nanometer dimensions, it is possible to simulate the tribological effect of a single asperity on a sample surface [38]. Since the sharp tip essentially transverse on the top of the film, the AFM method is not specifically designed to provide film thickness measurement. Theoretically, it would be possible, if the surface profile of the film's substrate is known and ideally, atomically smooth, for example, in cleaved mica surfaces. This provides a reference from which the film thickness can be measured against. Both vertical and horizontal deflections of the cantilever beam would then provide film thickness and frictional measurements simultaneously. The AFM method could also be applied to study organic films or biofilms, so long as the cantilever, tip and sample are immersed in a suitable liquid, for example water or ethanol [36, 39]. This is to avoid undesirable effects caused by meniscus forces. The method thus allows the measurement of bio-films in relevant environments, such as the polymerization of fibrin, a protein essential for blood clotting [40].

Though the AFM method provides unprecedented accuracy and precision in the measurement of frictional forces at the atomic level, it does not replicate the actual shearing conditions in real macro-contacts, where flash temperatures at asperity-to-asperity junctions are significantly much higher. In addition, the process of material removal and the presence of entrained wear particles, which may have an influence on the formation of tribochemically reacted films, are not present. As it has been pointed out by Bhusan [38], contact stresses in AFM/LFM studies do not exceed the hardness of the material, which is comparatively much harder at the microscale. As a result, there is usually very little wear. It is worthwhile to mention that in macro-contact rubbing, each asperity will be rubbed the number of times roughly equivalent to the number of asperities across the contact, between intervals in the order of milli or microseconds. If the process of rubbing raises the temperature at these junctions, it follows that each asperity will have very little time to cool before it meets the next asperity. Consequently, flash temperatures would be much higher than those experienced in 
microscale friction measurements. Although AFM/SFM technique provide an interesting perspective of the shear strength of boundary films at the nano-level, its use as a predictor of their macro-tribological properties is somewhat questionable.

Another nanotribological instrument is the surface force apparatus (SFA) first developed by Israelachvili and coworkers [41-42]. The instrument was designed to study the interaction of liquid films between two atomically smooth surfaces, and is capable of measuring forces in the order of $10 \mathrm{nN}$ and film thicknesses less than $1 \AA$ [43] (see Fig 1.9 below).

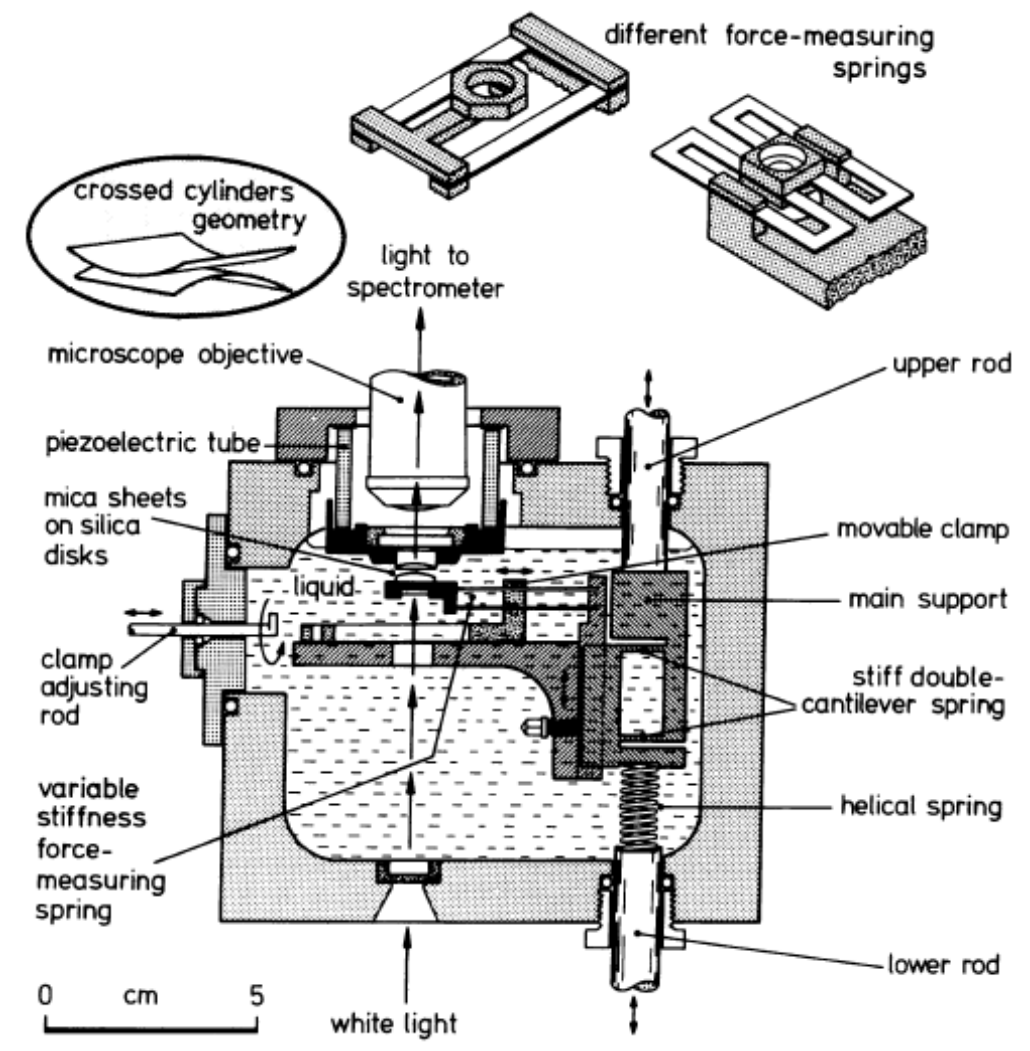

Fig. 1.9: Experimental setup of the surface force apparatus (SFA) Mk II [43].

The gap distance between the two mica surfaces is controlled by a three stage mechanism using micrometers and cantilevers. A fourth stage comprising of a piezoelectric tube, controls the vertical movement of the top mica sample to a precision of angstroms. 
Transverse motion to simulate shearing of films is achieved by the use of a motor-driven micrometer [44]. Frictional forces are measured by the use of strain gauges mounted onto cantilever arms supporting the sample holder. Film thickness measurements are made using the optical interferometry method similar to that described in Section 2.6.1. Since it employs the optical method, it inherits the same limitations where metallic surfaces cannot be used as the substrates. The SFA is extremely accurate and sensitive down to the nanometer scale, and is useful in the study of van der waal and atomic forces between liquid films. However, as with the AFM technique, it does not replicate the tribological conditions experienced in a macrotribological contact. As such, the tribochemical effects of long term sliding on lubricant films cannot be studied.

\subsubsection{Capacitance Method}

The sliding contact should be viewed upon as a complex dynamic tribological system that evolves over rubbing time, where interaction occurs not only between lubricant films adsorbed onto opposing surfaces, but also between the surface and the lubricant film on which it is adsorbed. The representative study of the evolution and dynamic growth of these boundary films in practice therefore lies in their thickness measurement in a metallic macrotribological contact. Therefore, the use of the optical method to measure film thickness will not suffice for the purpose of this research. There is, however, one method which allows this the capacitance method.

Studies of film thickness measurement between metallic contacts can be found in the pioneering works dating back to 1958 by Crook [45], and then later by Dyson et al. [46] in 1965, using capacitance methods. The method works on the principle that two electrically conductive surfaces separated by a dielectric lubricant film essentially form a capacitor. The capacitance of which is determined by the gap between the two surfaces filled by the lubricant, the surface area and the permittivity of the lubricant. However, the works of Crook and 
Dyson et al. were directed towards the measurement of thickness of EHL, rather than boundary films. By using a two roller setup with a thin chromium coating on one of the circumferential surfaces of one of the rollers, Crook was able to experimentally validate the EHL theory and the shape of an EHL contact (see Fig. 1.10 below).

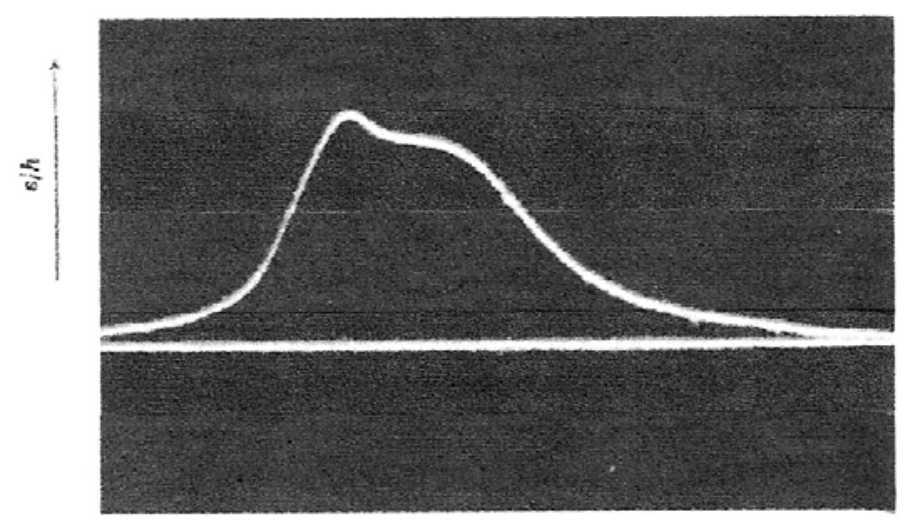

Fig: 1.10: Oscilloscope trace of potential across R [45]

At the time, capacitance measurements were made by manually balancing a capacitance bridge and the task was made more difficult by the frequent short circuiting of the contacting asperities or wear particles being entrained in between the contacting surfaces. Highly precise (down to femtoFarads) capacitance measurements can now be made easily today with commercially available LCR meters with automatic balancing bridges. However, stable readings of contact impedance would still require short circuiting of the contacts to be avoided. The problem is less when a higher excitation frequency is used, but excessively high frequencies (in the order of $\mathrm{MHz}$ or more) render measurements susceptible to electromagnetic interferences (EMI) in the test environment and would require more specialized shielding.

For two parallel surfaces, this capacitance is inversely proportional to the gap distance. Hence the sensitivity of the capacitance method is not linear, but becomes increasingly 
sensitive as the gap reduces. This is particularly relevant from a tribological perspective, since the precision of the measurement becomes more important as the electrode gap or film thicknesses approach nanometer dimensions. As with optical interferometry methods, macrotribological contacts can be studied. It is interesting to note that the optical interferometry method was developed in roughly the same time period. It was naturally favoured over the capacitance method for the measurement of EHL film thickness, since a direct observation of the film thickness profile over the entire contact can be made, whereas the capacitance method can only provide an average value for the film thickness. The advantage of the capacitance method over the optical method, however, is that both the surfaces can be made of metal, whereas the latter requires one surface to be a transparent material coated with a thin chromium layer. The method is hence more resilient to the effects of wear between the sliding surfaces - a prerequisite for their study of tribochemical reactions.

The greatest limitation of the capacitance method is that the dielectric constant of the lubricant film in the area of contact must be known to convert capacitance values to meaningful film thickness values. Accuracy of the method is therefore highly dependant on the accuracy of the measured or calculated dielectric constant of the fluid at the relevant contact pressures. The dielectric constant of a fluid is mostly dependent on temperature and pressure. While the same is true for the refractive index (which is needed for optical inteferometry film thickness measurements), it is generally less affected by changes in these conditions. The dielectric constant of mineral oils at ultra high pressures, was measured by Galvin et al. [47] using a high pressure condenser. They can also be predicted using empirical methods, which relate the dielectric constant as a function of pressure and its density. The methods chosen for this work are discussed in detail in Section 3.3. 


\subsection{SUMMARY}

Below is a summary of the key points in the literature review, which provide the basis of the research going forward.

1. The current model for the boundary lubrication mechanism of plant oils is similar to that of the fatty acids, except that the latter chemically adsorbs onto steel surfaces, while the former physically adsorbs. While this seems to be a very simple model for the boundary lubrication mechanism of bio-based lubricants, later experiments conducted by other researchers reveal that the mechanisms at work could be far more complex. Limitations of our understanding are clearly evidenced in the works of Murakami et al. and Sahoo et al., whereby polyunsaturated lipids were shown to perform better under high temperature conditions, and in long term tribological conditions respectively.

2. Polyunsaturated plant oils have poor oxidation stability due the presence of their double bonds. The extreme conditions of high pressure, temperature and shear, conducive to oxidative reactions could be replicated in the tribological contacts for plant oil lubricated surfaces. While there has been a substantial amount of research in the field of polymers made from plant oils, few have worked on the possibility that such polymerization processes could be affected at the interface between rubbing surfaces, and possibly form products that could possibly reduce friction and wear. The concept of generating polymeric films from the tribological process is termed as tribopolymerization.

3. Since the classical work by Bowden and Tabor on fatty acids, our understanding of the boundary lubrication of plant oils is limited at the initial state of molecular adsorption. Very little is known about the tribochemical reactions that occur when plant oil boundary films are subjected to prolonged high contact pressures and shear forces. 
This gap in our knowledge is partially due to the limitations of current state-of-the-art film measurement techniques. It is essential that film thickness be measured in conjunction with friction for a conclusive analysis of the results. Among all the methods reviewed, the capacitance method of film thickness measurement would be the most suitable method for the purpose of this work, since it allows ultra-thin film thicknesses to be measured in lubricated metallic sliding contacts.

\section{REFERENCES}

1. Fox, N.J., Stachowiak, G.W.: Vegetable oil-based lubricants - A review of oxidation. Tribol. Int. 40, 1035-1046 (2007)

2. Wagner, H., Luther, R., Mang, T.: Lubricant base fluids based on renewable raw materials. Their catalytic manufacture and modification. Applied Catalysis A: General 221, 429-442 (2001)

3. Bowden, F.P., Tabor, D.: The Friction and Lubrication of Solids. Oxford University Press Inc. New York (1950)

4. Adhvaryu, A., Erhan, S.Z., Perez, J.M.: Tribological studies of thermally and chemically modified vegetable oils for use as environmentally friendly lubricants. Wear 257, 359-367 (2004).

5. Bhuyan, S., Sundararajan, S., Yao, L., Hammond, E.G., Wang, T.: Boundary lubrication properties of lipid-based compounds evaluated using microtribological methods. Tribol. Int. 22(2), 167-172 (2006)

6. Zhang, P.Y., Xue, Q.J., Du, Z.L., Zhang, Z.J.: The tribological behaviours of ordered system ultrathin films. Wear 254, 959-964 (2003) 
7. Pooley, C.M., Tabor, D.: Friction and molecular structure: The behaviour of some thermoplastics" Proc. R. Soc. Lond. A 329, 251-274 (1972)

8. Cho, Y-K., Granick, S.: Shear of confined perfluorinated molecules: Effect of side branching. Wear 200, 346-352 (1996)

9. Hsu, S.M.: Boundary lubrication: current understanding. Tribol. Lett. 3, 1-11 (1997)

10. Spikes, H.A.: Direct observation of boundary layers. Langmuir 12, 4567-4573 (1996)

11. Biresaw, G.: Elastohydrodynamic properties of seed oils. J. Am. Oil Chem. Soc. 83(6), 559$566(2006)$

12. Biresaw, G., Bantchev, G.: Effect of chemical structure on film-forming properties of seed oils. J. Syn. Lubri. 25, 159-183 (2005)

13. Luo, J.B., Wen, S.Z., Huang, P.: Thin film lubrication. Part I: Study on the transition between EHL and thin film lubrication using a relative optical interference intensity technique. Wear 194, 107-115 (1996)

14. Yoshizawa, H., Israelachvii, J.N.: Fundamental mechanisms of interfacial friction. 2. stickslip friction of spherical and chain molecules. J. Phys. Chem. 97, 11300-11313 (1993)

15. Murakami, T., Sakamoto, H.: Lubricating properties of vegetable oils and paraffinic oils with unsaturated fatty acids under high-contact pressure conditions in four-ball tests. $J$. Synth. Lub. 20(3), 183-277 (2003)

16. Sahoo, R.R., Biswas, S.K.: Frictional response of fatty acids on steel. J. Colloid Interface Sci. 333(2), 707-718 (2009)

17. Fox, N.J., Stachowiak, G.W.: Boundary lubrication properties of oxidized sunflower oil. Lubrication Engineering 59(2), 15-20 (2003) 
18. Fox, N.J., Stachowiak, G.W.: Boundary lubrication performance of free fatty acids in sunflower oil. Tribol. Lett. 16(4), 275-281 (2004)

19. Furey, M.J.: The formation of polymeric films directly on rubbing surfaces to reduce wear. Wear 26, 369-392 (1973)

20. Furey, M.J., Kajdas, C., Ward, T.C., Hellgeth, J.W.: Thermal and catalytic effects on tribopolymerization as a new boundary lubrication mechanism. Wear 136, 85-97 (1990)

21. Furey, M.J., Kajdas, C., Kempinski, R.: Application of the concepts of tribopolymerization in fuels, lubricants, metalworking and 'minimalist' lubrication. Lubri. Sci. 15, 73-82 (2002)

22. Kajdas, C., Furey, M.J., Kempinski, R., Valentino, J.: New condensation-type monomer combinations as ashless antiwear compositions. Wear 249, 235-239 (2001)

23. Zheng, J., Zhang, L., Du, Z., Zhang, C., Li, H.: Tribopolymerization of n-butyl acrylate on the steel-steel rubbing surface. Tribol. Int. 41, 769-777 (2008)

24. Svahn, F., Csillag, S.: Formation of low-friction particle/polymer composite tribofilms by tribopolymerization. Tribol. Lett 41, 387-393 (2011)

25. Seniha Güner, F., Yağci, Y., Tuncer Erciyes, A.: Polymers from triglyceride oils. Prog. Polym. Sci. 31, 633-670 (2006)

26. Narine, S.S., Kong, X.: Vegetable oils in production of polymers and plastics. In Shahidi, F. (ed), Bailey's Industrial Oil and Fat Products., John Wiley and Sons, Inc., new Jersey, pg 279-306.(2005)

27. Korus, R.A., Mousetis, T.L.: Polymerization of safflower and rapeseed oils. J. Am. Oil Chem. Soc. 61, 537-540 (1984)

28. Meier, M.A.R., Metzger, J.O., Schubert, U.S.: Plant oil renewable resources as green alternatives in polymer science. Chem. Soc. Rev. 36, 1788-1802 (2007) 
29. Gohar, G., Cameron, A.: Optical measurement of oil film thickness under ehd lubrication. Nature 200, 458-459 (1963)

30. Spikes, H.A., Cann, P.M.: The development and application of the spacer layer imaging method for measuring lubricant film thickness. Proc. Instn. Mech. Engrs. Part J: J. Eng. Tribol. 215, 261-277 (2001)

31. Westlake, F., Cameron, A.: A study of ultra-thin lubricant films using wedged spacer layer optical interferometry. In D. Dowson et al. (eds), Interface Dynamics, Proceedings of the $14^{\text {th }}$ Leeds-Lyon Symposium on Tribology. Elsevier, Amsterdam, (1987, 1988)

32. Johnston, G.J., Wayte, R., Spikes, H.A.: The measurement and study of very thin lubricant films in concentrated contacts. Tribol. Trans. 34(2), 187-194 (1991)

33. Hartl, M., Krupka, I., Poliscuk, R., Liska, M.: Thin film colorimetric interferometry. Tribol. Trans. 44, 270-276 (2001)

34. Glovnea, R.P., Forrest, A.K., Olver, A.V., Spikes, H.A.: Measurement of sub-nanometer lubricant films using ultra-thin film interferometry. Tribol. Lett. 15(3), 217-230 (2003)

35. Spikes, H.A.: The borderline of elastohydrodynamic lubrication and boundary lubrication. Proc. Instn. Mech. Engrs. Part C: J. Mech. Eng. Sci. 214, 23-37 (2000)

36. Rugar, D., Hansma, P.: Atomic force microscopy. Physics Today 43(10), 23-30 (1990)

37. Binnig, G., Quate, C.F.: Atomic force microscope. Phys. Rev. Lett. 56(9), 930-933 (1986)

38. Bhushan, B.: Introduction to Tribology. John Wiley \& Sons, New York (2002)

39. Beech, I.B., Smith, J.R., Steele, A.A., Penegar, I., Campbell, S.A.: The use of atomic force microscopy for studying interactions of bacterial biofilms with surfaces. Colloids Surf B: Biointerfaces 23, 231-247 (2002) 
40. Drake, B., Prater, C.B., Weisenhorn, A.L., Gould, S.A.C., Albrecht, T.R., Quate, C.F., Cannel, D.S., Hansma, H.G., Hansma, P.K.: Imaging crystals, polymers, and processes in water with the atomic force microscope. Science 243, 1586-1589

41. Homola, A.M., Israelachvili, J.N., Gee, M.L., McGuiggan, P.M.: Measurements of and relation between the adhesion and friction of two surfaces by molecularly thin liquid films. J. Tribol. 111(4), 675-682 (1989)

42. Heuberger, M., Luengo, G., Israelachvili, J.N.: Tribology of shearing polymer surfaces. 1. Mica sliding on polymer (PnBMA). J. Phys. Chem. B 103, 10127-10135 (1999)

43. Israelachvili, J.: Direct measurements of forces between surfaces in liquids at the molecular level. Proc. Natl. Acad. Sci. US A 84, 4722-4724 (1987)

44. Israelachvili, J., Min, Y., Akbulut, M., Alig, A., Carver, G., Greene, W., Kristiansen, K., Meyer, E., Pesika, N., Rosenberg, K., Zeng, H.: Recent advances in the surface force apparatus (SFA) technique. Rep. Prog. Phys. 73, 1-16 (2010)

45. Crook, A.W.: The Lubrication of rollers, Phil. Trans. Roy. Soc London A. 250(981), 387-409 (1958)

46. Dyson, A., Naylor, H., Wilson, A.R.: The measurement of oil-film thickness in elastohydrodynamic contacts. Proc. Instn. Mech. Engrs. Part 3B 180, 119-134 (1965)

47. Galvin, G.D., Naylor, H., Wilson, A.R.: The effect of pressure and temperature on some properties of fluids of importance in elastohydrodynamic lubrication. Proc. Instn. Mech. Engrs 178, 283-308 (1964) 


\section{Capacitance Method for Film Thickness Measurement}

\subsection{INTRODUCTION}

The literature review revealed several gaps in our understanding of plant oil boundary films, particularly in the effects of sliding distance on their tribological behaviour. This is partially attributed to the limitations of current film thickness measurement methods. It was clear from the onset that the immediate task for this research work was to develop a new instrument, which would be capable of measuring the thickness and friction of boundary films over long periods in a macro-tribological setting. Among the methods reviewed in Chapter 2, it was decided that the capacitance method would be the most suitable for the purpose of our study. It allows the use of metals as the material for both counter surfaces, and allows tribochemical reactions to occur without being too sensitive to the effects of wear. In this chapter, a theoretical formulation for the capacitance method will be described. The design, development and experimental setup of the new tribological rig would be presented in Chapter 4.

\subsection{CAPACITANCE MODEL AND CALCULATION METHOD}

The film thickness calculation method used is similar to that proposed by Dyson et al. [1], which requires assumption of the shape, and hence area of the contact zone. The capacitance 
of the ball can be evaluated in two parts, i.e. the capacitance at the Hertzian contact and the capacitance outside the contact. Dyson et al. [1] assumed that the Hertzian contact is a perfectly smooth and flat area in the study of EHL film thickness (see Fig. 3.1). For boundary lubrication, however, this assumption cannot be used, because the area of contact does not remain constant during sliding, and the surfaces will not be perfectly smooth. Whilst most boundary lubricants reduce the wear rate, they cannot prevent wear completely during the course of sliding. Some lubricant films may be sheared away, only to re-form at a later time, during which wear may take place in between; while others may require a longer period of running-in before a sustainable film is formed. In either case, the assumption of the Hertzian area of contact based on the original geometry of the surfaces will no longer be valid. This means that the wear scar diameter during the course of sliding must be known for the calculation of the film thickness to be accurate.

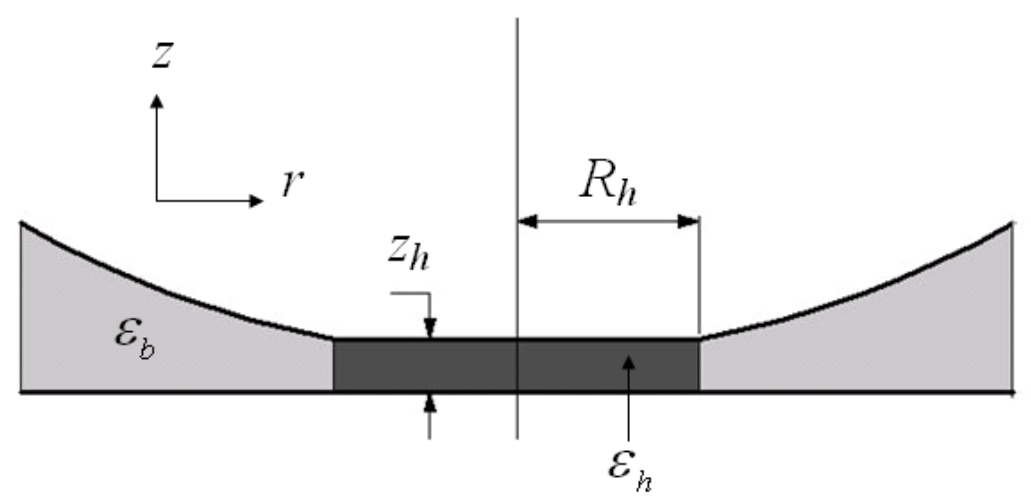

Fig. 3.1: Capacitance-film thickness model based on Dyson et al's [1] method, where $z_{b}=$ film thickness, $R_{b}=$ radius of the Hertzian contact area, $\varepsilon_{b}=$ bulk fluid dielectric constant, and $\varepsilon_{b}=$ the dielectric constant of the fluid at Hertzian contact pressure.

\subsubsection{Capacitance Contribution Within the Apparent Area of Contact}

According to Johnson [2], for two randomly rough surfaces in contact, the ratio of the real area of contact to the apparent area of contact is directly proportional to the mean apparent contact pressure, i.e.: 


$$
\frac{A_{r}}{A_{a}}=\pi^{1 / 2}\left(\sigma \kappa_{s}\right)^{-1 / 2}\left(\frac{p_{a}}{E^{\prime}}\right)
$$

where $A_{r}$ is the real area of contact, $A_{a}$ is the apparent area of contact, $\sigma$ is the surface roughness standard deviation, $x_{s}$ is the mean curvature of the surface profile, $p_{a}$ is the average apparent contact pressure, and $E^{\prime}$ is the reduced Young's modulus. It can be seen from eqn. (1) that, for a constant surface roughness, the real area of contact is directly proportional to the load, i.e. $A_{r} \propto p_{a} A_{a}$. It follows that, for a constant load, if the apparent area of contact increases due to wear, the mean apparent contact pressure must decrease accordingly, so that $A_{r}$ remains roughly the same during the sliding process. As long as the surface roughness is not significantly altered during sliding, this assumption will hold true. For this reason, the surfaces used in this work have been highly polished, so that running-in wear and the change in surface roughness during rubbing are not significant.

(a)

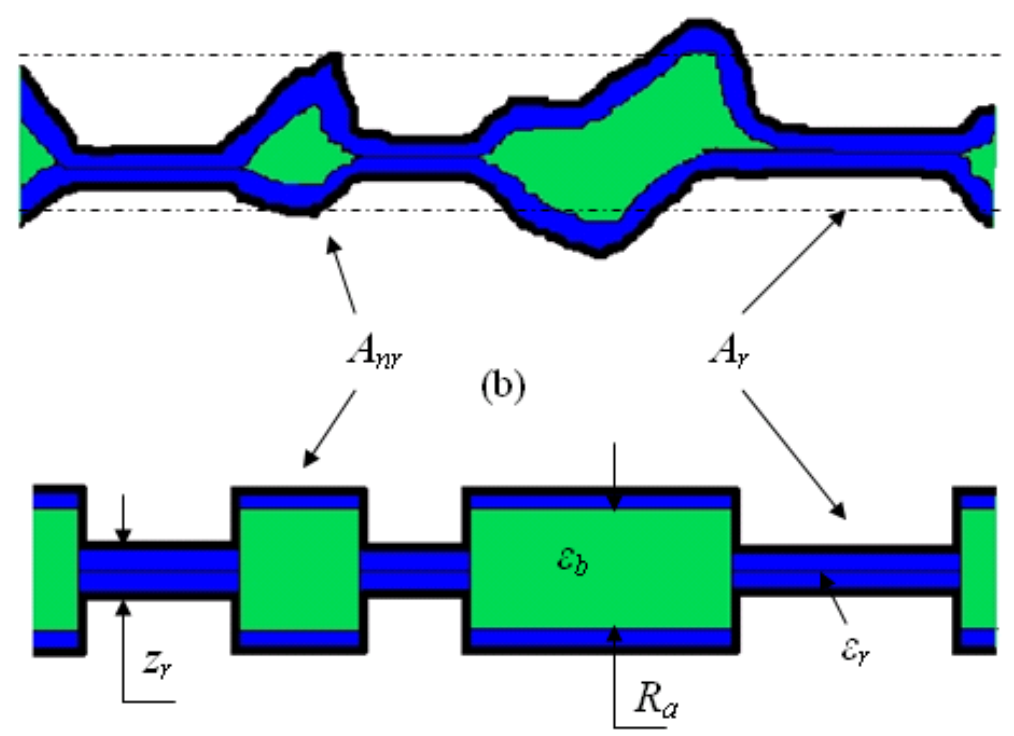

Fig. 3.2: a) Realistic contact between sliding surfaces; b) Modified capacitance-film thickness model at the apparent area of contact. 
As schematically illustrated in Fig. 3.2a, the asperities are deformed at the real areas of contact. In the presence of a boundary film, the opposing asperities would be separated by a distance equal to the thickness of the boundary film. The other areas not in contact (within the apparent area), $A_{n r}=A_{a}-A_{r}$ can be modelled as being separated by a composite film of two boundary layers and the entrapped bulk fluid. For simplicity, we assume that the average separation is equivalent to the sum of the boundary film thickness, $r$, and the combined surface roughness, $R_{a}$, of the two surfaces, as shown in Fig. 3.2b. Sellgren et al. [3] have shown that for smooth surfaces and high loads, the ratio $A_{r} / A_{a}$ approaches unity, so $A_{r}$ can be calculated from Hertzian contact equations. Since mirror polished surfaces and moderate Hertzian pressures of $0.69 \mathrm{GPa}$ were used in this study, it can be assumed that $A_{r} / A_{a}=1$ at the beginning of sliding, and the capacitive contribution of the boundary film at the real areas of contact is:

$C_{r}=\frac{\varepsilon_{r} \varepsilon_{o} A_{r}}{z_{r}}$

where, $A_{r}$ is the Hertzian contact area; $\varepsilon_{r}$ is the dielectric constant of the boundary layer at the average Hertzian pressure and test temperature; $\varepsilon_{0}$ is the permittivity of free space; and $z_{r}$ is the thickness of the boundary film.

The capacitance contribution of the composite film at the other areas within the apparent area of contact will be:

$$
C_{n r}=\frac{\varepsilon_{r} \varepsilon_{b} \varepsilon_{o} A_{n r}}{\varepsilon_{b} z_{r}+\varepsilon_{r} R_{a}}
$$

where, $\varepsilon_{b}$ is the dielectric constant of the bulk fluid at atmospheric pressure, $A_{n r}$ is the noncontact areas within the apparent area of contact, and $R_{a}$ is the combined surface roughness. To calculate $A_{n m}$, the apparent area of contact, $A_{a}$ at the time of rubbing must be known. This 
is done by monitoring the growth of the wear track width during the test. The wear track widths provide an estimate of the ball wear scar diameter and hence $A_{a}$ where $A_{n r}=A_{a}-A_{\text {r }}$.

It can be seen from eqn. (3) that if the boundary film thickness, $z_{r}$ is comparatively larger than the surface roughness, $R_{a}\left(z_{r}>>R_{a}\right)$, eqn. (3) reduces to $C_{n r}=\varepsilon_{0} \varepsilon_{t} A_{n r} / z_{r}$ Practically, there will be little wear for thick boundary films, so the capacitive contribution of $C_{n r}$ would be negligible since $A_{n r}$ is small. On the other hand, if $z_{r}<<R_{a}$, eqn. (3) reduces to $C_{n r}=\varepsilon_{o} \varepsilon_{b} A_{n r}$ / $R_{a^{*}}$. In this case, since the load will be mainly supported by the asperities at the real areas of contact, the local pressures at the non-contact areas must be close to the ambient pressure. For thin films, where the impact of wear cannot be ignored, $A_{n r}$ could be much greater than $A_{n}$, but since $\mathrm{R}_{a}$ is large compared to $z$, the total capacitance will, in effect, still be dominated by $C_{r}$.

\subsubsection{Capacitance Contribution Outside the Apparent Area of Contact}

Outside the apparent area of contact, the capacitance is modeled by a half sphere (see Fig. 3.3). The capacitive contribution outside the Hertzian contact, $C_{b}$ is thus calculated by summing the capacitive contribution of each infinitesimal area of the probe, $2 \pi r d r$. For a non-deformed bearing ball (with radius $R_{b}$ ) in contact with the flat disc is, the vertical coordinate, $z$, at any point along the radius, $r$, of the ball is:

$$
z=R_{b}-\sqrt{R_{b}^{2}-r^{2}}
$$

Under the test load, this gap is reduced by the wear depth of the ball, $z_{w}$ and raised by the boundary film thickness, ₹, as shown in Fig. 3.3. Hence,

$$
C_{b}=\int_{R_{w}}^{R_{b}} \frac{2 \pi r \cdot \varepsilon_{b} \varepsilon_{o}}{\left(R_{b}-\sqrt{R_{b}^{2}-r^{2}}\right)-z_{w}+z_{r}} d r
$$


where $\varkappa_{y}$ is the wear depth of the ball and $R_{w}$ is the radius of the wear scar. At sliding time, $t=$ $0, z_{w}=q_{d}$, and $R_{w}=R_{b}$, where $q_{d}$ is the Hertzian maximum deflection of the ball under the test load and $R_{b}$ is the radius of the Hertzian contact area.

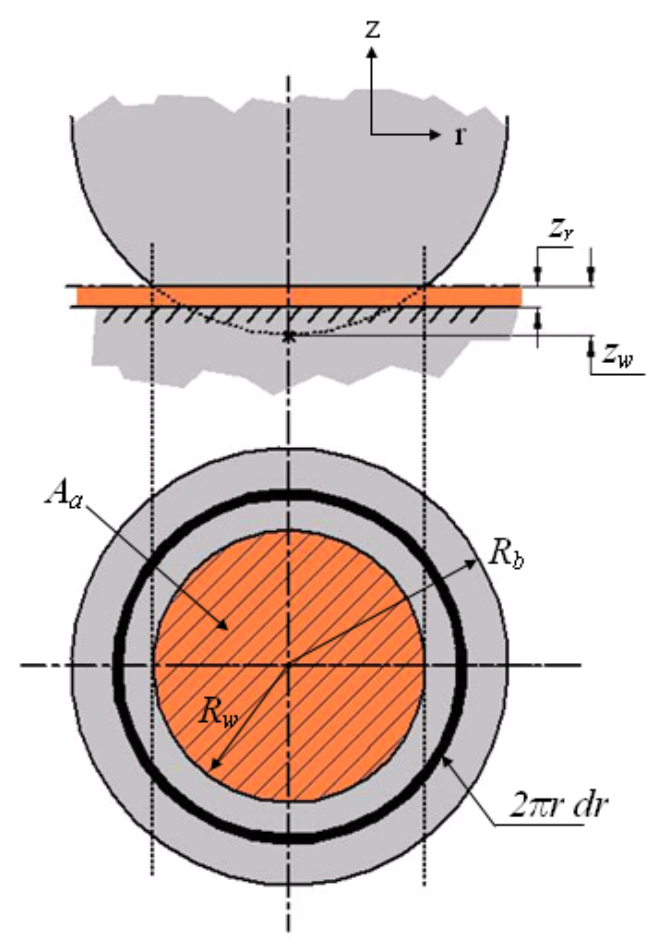

Fig. 3.3: Schematic illustration of the capacitance contribution outside the apparent area of contact. Note that the apparent area of contact has been exaggerated for clearer annotation.

\subsubsection{Conversion of Capacitance Values to Film Thickness Values}

The total capacitance, $C_{t}$ is simply the sum of the capacitances derived above:

$$
C_{t}=C_{r}+C_{n r}+C_{b}
$$

Once the dielectric constants of the test lubricant at atmospheric and contact pressure have been determined, the predicted capacitances can then be calculated for film thicknesses varying from $0.1 \mathrm{~nm}$ to $0.1 \mu \mathrm{m}$, and for wear scar diameters varying from the initial Hertzian contact radius of $56.3 \mu \mathrm{m}$ (current work) to $250 \mu \mathrm{m}$. This was done using MATLAB. An example of the capacitance-film thickness relationship for sunflower oil is shown in Fig. 3.4, 
using an iterative step size of $1 \mu \mathrm{m}$ and $1 \mathrm{~nm}$ for the wear scar radius and the film thickness respectively. After the test, the recorded capacitance values were then converted to film thickness values by interpolation of the 3D relationship using MATLAB given time associated inputs of the wear track width.

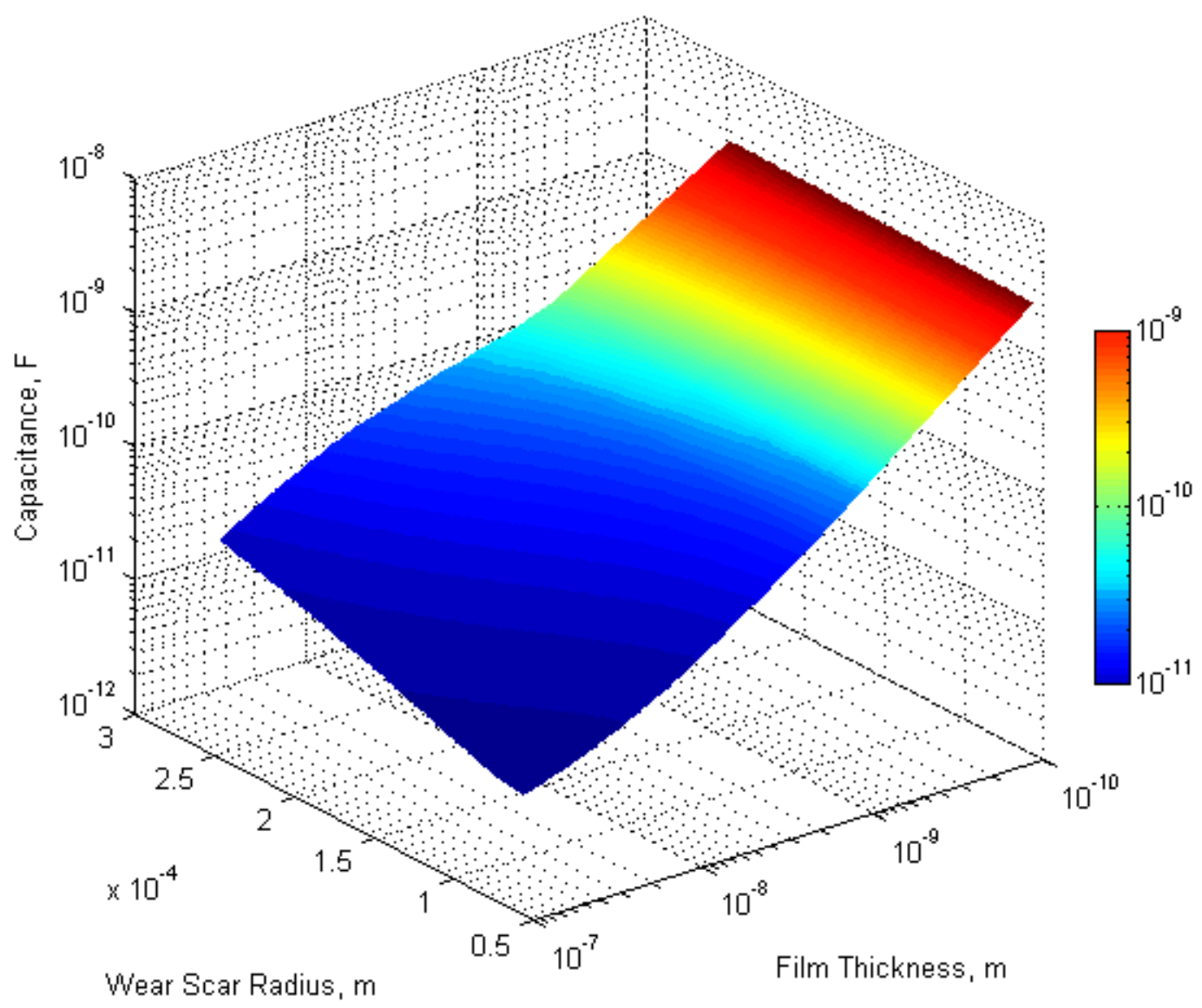

Fig. 3.4: Capacitance-film thickness-wear scar radius relationship for sunflower oil

\subsection{PREDICTION OF THE DIELECTRIC CONSTANT, \& AT HIGH PRESSURES}

The capacitance method requires that the dielectric constant of the lubricant film between the sliding contacts to be known before the film thickness can be calculated. This can be done experimentally with the use of a high pressure condenser, or by employing empirical methods. 
In order to use empirical methods, one must know the dielectric constant of the test lubricant under ambient pressure and at specified temperature. This can be easily accomplished by using the capacitance cell in the newly developed tribometer, which would be presented in the Chapter 4. With knowledge of the dielectric constant under ambient pressures, it is possible to utilise empirical methods to determine the dielectric constant at the contact pressure for a given temperature. These empirical methods are based on previous work done by other researchers using fluids of similar dielectric constants.

The dielectric constants for the fluids tested in Dyson et al.'s [1] work was measured by Galvin et al. [4] The latter compared experimental values with theoretical prediction using the well-known Clausius-Mosotti (CM) equation:

$$
\frac{\varepsilon-1}{\varepsilon+2}=\rho \cdot Y_{o}
$$

where $Y_{0}$ is a constant. They found that the $\mathrm{CM}$ equation is relatively accurate for non-polar fluids (such as mineral oils). With polar liquids however, such as fatty acids and ester lubricants, the method considerably overestimates the dielectric constant, particular at high pressures. The general consensus on this discrepancy is that the CM equation does not take into account the fact that the polarizability of a polar molecule does not vary linearly with density. A more appropriate formula for polar fluids was derived by Onsager [5]:

$$
\frac{\left(\varepsilon-\varepsilon_{\infty}\right)\left(2 \varepsilon+\varepsilon_{\infty}\right)}{\varepsilon\left(\varepsilon_{\infty}+2\right)^{2}}=\rho \cdot Y_{1}
$$

where $Y_{1}$ is a constant and $\varepsilon_{\infty}$ is the dielectric constant at high frequencies, which is usually approximated using the Maxwell relation $\varepsilon_{\infty}=n^{2}$, where $\mathrm{n}$ is the refractive index. Good correlation between experimental data and calculation was found for moderately polar liquids $(\varepsilon<5)$ by Valisko et al. [6] using the Onsager equation. Since most boundary lubricants would 
either be a pure polar liquid or a blend of polar components in a non-polar liquid, the Onsager equation would be more suitably applied.

Both eqn. 7 and 8 require the density at the Hertzian contact pressure to be known before the dielectric constant can be calculated. A convenient formula commonly used in the field of elastohydrodynamic lubrication relating density to pressure is the formula by Dowson and Higginson [7]: $\varrho / \varrho_{o}=1+[0.6 p /(1+1.7 p)]$. However, it has been shown by several workers that this equation tends to overestimate the density, particularly at high pressures [8]. The equation of state generally regarded as the most accurate, comes in the form of the modified Tait-Tammann equation [9]:

$$
\rho=\frac{\rho_{o}}{1-C \ln \left(\frac{B+p}{B+p_{o}}\right)}
$$

, where $B$ and $C$ are constants, $p_{0}$ is the ambient pressure, $p$ is the pressure of interest, and $\varrho_{0}$ is the density of the fluid at ambient pressure. Bair [10] has also recommended the use of this formula for the quantitative study of EHL contacts. The $B$ and $C$ constants for thirteen natural fats and oils, and two pure triglycerides (triolein and trilarin) were evaluated by G.M Acosta et al [11] at $30,40,60$ and $80^{\circ} \mathrm{C}$, and the constants $\mathrm{B}=110$ and $\mathrm{C}=0.07325$ has been chosen for this current work.

\section{REFERENCES}

1. Dyson, A., Naylor, H., Wilson, A.R.: The measurement of oil-film thickness in elastohydrodynamic contacts. Proc. Instn. Mech. Engrs. Part 3B 180, 119-134 (1965)

2. Johnson, K.L.: Contact Mechanics. Cambridge University Press, Melbourne, Australia (1987)

3. Sellgren, U., Björklund, S., Andersson, S.: A finite element-based model of normal contact between rough surfaces. Wear 254, 1180-1188 (2003) 
4. Galvin, G.D., Naylor, H., Wilson, A.R.: The effect of pressure and temperature on some properties of fluids of importance in elastohydrodynamic lubrication. Proc. Instn. Mech. Engrs. Part 3N 178, 283-290 (1963)

5. Onsager, L.: Electric moments of molecules in liquids. J. Am. Chem. Soc. 58, 1486-1493 (1936)

6. Valisko, M., Boda, D.: Relative permittivity of polar liquids. Comparison of theory, experiment and simulation. J. Phys. Chem. B 109, 6355-6365 (2005)

7. Dowson, D., Higginson, G.R.: Elastohydrodynamic lubrication. Pergamon Press, Oxford (1966)

8. Wong, P.L., Wang, R., Lingard, S.: Pressure and temperature dependence of the density of liquid lubicants. Wear 201(1), 58-63 (1996)

9. Tammann, G.: The dependence of the volume of solutions on pressure. Z. Phys. Chem. Stoechiom. Verwandtschafts 17, 620-636 (1895)

10. Bair, S.: Rheology and high-pressure models for quantitative elastohydrodynamics. Proc. Instn. Mech. Engrs. Part J: J. Eng. Tribol. 223(4), 617-628 (2009)

11. Acosta, G.M., Smith, Jr., R.L., Arai, K.: High-pressure PVT behavior of natural fats and oils, trilaurin, triolein, and n-tridecane from $303 \mathrm{~K}$ to $353 \mathrm{~K}$ from atmospheric pressure to 150 MPa. J. Chem. Eng. Data 41, 961-969 (1996) 


\section{Development of the Contact Impedance Tribometer}

\subsection{INTRODUCTION}

In this chapter, the design and development of the new instrument, named the contact impedance tribometer (CIT), is presented, along with a pilot test using sunflower oil. The instrument is capable of simultaneously measure the friction, wear and thickness of boundary films down to $1 \mathrm{~nm}$.

It was decided at the conceptual stages of the design to keep the tribometer as small as possible. Having a small setup provides several advantages - 1) It would allow a smaller quantity of the test sample to be used; 2) control of the temperature during experiments would be more effective and accurate and; 3) errors in the measurements due to mechanical misalignments and tolerances would be minimized. Once the conceptual design was finalized, a detailed design using Solidworks, and the procurement of equipment, including the motor drive, reduction gears, transmission belts, LCR meter were undertaken in parallel. The instrument was wholly manufactured in-house at the School's workshop. The development process of the tribometer is summarized in the flow chart shown in Fig. 4.1. 


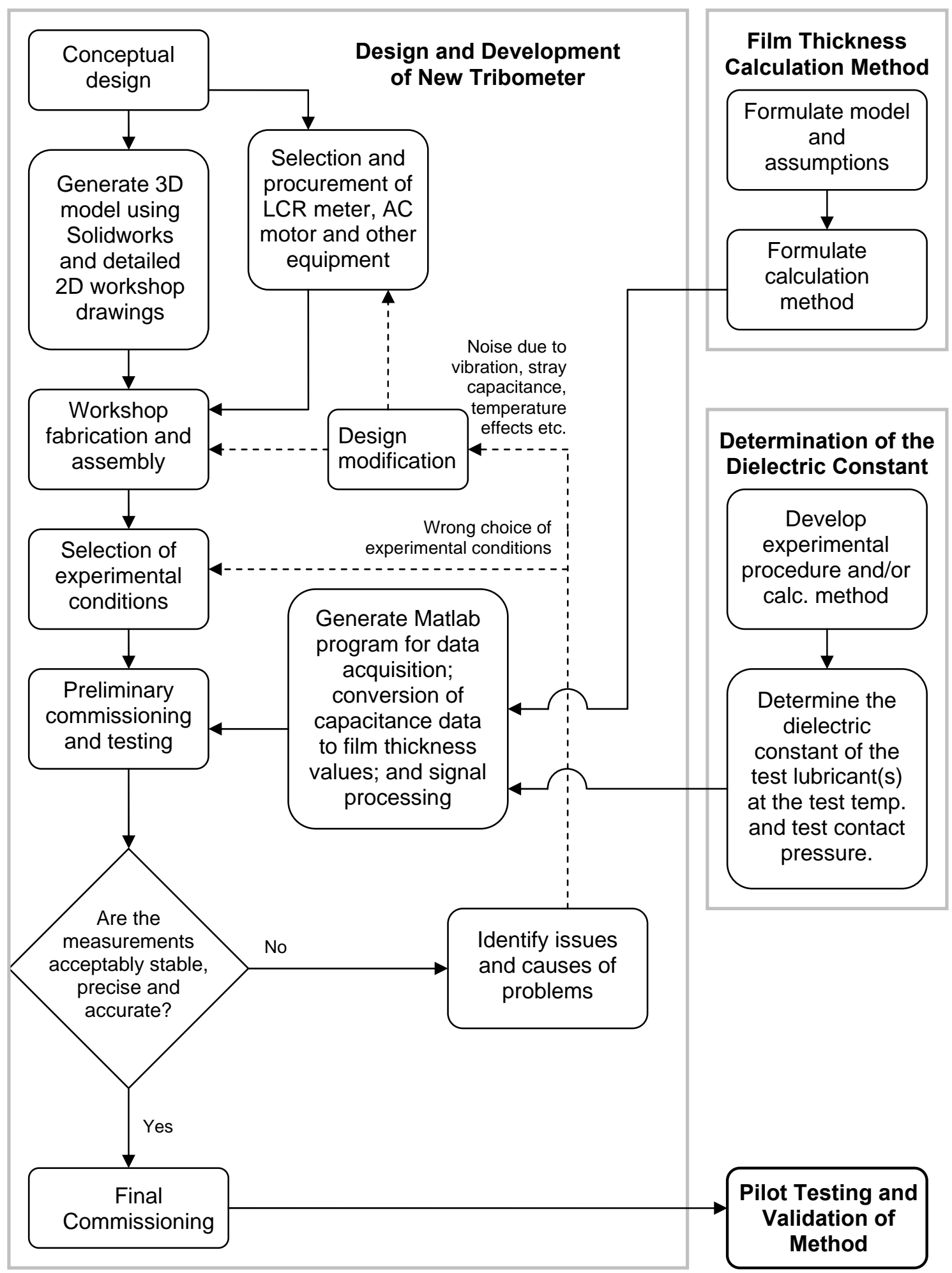

Fig 4.1: Flowchart showing the development of the contact impedance tribometer (CIT). 


\subsection{EXPERIMENTAL SETUP}

The experimental setup is shown in Fig. 4.2. The setup is basically that of a ball-on-disc tribometer. The main difference is that the ball holder is modified to act as the capacitance probe.
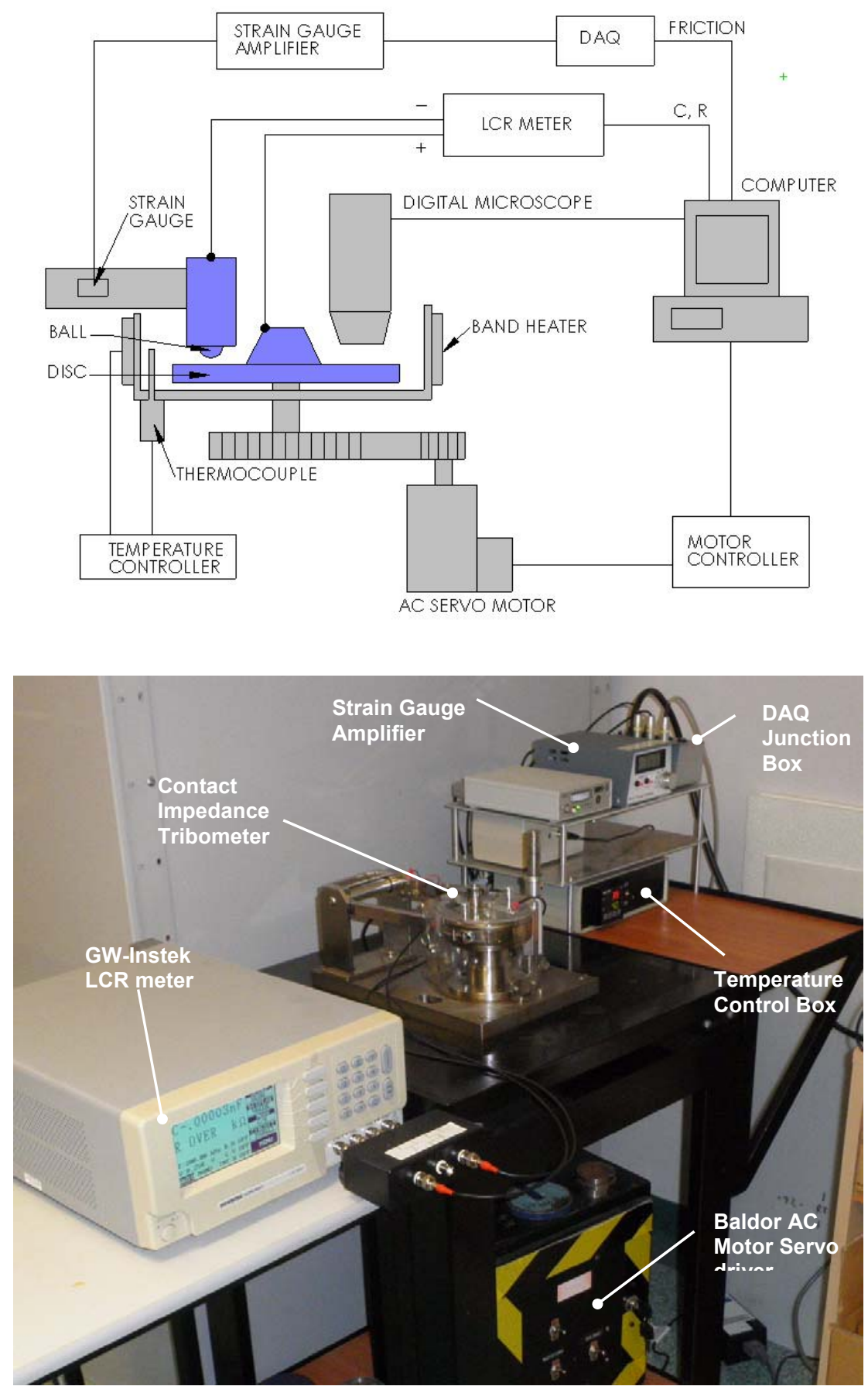

Fig. 4.2: a) Schematic illustration of the experimental setup. b) Photograph of the contact impedance tribometer (CIT) 


\subsubsection{Components}
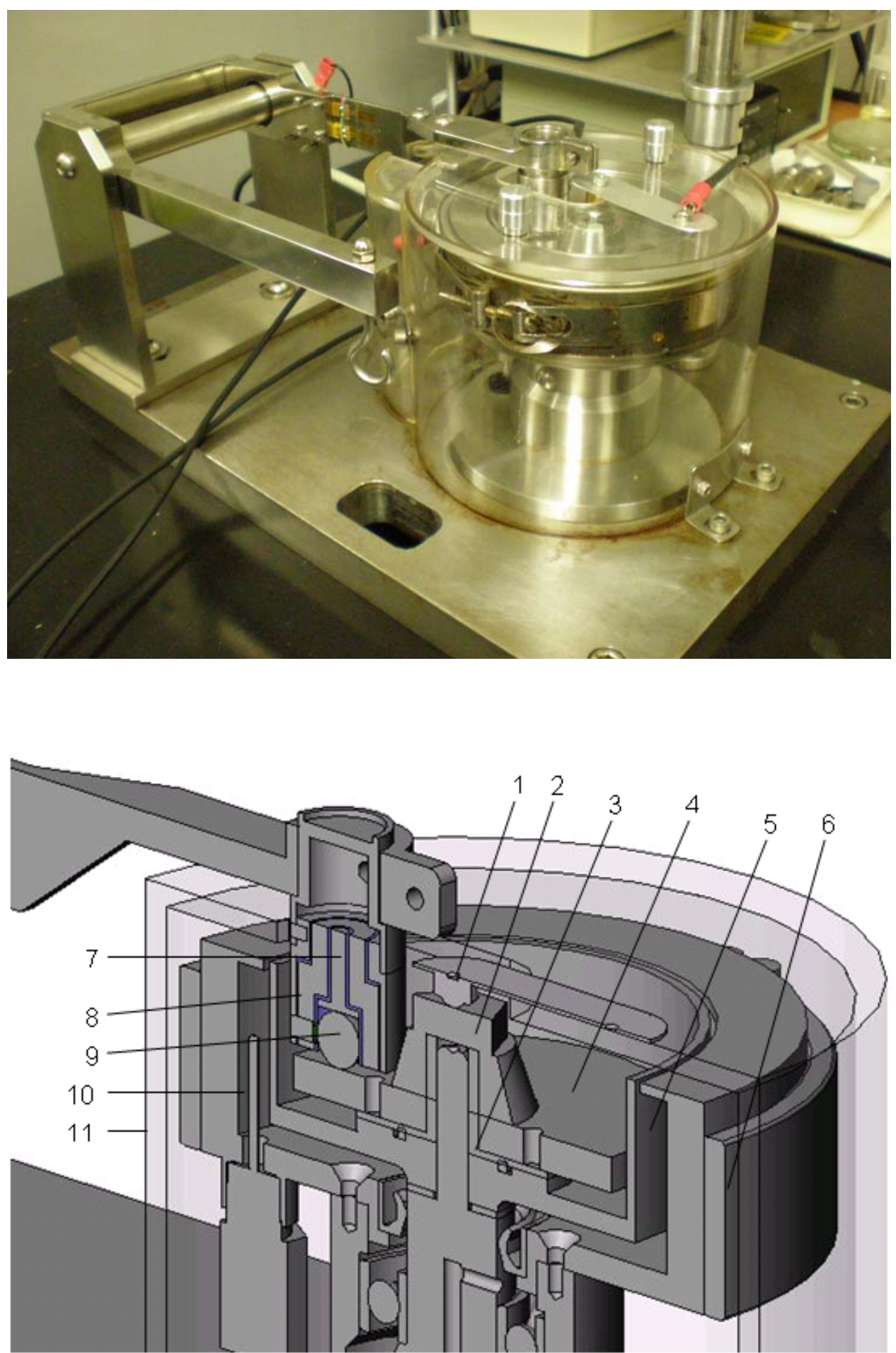

Fig. 4.3: a) Close-up photograph of the CIT; b) Sectional 3D view of test cell assembly. 1. Brass contact with cantilever spring; 2. securing nut; 3. electrical insulating sleeve; 4. test disc; 5 . heating oil chamber; 6 . band heater; 7. negative electrode; 8. guard ring; 9. 8mm diameter bearing ball; 10 . thermocouple; 11 . cover 


\section{Capacitance Probe}

The design of the probe is one of the most critical aspects of the development. The capacitance probe comprises of a guard ring (8) surrounding the bearing ball to eliminate fringing capacitance, thereby improving the accuracy of the measurement. The guard is in turn enclosed in an outer shield to avoid ground loops due to small differences in electrical ground between the rig and the meter. The guard ring surrounding the electrode stem must be sufficiently thick to reduce the effects of any parasitic capacitances. This is particularly important along the electrode stem and the region near the sliding ball.

The $8 \mathrm{~mm}$ diameter bearing ball (9) was secured via a nylon tipped grub screw, onto the negative electrode seat (7) of the capacitance probe. The bearing ball thus served as both the slider and the negative electrode for impedance measurements. The $8 \mathrm{~mm}$ diameter bearing ball used is a commercially available bearing ball with a surface roughness $R_{a}$ of $11 \mathrm{~nm}$ and hardness of 63-65 HRB.

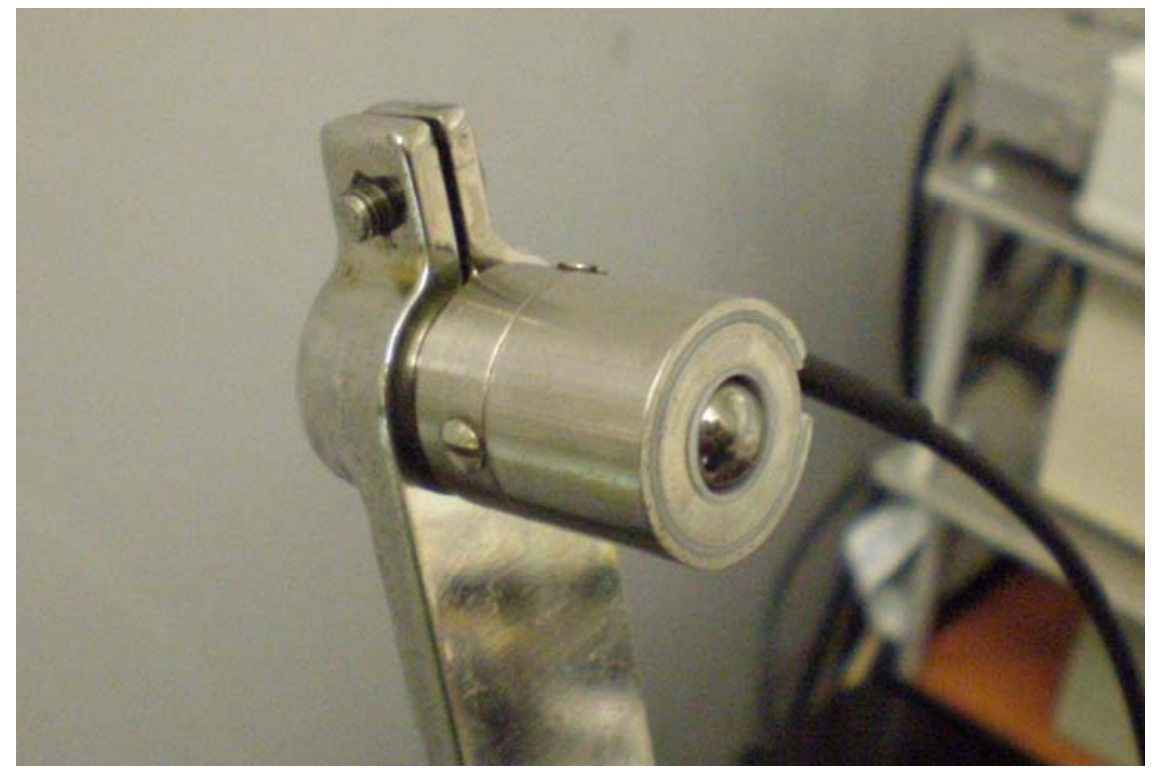

Fig: 4.4: Photograph of the Capacitance Probe, mounted on the cantilever arm 


\section{Rotating Disc and Electrode Assembly}

The counter surface is a flat $60 \mathrm{~mm}$ diameter disc (4), and served as the positive electrode. The rotating wear disc was electrically connected by the securing nut and brass contact (1), which was kept in constant contact using an aluminium cantilever spring. The disc is insulated from the rest of the capacitance cell by the use of a Teflon sleeve (3).

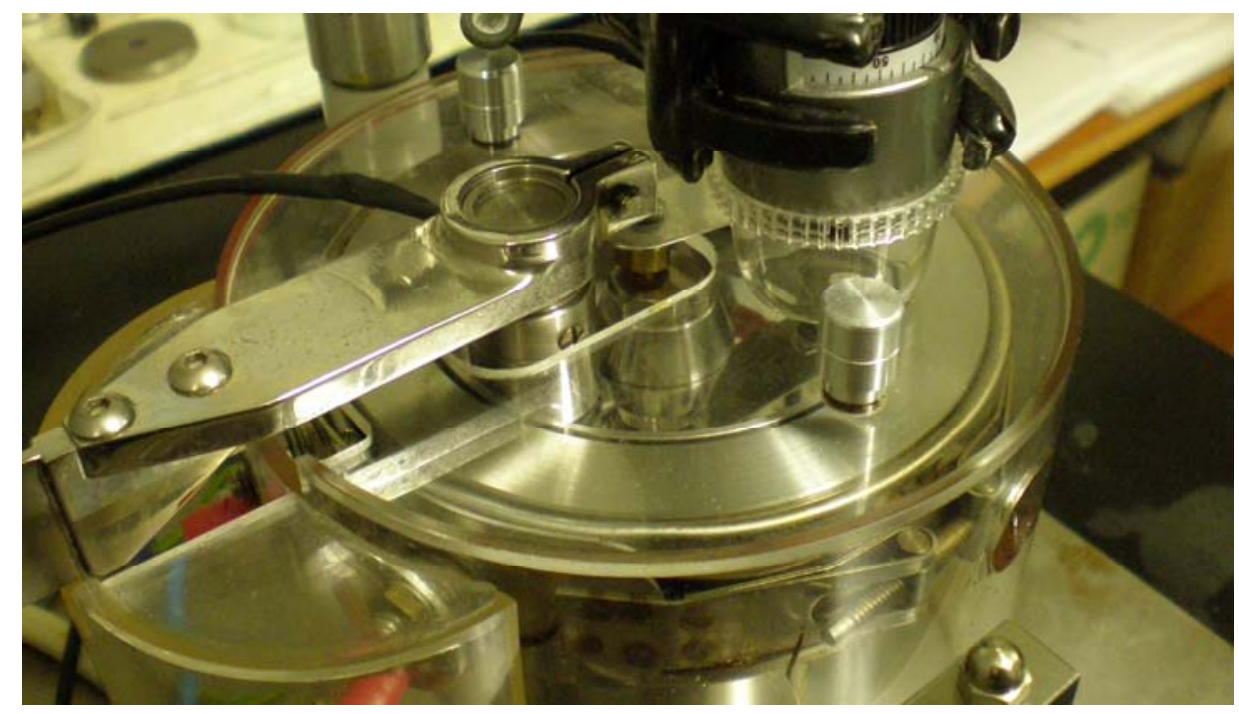

Fig. 4.5: Capacitance cell with cantilever arm and DinoCapture camera in position

During the initial testing of the newly developed rig, it was found that hardness of the rotating disc material was important in ensuring consistent results. Mild steel of approx $140 \mathrm{HV}$ was initially used as the material. However, this was found to be too soft against that of the sliding ball, which resulted in a large amount of deformation and ploughing during the sliding test. Impedance could not be measured due to the high amount of metallic wear particles that became entrapped in the contact area, causing short circuiting to occur. Hertzian contact analysis indicates that the contact stresses in our tests substantially exceed the yield strength of mild steel. To a lesser extent, the same was observed when the disc was rough. 
The issues were resolved when the material was changed to a 52100 steel, hardened to $810 \pm 10 \mathrm{HV}$ and then polished to a mirror finish of $13 \mathrm{~nm} \mathrm{R}_{\mathrm{a}}$. This was achieved by heat treating the steel to $860{ }^{\circ} \mathrm{C}$ for $20 \mathrm{~min}$, quenching it in vegetable oil and then tempering at 150 ${ }^{\circ} \mathrm{C}$ for another $20 \mathrm{~min}$. The low wear rate of the wear disc prevents short circuiting problems caused by wear particle entrapment and allows the changes in friction and film thickness due to sliding to be observed more progressively.

\section{Motor Drive and Transmission}

The rotating disc assembly is belt driven by a Baldor AC servomotor (Model No. BSM50N133AF). It has continuous stall torque of $0.45 \mathrm{Nm}$ and a maximum speed of $10,000 \mathrm{rpm}$. The servomotor is controlled via a Baldor Microflex AC servodriver (Model No. FMH2A03-TREN23). The drive reduction is 10, giving the instrument a range of 1-1000 rpm at the rotating disc. A disc speed of $10 \mathrm{rpm}$ corresponds to $20 \mathrm{~mm} / \mathrm{s}$ on a wear track radius of $20 \mathrm{~mm}$. Vibration from the AC servo motor is damped by mounting the entire instrument on a thick 1" base plate which sits on the supporting frame below via anti-vibration rubber mountings.

\section{Heating and Temperature Control}

Heating was achieved through the use of a Watlow thinband heater (6) clamped around the assembly housing, while temperature control was achieved by the use of a Watlow P\&ID basic temperature controller and thermocouple (10) mounted in the heating chamber (5). The heating chamber is filled partially with silicone oil, which serves as the heating oil to provide uniform heating. The lubricant cell was sealed from the heating chamber by an ' $\mathrm{O}$ ' ring, to prevent cross contamination of the test lubricant with the heating oil. This setup allows the cell to be removed easily for cleaning after the test. 


\section{Grounding and EMF Shielding}

The electrode assembly, the securing nut and the wear disc had been electrically insulated from the rest of the setup. Due to electromagnetic interference and parasitic capacitances from the AC servo motor, it is critical to ensure that instruments and mechanical components are properly grounded. Although both the capacitance cell and the body of the AC servo motor are separately grounded to the thick base plate, parasitic capacitances from the AC servo motor can still interfere with the sensitive impedance and frictional measurements through the motor mounting points on the thick base plate. This is remedied by changing the mounting of the AC servo motor to the base plate via 4 teflon mounting bushings. Half metre long RG174/U shielded coaxial cables were also used to connect the capacitance probe and the rotating disc to the LCR meter.

\subsubsection{Measurement Systems}

All capacitance and friction measurements are recorded simultaneously by a computer in a 'txt' format using their respective programs. A Matlab program was also created to process the data and convert the capacitance values into film thickness values.

\section{Friction Measurement}

Friction force was measured using strain gauges bonded onto the load cantilever arm. The strain gauges amplifier is connected to an AGILENT DAQ (model no.U2354A), which is controlled via a MATLAB Graphical User Interface (GUI) program (see Fig. 4.6). The GUI program has been created to allow the user to start and stop the data acquisition, input the experimental conditions and sampling parameters of each test. It also plots in real time, the friction measured against the speed of the rotating disc. The 'Oscilloscope' plots the signal voltage against time for one revolution of the rotating disc. 
The sensitivity of the friction force measurement is about $0.632 \mathrm{~N} / \mathrm{V}$, and provides frictional measurement with an accuracy of 0.002 at $1 \mathrm{~kg}$ load. In the case shown in Fig. 4.6, friction signals were sampled at 1000 samples per second, with a sampling block of 6000 samples per trigger. This corresponds to a sampling time of 6 seconds, which is the time taken for one disc revolution when the disc is set to rotate at $10 \mathrm{rpm}$. The 6000 samples taken over one revolution is then averaged, converted to frictional values, and then plotted on the 'Recorder', until the test reaches 10800 cycles as specified by the user or when the 'stop' button is clicked.

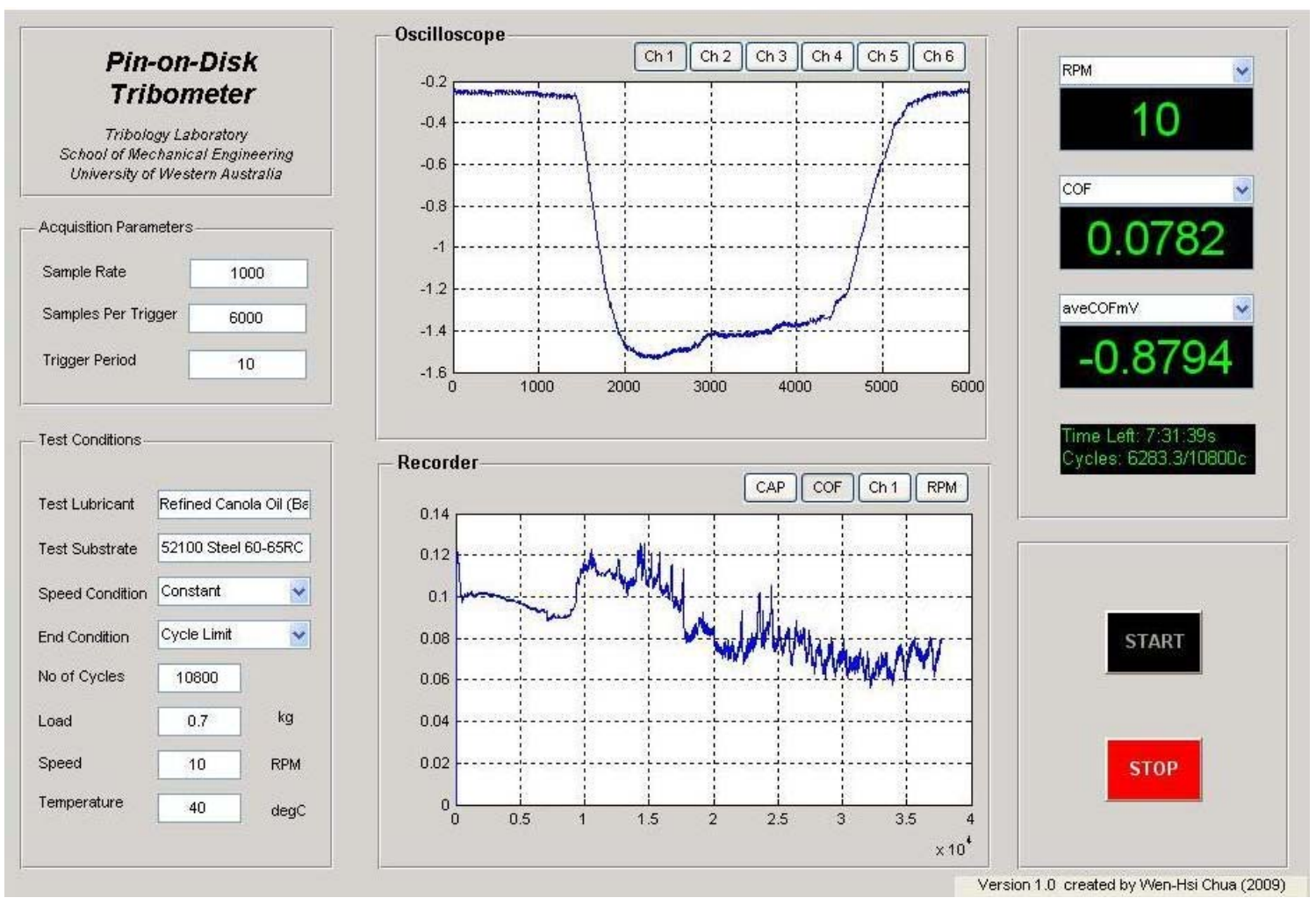

Fig. 4.6: Matlab GUI for frictional data acquisition

\section{Capacitance Measurement}

The capacitance and resistance measurements were made simultaneously using a GW-Instek LCR 821 meter. The excitation current used for the impedance measurements was $200 \mathrm{kHz}$ at $0.25 \mathrm{~V}$, which corresponds to the manufacturer's rated accuracy of approximately $1 \%$ of 
reading. Impedance readings from the meter are recorded separately on the computer via the manufacturer's own data logging program, which store the results in a 'txt' file format. In these experiments, the measurement speed of the LCR meter had been set at the "slow" setting of $0.896 \mathrm{~s}$, and the averages of 7 measurements were continuously recorded. It is possible to set a higher measurement frequency on the LCR meter, but this reduces the measurement accuracy.

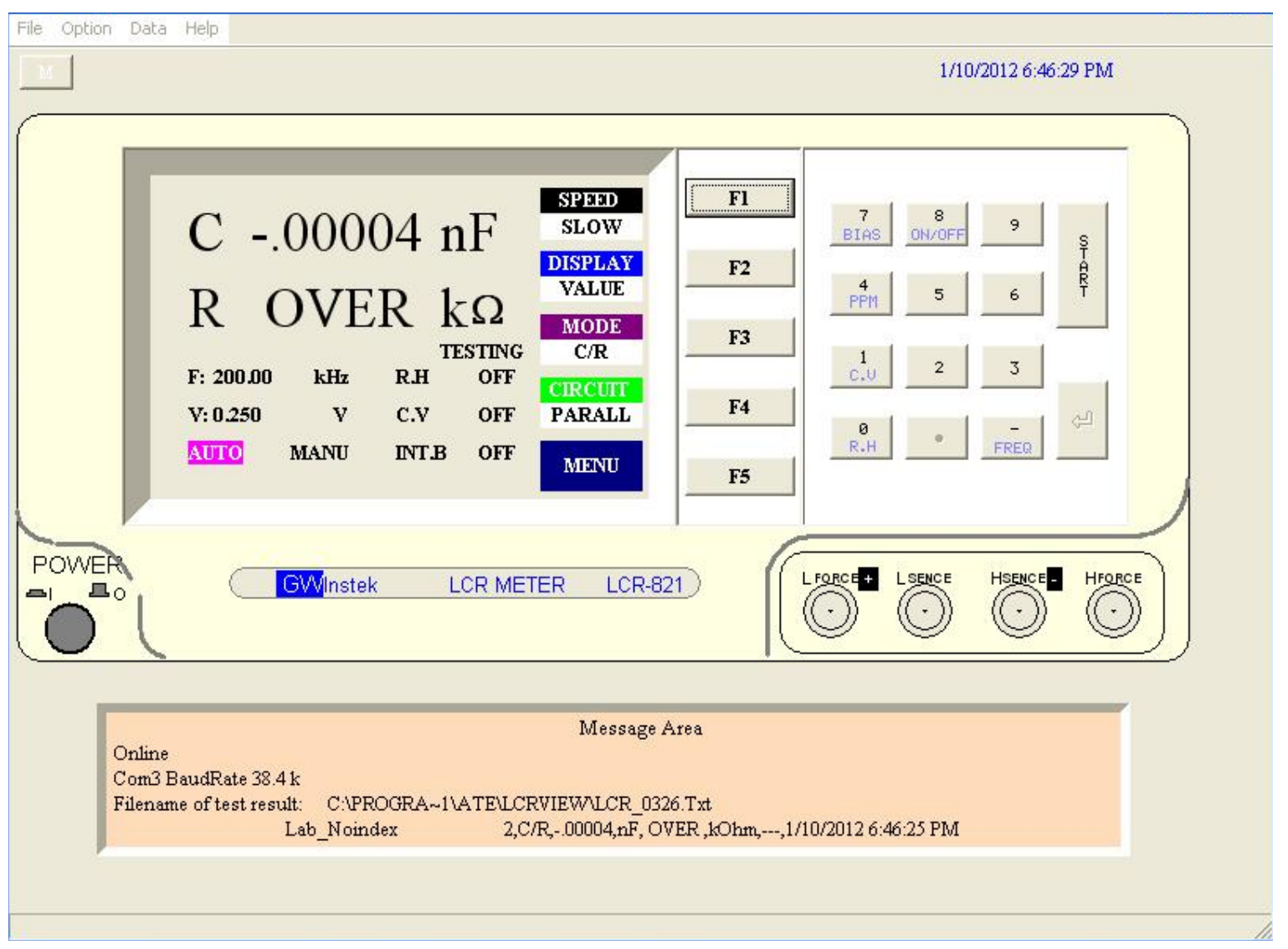

Fig. 4.7: GW-Instek LCR meter program user interface

\section{Wear Measurement}

Wear was continuously monitored by measurement of the wear track width via a Dino-lite AM413ZT digital microscope fitted above the disc. The digital microscope was set to capture a video of the wear track at 30-min frame intervals for the duration of the experiment. After 
each test, the ball and wear disc would be removed from the setup, where their wear scar diameter and wear track width respectively would be measured using an optical microscope. The wear track width at each video frame was then measured by scaling the pixels with reference to the final wear track width. The magnification of the digital microscope was set at $36 \times$ and the precision of wear measurement at this magnification is about $10 \mu \mathrm{m}$ per pixel.

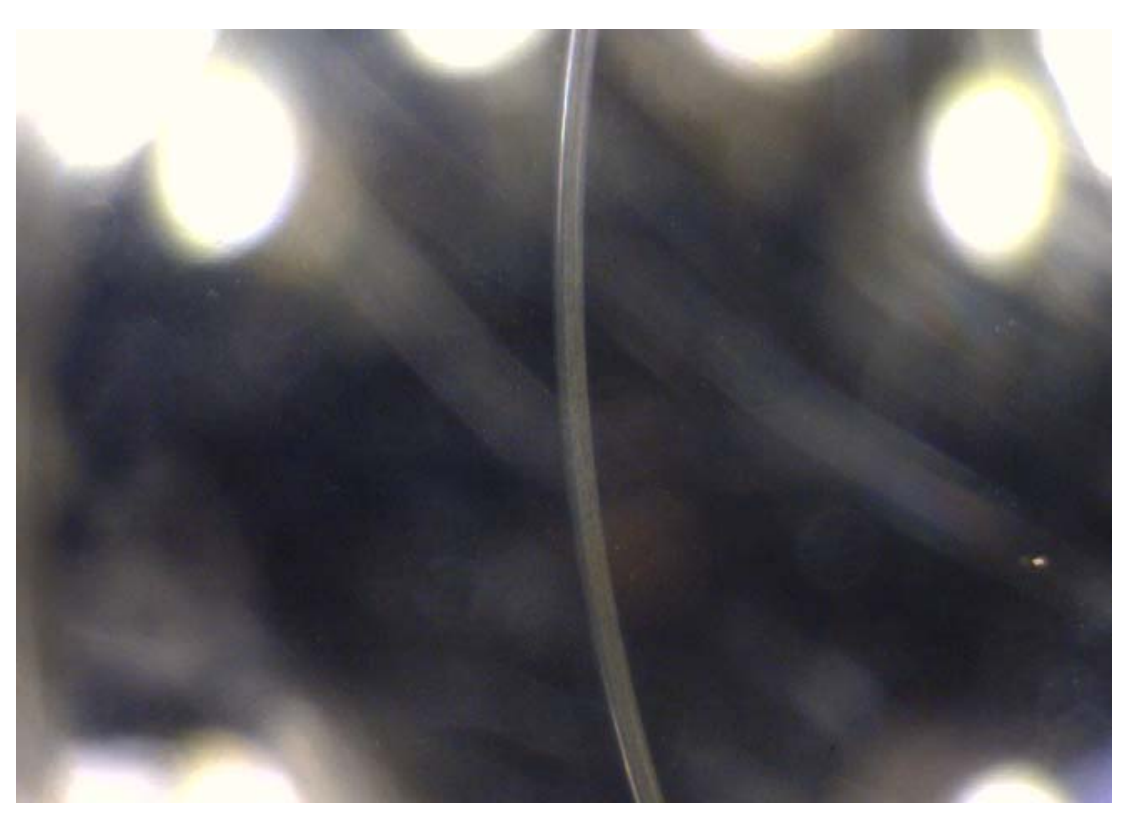

Fig. 4.8: Frame capture using DinoLite camera used to monitor the wear track. (Magnification: 36X)

\subsection{PILOT TEST USING SUNFLOWER OIL}

\subsubsection{Test Lubricant}

The test lubricant selected for the pilot test is a refined, bleached and deodorized (RBD) polyunsaturated sunflower oil obtained from Cargill. Gas Chromatography Mass Spectrometer (GCMS) revealed that the sunflower oil has a high level of polyunsaturated fatty acid content, with the major constituents as follows: palmitic acid $-9.5 \%$; stearic acid $-4.6 \%$; oleic acid $30 \%$; linoleic acid $-46.1 \%$. Its properties at $40{ }^{\circ} \mathrm{C}$ and ambient pressure, are as follows: 
density, $\varrho-0.901 \mathrm{~g} / \mathrm{cm}^{3}$; refractive index at $23{ }^{\circ} \mathrm{C}, n-1.476$; dynamic viscosity, $\eta-30.3 \mathrm{mPas}$; dielectric constant, $\varepsilon_{b}-2.575$; dielectric constant at $0.69 \mathrm{GPa}, \varepsilon_{r}-2.647$.

The refractive index was measured under ambient conditions using an Abbe Refractometer and the dynamic viscosity at $40^{\circ} \mathrm{C}$ was determined using the Brooksfield Cone/Plate Digital Viscometer. Measurement of the dielectric constant under ambient pressure was made using the same capacitance cell developed and is described in detail in the following section. The dielectric constant at the contact pressure of $0.69 \mathrm{GPa}$ is predicted using the Onsager equation and the modified Tait-Tammann equation (refer to Section 3.3 for calculation method).

\subsubsection{Measuring the Dielectric Constant, $\varepsilon$}

To convert capacitance values to film thickness values, it was necessary to know the dielectric constant of the lubricant at the contact pressure. As mentioned in Chapter 3, empirical methods can be employed, but the density and dielectric constant of the fluid under ambient pressures would be required. This can be measured using the same capacitance cell as shown in Fig. 4.3. Measurement of the dielectric constant can be made by utilizing the following relationship: -

$\varepsilon_{b}=\frac{C_{b}}{C_{\text {air }}} \varepsilon_{\text {air }}$

Where $\varepsilon_{b}$ and $C_{b}$ is the dielectric constant and capacitance measured the when the cell is filled with the fluid, $\varepsilon_{\text {air }}$ is the dielectric constant of air under atmospheric pressure (taken as 1.00059) and $C_{\text {air }}$ is the capacitance measured when cell is empty.

Firstly, the guard ring of the capacitance probe was re-wired as the negative electrode, and the bearing ball was replaced with a spherical electrical insulator, such that the flat surface of the guard separates the flat disc by a parallel gap of approximately $1 \mathrm{~mm}$. The capacitance of 
this air gap, $C_{a i r}$ is then measured. The reason why the guard ring has been used as the negative electrode instead of the ball, is because the guard has a larger surface area compared to the ball, and geometrically generates a greater capacitance when placed close to the positively charged disc. The larger capacitance increases the measurement accuracy of the LCR meter.

The test lubricant is then slowly poured into the cell until the gap is fully flooded and the capacitance of the test lubricant, $C_{b}$ is then measured. It is not critical that the actual gap distance is kept exactly the same when the measurement is made for different lubricants, as long as 1) the gap remains unchanged for that particular fluid before the fluid is poured into the cell and 2) the capacitance of this air gap is measurable by the LCR meter at the same manufacturer's rated accuracy. Measurements were made at the test excitation frequency of $200 \mathrm{kHz}$ and $0.25 \mathrm{~V}$, in the parallel mode. Parasitic capacitances from the connecting leads are automatically offset from the measurement by the LCR meter's compensation procedure. Capacitance readings are recorded continuously throughout this entire procedure until thermal equilibrium is reached and there is little change in the capacitance readings. The dielectric constant of the bulk lubricant at the test temperature is then calculated based on eqn. 7 above. Once the dielectric constant of lubricant at the test temperature and atmospheric pressure is known, empirical method described in Chapter 3 can be employed to determine the dielectric constant at the average Hertzian contact pressure.

\subsubsection{Experimental Details}

In this pilot test, the rotational speed was set at $10 \mathrm{rpm}$, corresponding to a sliding speed of $0.02 \mathrm{~m} / \mathrm{s}$. The theoretical central film thickness based on EHL theory would be less than 5 $\mathrm{nm}$ at this test temperature. This is less than the combined surface roughness of $17 \mathrm{~nm}$, so the lubrication regime here would be predominantly boundary lubrication. 
Before each experiment, the wear disc was ground with SiC paper of grit 120, 240, 500 and 1200 , and then finally polished to a mirror finish of $13 \mathrm{~nm} \mathrm{R}_{\mathrm{a}}$ with diamond suspension at 6, 3 and $1 \mu \mathrm{m}$. The disc was then washed once with cold tap water and then with ethanol after polishing, and wiped dry with soft tissue paper. Prior to installation, both the disc and a new ball were ultrasonically bathed in acetone for $15 \mathrm{~min}$, briefly washed again with ethanol, hexane and acetone, and finally blown dry with hot air. The ball was then secured in the capacitance probe by a nylon tipped grub screw, and the resistance was checked with a handheld multimeter to ensure good connection with the electrode seat.

After both the disc and bearing ball had been securely mounted, the LCR meter was then compensated to offset the parasitic capacitance and impedance of the leads during measurement. This was done by an 'open' and 'short' circuit test, as required by the LCR meter's zeroing procedure. During the 'short' circuit test, the capacitance probe was loaded with the same load used in the test. This ensured that the resistance was set to zero at the initial Hertzian contact.

The test lubricant was then slowly poured onto the wear disc and allowed to fully cover the latter, until the gap between the electrode guard and the disc was entirely flooded in the test lubricant. The whole cell, including the test lubricant was then gradually brought to the test temperature $\left(40^{\circ} \mathrm{C}\right.$ in these experiments), and then allowed to sit for $30 \mathrm{~min}$ to ensure that thermal equilibrium was reached. ${ }^{1}$ Finally, the bearing ball was then loaded with the dead weight, giving a test load of $0.7 \mathrm{~kg}$ at the contact. The mean Hertzian contact pressure and contact radius at this test load was $0.69 \mathrm{GPa}$ and $56.3 \mu \mathrm{m}$ respectively.

\footnotetext{
${ }^{1}$ Inconsistent results in the friction and film thickness trends were obtained if both the sliding ball and the disc were not allowed to be immersed in the test oil for at least 30 min before commencement of the test.
} 


\subsubsection{Experimental Results and Discussion}

Contact between asperities or the entrainment of large metallic wear particles in the contact zone usually results in a significant decrease in resistance and a negative capacitance reading on the LCR meter. This usually occurs for very thin films. In smoothing the signal, the negative capacitance readings were changed to infinity (mathematically corresponding to a zero film thickness). Ten consecutively recorded readings were then averaged, and the infinity values were rejected. This effectively retained capacitance readings that were measurable for at least a minute.

Fig. 4.9 shows the smoothed capacitance and resistance traces acquired simultaneously by the LCR meter. During the test, capacitance values reached as low as $36 \mathrm{pF}$ and resistance reached as high as $150 \mathrm{k} \Omega$. The friction and film thickness plots of sunflower oil are shown in Fig. 4.10. The most notable observation is the appreciable growth in the film thickness, which is mirrored by a decrease in friction. Initially, the boundary film thickness varied between 3-5 nm. After approximately $1 \mathrm{hr}$, there appeared to be a transition, where the film thickness appeared to steadily increase over time. The frictional trace was also observed to be much smoother after this transition. It is uncertain whether this is due to a completion of the running-in phase or a tribochemical reaction. The film thickness continued to increase until a maximum of about $8 \mathrm{~nm}$ at approximately $450 \mathrm{~min}$, after which it appeared to decline rapidly. After about $560 \mathrm{~min}$, the film seemed to have collapsed almost completely, though it is uncertain at this point why this occurred. 

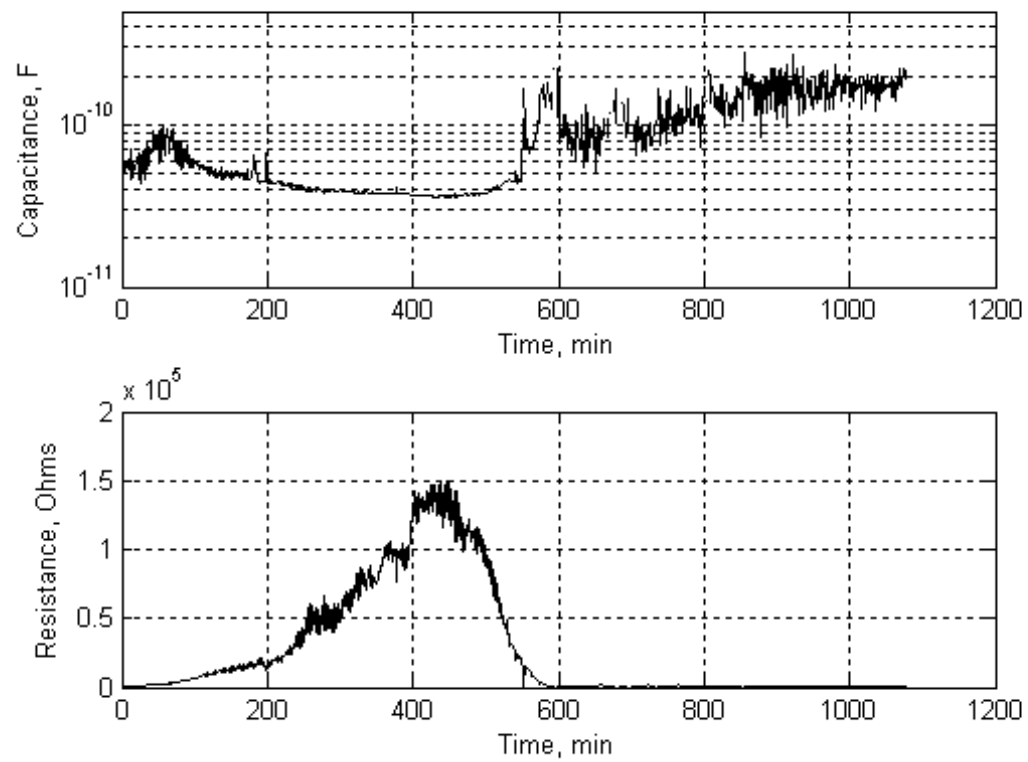

Fig. 4.9: (a) Capacitance trace versus sliding time; (b) Resistance trace versus sliding time

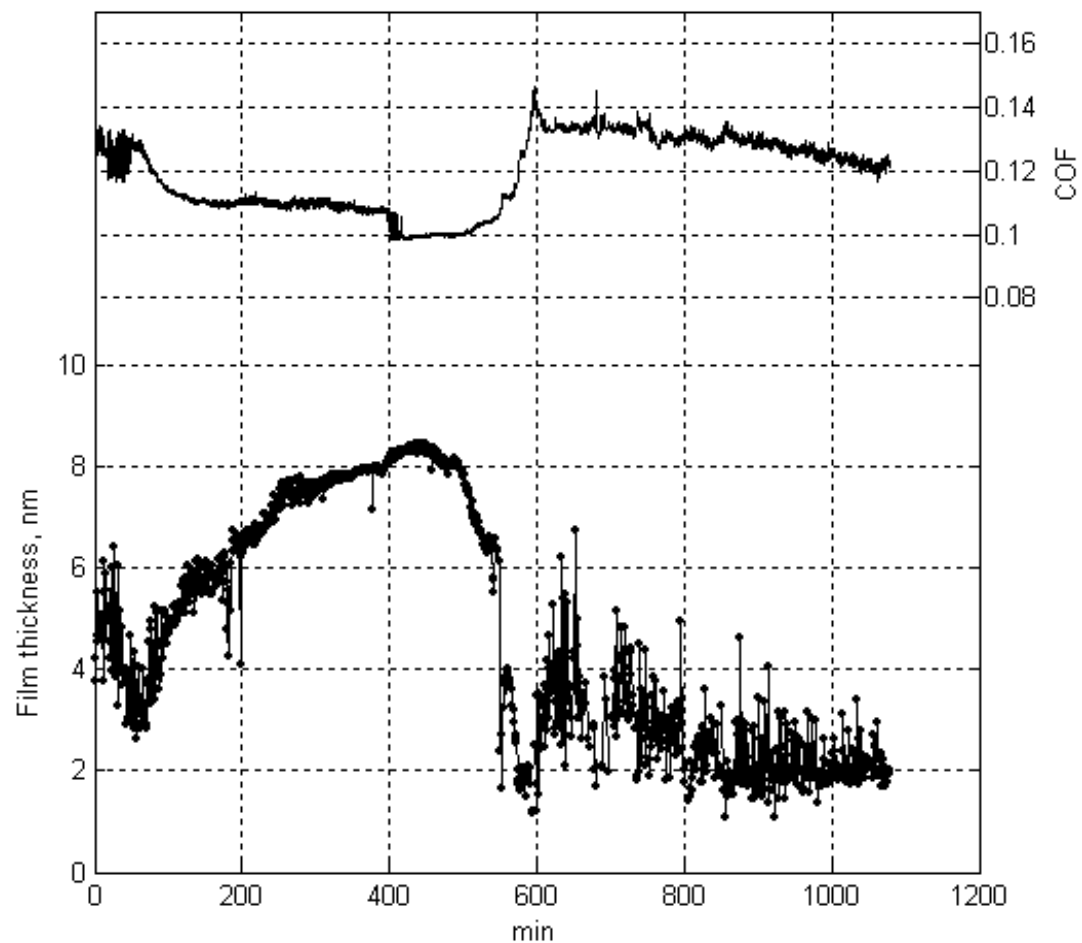

Fig. 4.10: Film thickness and COF versus sliding time plot 
Repeat tests showed some variation in the measured peak film thickness from $7 \mathrm{~nm}$ to $12 \mathrm{~nm}$ and in the film breakdown time from $300 \mathrm{~min}$ to $600 \mathrm{~min}$, presumably due to high sensitivity to test conditions. However, a distinct trend was observed in all the friction and film thickness traces: a short running in period, followed by a gradual growth in film thickness accompanied by a decrease in friction, and finally a rapid decline in the film thickness, accompanied by a return to high frictional values and a high wear rate.

Wear track width measurements show that the sunflower oil was effective in preventing wear when the film was present and growing in thickness (see Fig. 4.11). Wear increased rapidly once this film had collapsed. The initial wear track width is in close agreement with the theoretical Hertzian contact diameter. Examination of the final ball wear scar and wear track (see Fig. 4.12) under the optical microscope revealed a reddish brown topography that resembled that of corrosion products on the wear track, while no sign of this coloration was observed on the ball wear scar. Further studies would be required to determine the chemical nature of this substance.

It is postulated that the observed film growth was due to the polymerization of fatty acid chains. The concept of the formation of polymeric films reducing friction and wear is not new. It was first introduced by Furey [1] involving the use of a $\mathrm{C}_{36}$ dimer acid/ethylene glycol blend. Sunflower oil contains a large number of double bonds, $\mathrm{C}=\mathrm{C}$ due to the high proportion of polyunsaturated fatty acids in its triglyceride structure. The double bonds are largely responsible for the oil's poor oxidative stability, but are also essential to the polymerization process. The polymerization process occurs by the reaction of the carboxyl group of one fatty acid with the double bond of another. Both high temperatures and the presence of iron catalysts (which would always be present to some extent in the form of micro wear particles) have been known to favour this process [2]. 


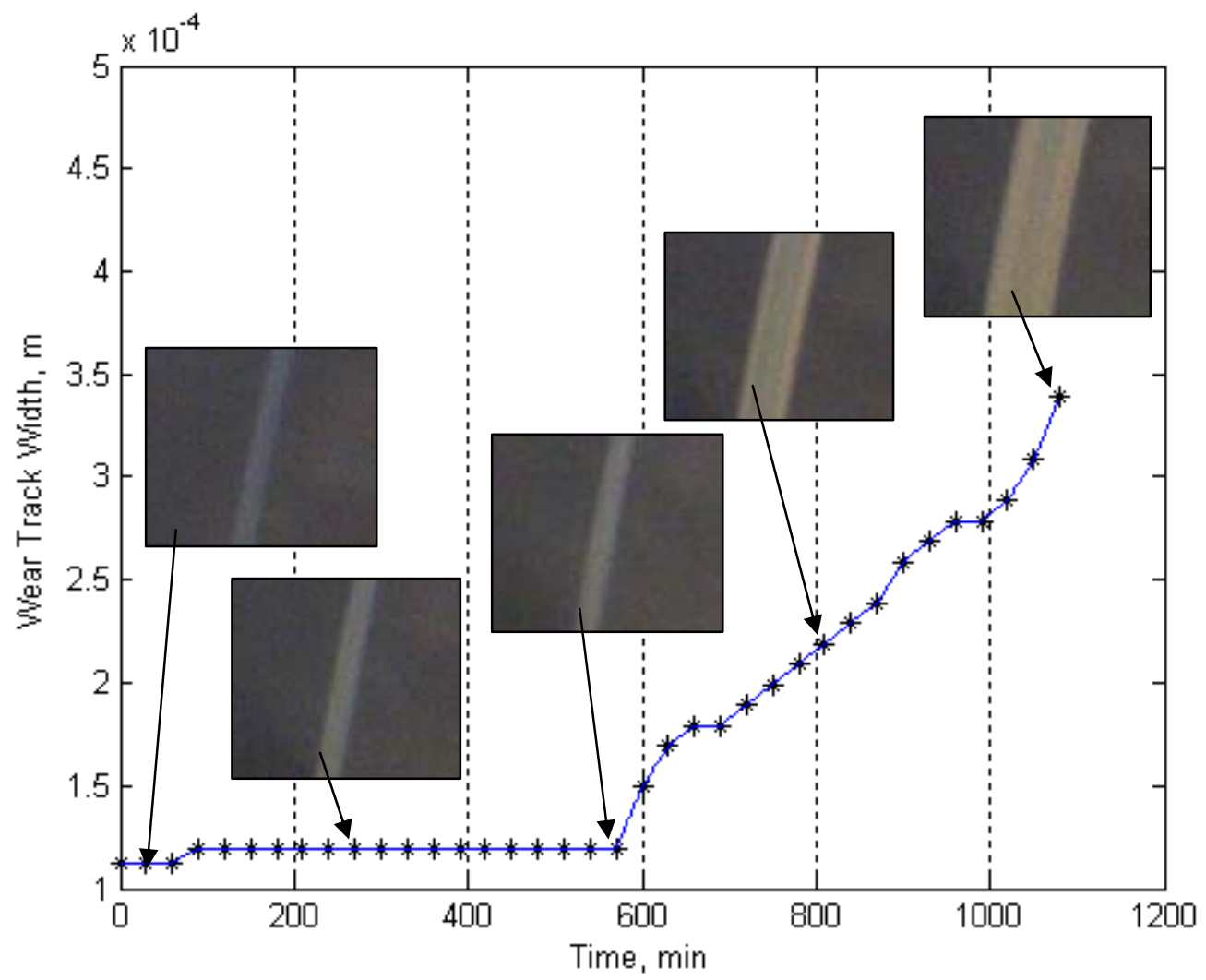

Figure 4.11: Wear track widths versus sliding time measured using images captured at 30 min intervals from a digital microscope.

a)

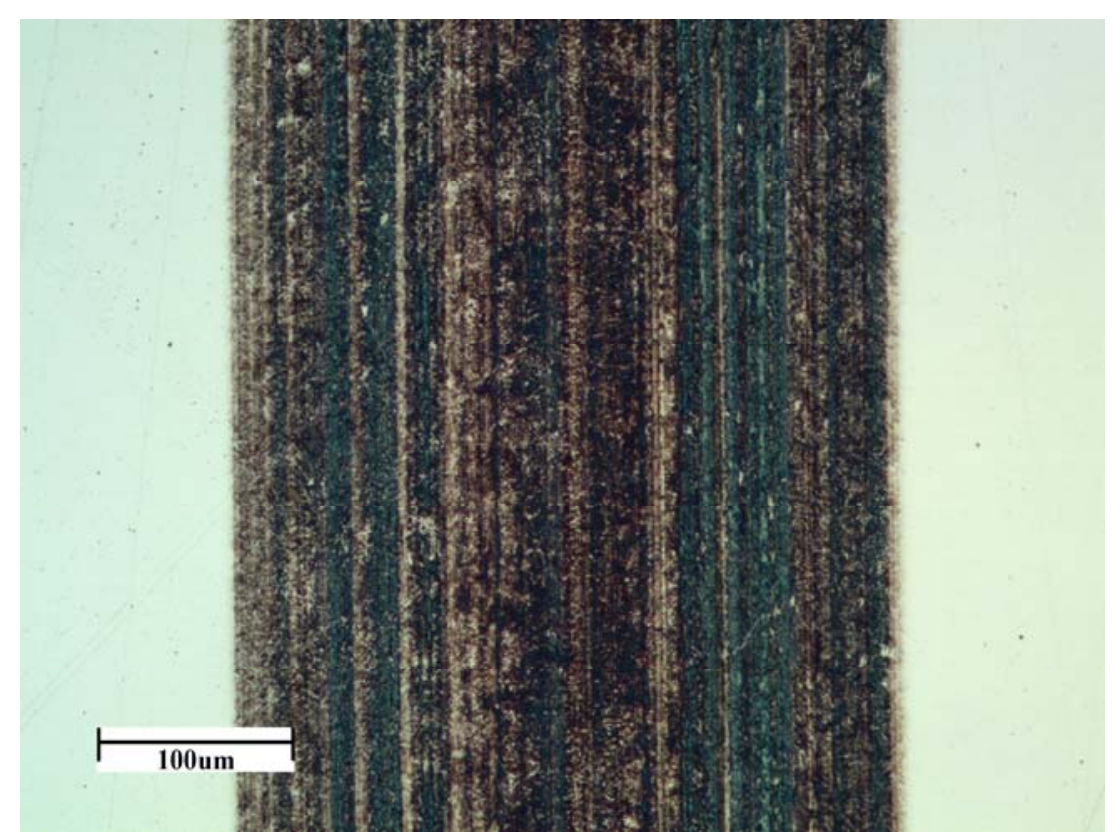

Cont'd 
b)

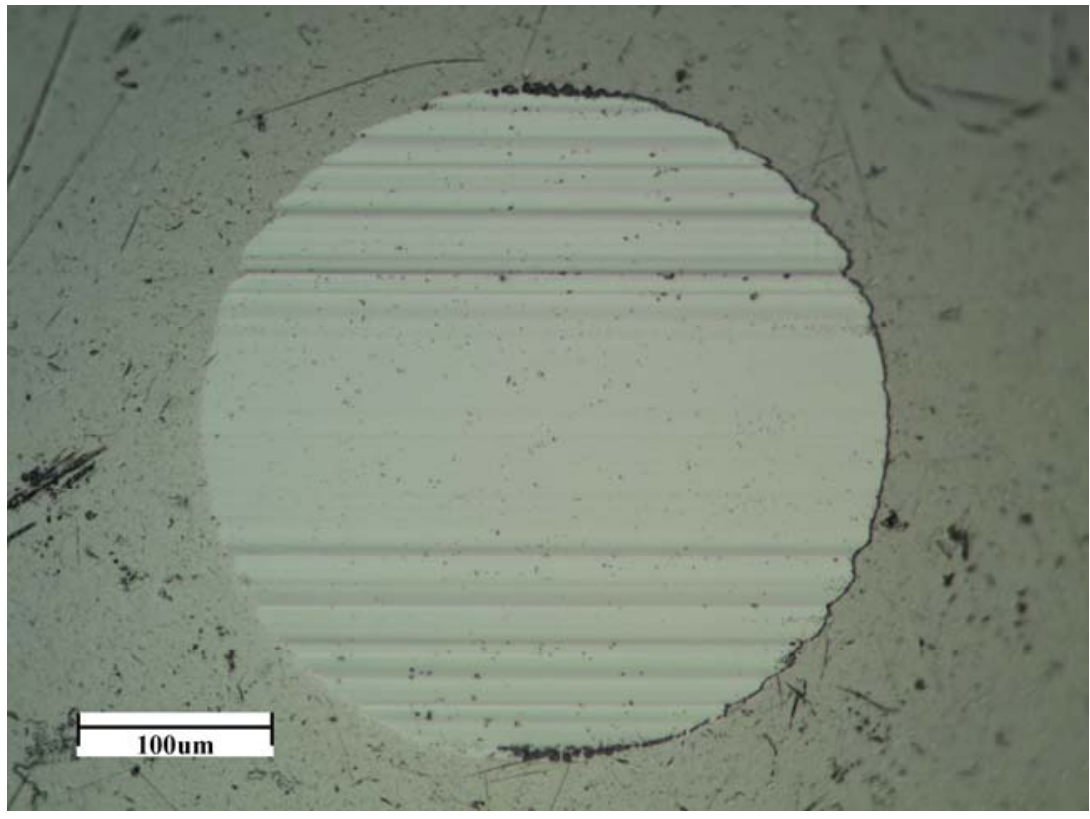

Figure 4.12: Optical micrographs of a) wear track on the disc; and b) ball wear scar

\subsection{DISCUSSION ON THE ACCURACY AND LIMITATIONS OF THE METHOD}

One major concern regarding the accuracy of film thickness measurement via capacitance methods has been the uncertainty of the dielectric constant. The dielectric constant is not only influenced by pressure and temperature of the contact, but is also very sensitive to the presence of water. While it is possible to make reasonable assumptions on what the local pressures and average contact temperature may be, the greatest uncertainty arises from the fact that, during sliding, a change in the molecular structure of the boundary film due to tribochemical reactions would likely affect its dielectric constant. An increasing dielectric constant due to oxidation or polymerization will, in effect, shift the capacitance versus film thickness curve up, as shown in Fig. 4.13. The dielectric constant of a vegetable oil blend of cottonseed and sunflower oil was reported to have increased by as much as 0.5 during the accelerated oxidation process [3]. It should be noted, however, that the impact of a change in the dielectric constant on the accuracy decreases for a decreasing film thickness. For instance, 
an increase of the dielectric constant of sunflower oil from 2.65 to 3.15 would underestimate the actual film thickness of $73 \mathrm{~nm}$ by $10 \mathrm{~nm}$; while an actual film thickness of $3.3 \mathrm{~nm}$ would be underestimated by only $0.5 \mathrm{~nm}$.

Another limitation of the method is that an assumption on the species adsorbed on the surface must be made for the dielectric constant to be determined. This does pose a problem for pure oils, such as refined sunflower oil, since the adsorbed boundary film is of the same chemical makeup as the bulk fluid. However, in the case of base oils blended or doped with one or more additives, it must be assumed that the dielectric constant of the boundary film will be that of the more polar constituent. The same limitation also applies for the optical interferometry method, since the refractive index of the film must be known in order to calculate the film thickness.

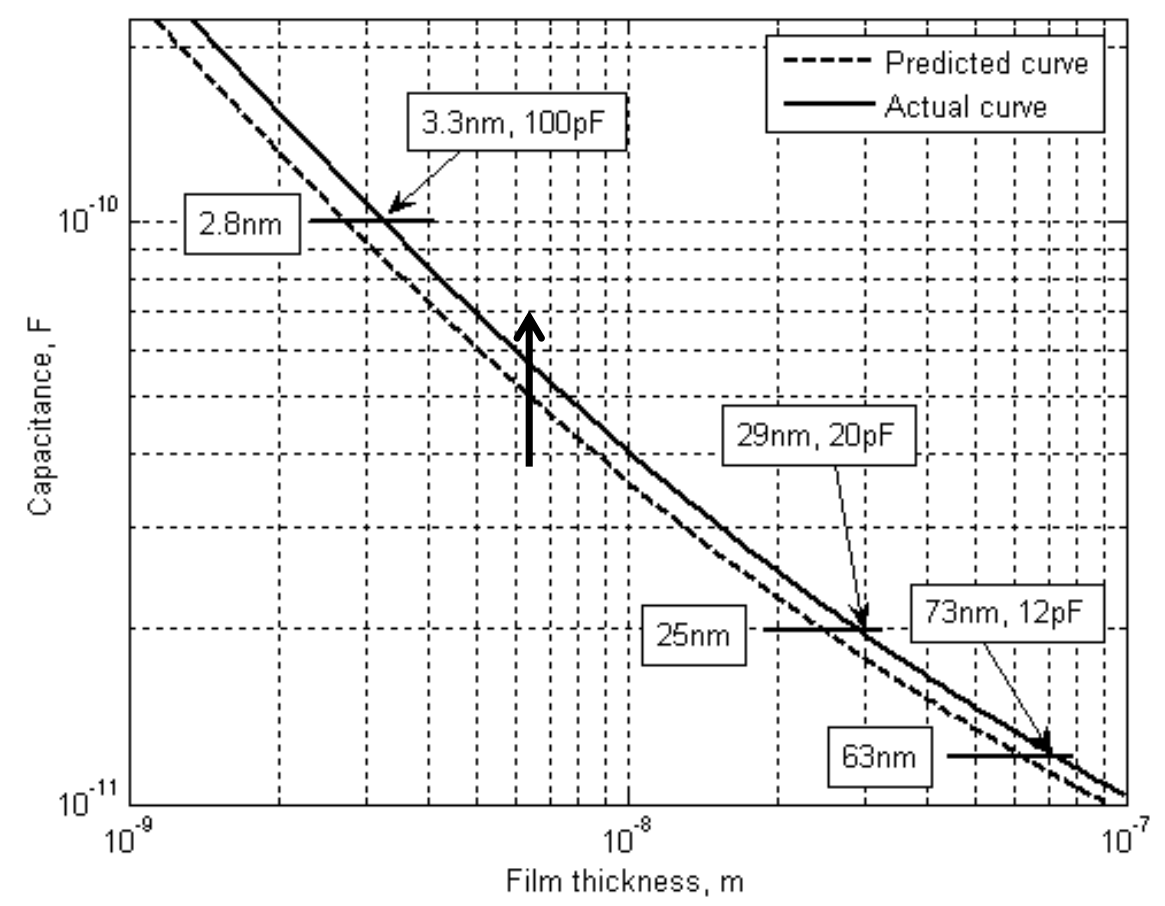

Fig. 4.13: Chart showing the actual film thickness curve versus the predicted film thickness curve for an increase in the dielectric constant of the boundary film from 2.65 to 3.15 . 
In the present model, it is assumed that, as the apparent area of contact increases due to wear, the real area of contact remains the same during sliding. If this assumption is incorrect, and the real area of contact grows significantly during sliding, then the measurement method will produce erroneous results. Conservatively, the accuracy of the method is maintained as long as wear is not significantly excessive, as in the present case with sunflower oil, when a relatively thick film is present.

There is also a concern that the generation of capacitance, would polarize the boundary film formed, or electrochemically interfere with the tribochemical process. For this reason, a low electrical potential of $0.25 \mathrm{~V}$ has been chosen for the impedance measurement. Lower potentials can be selected but this reduces the accuracy of the LCR meter. To address this concern, the test was repeated under the same conditions without connecting the capacitance sensor to the LCR meter, and results revealed the same colouration on the wear track, together with similar friction and wear trace characteristics, indicating that the electrical potential chosen for this work had a negligible effect on the tribochemical process.

While the capacitance method inherently possesses some flaws and limitations, it is the most direct method to measure the boundary film thickness between sliding metallic surfaces over long periods of time, and to monitor its changes with respect to wear and friction - a task otherwise not possible with the optical interferometry method.

\subsection{CONCLUSIONS}

A new method for the simultaneous measurement of friction, wear and boundary film thickness has been developed. The motivation behind this development was to allow for the studies of boundary film growth under practical tribological conditions. Boundary films formed by sunflower oil have been shown to grow to thicknesses greater than the expected heights of their adsorbed monolayers. It is postulated that this growth was due to the 
polymerization of unsaturated fatty acid chains. It would thus be of interest to compare the results obtained herein for sunflower oil, with other plant-derived oils with different degrees of unsaturation.

\section{REFERENCES}

1. Furey, M.J.: The formation of polymeric films directly on rubbing surfaces to reduce wear. Wear 26, 369-392 (1973)

2. Chaiyasit, W., Elias, R.J., Mcclements, D.J., Decker, E.A.: Role of physical structures in bulk oils on lipid oxidation. Crit. Rev. Food Sci. Nutr. 47, 299-317 (2007)

3. El-Shami, S.M., Zaki Selim, I.M., El-Anwar, Hassan El-Mallah, M.: Dielectric properties for monitoring the quality of heated oils. J. Am. Chem. Soc. 69(9), 872-875 (1992) 


\section{The Growth of Thin Lubricating Films of Plant Oils}

\subsection{INTRODUCTION}

Following the development of new tribometer and pilot test using refined sunflower oil described in Chapter 4, the work was extended to twelve other plant oils. A more detailed study was, however, conducted in this work. Other physical properties, such as density, viscosity, dielectric constant and refractive index were measured. Their fatty acid compositions and free fatty acid content were also analyzed. The purpose of this work is to study the film growth behaviours of a range of refined and unrefined plant oils with diverse fatty acid compositions, and to determine if there is a relationship between the oils' film growth behaviour and their chemical composition and physical properties.

\subsection{EXPERIMENTAL DETAILS}

The apparatus used is the same as that described in Chapter 4. The materials and test conditions were kept the same as that described in detail earlier. Briefly, the pure sliding contact was made between a stationary $8 \mathrm{~mm}$ diameter ball and a rotating $60 \mathrm{~mm}$ diameter disk, both of which are made of 52100 steel. The ball used was a commercially available bearing ball with a rated hardness of $63-65$ HRB. The disc was heat-treated to a final hardness of $814 \pm 10$ $\mathrm{HV}$, and then polished to a mirror finish of $13 \pm 2 \mathrm{~nm} \mathrm{Ra}$, as measured using the AltiSurf optical profilometer. The oil to be tested was allowed to slowly warm to room temperature, 
except for palm olein, which needed to be heated in a water bath at $40{ }^{\circ} \mathrm{C}$ until it fully melted. Approximately $15 \mathrm{ml}$ of the oil was then poured into the lubricant cell so that the disc was fully immersed, and then left to sit for $1 \mathrm{hr}$ at the test temperature. The test duration was set at 18 hrs, equivalent to 10800 disk revolutions.

Friction was measured using a full-bridge strain gauge system on the cantilever load arm (spring constant $=2.9 \mathrm{~N} / \mathrm{mm}$ ) at a sampling rate of 1000 samples $/$ s. Capacitance and resistance measurements were simultaneously acquired using a GW Instek LCR-821 meter at the excitation frequency of $200 \mathrm{kHz}$ and sampling interval of $0.896 \mathrm{~s}$, where the bearing ball and disk served as the negative and positive electrode respectively. Both friction and impedance measurements were averaged over a period of $6 \mathrm{~s}$, which in this work, was the time taken for one complete disc revolution. A digital microscope positioned above the disk, captured video frames of the wear track at 30 min intervals during the entire test period. The capacitance data acquired was then used to calculate the film thickness using the calculation method described.

As before, the intent was to subject the plant oils to thin film lubrication, where thicknesses are in the order of a few molecular lengths of the triglyceride. The tribological conditions for these tests were thus selected as follows: sliding speed $-0.02 \mathrm{~m} / \mathrm{s}$, test temperature $-40^{\circ} \mathrm{C}$ and load $-7 \mathrm{~N}$. This load corresponds to an average contact pressure of approximately $0.7 \mathrm{GPa}$, with a Hertzian contact radius of $56.3 \mu \mathrm{m}$. The load was chosen such that contact pressures are below the bulk yield strength of the contacting materials. Based on published values of the pressure viscosity coefficients of plant oils at $40{ }^{\circ} \mathrm{C}\left(7-11 \mathrm{GPa}^{-1}\right)$ [1], the theoretical EHL film thickness would be approximately between 3 to $6 \mathrm{~nm}$ at the test speed. In this work, the polished steel disk and bearing ball have a combined surface roughness of approximately $24 \mathrm{~nm}$. The film thickness in the apparent area of contact would thus vary by an amount roughly equivalent to the combined surface roughness of the two 
surfaces. It should be noted that, although the capacitance method provides only an average value of the film thickness across the contact, it has the advantage of being inherently biased towards the minimum film thickness (at the asperities) when the film thicknesses are in the order of the surface roughness.

\subsection{TEST LUBRICANTS}

Three refined oils - canola oil, high oleic sunflower oil and palm olein were obtained from Cargill Inc. Nine cold pressed and unrefined oils - sunflower oil, high oleic sunflower oil, safflower oil, canola oil, sesame oil, extra virgin olive oil, macadamia oil, wheatgerm oil and flaxseed oil were purchased from Melrose Health. All the oils, except for flaxseed oil, have been stored at approximately $4{ }^{\circ} \mathrm{C}$ in the refrigerator. Due to its poor oxidation stability, flaxseed oil was stored in the freezer at approximately $-20{ }^{\circ} \mathrm{C}$. All the oils were tested as they were, without further modification or filtration.

Calculation of the film thickness from capacitance data requires that the dielectric constant at the average contact pressure and temperature to be known. This was calculated using empirical methods, as described in Chapter 4. The temperature at the contact was assumed to be the same as the bulk oil, since the sliding speed used in this work was low $(0.02$ $\mathrm{m} / \mathrm{s})$.

\subsubsection{Physical Properties}

The density was measured by weighing the mass of the oil in a $10 \mathrm{ml}$ graduated volumetric flask, which was previously calibrated with distilled water. The density at the average contact pressure of $0.7 \mathrm{GPa}$ was predicted using the Tait-Tamman equation, with constants $\mathrm{B}=110$ and $C=0.07325$, as described in ref. 12. The dynamic viscosities were measured using the Brooksfield Cone/Plate Digital Viscometer at shear rates of 45, 22.5 and $11.25 \mathrm{~s}^{-1}$ and then averaged. The refractive indices were measured using an Abbe Refractometer, as per AOAC 
Official Method 921.08 [2]. All measurements were made at the temperature of $40{ }^{\circ} \mathrm{C}$ under ambient pressures. The density at $0.7 \mathrm{GPa}$ and refractive index determined were later used to calculate the dielectric constant at the same pressure.

\subsubsection{Dielectric Constant}

Improvements have been made to the measurement procedure for the dielectric constant since the initial pilot test described in Chapter 3. The measurement procedure is essentially the same, except that the ball, instead of the guard ring, was used as the negative electrode. The reason for this is that the guard ring is less shielded from fringing capacitances and creates an error that is dependant on the permittivity of the medium being measured. [3]. Although this effectively reduces the precision of the measurement due to smaller measurable capacitances, it significantly reduces the measurement error due to fringing capacitances. This fringing capacitance is a characteristic of the capacitance cell and must be taken into account when measuring the dielectric constant of test lubricants. It follows that Eqn. 7 must therefore be modified to include a cell factor, $\mathrm{k}-$

$$
\varepsilon_{b}=\frac{C_{b}}{C_{a i r}} \cdot \varepsilon_{a i r} \cdot k
$$

This cell factor was determined by using liquids of known dielectric constants [4] at $40{ }^{\circ} \mathrm{C}$. Five liquids - cyclohexane, chlorobenzene, toluene, 1-octanol and glycerol, of which the first two are standard calibration liquids, were used. The capacitance of the calibration liquids were measured as described previously, at $40{ }^{\circ} \mathrm{C}$. The cell factor, $\mathrm{k}$ was then determined by averaging all the $\mathrm{k}$ values found for each calibration liquid (see Table 5.1). This is calculated to be 0.9328 . 
Table 5.1: Determination of the cell factor, $k$

\begin{tabular}{ccccc}
\hline Calibration Liquid & $C_{\text {air }}(\mathrm{pF})$ & $C_{b}(\mathrm{pF})$ & $\varepsilon_{\text {ref }}$ & $k=\frac{C_{\text {air }} \cdot \varepsilon_{\text {ref }}}{C_{b} \cdot \varepsilon_{\text {air }}}$ \\
\hline Glycerol & 0.631 & 26.719 & 39.55 & 0.933468 \\
$\begin{array}{c}\text { 1 Octanol } \\
\text { Chlorobenzene }\end{array}$ & 0.584 & 5.419 & 8.5278 & 0.91849 \\
Toulene & 0.650 & 3.775 & 5.378 & 0.925467 \\
Cyclohexane & 0.648 & 1.640 & 2.309 & 0.921648 \\
\hline
\end{tabular}

As before, once the dielectric constant at the ambient pressure is known, the Onsager equation was used to predict the dielectric constants at the contact pressure of $0.7 \mathrm{GPa}$.

The physical properties measured are listed in Table 5.2. These results are in agreement with those obtained from literature [5] in that oils with a higher degree of unsaturation are less viscous, and have higher densities, refractive indices and dielectric constants. The plots of the oils' refractive indices, dielectric constants, viscosities and degrees of unsaturation against their densities are shown in Fig. 5.1. A notable outlier for the viscositydensity relationship is wheatgerm oil. This is most likely due to the high level of insoluble matter present in the oil. 
Table 5.2: Physical properties. All properties are determined at $40^{\circ} \mathrm{C}$ and ambient pressure, unless stated otherwise.

\begin{tabular}{lccccc} 
Plant oil & $\begin{array}{c}\text { Density, } \rho \\
(\mathrm{g} / \mathrm{cm})\end{array}$ & $\begin{array}{c}\text { Dielectric } \\
\text { constant, } \varepsilon\end{array}$ & $\begin{array}{c}\text { Refractive } \\
\text { index, } n\end{array}$ & $\begin{array}{c}\text { Predicted } \\
\text { dielectric } \\
\text { constant, } \varepsilon \\
\text { at } 0.7 \mathrm{GPa}\end{array}$ & $\begin{array}{c}\text { Dynamic } \\
\text { viscosity, } \eta \\
(\mathrm{cP})\end{array}$ \\
\hline & Refined, bleached and deoderised oils & & \\
\hline Canola oil & 0.905 & 3.00 & 1.470 & 3.15 & 32.6 \\
High oleic sunflower oil & 0.900 & 2.93 & 1.466 & 3.07 & 36.39 \\
Palm olein & 0.901 & 3.00 & 1.462 & 3.17 & 35.8 \\
\hline & Unrefined, cold-pressed oils & & \\
\hline Sunflower oil & 0.909 & 3.05 & 1.472 & 3.21 & 27.78 \\
High oleic sunflower oil & 0.900 & 3.00 & 1.467 & 3.16 & 36.39 \\
Canola oil & 0.904 & 3.02 & 1.469 & 3.18 & 33.46 \\
Sesame oil & 0.904 & 3.01 & 1.469 & 3.16 & 32.23 \\
Safflower oil & 0.909 & 3.14 & 1.472 & 3.32 & 28.22 \\
Extra virgin olive oil & 0.899 & 2.98 & 1.465 & 3.14 & 36.8 \\
Macadamia oil & 0.901 & 2.96 & 1.464 & 3.12 & 35.62 \\
Flaxseed oil & 0.916 & 3.11 & 1.477 & 3.29 & 23.65 \\
Wheatgerm oil & 0.916 & 3.13 & 1.474 & 3.31 & 36.8 \\
\hline
\end{tabular}



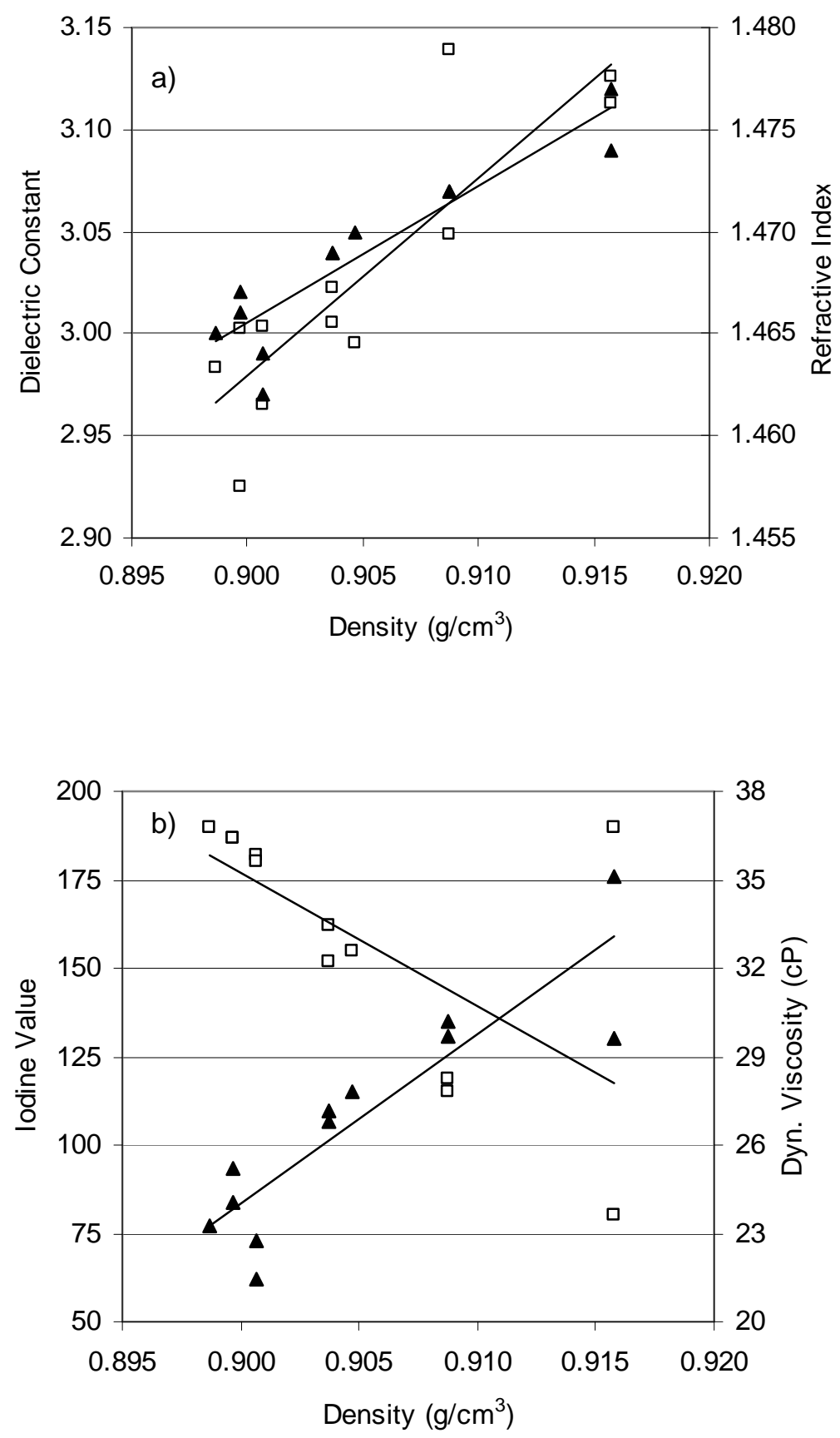

Fig. 5.1: a) Dielectric constant $\square$ and refractive index $\mathbf{\Lambda}$ vs. density; b) Iodine value $\boldsymbol{\Lambda}$ and dynamic viscosity $\square$ vs. density. 


\subsubsection{Fatty Acid Composition and Free Fatty Acids (FFAs)}

The fatty acid compositions were determined using gas chromatography mass spectroscopy (GCMS). $3 \mu \mathrm{l}$ of the oil sample was dissolved in $150 \mu \mathrm{l}$ of toluene and $300 \mu \mathrm{l}$ of methanol with $2 \% \mathrm{H}_{2} \mathrm{SO}_{4}$ was then added. The mixture was then refluxed at $80{ }^{\circ} \mathrm{C}$ for $2 \mathrm{hrs}$ to produce the fatty acid methyl esters (FAMEs). $300 \mu \mathrm{l}$ of $0.9 \% \mathrm{NaCl}$ solution and $300 \mu \mathrm{l}$ of hexane were then added. The mixture was briefly shaken and then centrifuged for $3 \mathrm{~min}$ at $13200 \mathrm{rpm}$. A Pastuer pipette was then used to remove the hexanic layer for analysis in GCMS. Nonadecanoic acid (C19:0) solution was used as an internal standard for the quantification of the FAMEs.

The GCMS was performed on a 7890A gas chromatography instrument, equipped with a 122-7032 DB-Wax capillary column (30 m x $0.25 \mathrm{~mm}$ I.D. x $0.25 \mu \mathrm{m}$ film thickness) and coupled with a 5975C inert XL mass selective detector (Agilent Technologies, Little Fall, NY, USA) and a MPS 2XL autosampler (Gerstel, Mülheim an der Ruhr, Germany). Helium was used as the carrier gas at a flow rate of $1.03 \mathrm{ml} / \mathrm{min} .1 \mu \mathrm{l}$ of the sample was injected applying the following conditions: injector temperature $-250{ }^{\circ} \mathrm{C}$; split ratio -1 :10; column temperature - from $50{ }^{\circ} \mathrm{C}$ (hold time $=1 \mathrm{~min}$ ), to $200{ }^{\circ} \mathrm{C}$ at $25{ }^{\circ} \mathrm{C} / \mathrm{min}$ and then to $230{ }^{\circ} \mathrm{C}$ (hold time $=18 \mathrm{~min}$ ) at $3{ }^{\circ} \mathrm{C} / \mathrm{min}$. The temperature of the transfer liner was set at $250{ }^{\circ} \mathrm{C}$. The mass spectrometer was operated at the ionization energy of $70 \mathrm{eV}$ and mass scanning was performed from 50 to $600 \mathrm{amu}$.

The amount of free fatty acids (FFAs) in the oils was determined by the AOAC Official Method 940.28 [2]. Iodine value was calculated using equation (2) in ref. 6, from the fatty acid compositions determined by GCMS. The iodine value provides a measure of the oil's degree of unsaturation.

The results of the GCMS and fatty acid determination are shown in Table 2. Wheatgerm oil has the highest level of FFAs among the oils tested, which is typical of most 
unrefined wheatgerm oils. Flaxseed oil differs from the other highly polyunsaturated oils in that a large proportion of its polyunsaturated fatty acids (PUFAs) is comprised of $\omega-3$ fatty acids (C18:3). It was also interesting to note that the highly monounsaturated macadamia oil has the highest concentration of long chain fatty acids with more than 20 carbon atoms, and also the second highest amount of FFAs among the oils tested.

Table 5.3: Fatty acid composition and free fatty acids. SFA = saturated fatty acids; MUFA = monounsaturated fatty acids; PUFA = polyunsaturated fatty acids. IV $=$ iodine value. FFA\% $=$ free fatty acids (as \% oleic acid)

\begin{tabular}{|c|c|c|c|c|c|c|c|c|c|c|c|c|}
\hline & \multicolumn{3}{|c|}{ Refined oils } & \multicolumn{9}{|c|}{ Unrefined cold pressed oils } \\
\hline & 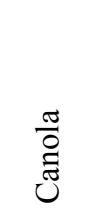 & 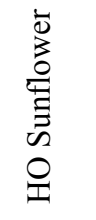 & $\begin{array}{l}\frac{\Xi}{0} \\
\Xi \\
\Xi \\
\sigma\end{array}$ & 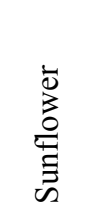 & 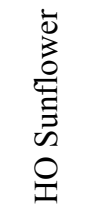 & 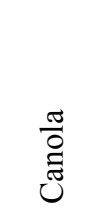 & $\begin{array}{c}\mathscr{E} \\
\mathbb{E} \\
\mathscr{D} \\
\mathscr{S}\end{array}$ & 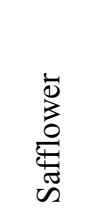 & 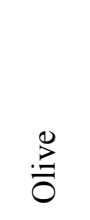 & 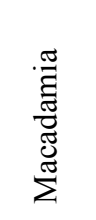 & $\begin{array}{l}\vec{\Xi} \\
\mathbb{U} \\
\tilde{X} \\
\stackrel{\Xi}{I}\end{array}$ & 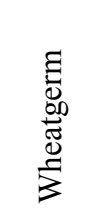 \\
\hline $\mathrm{C} 12$ & 0.00 & 0.00 & 0.29 & 0.00 & 0.00 & 0.00 & 0.00 & 0.00 & 0.00 & 0.05 & 0.00 & 0.00 \\
\hline $\mathrm{C} 14$ & 0.08 & 0.08 & 0.83 & 0.08 & 0.07 & 0.09 & 0.09 & 0.11 & 0.04 & 0.62 & 0.06 & 0.10 \\
\hline $\mathrm{C} 15$ & 0.02 & 0.02 & 0.04 & 0.02 & 0.02 & 0.02 & 0.01 & 0.02 & 0.01 & 0.02 & 0.03 & 0.05 \\
\hline $\mathrm{C} 16$ & 4.79 & 4.96 & 33.88 & 6.80 & 4.68 & 5.94 & 10.90 & 7.20 & 13.90 & 9.00 & 5.49 & 16.83 \\
\hline C16:1 & 0.17 & 0.10 & 0.16 & 0.04 & 0.11 & 0.24 & 0.13 & 0.06 & 0.73 & 16.18 & 0.05 & 0.17 \\
\hline $\mathrm{C} 16: 2$ & 0.02 & 0.00 & 0.00 & 0.00 & 0.00 & 0.00 & 0.00 & 0.00 & 0.00 & 0.00 & 0.00 & 0.02 \\
\hline $\mathrm{C} 17$ & 0.06 & 0.05 & 0.10 & 0.06 & 0.04 & 0.07 & 0.06 & 0.04 & 0.06 & 0.04 & 0.07 & 0.04 \\
\hline $\mathrm{C} 18$ & 2.84 & 4.23 & 5.54 & 5.49 & 3.93 & 3.19 & 5.89 & 3.70 & 3.65 & 4.25 & 5.53 & 1.38 \\
\hline C18:1 & 56.93 & 79.47 & 45.05 & 19.97 & 70.60 & 55.58 & 39.53 & 19.52 & 72.95 & 58.95 & 21.64 & 17.05 \\
\hline $\mathrm{C} 18: 2$ & 23.29 & 8.88 & 13.18 & 65.70 & 18.67 & 23.93 & 41.41 & 68.14 & 6.91 & 2.37 & 18.30 & 54.38 \\
\hline $\mathrm{C} 18: 3$ & 9.70 & 0.13 & 0.17 & 0.17 & 0.09 & 7.19 & 0.29 & 0.09 & 0.61 & 0.14 & 48.20 & 7.70 \\
\hline $\mathrm{C} 20$ & 0.53 & 0.33 & 0.40 & 0.30 & 0.30 & 0.75 & 0.76 & 0.40 & 0.57 & 3.32 & 0.17 & 0.12 \\
\hline $\mathrm{C} 20: 1$ & 1.01 & 0.25 & 0.18 & 0.15 & 0.23 & 1.57 & 0.31 & 0.22 & 0.33 & 3.19 & 0.15 & 1.57 \\
\hline $\mathrm{C} 20: 2$ & 0.07 & 0.00 & 0.00 & 0.02 & 0.00 & 0.09 & 0.01 & 0.03 & 0.00 & 0.00 & 0.05 & 0.18 \\
\hline $\mathrm{C} 22$ & 0.26 & 1.10 & 0.10 & 0.92 & 0.86 & 0.52 & 0.38 & 0.32 & 0.18 & 1.03 & 0.17 & 0.11 \\
\hline $\mathrm{C} 22: 1$ & 0.09 & 0.00 & 0.00 & 0.00 & 0.00 & 0.64 & 0.00 & 0.00 & 0.00 & 0.36 & 0.00 & 0.21 \\
\hline $\mathrm{C} 24$ & 0.14 & 0.40 & 0.08 & 0.27 & 0.39 & 0.18 & 0.22 & 0.16 & 0.06 & 0.47 & 0.11 & 0.10 \\
\hline SFA $\%$ & 8.73 & 11.17 & 41.27 & 13.94 & 10.28 & 10.76 & 18.31 & 11.95 & 18.47 & 18.81 & 11.62 & 18.74 \\
\hline MUFA\% & 58.19 & 79.82 & 45.38 & 20.17 & 70.95 & 58.02 & 39.97 & 19.80 & 74.01 & 78.68 & 21.84 & 18.99 \\
\hline PUFA\% & 33.08 & 9.01 & 13.35 & 65.89 & 18.77 & 31.22 & 41.72 & 68.26 & 7.52 & 2.51 & 66.54 & 62.27 \\
\hline IV & 115.30 & 83.97 & 62.01 & 131.00 & 93.16 & 109.61 & 106.37 & 134.67 & 76.89 & 72.97 & 175.79 & 130.22 \\
\hline FFA \% & 0.05 & 0.04 & 0.06 & 0.44 & 0.19 & 0.13 & 0.47 & 0.48 & 0.58 & 1.47 & 1.46 & 3.54 \\
\hline
\end{tabular}




\subsection{EXPERIMENTAL RESULTS}

\subsubsection{Film thickness and Friction}

The friction and film thickness traces were averaged over three repeat experiments, as shown in Fig. 5.2. For comparison, the mean film thicknesses of the first and final hour of the tests are shown in the bar chart of Fig. 5.3. It is clear from these results that the film thicknesses of some oils do not remain constant when subjected to continuous sliding contact. Some films grew as much as 25 times in thickness (unrefined canola oil), while others remained roughly the same (flaxseed and olive oil), or grew slightly and then collapsed during the test period (safflower oil). It would also appear that there is a maximum thickness that the films would grow to and this maximum thickness is unique to each oil. While there is a distinct relationship between the properties and the degree of unsaturation of the oils, the relationship between the latter and the film growth is less clear.

The coefficient of friction (COF) for the oils tested is in the range of 0.08 to 0.12 . At the start of sliding, the COF generally could be as high as 0.14 , but quickly decreased to a stable value with sliding time. With the exception of palm olein, the initial film thicknesses of the other oils tested are in the range of 4-10 nm. It is uncertain why palm olein formed a significantly thicker film than the other oils during the initial stages, but we believe that this might be due to the fact that the test temperature of $40{ }^{\circ} \mathrm{C}$ was relatively near its solidification temperature range. Since surface boundaries are the preferred initiation sites for crystallization, some of the more saturated triglycerides that are physically adsorbed could have formed crystallites on the surfaces during the 1-hr pre-test immersion period.

Highly polyunsaturated oils such as flaxseed and safflower oils generated very thin films $(<10 \mathrm{~nm})$, with the latter collapsing after $400 \mathrm{~min}$ of sliding. The film growth and collapse observed in safflower oil was similar to that observed in the pilot study (see Chapter 4) 
conducted using refined sunflower oil. Interestingly, although the fatty acid composition of unrefined sunflower oil is very similar to that of safflower oil, the former grew steadily to a thickness of approximately 40nm, without any sign of film breakdown. For wheatgerm oil, the film thickness increased quickly to approximately $30 \mathrm{~nm}$ within 3-4 hrs, but varied unstably between 15 to $30 \mathrm{~nm}$ thereafter.

From the results obtained, it appears that the high oleic oils, while being more stable, exhibited slower film growth. The film thickness of unrefined and refined high oleic sunflower oil grew and stabilized at approximately 12 and $15 \mathrm{~nm}$ respectively. The film thickness of palm olein peaked at 30-40nm after about 6 hours of sliding, but decreased and then stabilized at about $18 \mathrm{~nm}$. On the other hand, there was almost no change in the thickness of the film generated by olive oil throughout the entire test. Among the high oleic oils, macadamia oil exhibited the most stable film growth up to approximately $29 \mathrm{~nm}$ by the end of the test. This oil was observed to grow even up to $60 \mathrm{~nm}$ in one test. It is uncertain whether this was due to the increased presence of FFAs in the oil.

The most surprising results are those of two moderately unsaturated oils - unrefined sesame oil and unrefined canola oil, where their film thicknesses increased to 50-60 nm and 80-130 nm respectively. Among the oils tested, the fastest growth rate has been that of unrefined canola oil. Its growth to approximately $100 \mathrm{~nm}$ was completed in about 6-7 hours. It should be noted that refined canola oil, which has a similar fatty acid composition as its unrefined variant, but only half its acidity, did not exhibit the same film thickening behaviour. 

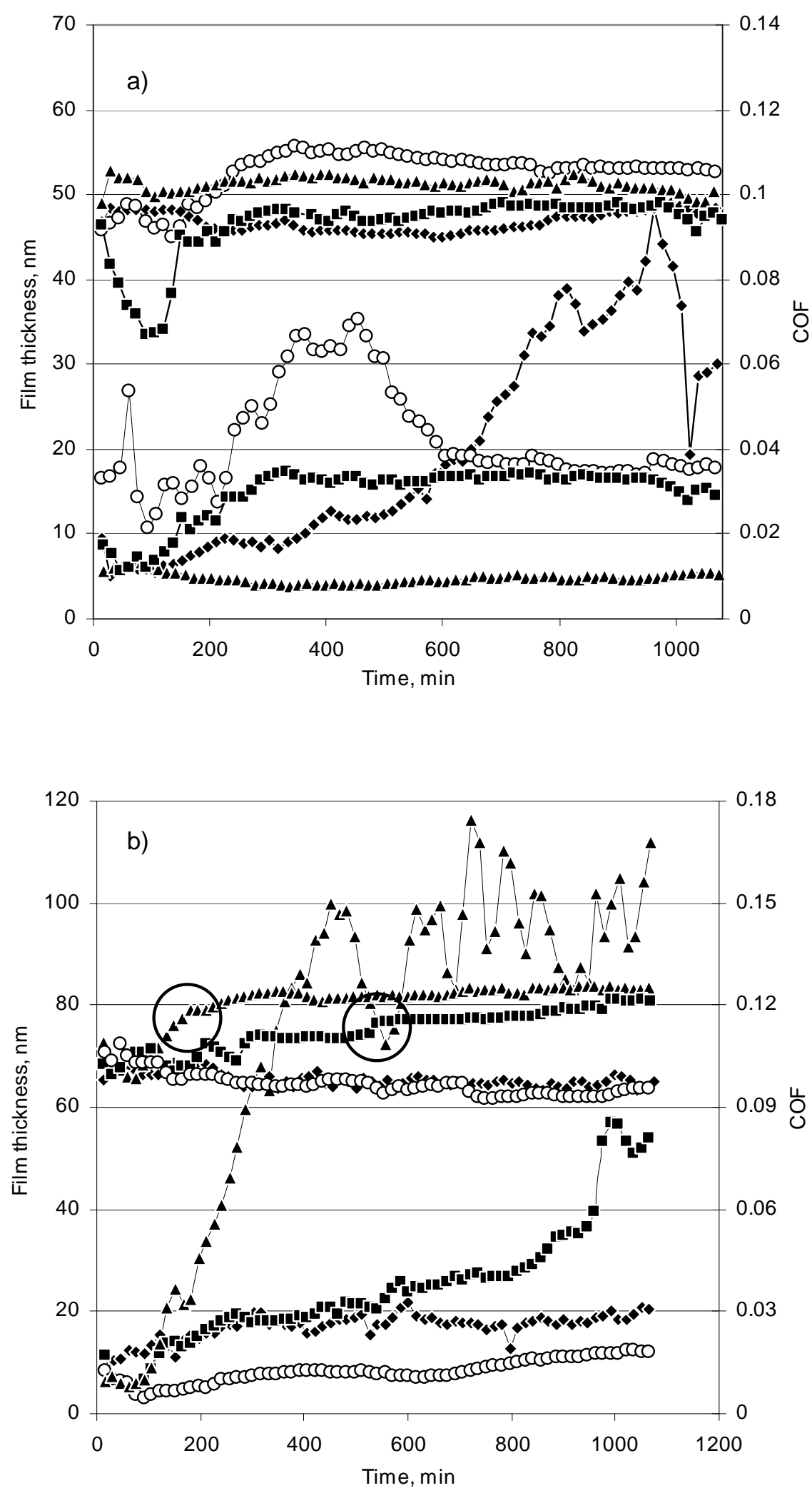

Cont'd 


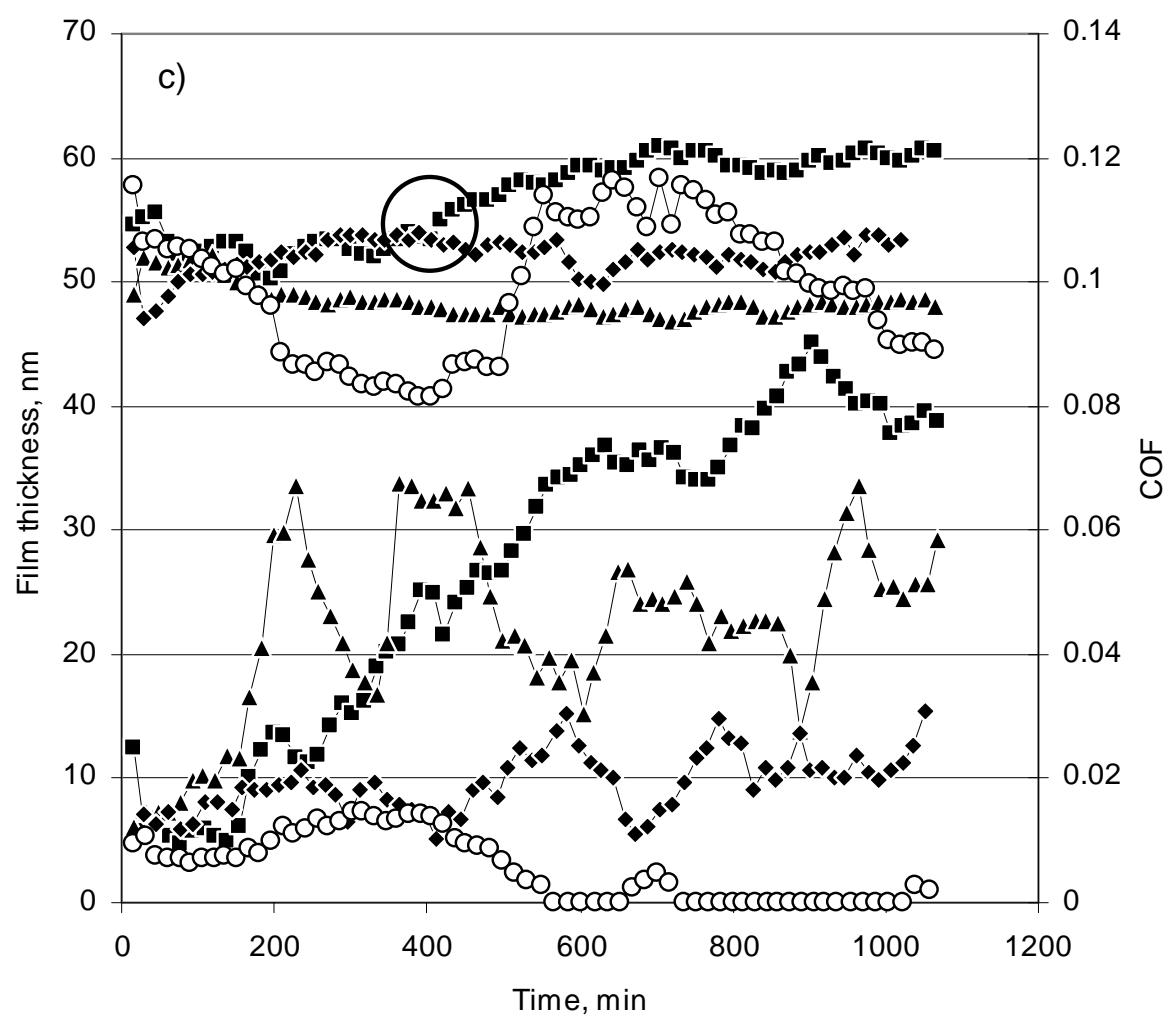

Fig. 5.2: Change of film thickness and friction with sliding time (upper traces and lower traces are for $\mathrm{COF}$ and film thickness respectively). a) Oils with $\mathrm{IV}<90$ : Extra virgin olive oil $\boldsymbol{\Delta}$; HO sunflower oil (refined) $\mathbf{\square}$; Macadamia oil $\diamond$; and Palm olein $O$; b) Oils with $90<I V<120$ : Canola oil (unrefined) $\mathbf{\Delta}$; Sesame oil $\mathbf{\square}$; Canola oil (refined) $\bullet$; and HO sunflower (unrefined) O. c) Oils with IV $>120$ :

Wheatgerm oil $\boldsymbol{\Delta}$; Sunflower oil $\mathbf{\square}$; Flaxseed oil $\bullet$; and Safflower oil $\bigcirc$. Circled areas denote points of significant increase in friction for unrefined canola oil, sesame oil and sunflower oil. 


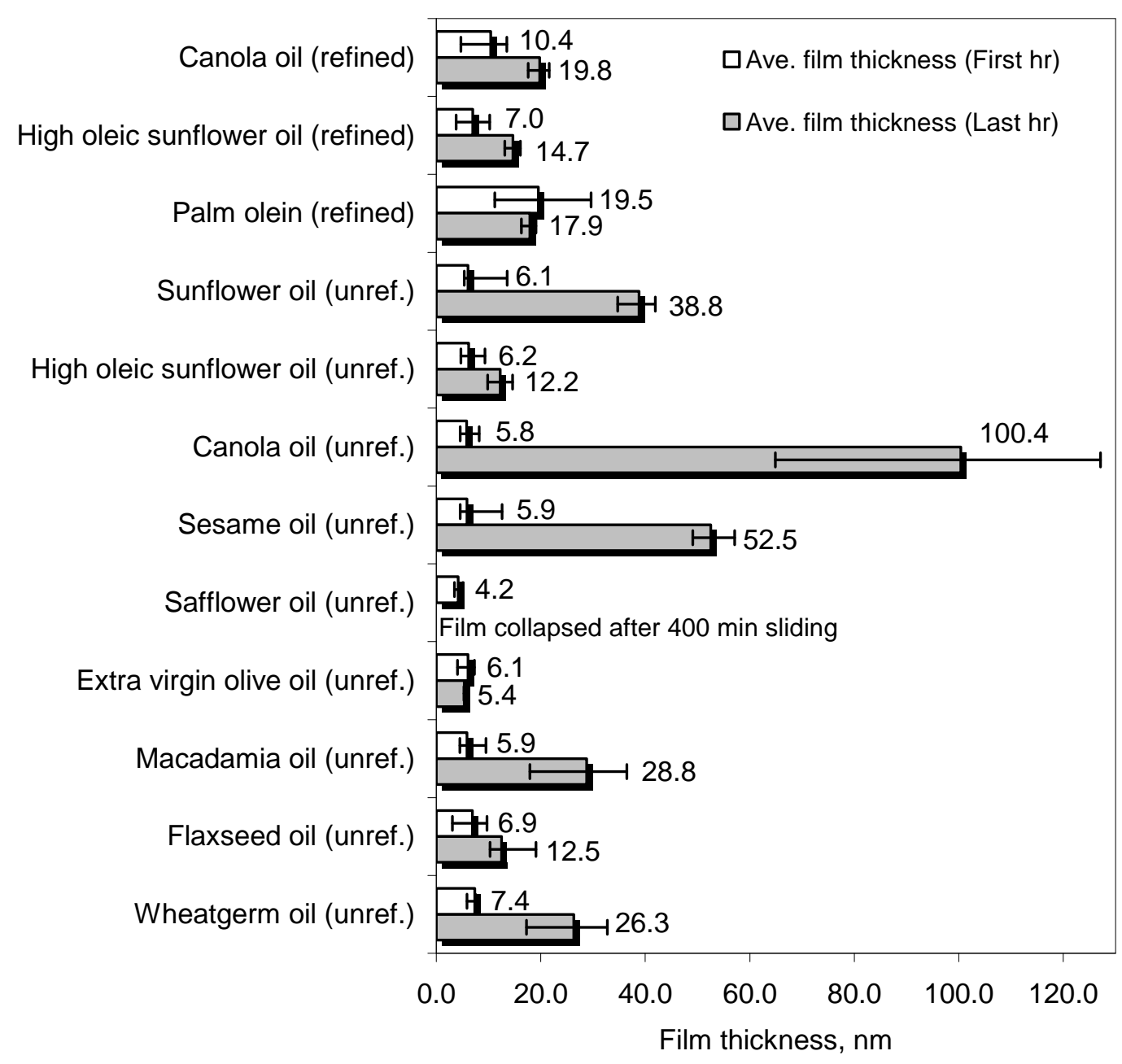

Fig. 5.3: Bar chart showing the mean film thicknesses for the first and last hour of the sliding tests. Error bars denote the min. and max. values of the data averaged at 15 min intervals from three repeat experiments.

\subsubsection{Ball Wear Scars and Wear Tracks}

Fig. 5.4 shows the optical microscope images taken of the ball wear scars and wear track. Except for high oleic sunflower oil (refined) and safflower oil, the diameter of the ball wear scars for all the other oils were very close to the calculated Hertzian contact diameter. On the other hand, the breakdown of the film for safflower oil caused accelerated wear to occur and this is evident in the wear scars which were more than three times larger than the others. Some scratches can also be observed on the ball wear scars of palm olein and wheatgerm oil, 
which can be attributed to the higher amount of insoluble matter that was present in the oils.

The reddish brown colour observed on the wear tracks, particularly of unrefined oils, appears to be of some corrosion products caused by chemical attack.
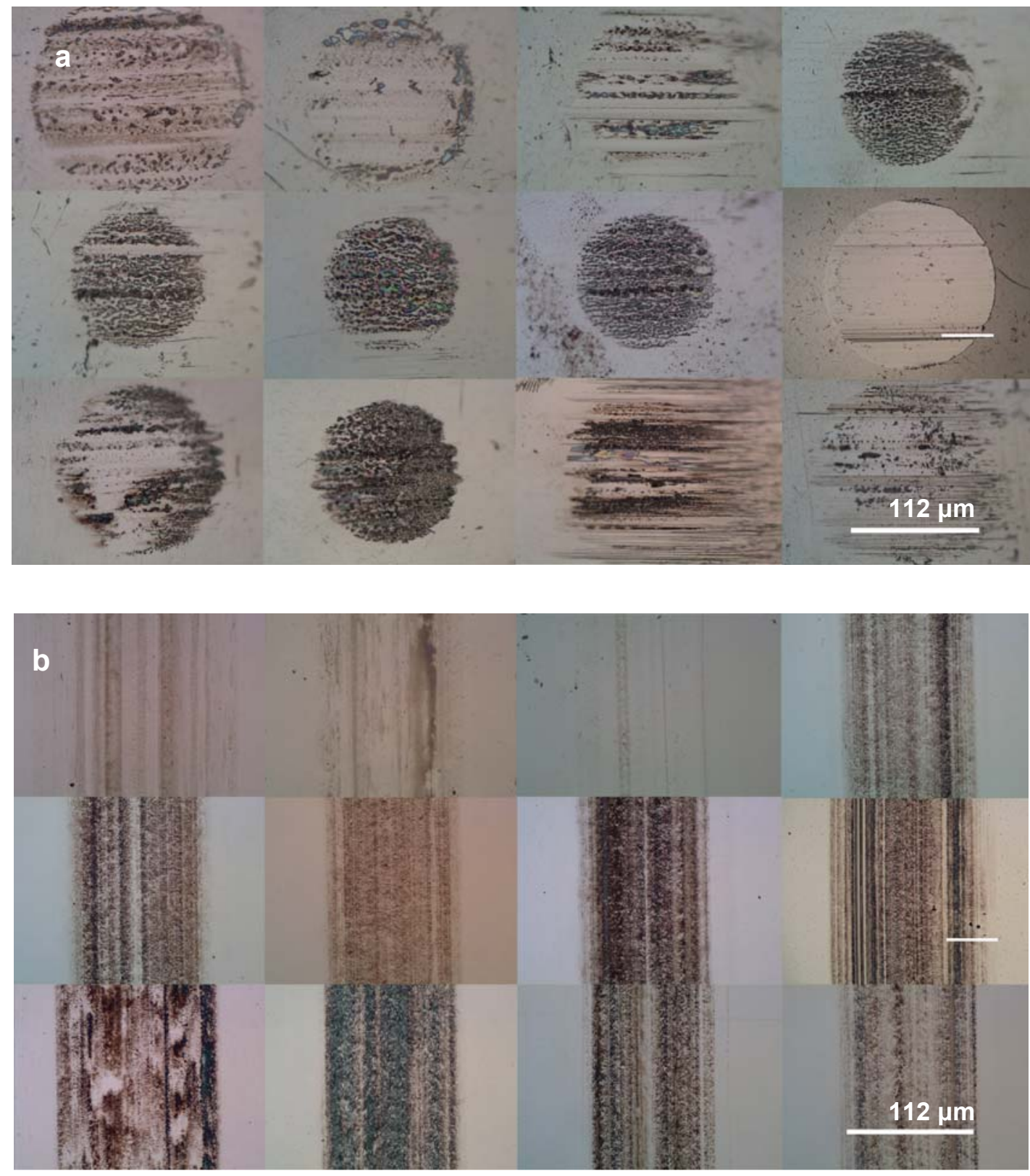

Fig. 5.4: Optical microscope images of a) ball wear scars and b) wear tracks. From left to right in descending rows: Refined oils - sunflower oil, canola oil and palm olein; Unrefined oils - sunflower oil, high oleic sunflower oil, canola oil, sesame oil, safflower oil, olive oil, macadamia oil, flaxseed oil and wheatgerm oil. The reference magnification bars indicating $112 \mu \mathrm{m}$ (corresponding to the calculated Hertzian contact diameter) applies to all the images, except for those of safflower oil (middle row, rightmost), where separate reference bars, denoting the same length, are shown. 
Surface profile measurements taken after the tests for these oils reveal that there was no significant change in the surface roughness, indicating that the visible scars are mainly due to chemical, and not mechanical wear. This is consistent the lack of any increase in the wear scar diameters from the calculated Hertzian contact diameters. For the case of safflower oil, where a film collapse occurred during the test, the change in the apparent area of contact had no effect on the film thickness calculation, since capacitances were not measurable after the film breakdown.

At higher magnifications of the ball wear scars of unrefined oils, patches could be seen distributed randomly on the wear scar (see Fig. 5.5). It should be noted that these patches remained adsorbed on the surface, even after washes with hexane and ethanol. The optical interference effect observed on these patches is a clear indication that these patches were organic in nature. Similar patches were, however, not visible on the wear tracks.

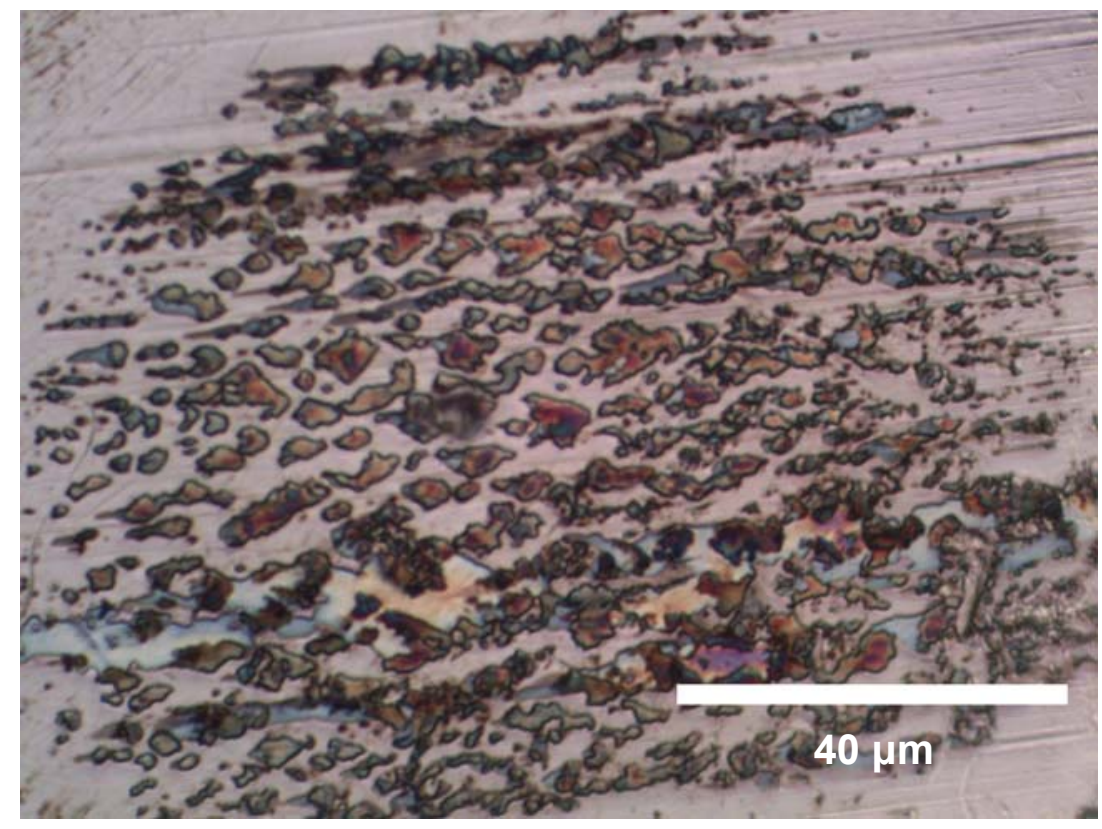

Fig. 5.5: Optical microscope image of the ball wear scar for unrefined sunflower oil. 


\subsubsection{Transition to Stick-Slip}

Of the oils tested, canola oil, sunflower oil and sesame oil (all unrefined oils) had the highest film growth rate. On closer examination, it appears that these oils experienced a relatively abrupt increase in the COF from roughly 0.1 to 0.12 during film growth and this increase in friction seemed to occur when their films grew thicker than $20 \mathrm{~nm}$ (see circled areas in Fig. $5.2 \mathrm{~b}$ and $5.2 \mathrm{c}$ ). Observation of the actual friction traces during each disc revolution indicates that after this occurred, friction changed from a 'smooth' type to a 'stick-slip' type (Fig. 5.6). As duly noted by Israelachvilli and workers [7-8], the occurrence and behaviour of the stickslip friction is dependent on the mass and stiffness of the friction force measuring system. Since this transition to stick-slip occurred without any change in the tribological conditions applied, a change in the physical state of the film must have occurred, possibly from being more liquid-like to either an amorphous or solid-like structure. The argument that this was due to metallic welding of the asperities is rejected, since a stable film of at least $20 \mathrm{~nm}$ had already developed between the surfaces, when this transition occurred.

The increase in friction with film thickness was, however, not observed for all the oils tested. The inverse was true for safflower oil and refined sunflower oil (tested earlier in ref. 12). For these oils, the friction decreased with increasing film thickness (Fig. 5.7). Incidentally, the films of these oils were the only ones that broke down catastrophically during the sliding test, causing severe wear to occur. It is also uncertain why this occurred and why the friction-film thickness relationship for these oils are different from the other oils. 


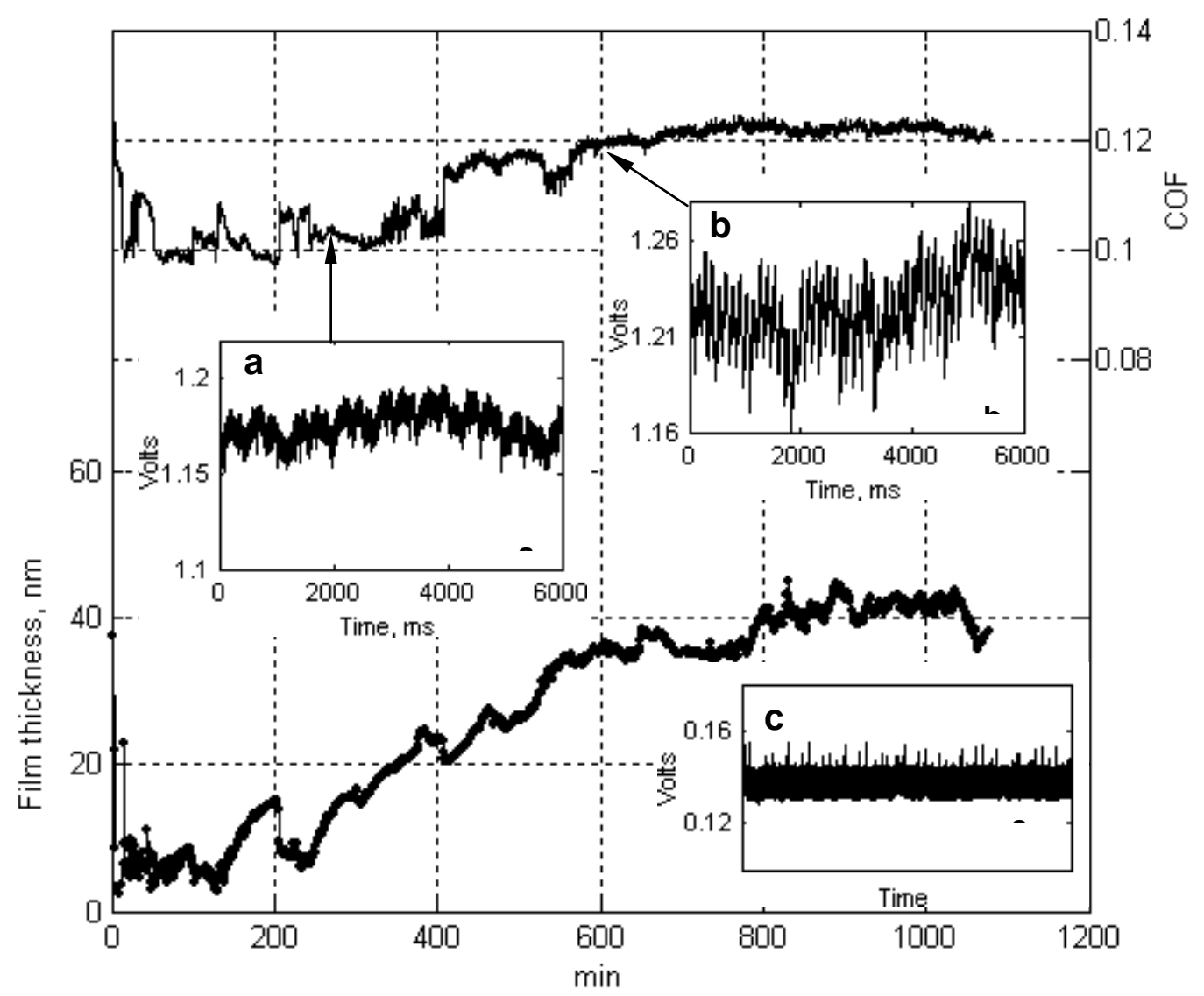

Fig. 5.6: Friction and calculated film thickness traces for unrefined sunflower oil. Data points are averaged over one complete revolution of the disc. Insets show actual reproductions of the voltage signals from the strain gauge amplifier plotted over one disc revolution at a sampling interval of 1000 samples/s. The friction was initially smooth (inset a). The transition to stick-slip friction occurred at approximately $20 \mathrm{~nm}$ film thickness. This transition did not occur instantly, but began over a portion of the wear track, and then gradually lengthens over several cycles until the entire wear track is of stickslip friction (inset b). The actual voltage signal under static conditions is shown in inset $\mathrm{c}$ for comparison. 


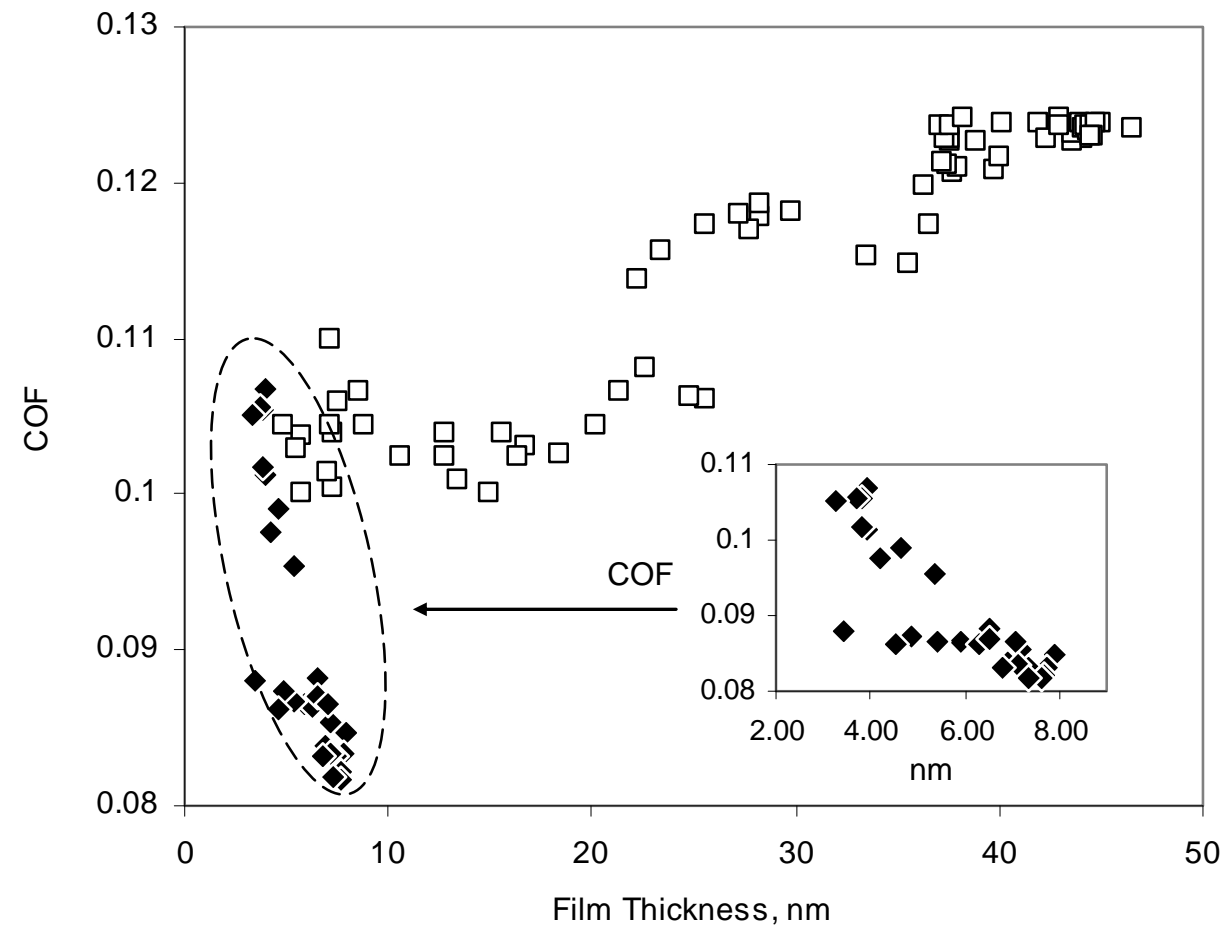

Fig. 5.7: Plot showing the difference in film thickness-friction relationship between unrefined safflower oil $\downarrow$ and unrefined sunflower oil $\square$. Inset shows the same plot for safflower oil over a smaller scale.

\subsubsection{Viscoelastic-like Behaviour at 'Stop'}

An interesting observation was made when the test was stopped. When the sliding speed was reduced to zero at the end of the 18-hr sliding test, the films of unrefined canola oil, sunflower oil and sesame oil were not squeezed out from the contact, but remained thick and continued to separate the surfaces for long periods of time. It was suggested that some of these films might possibly be frozen in their solid state as long as the load is not relieved. Once the load is relieved, the molecules would tend to become disordered and liquid-like again. This was, however, not observed in the films discussed here. When the contact was unloaded for several minutes and then loaded again, there was little or no change in the thickness of the film. The same was observed even when the contact was unloaded for more than $48 \mathrm{hrs}$. This indicates 
that the thick film did not dissolve into the bulk oil over time, but continued to be either solid-like or polymer-like, even under ambient pressures.

Furthermore, the friction did not immediately reduce to a constant value when sliding stopped, but decreased at a decreasing rate to a stable value over 2-3 hours. This timedependent behaviour at 'stop' bears some similarity with the viscoelastic behaviour generally encountered with entangled-chain polymers. To ensure that this observation was not due to the damping characteristics of the friction force measurement system itself, the 'stop' behaviour was compared with the case where metallic adhesion is expected to occur - a sliding contact between rough surfaces lubricated by paraffinic oil, as shown in Fig. 5.8. It is clear from this comparison that the damping effect was largely due to the sunflower oil boundary film. Further experiments to investigate these properties will be conducted in the future.

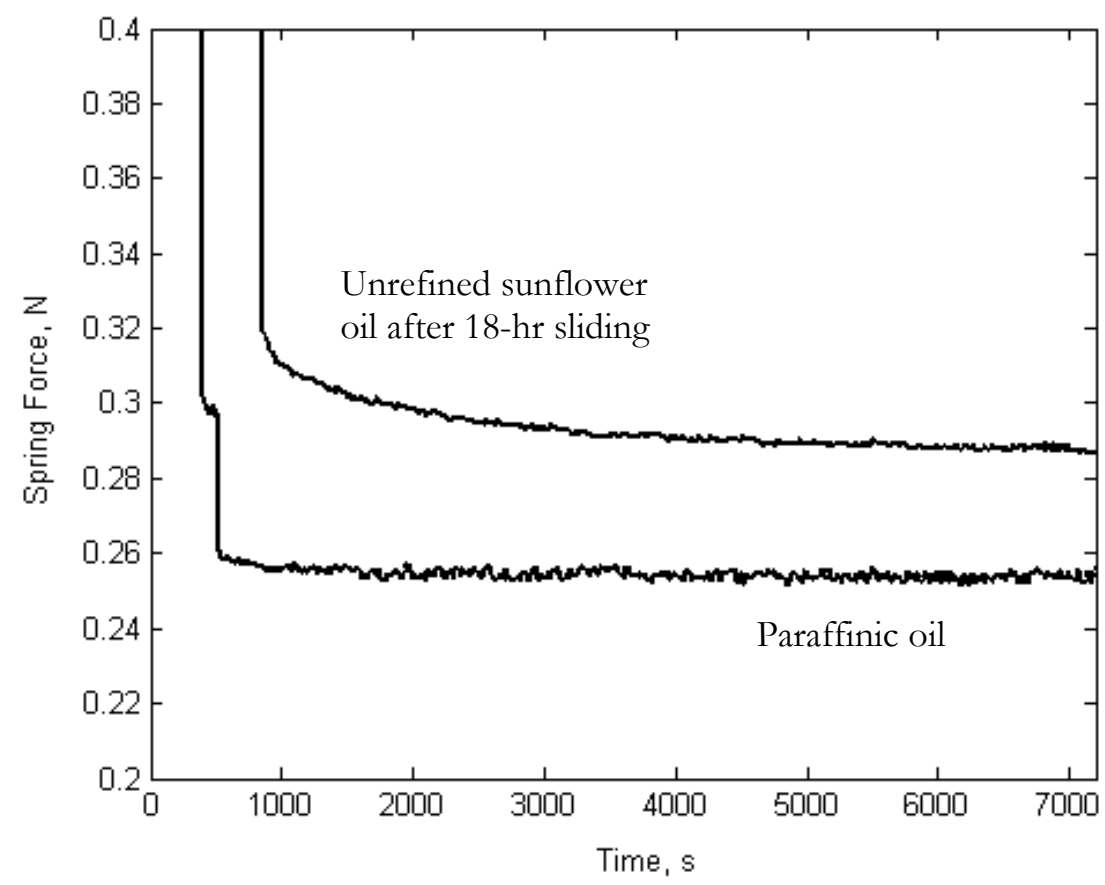

Fig. 5.8: Actual spring force traces when speeds are reduced to zero for paraffinic oil and unrefined sunflower oil. 


\subsubsection{Effect of Sudden Changes in Sliding Speeds}

Further experimentation revealed more time-dependent behaviours exhibited by the tribochemically formed boundary films. Thus far, sliding speed had not been a variable factor in the experiments conducted, as all tests have so far been conducted under a fixed sliding speed of $0.02 \mathrm{~m} / \mathrm{s}$. During one of the tests conducted for sunflower oil, the tribometer was intentionally left running after 18 hours of sliding. Hydrodynamic theory suggests that an increase in speed would increase the thickness of the film. Surprisingly, when the sliding speed was doubled, it was observed that there was an instant increase in the film thickness, followed by a gradual decrease over time. This transient behaviour could be due to the melting and solidification processes of the boundary films when sudden changes to the sliding speeds have been applied. Further tests were conducted to verify these behaviours.

As shown in Fig. 5.9, when the speed was increased to $0.04 \mathrm{~m} / \mathrm{s}$, there was no change in friction. At $0.12 \mathrm{~m} / \mathrm{s}$ and $0.24 \mathrm{~m} / \mathrm{s}$, however, there was a positive spike in the film thickness, which is accompanied by a sudden drop in friction. A gradual decrease in film thickness This spike was observed every time the speed was increased. The size of the spike was relative to the increase in sliding speed. This sudden increase in film thickness may possibly be due to volumetric expansion of the film and Couette flow as a result of the film melting, indicated by the smooth friction observed in inset a.

After the spike, a gradual decrease in film thickness follows, while the rate of the frictional decrease gradually reduces to zero. The smooth friction observed just after the change in sliding speed will remain for a few minutes before some of the stick-slip friction was observed again (inset b). It is believed that the film may be in the slow process of solidifying.

On reducing back to $0.02 \mathrm{~m} / \mathrm{s}$, there was a sudden drop in the film thickness, accompanied by an immediate increase in friction. At this stage, the film might have completely solidified, as evidenced by the return of the stick-slip behaviour (inset c). It is 
believed that when the speed was reduced, part of the original film was lost as a result of the melting during high speed sliding. However, as the test continued under the original slow sliding test speeds of $0.02 \mathrm{~m} / \mathrm{s}$, the film gradually recovered, though at a rate much faster compared to that of the initial film growth during the 18-hr sliding process.

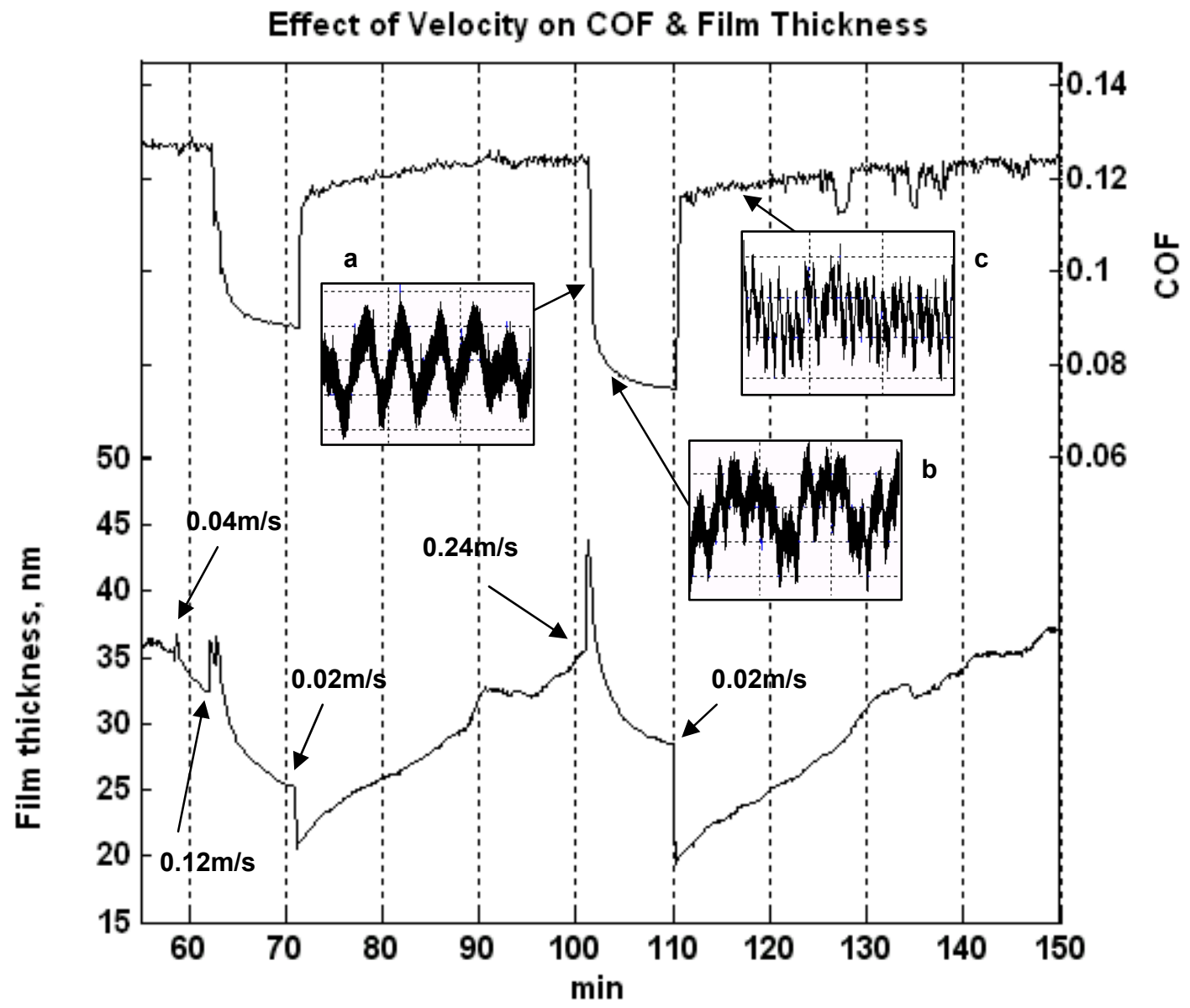

Fig. 5.9: Plot showing the change in friction and film thickness when the sliding speed is varied after the 18-hr sliding test at $0.02 \mathrm{~m} / \mathrm{s}$.

It is still not completely clear this stage why such transient behaviours are observed. It should however, be noted that the effect of altering the sliding speed may have an effect on the temperature at the area of contact and affects the calculation of film thickness. An increase in sliding speed should, theoretically increase contact temperatures, which would decrease 
dielectic constants. This has the effect of over reporting the actual film thickness. However, the results showed otherwise, whereby the film thickness gradually decreases when subjected to higher sliding speeds. Hence, the observed decrease in film thickness cannot be due to temperature effects.

\subsection{DISCUSSION}

Initially, there was a concern that the unexpected film growth could be due to the increase of viscosity as a result of oxidation of the bulk oil. A sample of canola oil (unrefined), flaxseed oil and high oleic sunflower oil (unrefined) were thus extracted from the lubricant cell after the 18-hr test and their viscosities were tested again. There seems to be no significant change in the viscosities to suggest that this was so. Another concern was that the apparent increase in film thickness was merely due to changes in the dielectric constant as a result of chemical changes of the film. This is unlikely, since tribochemical reactions leading to oxidation processes generally increase the polarity and dielectric constant, $\varepsilon$, of the film [9]. For a constant film thickness, an increase in $\varepsilon$ of the boundary film increases the measured capacitance and thus underestimates the actual film thickness. Thus, any apparent increase in the film thickness cannot be due to an increase in $\varepsilon$. Other concerns regarding the uncertainties of the dielectric constant are further discussed in ref. 12.

The lack of chemical attack on the ball wear scars and wear tracks of unrefined oils suggests that this chemical attack might be due to other components in the unrefined oils that are not present in the refined oils. Unrefined oils contain a high level of minor components (approx. 2-5\%) that are considerably reduced during the refining process. These include free fatty acids, trace metals, peroxides, water, phospholipids and pigments. Some of the natural anti-oxidants such as tocopherols, sterols, phenolic compounds and carotenoids are also destroyed or removed as a result [10]. As seen from Table 2, the amount of FFAs in unrefined oils is generally more than 10 times that found in refined oils. With the exception of 
wheatgerm oil, it appears that the amount of FFAs in both refined and unrefined oils is consistent with the amount of chemical attack observed on the ball wear scars and wear tracks.

FFAs are more polar than triglycerides and are able to replace physically adsorbed triglycerides on active surface sites of the metallic surface to form chemisorbed metallic soap films [11]. They are also more susceptible to autooxidation than esterified fatty acids and their presence have been known to accelerate the rate of oxidation in plant oils. The hydrophilic carboxyl ends of FFAs do not easily dissolve in the hydrophobic bulk oil and reside mainly at the liquid-air and liquid-solid interfaces [12]. The situation is further aggravated by the presence of water, which has the tendency to hydrolyze the triglycerides to produce more free fatty acids. The oxidation of lipids produces reactive oxygen species, hydroperoxides $\left(\mathrm{H}_{2} \mathrm{O}_{2}\right)$ and lipid peroxides (LOOH), which are known to readily react with ferrous oxide to form ferric oxides, with $\mathrm{LOOH}$ almost ten times more reactive than $\mathrm{H}_{2} \mathrm{O}_{2}$ [13]. The chemical attack visible on the wear scars thus supports the view that these oxidative processes occurred and were concentrated in the areas subjected to sliding contact.

A possible explanation for the increase in film thickness, for the case of unrefined canola oil, sesame oil and sunflower oil, is the fractionation of more polar and viscous compounds, produced as a result of accelerated oxidation processes in the sliding contact. Fractionation was earlier proposed by Biresaw [14-15]] to explain the deviation from EHL theory at higher than expected entrainment velocities. This would essentially imply that the thick films observed for some of the oils tested are, in fact, fully flooded EHL films. However, the absence of a loss in film thickness when speeds are reduced to zero, contradicts this theory, since any hydrodynamic effect would be lost and the film would thus be reduced to the thickness of the adsorbed layer. The theory of fractionation would therefore not be valid in our present case. 
A more likely reason for the film growth would be the polymerization of the triglycerides. If oxidative processes were accelerated in the areas of sliding contact, it is highly probable that chain branching processes by polymerization could also have occurred. High pressures have been known to induce a thermodynamically unstable system that promotes chemical bonding, and have been utilized in the polymerization of unsaturated organic compounds, such as acethylene and butadiene [16]. One of the polymerization pathways for fatty acids and lipids is by addition polymerization, which occurs by the attachment of the carboxyl end of a free fatty acid to the double bond of another fatty acid chain $[5,12]$. This produces high molecular weight polymeric compounds that would have much higher polarities than their parent triglycerides. The presence of iron catalysts, water, high pressures and temperatures are known to favour the polymerization process [12]. Incidentally, these conditions are inherently prevalent in a metal-to-metal sliding contact. A polymerization process that was concentrated at the areas of sliding contact would thus explain the thickening of the film. This would also explain the viscoelastic-like behaviour and the sustainability of the confined films when speeds are reduced to zero.

If the rate of polymerization increases with a greater number of double bonds (higher degree of unsaturation) in the plant oil, one would expect that monounsaturated oils would have poorer film growth ability than that of polyunsaturated oils. However, as previously noted, there seems to be no correlation between the film growth and the oil's unsaturation. Highly polyunsaturated oils, such as flaxseed oil, showed little film growth, while high oleic oils, such as macadamia oil, showed appreciable film growth compared to the other monounsaturated oils. Similarly, there was no significant evidence to suggest that oils with a higher amount of FFAs levels have a greater film growth rate. Unrefined canola oil, which exhibited the greatest film growth, contains the least amount of FFAs ( $0.1 \%$ oleic acid) among 
the unrefined oils tested. Other factors, such as the amount of water, transition metals and anti-oxidants, which could affect the oil's tendency to polymerize, must thus be considered.

\subsection{CONCLUSIONS}

The chemical attack observed on the wear scars, the viscoelastic-like behaviour of the film and the absence of a reduction in film thickness when speeds are reduced to zero, support the view that polymerization of the triglycerides was the cause of the film growth. However, the lack of correlation between the growth rate and the degree of unsaturation suggests that there could be possibly other mechanisms at work. There is, however, no doubt, that the films of some oils grow to thicknesses much greater than what is predicted from either EHL theory or their absorbed molecular heights. There is also some indication that some unrefined oils could form thicker films than refined oils, and would thus possess better anti-wear properties. This would be of particular interest to plant oil-based lubricant manufacturers, since a large portion of the cost is inevitably due to the refining process. The ability of some plant oil films to grow with sliding distance to thicknesses several times their molecular lengths is indeed remarkable. If the mechanisms of this film growth could be understood, plant oils with superior anti-wear properties can be developed, without excessively relying on anti-wear additives. Further studies will be conducted in the future to investigate the chemical structure and tribological properties of these films and to elucidate the mechanism of their growth. 


\section{ADDITIONAL MATERIAL: ELEMENTAL COMPOSITION USING ICP-MS}

After the experiments described in the preceding section, ICP-MS was conducted on 10 plant oils (refined oils - sunflower oil, high oleic sunflower oil, canola oil; unrefined oils - sunflower oil, high oleic sunflower oil, canola oil, safflower oil, olive oil, flaxseed oil and macadamia nut oil) at Amdel Laboratories Pty Ltd according to ASTM D5185. This was done in light of the fact that no correlation could be found between the fatty acid composition and the film growth behaviour of the oils.

The results of the ICP-MS test are shown in Table 1. Elements with less than $1 \mathrm{ppm}$ detected in all the oils are not shown. The film growth ratios and iodine values gathered from the previous work [1] are also shown in the table for comparison. It can be seen from the table that the oils with the highest film growth - unrefined canola oil and macadamia oil contained a significant amount of phosphorus and calcium compared to the other oils. Macadamia nut oil, which has the highest phosphorus levels (36 ppm), exhibited a film growth approximately twice that of the unrefined high oleic sunflower oil and olive oil. Similarly, among the highly polyunsaturated plant oils $(\mathrm{IV}>120)$, only the boundary films of flaxseed oil and sunflower oil (both unrefined and contained 9 and 6 ppm of phosphorus) were stable, and did not collapse during the sliding test. It would thus appear that there is some relationship between the film growth and the phosphorus and calcium content in the oils.

The phosphorus content for both unrefined and refined canola oil is similar to that found in a study by Prior et al. [17]. It is likely that the phosphorus found in the oils originated from phospholipids. Studies have shown that the quantity of phospholipids in plant oils can be estimated by applying a conversion factor of $\sim 24$ to the phosphorus content in ppm [18]. The unrefined canola oil therefore contains approximately $0.05 \mathrm{wt} \%$ phospholipids. The exact 
role of calcium in the tribological process is uncertain but the element has been known to act as a binder to phospholipids by neutralizing the phosphate group, which renders the phospholipid cationic and highly polar [19-20].

Table 5.4: ICP-MS results with the iodine value (IV) and film growth ratio of 10 plant oils

\begin{tabular}{|c|c|c|c|c|c|c|c|c|c|c|}
\hline \multirow{2}{*}{ Oils } & \multicolumn{8}{|c|}{ Elements (ppm) } & \multirow{2}{*}{$\begin{array}{l}\text { Iodine } \\
\text { Value } \\
\text { (IV) }\end{array}$} & \multirow{2}{*}{$\begin{array}{c}\text { Ave. } \\
\text { film } \\
\text { growth } \\
\text { ratio }^{2}\end{array}$} \\
\hline & $\mathrm{Fe}$ & $\mathbf{N a}$ & $\mathbf{P}$ & Si & Bo & $\mathrm{Ca}$ & $\mathbf{M g}$ & $\mathrm{Zn}$ & & \\
\hline \multicolumn{11}{|l|}{ Refined Oils } \\
\hline HO Sunflower Oil & $<1$ & $<1$ & $<1$ & $<1$ & $<1$ & $<1$ & $<1$ & $<1$ & 84 & 2.1 \\
\hline Canola Oil & 1 & $<1$ & 4 & $<1$ & $<1$ & 2 & 1 & 1 & 115 & 2.2 \\
\hline Sunflower Oil & $<1$ & $<1$ & $<1$ & $<1$ & $<1$ & $<1$ & $<1$ & $<1$ & $\sim 131$ & $\mathrm{NA}^{1}$ \\
\hline \multicolumn{11}{|l|}{ Unrefined Oils } \\
\hline Macadamia Nut Oil & 1 & $<1$ & 36 & $<1$ & 2 & 12 & 10 & $<1$ & 73 & 3.9 \\
\hline HO Sunflower Oil & $<1$ & $<1$ & 1 & 1 & $<1$ & 1 & $<1$ & $<1$ & 93 & 1.7 \\
\hline Canola Oil & $<1$ & 1 & 20 & $<1$ & $<1$ & 31 & 5 & $<1$ & 110 & 15 \\
\hline Sunflower Oil & $<1$ & $<1$ & 6 & $<1$ & $<1$ & 3 & 2 & $<1$ & 131 & 4 \\
\hline Safflower Oil & $<1$ & $<1$ & 1 & $<1$ & $<1$ & 1 & $<1$ & $<1$ & 135 & $\mathrm{NA}^{1}$ \\
\hline Olive Oil & $<1$ & $<1$ & $<1$ & $<1$ & $<1$ & $<1$ & $<1$ & $<1$ & 77 & 1 \\
\hline Flaxseed Oil & $<1$ & 30 & 9 & $<1$ & $<1$ & 5 & 3 & $<1$ & 176 & 2.3 \\
\hline
\end{tabular}

1. Film collapsed during the sliding test.

2. Ratio of the final film thickness to the initial film thickness 


\section{REFERENCES}

1. Biresaw, G.: Elastohydrodynamic properties of seed oils. J. Am. Oil Chem. Soc. 83(6), 559$566(2006)$

2. AOAC: Official Methods of Analysis, $15^{\text {th }}$ ed. Association of Official Analytical Chemists, Inc., Washington, DC (1990)

3. Kaatze, U.: Reference liquids for the calibration of dielectric sensors and measurement instruments. Meas. Sci. Technol. 18, 967-976 (2007).

4. Maryott, A.A., Smith, E.R.: Table of Dielectric Constants of Pure Liquids (NBS Circular 514), Natl. Bur. Stand, New York (1951)

5. Thomas, A.: Fats and Fatty Oils. In Gerhartz, W., Yamamoto, Y.S., Kaudy, L., Rounsaville, J.F., Schulz, G. (eds.), Ullman's Encyclopedia of Industrial Chemistry, $5^{\text {th }}$ edn., VCH Verlagsgesellschaft mbH, Weinheim, Vol. A10, pp 173-243 (1987)

6. Knothe, G.: Structure indices in FA chemistry. How relevant is the iodine value? J. Am. Oil Chem. Soc. 79(9), 847-854 (2002)

7. Homola, A.M., Israelachvili, J.N., Gee, M.L., McGuiggan, P.M.: Measurements of and relation between the adhesion and friction of two surfaces by molecularly thin liquid films. J. Tribol. 111(4), 675-682 (1989)

8. Heuberger, M., Luengo, G., Israelachvili, J.N.: Tribology of shearing polymer surfaces. 1. Mica sliding on polymer (PnBMA). J. Phys. Chem. B 103, 10127-10135 (1999)

9. El-Shami, S.M., Zaki Selim, I., El-Anwar, I.M., Hassan El-Mallah, M.: Dielectric properties for monitoring the quality of heated oils. J. Am. Oil Chem. Soc. 69(9), 872-875 (1992)

10. Gunstone, F.D.: Vegetable Oils. In Shahidi, F. (ed.), Bailey's Industrial Oil and Fat Products, $6^{\text {th }}$ edn., John Wiley \& Sons Inc., New Jersey, Vol. 1, pp 213-267 (2005) 
11. Beentjes, P.C.J., Van Den Brand, J., De Wit, J.H.W.: Interaction of ester and acid groups containing organic compounds with iron oxide surfaces. J. Adhesion Sci. Technol. 20(1), 1-18 (2006).

12. Choe, E., Min, D. B.: Mechanisms and factors for edible oil oxidation. Food Science and Food Safety. 5(4), 169-186 (2006)

13. Halliwell, B., Gutteridge, J.M.C.: Free Radicals in Biology and Medicine. $3^{\text {rd }}$ edn. Oxford University Press, New York (1999)

14. Biresaw, G.: Elastohydrodynamic properties of seed oils. J. Am. Oil Chem. Soc. 83(6), 559$566(2006)$

15. Biresaw, G., Bantchev, G.: Effect of chemical structure on film-forming properties of seed oils. J. Syn. Lubri. 25, 159-183 (2005)

16. Schettino, V., Bini, R.: Molecules under extreme conditions: Chemical reactions at high pressure. Phys. Chem. Chem. Phys. 5, 1951-1965 (2003)

17. Prior, E. M., Vadke, V. S., Sosulki, F. W. (1991). Effect of heat treatments on canola pressed oils. I. Non-triglyceride components. J. Am. Oil Chem. Soc. 68(6), 401-406

18. Carelli, A. A., Ceci, L. N., Crapiste, G. H. (2002). Phosphorus-to-Phospholipid conversion factors for crude and degummed sunflower oils. J. Am. Oil Chem. Soc. 79(12), 1177-1180

19. Hills, B. A. (2002). Surface-active phospholipid: a Pandora's box of clinical applications. Part I. The lung and air spaces. Intern. Med. J., 32, 170-178.

20. Shah. D. O., Schulman, J. H. (1967). The ionic structure of lecithin monolayers. J. Lipid Research. 8, 227-233. 


\section{The Chemical Nature of Canola Oil Boundary Films}

\subsection{INTRODUCTION}

Despite the extensive experimental work conducted on the 13 plant oils, no correlation could be found between the fatty acid composition and film growth behaviour. Later tests using ICP-MS, however, suggest the possibility that phospholipids might play a role, but there is still insufficient evidence to support this hypothesis. It is envisaged that surface spectroscopic techniques such as Fourier transform infrared (FTIR) and Raman spectroscopy could provide more information regarding the chemical nature of these films and the mechanisms of its growth.

Both FTIR or Raman spectroscopy techniques complement each other and can be adapted for surface-sensitive chemical studies, namely the grazing angle RAIRS (reflectance adsorption infra-red spectroscopy) and SERS (surface-enhanced Raman spectroscopy) respectively. SERS is a powerful technique that is capable of providing molecular bonding information even from a single monolayer. The technique capitalizes on the phenomenon that the Raman signal intensity is enhanced $10^{2}$ to $10^{6}$ times when the substrate is microscopically roughened and made of a coinage metal, such as silver, gold or copper [1]. The unique feature of SERS is that the local-field enhancement decays rapidly from the surface, so that the technique is inherently biased towards the first layer of the adsorbate. In recent times, the 
detection limit was found to go as low as a single molecule [2-5]. For analyzing small sample areas (less than $100 \mu \mathrm{m}^{2}$ ), the SERS method is preferred over RAIRS since conventional Raman spectrometers can achieve spot sizes down to 2 um in diameter. The disadvantage of the SERS technique, however, is that the surface needs to be made of silver, copper or gold.

However, there are several challenges. The contact area to be analyzed is only approximately $100 \mu \mathrm{m}$ wide, and the minimum focal spot size of the instruments would ideally need to be at smaller than $10 \mu \mathrm{m}$. The other challenge is that the tribochemically-formed films generated are typically less than $100 \mathrm{~nm}$ thick, and are thus sensitive to the effects of irradiation under the laser beams of both FTIR and Raman techniques, causing permanent damage to the sample. Shorter exposure times however, lead to poor intensity, high noise signals which make interpretation of the analysis results difficult.

Our trials with normal FTIR technique alone showed that the films were too thin to be analyzed and signals obtained were very poor. The grazing-angle RAIRS technique was not tested, as the accessory needed for this technique to work was not available at the time. However, due to its high sensitivity to adsorbed molecules, it is envisaged the technique might be able to provide some useful spectroscopic information. Similarly, our earlier tests with using normal Raman spectroscopy technique on the uncoated bearing ball also did not reveal any discernible information. At longer exposure times, burning of the sample occurred and this was identified clearly by the D and $G$ peaks of amorphous carbon. Our experiments using the SERS technique by employing silver-coated bearing balls, however, produced much clearer results. The irradiation time could be increased to an extent that did not burn the sample.

Unfortunately, it was not possible to reproduce the same thick films $(20-100 \mathrm{~nm})$ as observed in the original experiments using uncoated steel balls on refined and unrefined canola oils. This is because the silver coating on the bearing ball was too soft and worn out quickly during the sliding tests. However, closer analysis of the spectra collected from the wear 
scars of the silver-coated bearing balls, revealed some very interesting and reproducible differences between the refined and unrefined canola oil spectra. Although the spectra obtained were sufficiently clear, analysis of the results proved to be a challenge. Assignment was relatively straightforward for some peaks since they are widely known and have been published widely. The difficulty lies in the fact that the tribological process may cause the generation of other species such as peroxides and aromatic compounds of which their SERS spectra is less known. A clearer understanding of the chemical nature of these boundary films on transition metals must therefore await the investigation using RAIRS and its comparison with SERS results. In this work, the SERS results are presented.

\subsection{EXPERIMENTAL SECTION}

\subsubsection{Test Lubricants}

Instead of testing a diverse set of plant oils in this work, it was decided that only the refined and unrefined canola oil would be tested. The two oils have been chosen as their observed film growth behaviours differ significantly, despite their similarity in fatty acid compositions. This allows an objective comparison to be made, as any differences in the SERS spectra obtained would most likely be due to that of the higher proportion of minor components present in the unrefined oil. The refined and unrefined canola oils were the same oils used in the experiments described in Chapter 5. They were obtained from Cargill Inc and Melrose Health Pty Ltd respectively.

\subsubsection{Tribological Test and Sample Preparation}

Silver electroplating of the bearing ball was done in a local silver plating shop. The thickness of the silver coating was measured to be approximately $0.02 \mathrm{~mm}$ using a digital vernier caliper. The apparatus used for the sliding experiments was an in-house developed ball-on-disc 
tribometer. The tribological conditions used were kept the same as that described in Chapter 5, except that the bearing ball used was replaced with a silver coated bearing ball (see Fig. 6.1). Briefly, the conditions were as follows:- sliding speed - $0.02 \mathrm{~m} / \mathrm{s}$; temperature $-40{ }^{\circ} \mathrm{C}$; load $-7 \mathrm{~N}$; duration/ total number of cycles - 18 hrs/ 10800 cycles; Material (disc) - 52100 steel hardened to 810 $\pm 10 \mathrm{HV}$ and polished to $13 \mathrm{~nm} \mathrm{Ra}$; Material (ball) -52100 bearing ball (8 $\mathrm{mm}$ dia.) with silver coating.

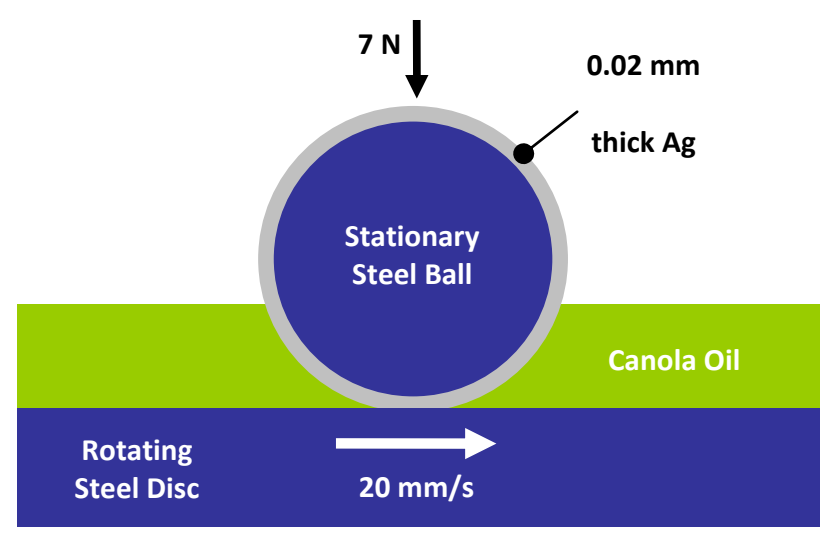

Fig. 6.1: Illustration of the ball-on-disc pure sliding contact under fully-flooded lubricated condition.

After each sliding test, the silver-coated bearing ball (still wet with a layer of the bulk oil) was carefully removed from the test apparatus and transferred to a sample holder. It was then stored by immersing it in the same oil used in the test and kept at approximately $4{ }^{\circ} \mathrm{C}$ in the refrigerator. This was done to prevent any oxidation that would have otherwise occurred if the layer of oil was exposed to the atmosphere for long periods of time. SERS was conducted not more than 12 hours from the time of storage. Prior to SERS, the sample holder with the bearing ball was removed from the bulk oil, washed briefly with hexane, and then allowed to dry under ambient conditions. The sliding experiment and SERS were also repeated to ensure reproducibility of the peaks obtained. 


\subsubsection{Surface Enhanced Raman Spectroscopy (SERS)}

The Raman spectrometer used was a Dilor Labram model 1B, equipped with a $30 \mathrm{~mW}$ He-Ne laser, coupled to an Olympus BX40 microscope. Raman spectra were recorded at different spots on the wear scar with $\sim 2.4 \mathrm{~cm}-1$ resolution over a spectral range of 3063 to $127 \mathrm{~cm}-1$, using the $632.8 \mathrm{~nm}$ laser line via a 50X microscope objective to focus onto a spot approximately $2 \mu \mathrm{m}$ in diameter. The $520.7 \mathrm{~cm}^{-1}$ line of silicon was used for wavelength calibration. A neutral density filter (D1 filter) was used to attenuate the laser power to $10 \%$ (0.4 $\mathrm{mW}$ at the sample). Under such conditions, it was found that any spot on the wear scar should not be irradiated for more than $150 \mathrm{~s}$, otherwise decomposition of the film would occur. An accumulation time of $60 \mathrm{~s}$ was chosen, thus allowing for a repeat spectrum to be collected at the same spot for comparison.

\subsection{RESULTS AND DISCUSSION}

It was observed that the silver coating on the bearing ball was too soft, and was continually being sheared away during sliding. Black deposits were also observed deposited around the wear scar after the sliding tests (Fig. 6.2). These black deposits were later found to be amorphous carbon in the form of aggregated soot. It is clear that the film growths observed in a lubricated steel-on-steel condition were not reproduced here with the silver-coated bearing ball as the counter surface. However, the SERS spectra obtained from the two oils showed persistent and reproducible differences in their features and these differences provided the basis for comparison in this study.

Initially, SERS was conducted on the wet sample, i.e., where the surface of the ball was still covered by a thin layer of the bulk oil. The spectra obtained for the unrefined oil were dominated mainly by fluorescence (not shown), presumably due to the presence of carotenoids. The fluorescence was less in the refined oils spectra, but the contribution of 
Raman scattering from the bulk liquid layer exceeded that that of the adsorbate in most cases. This is evident from the major bands which are characteristic of the normal Raman spectra of plant oils (Fig. 6.3) i.e. the $\mathrm{CH}_{2}$ and $\mathrm{CH}_{3}$ stretching modes $\left(2800-3000 \mathrm{~cm}^{-1}\right)$, symmetric rock in cis double bond $\left(1265 \mathrm{~cm}^{-1}\right)$, in-phase $\mathrm{CH}_{2}$ twist $\left(1305 \mathrm{~cm}^{-1}\right), \mathrm{CH}_{2}$ scissoring mode $(1442 \mathrm{~cm}$ $\left.{ }^{1}\right)$, cis double bond stretching $\left(1655 \mathrm{~cm}^{-1}\right)$ and ester stretching $\left(1747 \mathrm{~cm}^{-1}\right)$ [6-7]. In addition, weaker peaks near 235, 930, 1377 and $1560 \mathrm{~cm}^{-1}$ (labeled in figure) that are not usually typical of the normal Raman spectrum of plant oils were observed in some of the spectra collected.

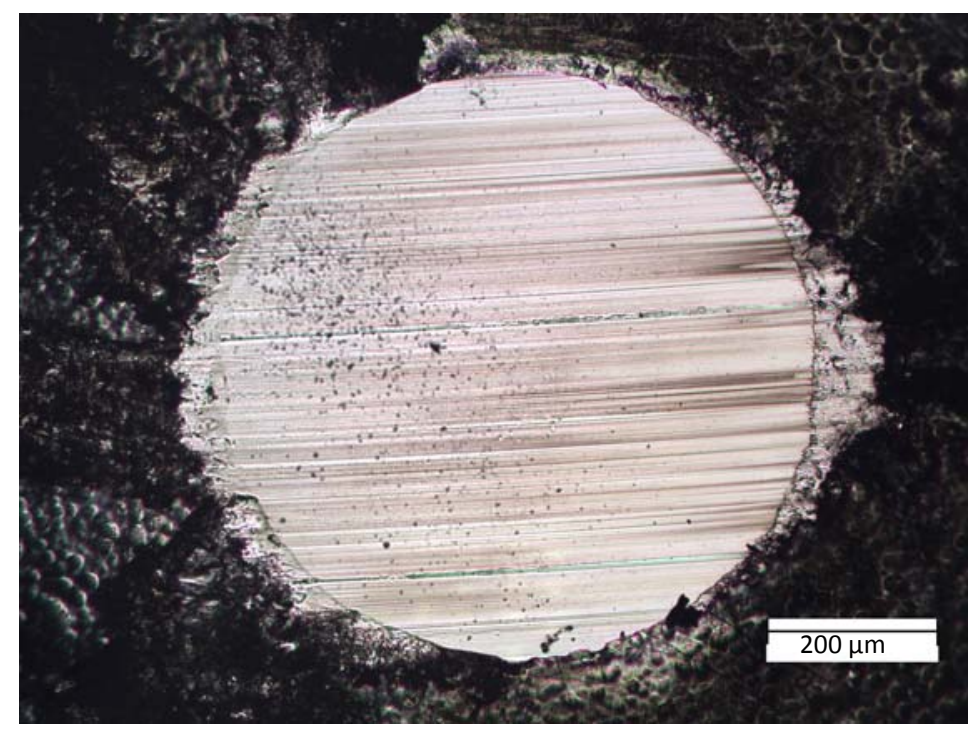

Fig. 6.2: Optical microscope image of the unwashed wear scar of the silver-coated ball for unrefined canola oil.

The first three peaks were later found to be due to the vibrations of the carboxylate groups, which were most likely enhanced due to its proximity with the silver surface. The superposition of Raman contribution from both the bulk liquid and adsorbate made it difficult to conclude whether the vibrations originate from the bulk liquid, the adsorbate or both. These problems were avoided once the bulk oil layer was removed with a non-polar solvent. 


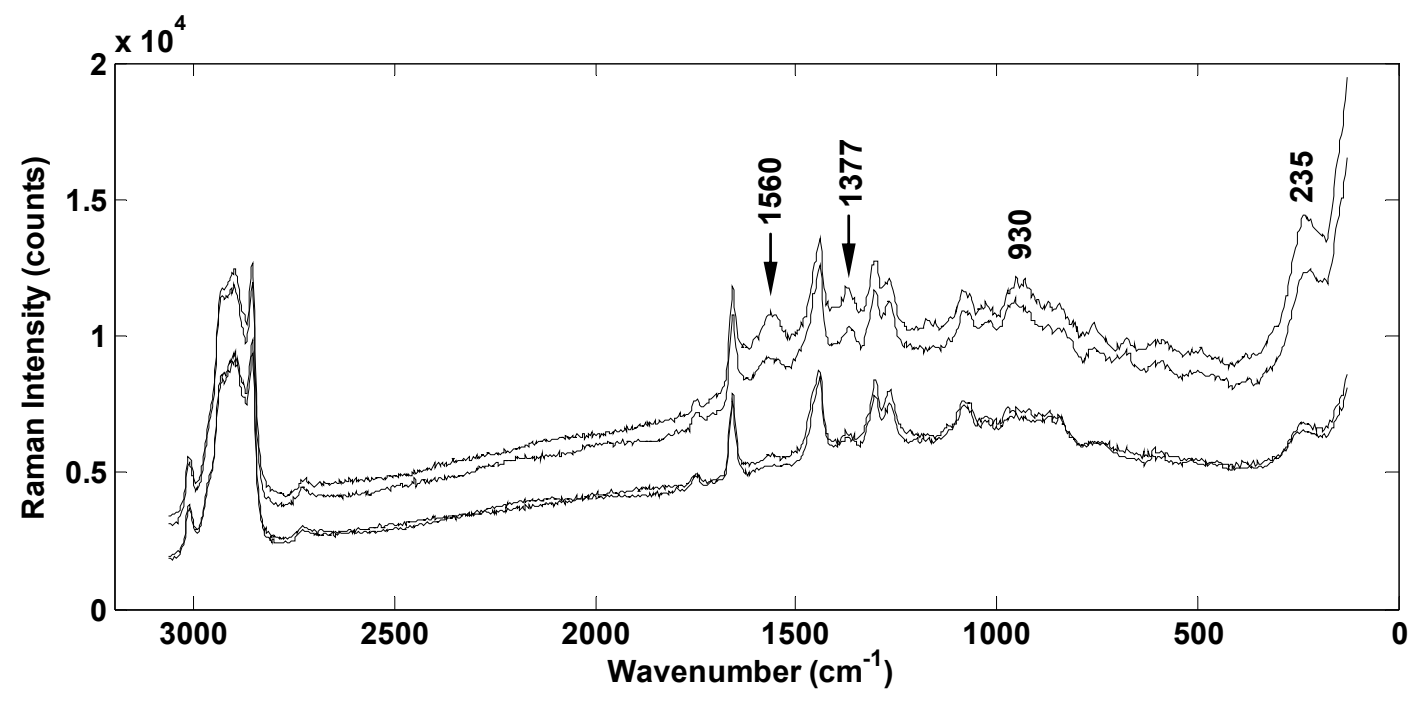

Fig. 6.3: SERS spectra of the unwashed ball wear scar of refined canola oil with enhanced vibrational bands (labelled).

It was observed that once the bulk oil layer was removed, many of the peaks associated with the normal Raman spectra of the bulk oil disappeared, leaving behind the contributions from the SERS effect. Assignment of the peaks identified from the spectra of both refined and unrefined canola oils are summarized in Table 1. It should be noted that the films were, however, more susceptible to the effects of photodecomposition due to excessive laser irradiation. Above $10 \mathrm{~s}$ of irradiation under the full laser power of $4 \mathrm{~mW}$, two new peaks can be observed near $1350 \mathrm{~cm}^{-1}$ and $1600 \mathrm{~cm}^{-1}$ and their intensities increased with irradiation time. These peaks are, without doubt, identified as the D and G peaks of amorphous carbon (Fig. 6.4) [8-12] and has been similarly observed in many previous SERS studies due to either photodecomposition or carbon contamination during surface preparation [4-5, 10]. Care was thus taken to ensure that the laser power and irradiation time was selected such that the highest intensities can be obtained without chemically altering or degrading the film. 


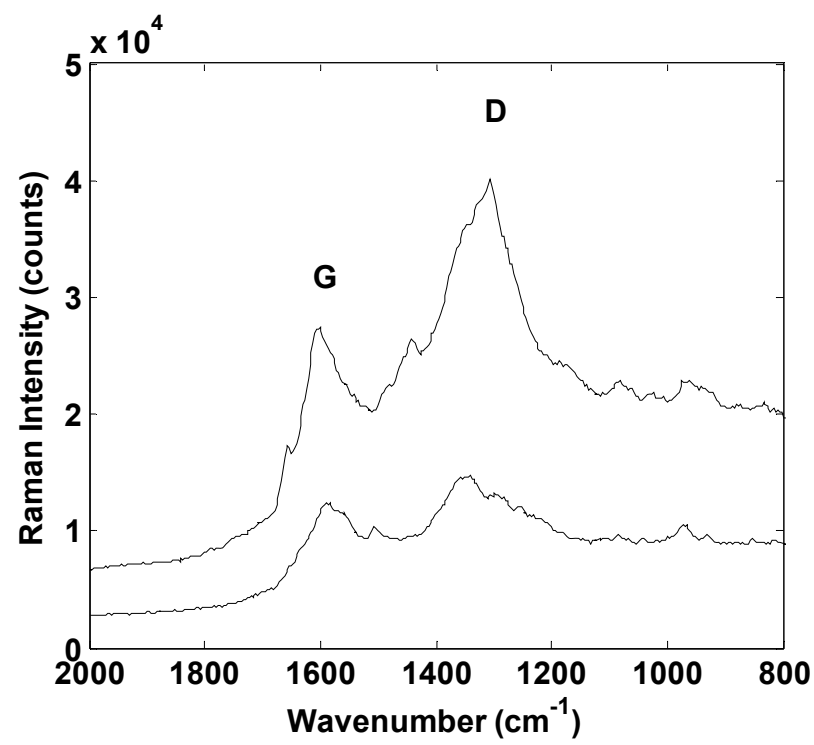

Fig. 6.4: SERS spectra for refined canola oil (full laser power $=4 \mathrm{~mW}$; irradiation time $=6 \mathrm{~s}$ ).

As a comparison, SERS spectra were also collected from a new silver-coated bearing ball, which had been immersed in the refined canola oil for $18 \mathrm{hrs}$ under ambient conditions. The Raman intensities of the spectra obtained were very poor, indicating that the canola oils did not form chemisorbed films with the silver surface. This is expected, since triglycerides are not known to form chemisorbed films on metallic surfaces, but are physically adsorbed onto the surface via their hydrogen bonds. This also shows that the spectra obtained from worn samples are due solely to a tribochemically-formed boundary film. 
Table 6.1: Assignment of the SERS spectra peak frequencies for refined and unrefined canola oil.

\begin{tabular}{|c|c|c|}
\hline \multirow{2}{*}{$\begin{array}{c}\text { Vibrational Band } \\
\text { Assignment }\end{array}$} & \multicolumn{2}{|c|}{ Peak Position, $\mathrm{cm}^{-1}$} \\
\hline & $\begin{array}{c}\text { Refined Canola } \\
\text { Oil }\end{array}$ & $\begin{array}{c}\text { Unrefined Canola } \\
\text { Oil }\end{array}$ \\
\hline$\nu(\mathrm{Ag}-\mathrm{O})$ & 235 & 215 \\
\hline $\mathrm{CH}_{3} \operatorname{rock}, \tau(\mathrm{C}-\mathrm{H})$ & 760 & 760 \\
\hline$\nu(\mathrm{C}-\mathrm{C})$ & & 892 \\
\hline \multirow[t]{2}{*}{$v\left(\mathrm{C}^{-} \mathrm{COO}^{-}\right)$} & 930 & 930 \\
\hline & 955 & \\
\hline$\delta(\mathrm{C}-\mathrm{H}), \nu(\mathrm{C}-\mathrm{O})$ & 1030 & \\
\hline$\nu(\mathrm{C}-\mathrm{C})_{\mathrm{G}}, \nu\left(\mathrm{PO}_{4}^{3-}\right)$ & & 1090 \\
\hline$\delta(\mathrm{C}-\mathrm{H}), \nu(\mathrm{C}-\mathrm{C})$ & 1167 & \\
\hline$\tau\left(\mathrm{CH}_{2}\right)$ & 1300 & 1295 \\
\hline$\nu\left(\mathrm{COO}^{-}\right)$ & 1377 & 1395 \\
\hline$\delta\left(\mathrm{CH}_{2}\right)$ & & 1439 \\
\hline$\nu(\mathrm{C}=\mathrm{C})$ aromatic ring & 1560 & \\
\hline$v(C=C)$ olefinic chain & 1630 & 1630 \\
\hline$v(\mathrm{C}=\mathrm{O})$ & 1700 & \\
\hline$v\left(\mathrm{CH}_{2}\right)_{\mathrm{S}}$ & & 2855 \\
\hline$v\left(\mathrm{CH}_{2}\right)_{\mathrm{A}}$ & & 2875 \\
\hline$v\left(\mathrm{CH}_{3}\right)$ & 2930 & 2930 \\
\hline
\end{tabular}



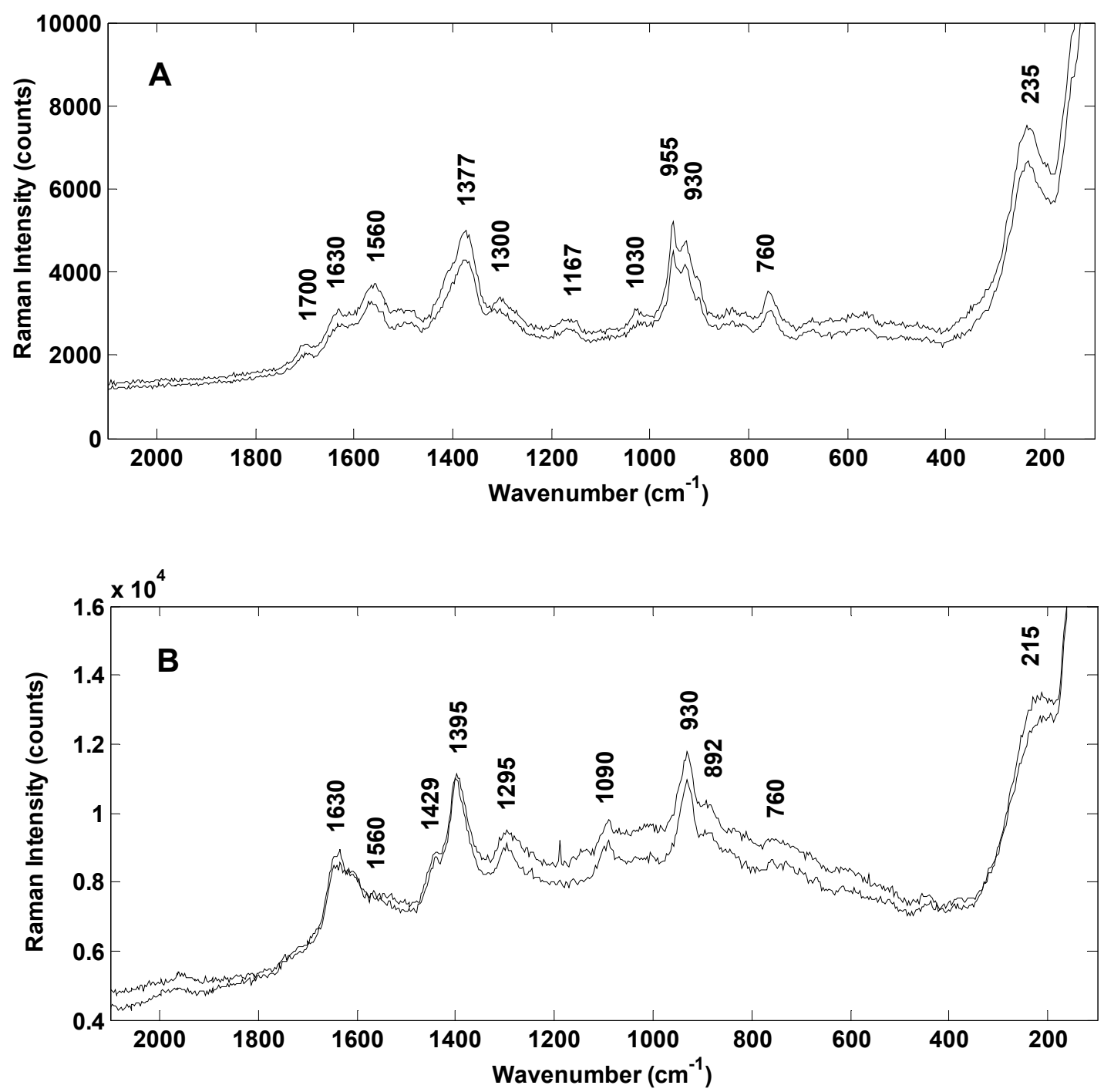

Fig. 6.5: SERS spectra (2100-100 $\mathrm{cm}^{-1}$ ) of ball wear scar (after hexane washing) for A. refined canola oil, B. unrefined canola oil. 


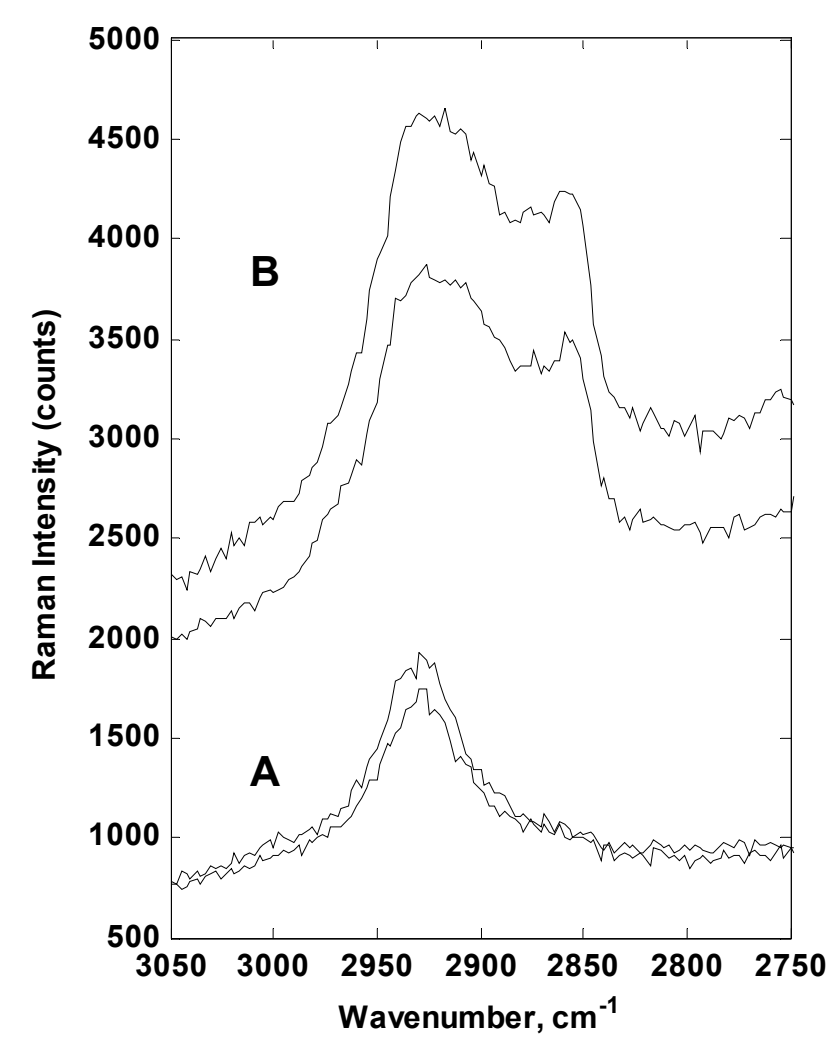

Fig. 6.6: SERS spectra (3050-2750 $\mathrm{cm}^{-1}$ ) of ball wear scar (after hexane washing) for A. refined canola oil, and B. unrefined canola oil

\subsubsection{Refined Canola Oil}

The strongest peaks appeared near 235, 940, 1377 and $2930 \mathrm{~cm}^{-1}$ for the case of refined canola oil (Fig. 6.5A). On closer examination, it appears that the $940 \mathrm{~cm}^{-1}$ peak was in fact a doublet at 930 and $955 \mathrm{~cm}^{-1}$. The doublet $\left(930,955 \mathrm{~cm}^{-1}\right)$ and the broad peak at $1377 \mathrm{~cm}^{-1}$ can be

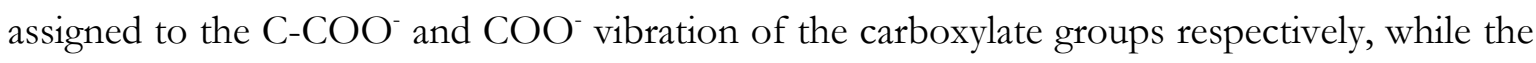
peak at $235 \mathrm{~cm}^{-1}$ can be assigned to the vibration of the $\mathrm{Ag}-\mathrm{O}$ bond [13-20]. It is uncertain at

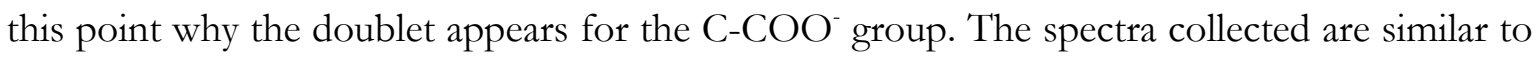
that reported by Moskovits and Suh [13] for mono- and dicarboxylic acids on silver sols, though the peak frequency of the $\mathrm{COO}^{-}$band was comparatively lower by about $20 \mathrm{~cm}^{-1}$. The strength of these three peaks $\left(235,930 / 955\right.$ and $\left.1377 \mathrm{~cm}^{-1}\right)$ indicates that the fatty acids were liberated from the triglyceride structure during sliding, and that it was the fatty acids 
themselves that were chemically adsorbed onto the silver surfaces through their carboxylate groups. This is testament to the view first proposed by Bowden and Moore [21], who observed that lubrication using ethyl stearate on base metals was as effective as stearic acid, hence prompting them to suggest that the esters hydrolysed to form free fatty acids that reacted with the metallic surface.

The appearance of the shoulder at $1700 \mathrm{~cm}^{-1}$, which is synonymous with the stretching of $\mathrm{C}=\mathrm{O}[17-19]$, suggests the presence of non-dissociated carboxylate groups of unadsorbed fatty acids, i.e. carboxylates groups having the $\mathrm{COOH}$ structure as opposed to $\mathrm{COO}^{-}$. For non-dissociated carboxylate groups, it has been reported that the $\mathrm{C}^{-\mathrm{COO}^{-}}$band (normally at approx. $930 \mathrm{~cm}^{-1}$ ) would be red-shifted to $905 \mathrm{~cm}^{-1}$ [17-18] and this can be found in the spectra in the form of a shoulder at the carboxylate band. A similar red shift had also been observed for the $\mathrm{COO}^{-}$band, which might explain the slightly lower than expected peak frequency observed at $1377 \mathrm{~cm}^{-1}$. These are strong indicators that some unadsorbed fatty acids reside at the surface, possibly stacked above the first adsorbed layer.

For the bands assigned to the vibrations of the $\mathrm{CH}_{2}$ and $\mathrm{CH}_{3}$ groups located between 3000 to $2800 \mathrm{~cm}^{-1}$, only a broad band centered at $2930 \mathrm{~cm}^{-1}$, representing the Fermi resonance of the $\mathrm{CH}_{3}$ symmetric and $\mathrm{CH}_{2}$ asymmetric stretching modes, was observed (Fig 6.6A). No peaks were observed at 2850 and $2885 \mathrm{~cm}^{-1}$ which are usually assigned to the symmetric and asymmetric stretching modes of the $\mathrm{CH}_{2}$ group [22-25]. The peak at $760 \mathrm{~cm}^{-1}$ can be assigned to either the terminal $\mathrm{CH}_{3}$ rocking mode [28-29] or $\mathrm{C}-\mathrm{H}$ out of plane deformation [30]. It is interesting to note that there is a lack of contribution from the stretching vibrations of the $\mathrm{CH}_{2}$ groups since the population of $\mathrm{CH}_{2}$ monomers clearly outnumbers the terminal $\mathrm{CH}_{3}$ group for long chain fatty acids. The most plausible explanation for this is that the chains had adopted an all-trans conformation and were aligned at or close to the perpendicular axis of the surface, so that the $\mathrm{C}-\mathrm{H}$ bonds and their stretching axes were essentially parallel to the surface. 
Under SERS selection criteria, no enhancement of such vibrations would occur [26-27]. Interestingly, no peaks could be found near 1060 and $1130 \mathrm{~cm}^{-1}$, which are normally assigned to the vibrations of all-trans segments of alkyl chains [13, 22-25].

The medium intensity peak at $1560 \mathrm{~cm}^{-1}$ peak can also be assigned to the $\mathrm{sp} 2$ bonding of aromatic $\mathrm{C}=\mathrm{C}$ in amorphous carbon structures $[8-12,31]$, while the shoulder at $1630 \mathrm{~cm}^{-1}$ can be assigned to the stretching vibration of the shorter $C=C$ of olefinic chains [16]. These two bands were observed even at the lowest irradiation times of $15 \mathrm{~s}$, which precludes the possibility that this was due to decomposition products due to excessive irradiation.

Two weaker peaks at 1030 and $1167 \mathrm{~cm}^{-1}$ were also noted. Assignment of these two peaks is difficult because they have been associated with several different types of molecular vibrations, including that of the $\mathrm{C}-\mathrm{H}$ in plane bending modes of aromatic compounds [15-16, 32]. The possibility of aromatic compounds cannot be excluded since thermal oxidation of plant oils can produce cyclic and non-cyclic carbon-to-carbon linked dimers and trimers [33].

\subsubsection{Unrefined Canola Oil}

The spectra for the unrefined canola oil exhibit strong peaks at 215, 930 and $1395 \mathrm{~cm}^{-1}$, which have been similarly assigned to $\nu(\mathrm{Ag}-\mathrm{O}), \nu\left(\mathrm{C}_{-} \mathrm{COO}^{-}\right)$and $\nu\left(\mathrm{COO}^{-}\right)$respectively (Fig 6.5B). This indicates that the fatty acids were also liberated from the triglyceride structure to form metallic soaps with the silver surface, as in the case of the refined oil. The $\mathrm{CH}_{2}$ twisting mode near $1295 \mathrm{~cm}^{-1}$ was also observed in the unrefined oil spectra, but with greater intensity. In contrast to the multiple peaks observed in the refined oil spectra from 1500 to $1700 \mathrm{~cm}^{-1}$, only a broad band peaking at $1630 \mathrm{~cm}^{-1}$ (previously assigned to $v(\mathrm{C}=\mathrm{C})$ of olefinic chains) was observed here. Other differences include the disappearance of the weaker peaks at 1030 and $1167 \mathrm{~cm}^{-1}$ found in the spectra of the refined oils. Instead, a medium intensity peak appeared at $1090 \mathrm{~cm}$ ${ }^{1}$ and two shoulders appeared at 892 and $1439 \mathrm{~cm}^{-1}$, where the latter two bands can be 
assigned to the alkyl terminal C-C stretching $[16,25]$ and the $\mathrm{CH}_{2}$ bending and scissoring modes respectively $[22,25]$.

Of notable difference is the shape of the $2800-3000 \mathrm{~cm}^{-1}$ band. In contrast to that of the refined oil, there is an obvious peak near $2850 \mathrm{~cm}^{-1}$ for the SERS spectra of the unrefined oil, which has been assigned to the symmetric stretching of the $\mathrm{CH}_{2}$ group (Fig. 6.6B). This indicates that the boundary film of the unrefined oil was more disordered and less tightly packed, compared to that of the refined oil. The higher intensities of the $1295 \mathrm{~cm}^{-1}$ peak and the appearance of the shoulders at 892 and $1439 \mathrm{~cm}^{-1}$ are also indicative of the increased number of $\mathrm{CH}_{2}$ and $\mathrm{CH}_{3}$ groups that are free to undergo twisting, bending and scissoring motions. It was also noted that the high intensity of the $760 \mathrm{~cm}^{-1}$ peak (assigned to $\mathrm{CH}_{3}$ rocking) of the refined oil spectra was not similarly observed in the unrefined oil spectra, suggesting the lack of alignment of the terminal group as compared to the former. The appearance of a peak near $1090 \mathrm{~cm}^{-1}$ is also in agreement with the shape of the $3000-2800 \mathrm{~cm}^{-1}$ band, since this peak is normally assigned to the gauche conformation of the C-C skeletal backbone - a characteristic typical of highly disordered films [22-23, 25].

The $1090 \mathrm{~cm}^{-1}$ peak can also be associated with the molecular vibrations of phosphates of phospholipids [34-36]. Indeed, ICP-MS of the canola oils revealed that there is five times more phosphorus in the unrefined oil than in the refined oil. The hydrolysis of phospholipids produces lysophospholipids, phosphate anions and amines, in addition to free fatty acids. Regardless of whether this chemical decomposition occurred during sliding, both phosphate anions and phospholipids are highly polar, and would undoubtedly compete with liberated free fatty acids for surface adsorption sites. We believe that this competitive adsorption decreases intermolecular coupling, preventing the fatty acids from forming a tightly packed boundary layer and thus imparting a degree of fluidity in the film. 
The difference in the spectra obtained for refined and unrefined canola oil in the spectral region $1500-1650 \mathrm{~cm}^{-1}$ is also worthy of discussion. Though the $1560 \mathrm{~cm}^{-1}$ peak (assigned to aromatic $\mathrm{C}=\mathrm{C}$ ) was not entirely absent in the unrefined oil spectra, the $1630 \mathrm{~cm}^{-1}$ peak (assigned to olefinic $\mathrm{C}=\mathrm{C}$ ) was always the more dominant in this spectral region. The opposite was true for the refined oil spectra. The higher intensity of the $1630 \mathrm{~cm}^{-1}$ peak of the unrefined oil spectra may at first suggest that there was a greater population of $\mathrm{C}=\mathrm{C}$ from olefinic chains, but a more likely explanation would be that the increased intensity was due to the closer proximity of the double bonds to the silver surface. This supports the view that the chains were in a gauche conformation and were thus oriented closer to the parallel plane of the surface.

From the preceding arguments, it is clear that the tribochemically-reacted films generated by the unrefined canola oil were more disordered than those formed by the refined oil. If this disorder is indeed a characteristic of the unrefined oil boundary films, the mechanism of which this disorder promotes film growth must then be elucidated. We postulate that, while an ordered and tightly packed adsorbed layer possesses higher shear strength and is more wear resistant, their inherently more crystalline and stable nature forbids their interaction with free moving molecules that are constantly being introduced into the contact area during sliding. On the other hand, when the film is sparsely packed, the chains enjoy a greater degree of freedom and interaction with free moving molecules and are hence more susceptible to chemical bonding and intermolecular chain cross-linking (Fig. 6.7). 

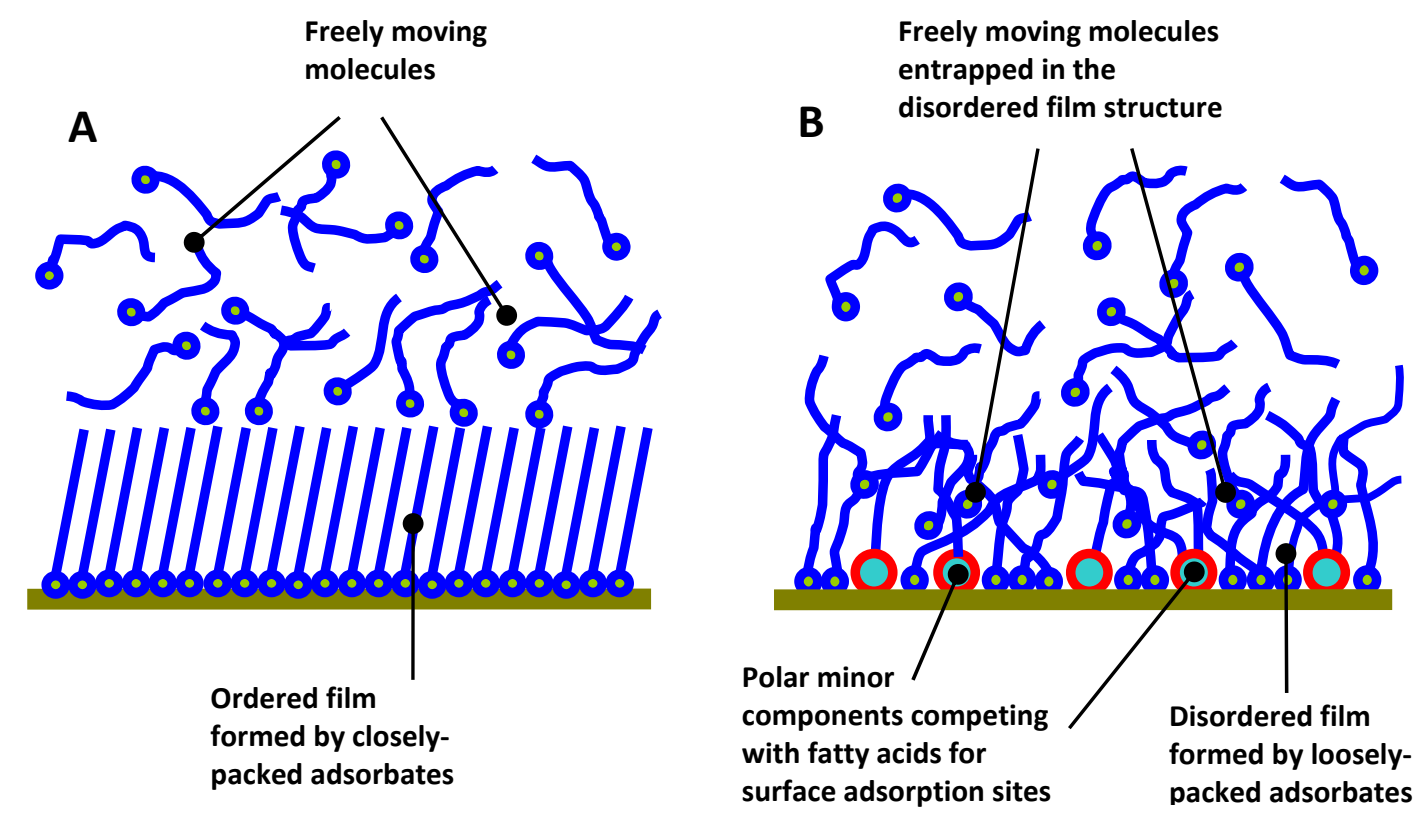

Fig. 6.7: Proposed model of the boundary film structure of A. refined canola oil and; B. unrefined canola oil

\subsection{CONCLUSIONS}

The work presented in this paper has shown that SERS can provide a wealth of information regarding the chemical nature and surface adsorption characteristics of tribochemically-formed boundary films. The analysis clearly showed that for both refined and unrefined canola oils, free fatty acids were liberated from the triglycerides under the action of sliding, and were then adsorbed onto the silver surface via their carboxylate groups. The spectra obtained also revealed that the adsorbed films of the unrefined oil were in a higher state of disorder than that of the refined oil. The more disordered nature of the unrefined oil boundary film could be explained by the competitive adsorption of polar minor components, such as phospholipids, with the free fatty acids, whereby the ability of the free fatty acids to form tightly packed films, would be impeded. It is, however, uncertain whether this disorder in the first adsorbed layer could have been one of the key requirements for film growth as observed under lubricated steel-steel condition. A more conclusive analysis would have to await further experiments using other thin film spectroscopy techniques, such as grazing angle-FTIR or 
sum-frequency generation (SFG) spectroscopy to probe the chemical structure of the thick films as developed under the lubricated steel-steel conditions.

\section{REFERENCES}

1. Moskovits, M.: Surface-enhanced Raman spectroscopy: A brief perspective. In: Kneipp K, Moskovits M, Kneipp H (eds) Surface-enhanced Raman Scattering - Physics and Applications, Topics in Appl. Phys. Springer Verlag, New York, 103, 1-18 (2006)

2. Constantino C.J.L., Lemma T., Antunes P.A., Aroca R.: Single molecule detection using surface-enhanced resonance Raman scattering and Langmuir-Blodgett monolayers. Anal. Chem. 73, 3674-3678 (2001)

3. Kneipp K., Wang Y., Kneipp K., Perelman L.T., Itzkan I., Dasari R.R., Feld M.S.: Single molecule detection using surface-enhanced Raman scattering (SERS). Phys. Rev. Lett. 78(9), $1667-1670$ (1997)

4. Otto A.: What is observed in single molecule SERS, and why? J. Raman Spec. 33, 593-598 (2002)

5. Pieczonka N.P.W., Aroca R.F.: Inherent complexities of trace detection by surfaceenhanced Raman scattering. Chem. Phys. Chem. 6, 2473-2484 (2005)

6. Baeten V., Hourant P., Morales M.T., Aparicio R.: Oil and fat classification by FT-Raman spectroscopy. J. Argri. Food. Chem. 46, 2638-2646 (1998)

7. Muik B., Lendl B., Molina-Díiaz A., Ayora-Cañada M.J.: Direct monitoring of lipid oxidation in edible oils by Fourier transorm Raman spectroscopy. Chem. Phys Lipids 134, $173-182(2005)$

8. Ramsteiner M., Wagner J.: Resonant Raman scattering of hydrogenated amorphous carbon: Evidence for $\pi$-bonded carbon clusters. Appl. Phys. Lett. 51(17), 1355-1357 (1987) 
9. Veres M., Füle M., Tóth S., Koós M., Pócsik I.: Surface enhanced Raman scattering (SERS) investigation of amorphous carbon. Diamond and Related Materials 13, 1412-1415 (2004)

10. Tsang J.C., Demuth J.E., Sanda P.N., Kirtley J.R.: Enhanced Raman scattering from carbon layers on silver. Chem. Phys. Lett. 76(1), 54-57 (1980)

11. Schwan J., Ulrich S., Batori V., Ehrhardt H., Silva S.R.P.: Raman spectroscopy on amorphous carbon films. J. Appl. Phys. 80(1), 440-447 (1996)

12. Gilkes K.W.R., Sands H.S., Batchelder D.N., Robertson J., Milne W.I..: Direct observation of sp3 bonding in tetrahedral amorphous carbon using ultraviolet Raman spectroscopy. Appl. Phys. Lett. 70(15), 1980-1982 (1997)

13. Moskovits M., Suh J.S.: Conformation of mono- and dicarboxylic acids adsorbed on silver surfaces. J. Am. Chem. Soc. 107, 6826-6829 (1985)

14. Miragliotta J., Benson R.C.: Analysis of stearic acid adsorbed on silver surface using optical spectroscopic techniques. In: Advanced Applications of Lasers in Materials Processing/ Broadband Optical Networks/Smart Pixels/ Optical MEMs and Their Applications. IEEE/ LEOS 1996 Summer Topical Meetings. pp 49-50 (1996)

15. Castro J.L., López Ramírez M.R., López Tocón I., Otero J.C.: Surface-enhanced Raman scattering of 3-phenylproponic acid (hydrocinnamic acid). J. Raman Spec, 33, 455-459 (2002)

16. Sengupta A., Thai C.K., Sastry M.S.R., Matthaei J.F., Schwartz D.T., Davis E.J., Baneyx F.: A genetic approach for controlling the binding and orientation of proteins on nanoparticles. Langmuir 24, 2000-2008 (2008)

17. Park S.M., Kim K., Kim M.S.: Adsorption of picolinic and nicotinic acids on a silver sol surface investigated by Raman spectroscopy. J. Mole. Struct. 344, 195-203 (1995)

18. Królikowska A., Kudelski A., Michota A., Bukowska J.: SERS studies on the structure of thioglycolic acid monolayers on silver and gold. Surf. Sci. 532-535, 227-232 (2003) 
19. Han B., Li Z., Wandlowski T.: Adsorption and self-assembly of aromatic carboxylic acids on Au/electrolyte interfaces. Anal Bioanal Chem. 388, 121-129 (2007)

20. Thompson W.R., Pemberton J.E.: Characterization of octadecylsilane and stearic acid layers on $\mathrm{Al}_{2} \mathrm{O}_{3}$ surfaces by Raman spectroscopy. Langmuir 11, 1720-1725 (1995)

21. Bowden F.P., Moore A.C.: Physical and chemical adsorption of long chain compounds on radioactive metals. Trans. Faraday Soc. 47, 900-908 (1951)

22. Orendorff C.J., Ducey M.W. Jr., Pemberton J.E.: Quantitive correlation of Raman spectral indicators in determining conformational order in alkyl chains. J. Phys. Chem. A 106, 6991$6998(2002)$

23. Snyder R.G., Strauss H.L.: C-H Stretching Modes and the Structure of n-Alkyl Chains. 1. Long, Disordered Chains. J. Phys. Chem. 86, 5145-5150 (1982)

24. Macphail R.A., Strauss H.L., Snyder R.G.: C-H stretching modes and the structure of nalkyl chains. 2. Long, all-trans chains. J. Phys. Chem. 88, 334-341 (1984)

25. Brown K.G., Bicknell-Brown E., Ladjadj M.: Raman-active bands sensitive to motion and conformation at the chain termini and backbones of alkanes and lipids. J. Phys Chem 91, $3436-3442(1987)$

26. Philip D., John A., Panicker C.Y., Varghese H.T.: FT-Raman, FT-IR and surface enhanced Raman scattering spectra of sodium salicylate. Spectrochim. Acta, Part A 57, 1561-1566 (2001)

27. Suh J.S., Michaelian K.H.: Surface-enhanced Raman scattering as a probe of surface geometry effects on the polymerization of acrylic acid on silver. J. Phys. Chem. 91, 598-600 (1987) 
28. Overman S.A., Thomas G.J. Jr.: Raman markers of nonaromatic side chains in an $\alpha$-helix assembly : Ala, Asp, Glu, Gly, Ile, Leu, Lys, Ser, and Val residues of phage fd subunits. Biochemistry 38, 4018-4027 (1999)

29. Matsuura H., Fukuhara K., Masatoki S., Sakakibara M.: Molecular conformation of nonionic surfactant in the solid state. A Raman spectroscopic study of a homologous series of $\alpha$-n-alkyl- $\omega$-hydroxyoligo(oxyethylene)s. J. Am. Chem. Soc. 113, 1193-1202 (1991)

30. Xue G., Dong J., Zhang J.: Surface-enhanced Raman scattering of polymer on metal. 2. Molecular chain orientation of polybenzimidazole and poly $(\mathrm{L}-$ histidine $)$ and its transition. Macromolecules 24, 4195-4198 (1991)

31. Pagannone M., Fornari B., Mattei G.: Molecular structure and orientation of chemisorbed aromatic carboxylic acids: surface enhanced Raman spectrum of benzoic acid adsorbed on silver sol. Spectrochim. Acta, Part A, 43(5), 621-625 (1987)

32. Wei F., Zhang D., Halas N.J., Hartgerink J.D.: Aromatic amino acids providing characteristic motifs in the Raman and SERS spectroscopy of peptides. J. Phys. Chem. B 112 9158-9164 (2008)

33. Choe E., Min D.B.: Mechanisms and Factors for Edible Oil Oxidation. Comp. Rev. Food Sci. \& Food Safety. 5(4), 169-186 (2006)

34. Niaura G., Gaigalas A.K., Vilker V.L.: Surface-enhanced Raman spectroscopy of phosphate anions: adsorption on silver, gold and copper electrodes. J. Phys. Chem. B 101, $9250-9262(1997)$

35. Meier R.J., Csiszár A., Klumpp E.: On the interpretation of the $1100 \mathrm{~cm}^{-1}$ Raman band in phospholipids and other alkyl-containing molecular entities. J. Phys Chem B 110, 5842-5844 (2006) 
36. Bunding Lee K.A.: Fourier transform infrared spectroscopic studies of microstructures formed from 1,2-bis(10, 12-tricosadiynoyl)-sn-glycero-3-phosphocholine. J. Phys. Chem. 93, 926-931 (1989) 


\section{Conclusions}

The development of the new tribometer has revealed new tribological phenomena of plant oils previously not observed before. These observations provide strong evidence that the boundary film thicknesses of plant oils are not a constant, but a dynamic property of the plant oil itself, which is dependant on many factors. Attempts have been made to correlate the physical properties, free fatty acid content, fatty acid composition and elemental composition of each plant oil tested to the growth of their boundary films. However, none of the properties thus far have provided a strong correlation and the mechanism of the film's growth remain elusive. Although results from SERS and ICP-MS analysis seem to suggest that the presence of phosphorus, could be the major contributor to the observed increase in film thickness, this remains to be further investigated. The observations and deductions from the research work presented in this thesis are summarized in this chapter.

\subsection{SUMMARY OF KEY FINDINGS}

\subsubsection{Stage 1}

In the first stage, a new tribometer, employing the use of the capacitance method, was developed to measure the boundary film thickness of plant oils, simultaneously with friction and wear. The calculation method to convert capacitance values to film thickness values was formulated using fundamental principles of geometry and capacitance. The capacitance 
required the dielectric constants at ultra-high pressure of the oils tested to be known. This required the use of a high pressure condenser to experimentally determine those values. Due to the high cost of manufacturing such a device, it was decided that empirical methods are used for the prediction of the dielectric constant at the contact pressure.

The use of a precise and accurate LCR method for capacitance measurement, along with properly shielded probes and electronics enabled the noise-to-signal ratio to be kept low. It was also found that hard steels of $800 \mathrm{HV}$ or more, polished to a mirror finish, was essential to obtain stable capacitance readings and high reproducibility of results. The smooth surfaces also allowed the frictional and film thickness changes due to tribochemical reaction to be observed more gradually. Such conditions were necessary for stable readings to be obtained, while maintaining objectivity in the experiments.

It was observed that there was greater variability of the capacitance readings, when films were very thin (typically less than $6 \mathrm{~nm}$ ) due to the high sensitivity of the capacitance method in this film thickness range. This can also be attributed to the entrapment of metallic particles in the contact area and slight variations in the topography and surface roughness along the wear track. These variations were minimized by signal processing, i.e. averaging the capacitance readings over one revolution of the disc.

The pilot tests indicate that the capacitance method can be applied convincingly to study boundary lubrication in situ. Our pilot long term sliding tests conducted on sunflower oil using the new method clearly showed an appreciable growth in the boundary film thickness up to $8 \mathrm{~nm}$, with a corresponding drop in the coefficient of friction. There was almost no wear during the observed film growth. The size of the triglyceride molecule suggests that the thickness of two opposing boundary films should not exceed more than $4 \mathrm{~nm}$. These results clearly prove the hypothesis that organic boundary films of plant oils do not remain constant 
over long sliding periods. While the concept of film growth by tribochemical reaction may not be new, this is the first time that it has been observed in situ and in plant oils.

\subsubsection{Stage 2}

In the second stage, the experiment was extended to 12 other plant oils. In all, 4 refined and 9 unrefined oils were tested. In an effort to correlate the relationship between the film growth behaviour with the physical, optical and dielectric properties (density, viscosity, refractive index, dielectric constant), the fatty acid composition and the amount of free fatty acid (FFA) of the fresh oils, were measured.

Some of the oils exhibited surprisingly high levels of film growth. For example, the boundary film of unrefined canola oil grew to a thickness in excess of $100 \mathrm{~nm}$ after 18 hours of sliding. This is followed by sesame oil, sunflower oil and macadamia oil, which exhibited final film thicknesses of approximately, 52, 39, and $29 \mathrm{~nm}$ respectively. These four oils were all unrefined oils. The boundary films of the other plant oils generally experienced a slight increase in the film thickness, except for unrefined safflower oil, which collapsed after 400 min of sliding, and extra virgin olive oil, which showed no change in the film thickness after 18 hours. The results in this work convincingly show that the long term film thicknesses may change over long sliding periods and these changes are unique to each oil tested. The experiments were repeated 3 times to ensure that the results are reproducible. The frictional traces obtained during the experiments were also found to vary with the thickness of the film, presumably due to a change in the shear strength of the film.

It was initially expected that the growth of a plant oil boundary film might have been related to the degree of unsaturation in the oil, since polyunsaturated oils would be theoretically more unstable than monounsaturated oils. Analyses of the results, however, revealed otherwise and showed no correlation between the fatty acid compositions and the 
film thicknesses observed. It prompted the suggestion that minor components in the unrefined oils might have been a key ingredient for its ability to grow to such thick films. ICPMS analysis was conducted on 10 of the tested oils after this series of experiments. The results seem to indicate that plant oils, which exhibited high film growth, seem to contain a higher level of phosphorus, presumably from phospholipids.

During the course of these experiments, the films were also observed to exhibit some viscoelastic behaviour under stop conditions. Such characteristics seems to reinforce the notion that there is a tenaciously thick boundary film that are highly viscous and are able to separate the surfaces even when the lubricated surfaces are not in relative motion. Further tests also indicate that the films formed appear to exhibit transient behaviours upon changes in sliding speed.

\subsubsection{Stage 3}

In the third and final phase of this work, surface-enhanced Raman spectroscopy (SERS) was employed to study and compare the differences in chemical composition of surface adsorbates between refined and unrefined canola oil. The SERS method was selected because of its high sensitivity to the first adsorbed boundary layers. It also provides information on chemical bonding, molecular orientation and the degree of packing order. This is the first time that SERS has been used to study tribochemically-formed boundary films in the field of both Tribology and Raman spectroscopy. The results revealed strong evidence that fatty acids were liberated from the triglyceride structure during sliding and chemically adsorbed onto the wear surface of the silver-coated bearing ball. There is also strong evidence that the films of the unrefined canola oil were more disordered than the refined oil.

It is postulated that the ability of free fatty acids to chemically react with stably ordered films would have been quite limited, since the latter are tightly bound to the surface 
and are closely packed. With disordered films, however, the double bonds are more likely to be exposed, while the looseness of packing allows free fatty acids to be entrapped between the chains of adsorbed fatty acid. Coupled with the presence of high pressure and shear, it is possible that a thermodynamically unstable system would have been induced, promoting chemical bonding and a chain reaction that leads to polymerization and ultimately, film growth.

ICP-MS analysis conducted earlier in Stage 2 revealed higher traces of phosphorus in the unrefined canola oil and macadamia oil, both of which exhibited high film growth. Phospholipids have high adsorption strength to steel surfaces and are capable of displacing triglycerides from their adsorption sites. However, evidence of phosphorus or phospholipids in the bulk oil itself is not adequate proof of its involvement in the tribochemical process. Although the appearance of the $1090 \mathrm{~cm}^{-1}$ peak in the Raman spectra for unrefined canola oil seemed to suggest that phosphates could be present in the tribochemically formed boundary films, this remains inconclusive, since the peak can also be associated with the skeletal C-C vibrations of alkyl chains in a gauche conformation. Confirmation of the presence and influence of phosphates in the tribochemical process must await further work via other surface-sensitive spectroscopy techniques, such as grazing-angle FTIR.

The SERS analysis prompted further research questions that could not be answered at this stage - What caused the disorder in the film structure? Could the presence of phospholipids have caused the disorder in the film structure and thus, indirectly facilitated the film growth, or could they have been directly part of the chain of chemical reactions in the polymerization process? 


\subsection{RECOMMENDATIONS FOR FUTURE WORK}

\subsubsection{Effects of Varying Sliding Speeds on the Film Thickness and Friction}

In Section 5.4.5, it was shown that there is a transitional phase in the film thickness and friction, when sliding speeds were altered quickly. This behaviour for tribochemically formed films has been observed for the first time through the use of the new tribometer. To maintain objectivity of the experiments in Chapter 5, it was decided that this phenomenon would be tested at a later stage. A further investigation under such conditions should be conducted to ascertain that these observations are not due to errors caused by temperature fluctuations affected by the change in the sliding speed. Other instruments such as optical interferometry methods can be employed on tribochemically-formed films to confirm this transitional change. Future tests could also be conducted on other oils, including mineral oil based lubricants, with different molecular structures. This behaviour may further explain the physical and chemical nature of the films, and provide an insight to the anti-wear mechanisms of plant oil boundary films.

\subsubsection{Quantification of Minor Components and Tribological Test}

As mentioned in the summary of key findings, the mechanisms of plant oil boundary film growth have not been determined. Although GCMS and ICP-MS have been employed in the determination of the fatty acid composition and the elemental composition respectively, there are many other minor components that have not been identified and quantified in the experiments presented in Chapter 5, due to cost and time constraints. Although it has been postulated that phospholipids may be the cause of the growth, more objective experiments will need to be conducted to validate this hypothesis, and if found true, the mechanisms in which phospholipids influence the tribochemcal processes must then be investigated. 
A more detailed chemical compositional study should be made of the plant oils before they are being tested tribologically. The compositional breakdown should include other minor components such as, trace metals, peroxides, water, phospholipids, tocopherols, sterols, phenolic compounds and carotenoids. This can be accomplished by the use of highperformance liquid chromatography (HPLC) techniques. Due to minor differences in the fatty acid composition and the quantity of minor components from a different cultivation batch of the plant oils, it is essential that the experiments in Chapter 5 be repeated for the oils tested to maintain objectivity, otherwise correlation between the film growth and minor components would not be conclusive. Statistical correlation between the minor components and the film growth could then used to identify key components that may be responsible for the tribochemical reaction.

\subsubsection{Validation of the Effects of Minor Components in Film Growth}

With the minor components identified, tribological experiments in Chapter 5 should then be repeated to validate the statistical correlation results. Pure triglycerides blended with selected minor components in varying degrees should be used, and compared against pure triglycerides as the control test fluid. A comparison between monounsaturated triglycerides doped with a minor component, against a pure saturated triglyceride doped with the same minor component may also provide insight to the role of the double bond in the tribochemical reaction with the double bond.

The use of pure compounds eliminates uncertainties associated with possible interference from other constituents that may affect the tribochemical process. For example, if the effects of phospholipids are to be tested, pure compounds such as, phosphatidic acid (PA), phosphatidylethanolamine (PE) and phosphatidylcholine (PC) could be used. This also allows the tribochemical process to be modeled and then verified by surface spectroscopic techniques, such as SERS or RAIRS. As experienced in the work presented in Chapter 6, 
knowledge of the constituents before and after sliding tests would significantly help the assignment of Raman peaks.

\subsubsection{Surface-sensitive Spectroscopy Analysis}

As mentioned in Chapter 6, the SERS technique is constraint by the fact that the surface of the substrate must be made of silver, gold or copper for it to work. This differs from the tribological surfaces used in Chapter 5, where the boundary films of unrefined canola oil have been observed to grow more than 25 times its initial thickness on hardened 52100 steel. A more conclusive analysis must therefore await the use of other surface-sensitive spectroscopic techniques, such as grazing angle-FTIR and sum-frequency generation (SFG) spectroscopy., where analysis can be conducted on the same sample where the film growth has been observed. These are complementary methods to SERS and should provide more information regarding the chemical nature of the tribofilms formed. Both the compositional determination of the bulk oil and the spectroscopic study of the boundary films would be essential to unlocking the secrets of plant oil boundary film growth. 
Appendix A: Original Film Thickness and Friction Traces 
SUNFLOWER (REFINED)
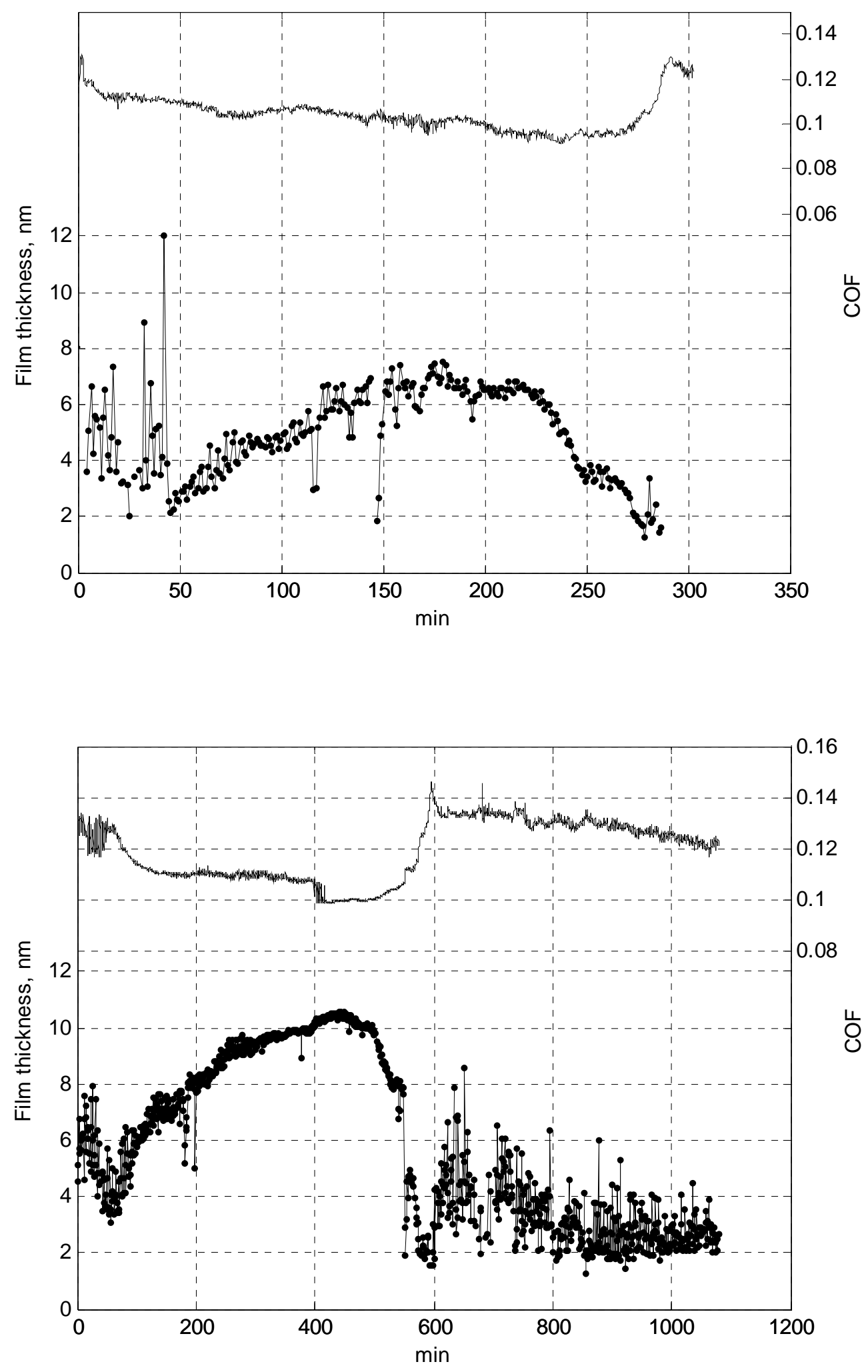


\section{PALM OLEIN (REFINED)}
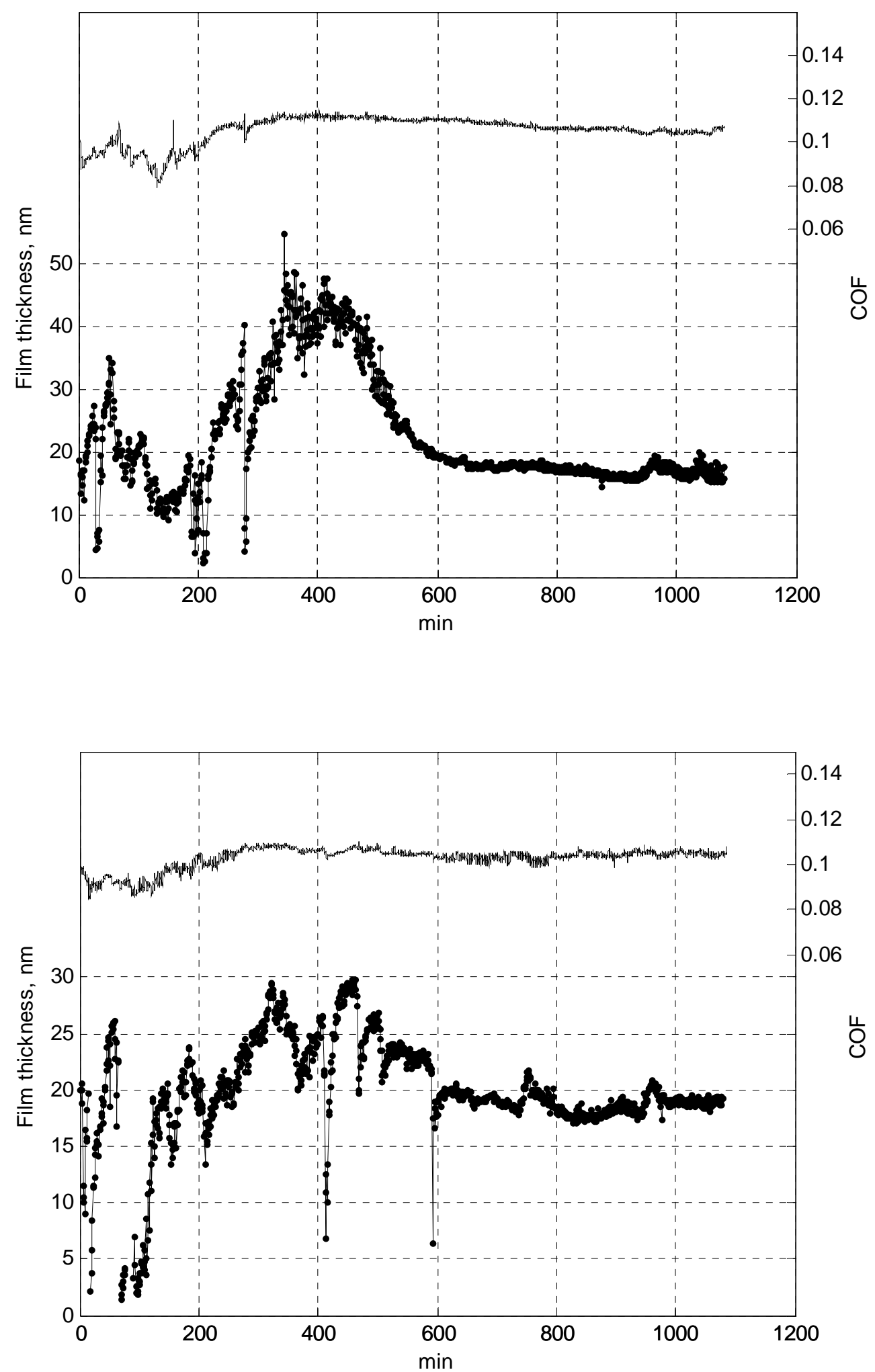
HIGH OLEIC SUNFLOWER (REFINED)
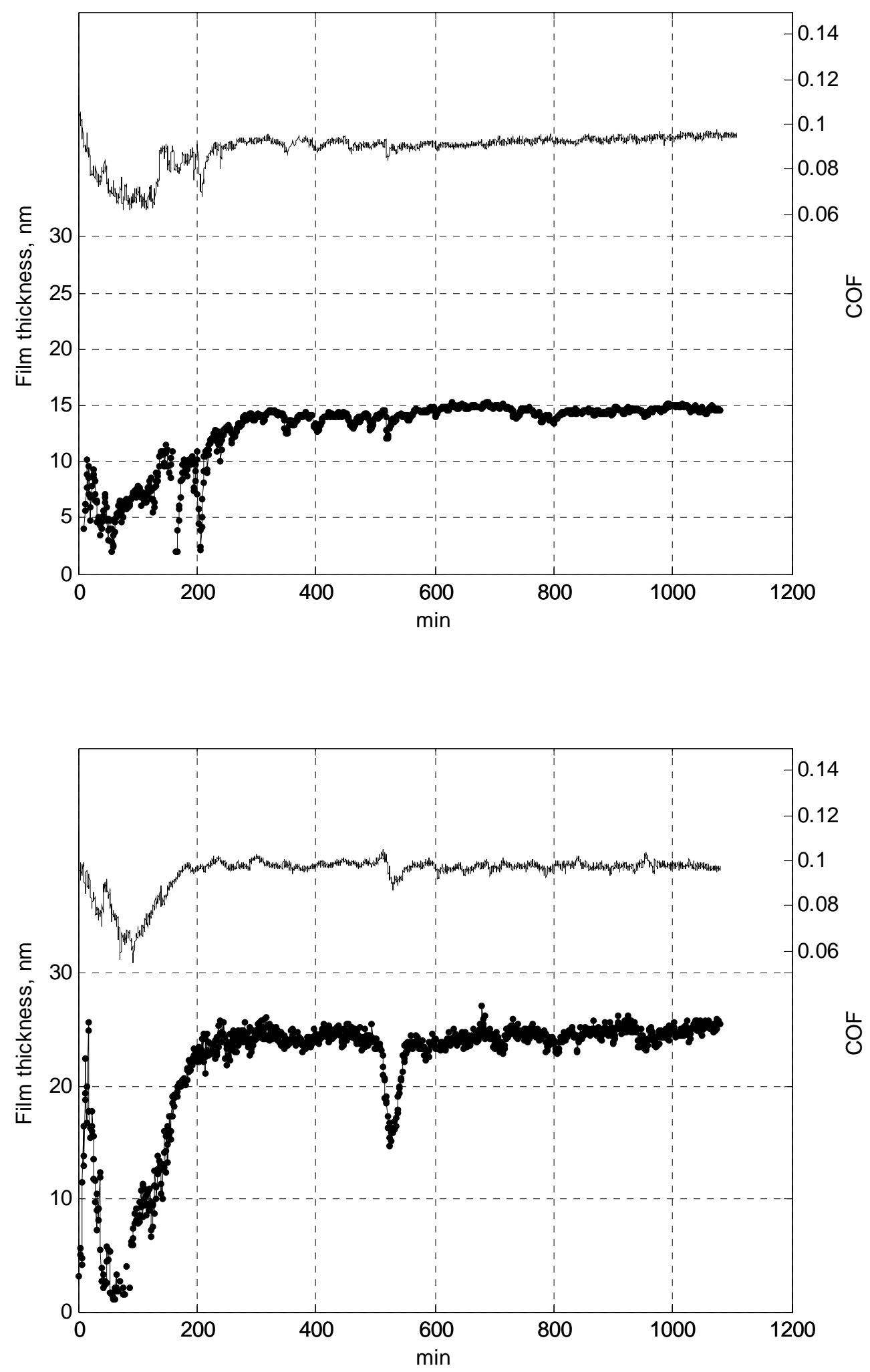


\section{CANOLA (REFINED)}
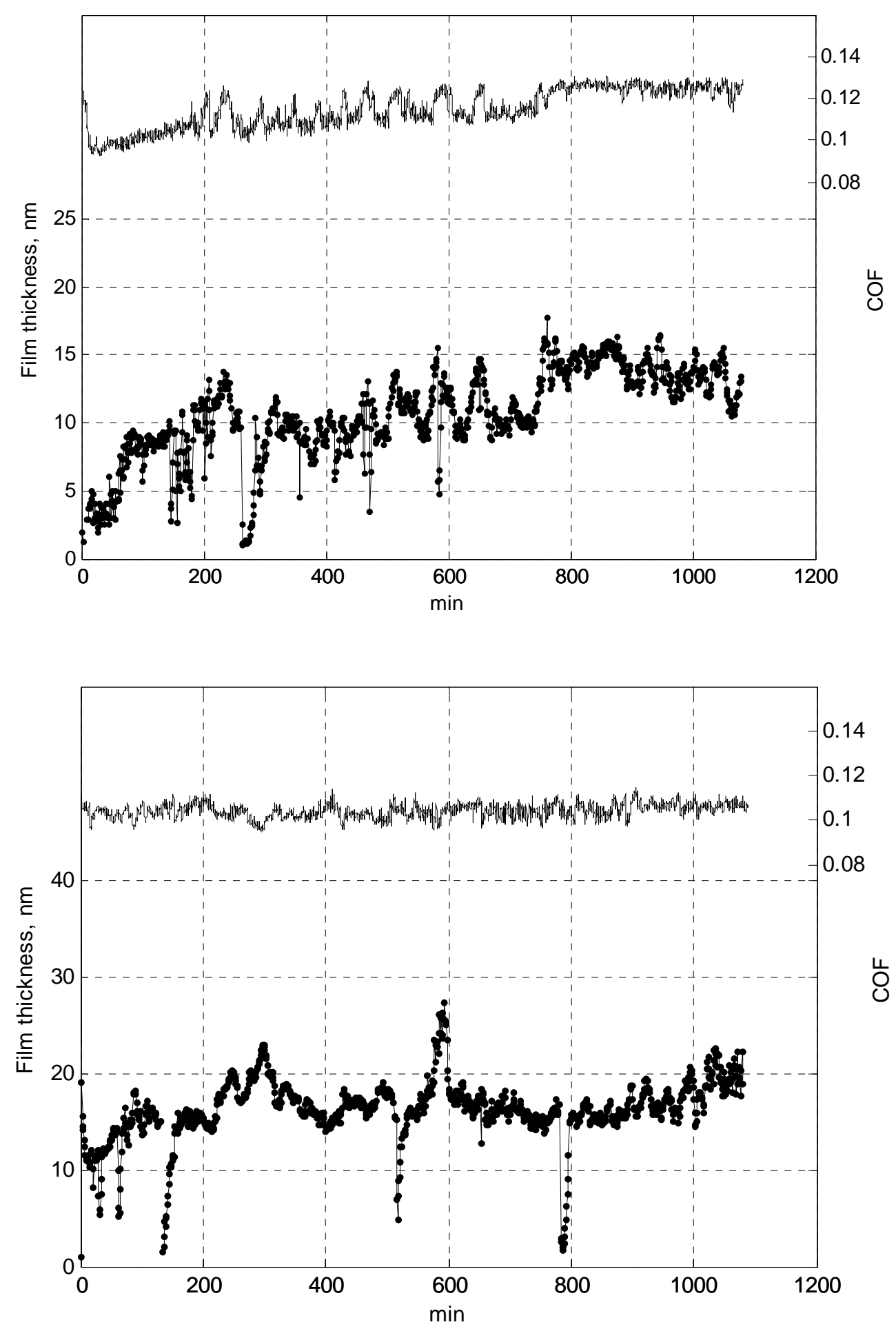


\section{WHEATGERM (UNREFINED)}
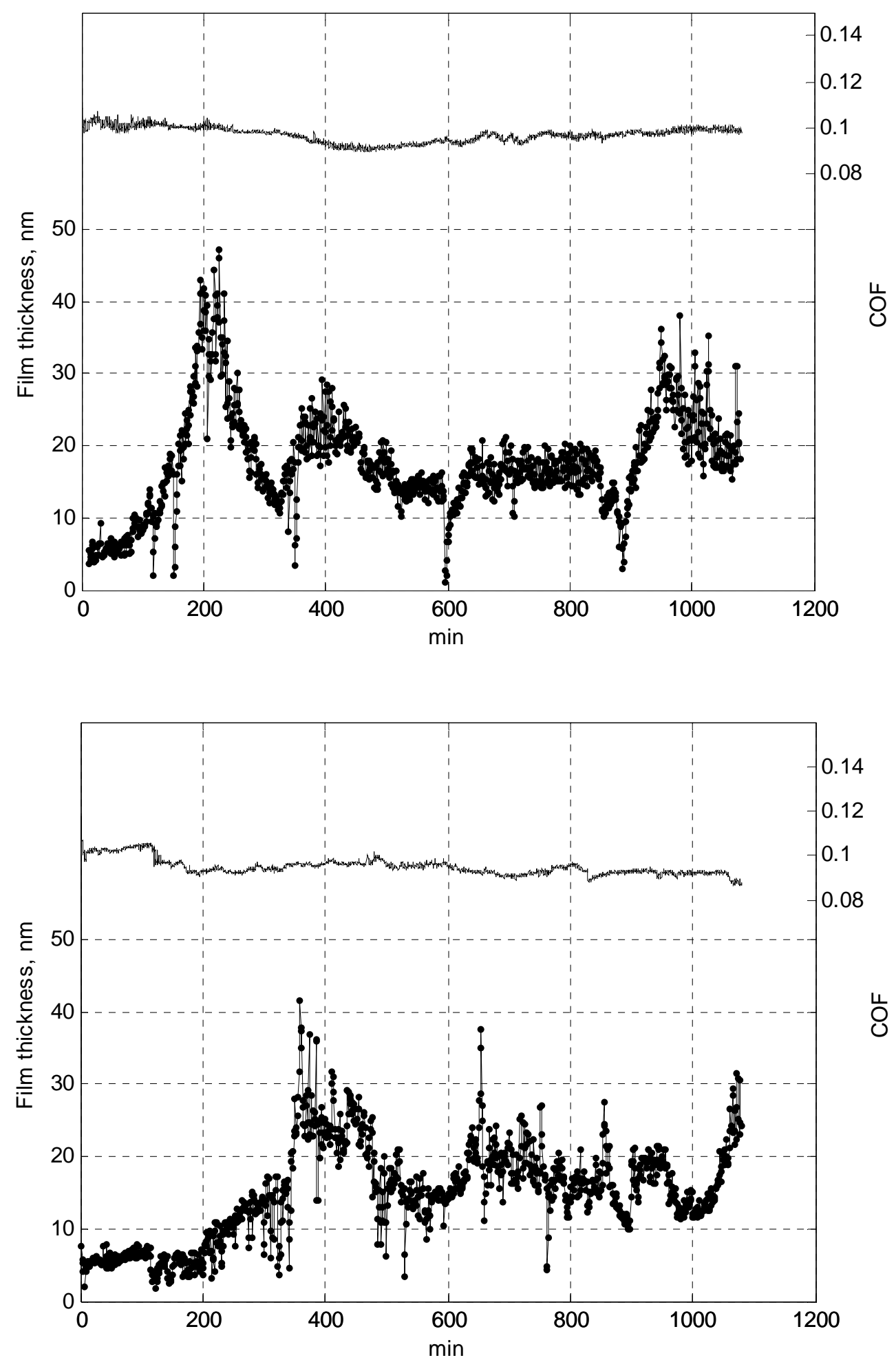
SESAME (UNREFINED)
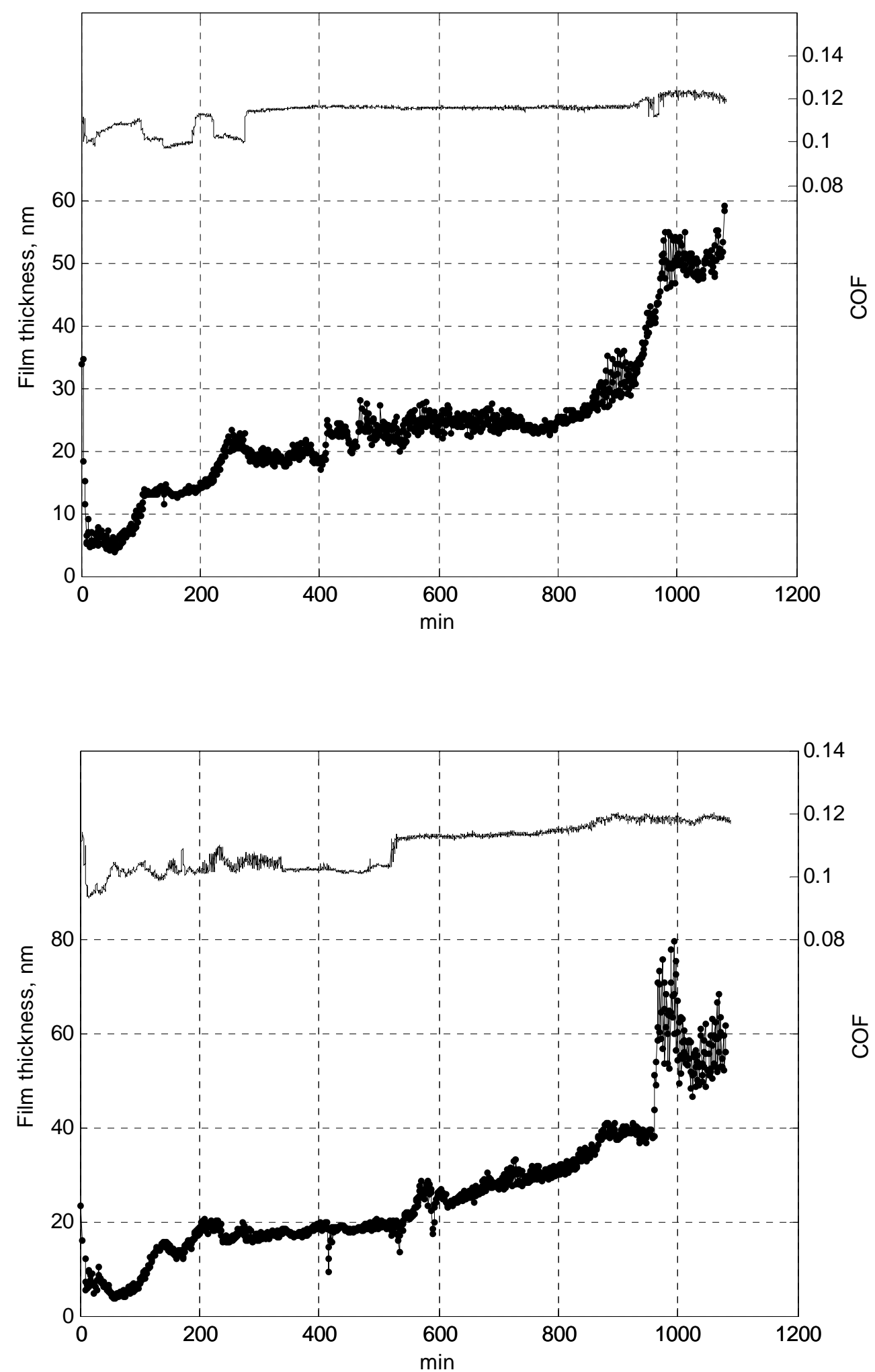


\section{SAFFLOWER (UNREFINED)}
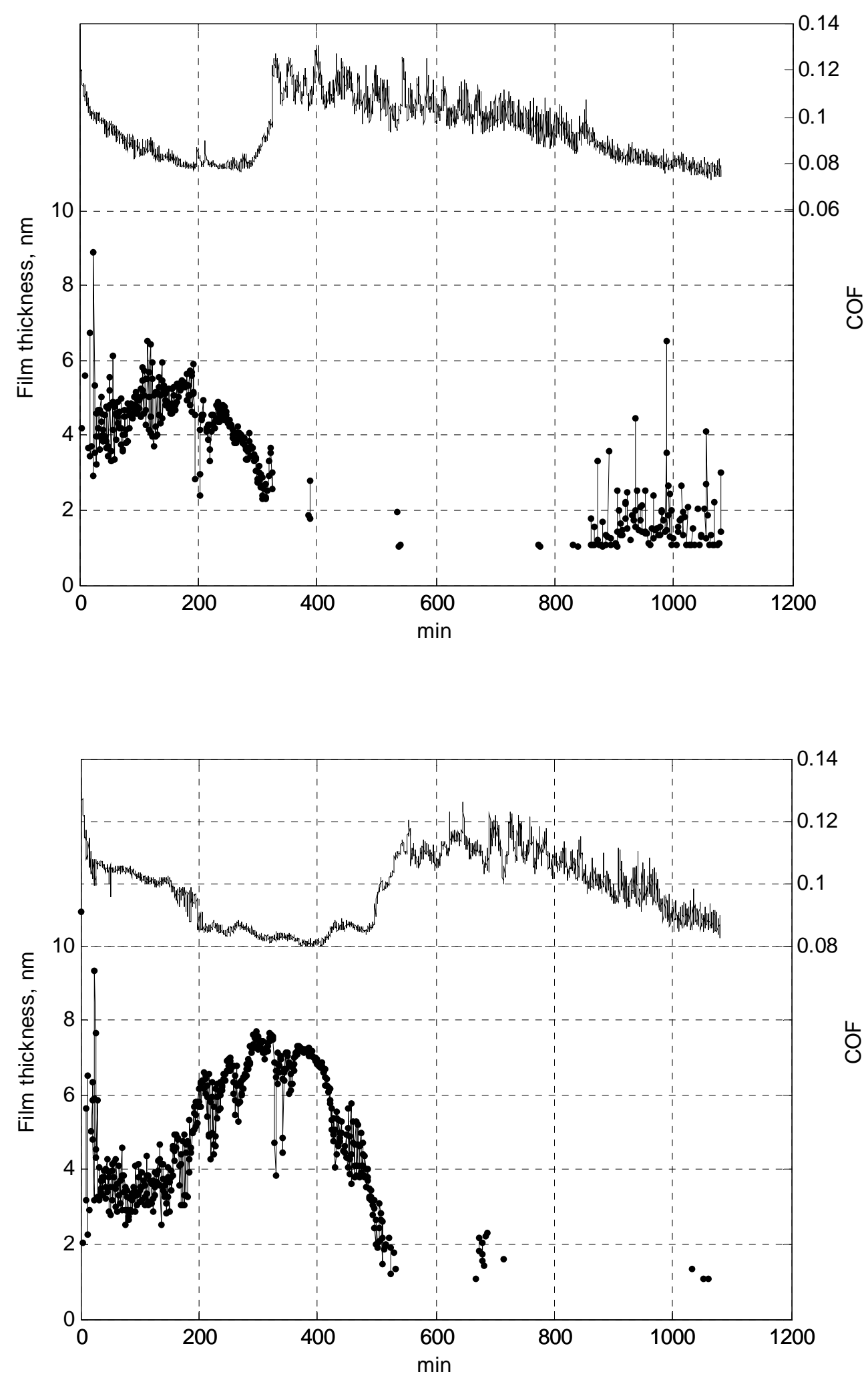
MACADAMIA (UNREFINED)
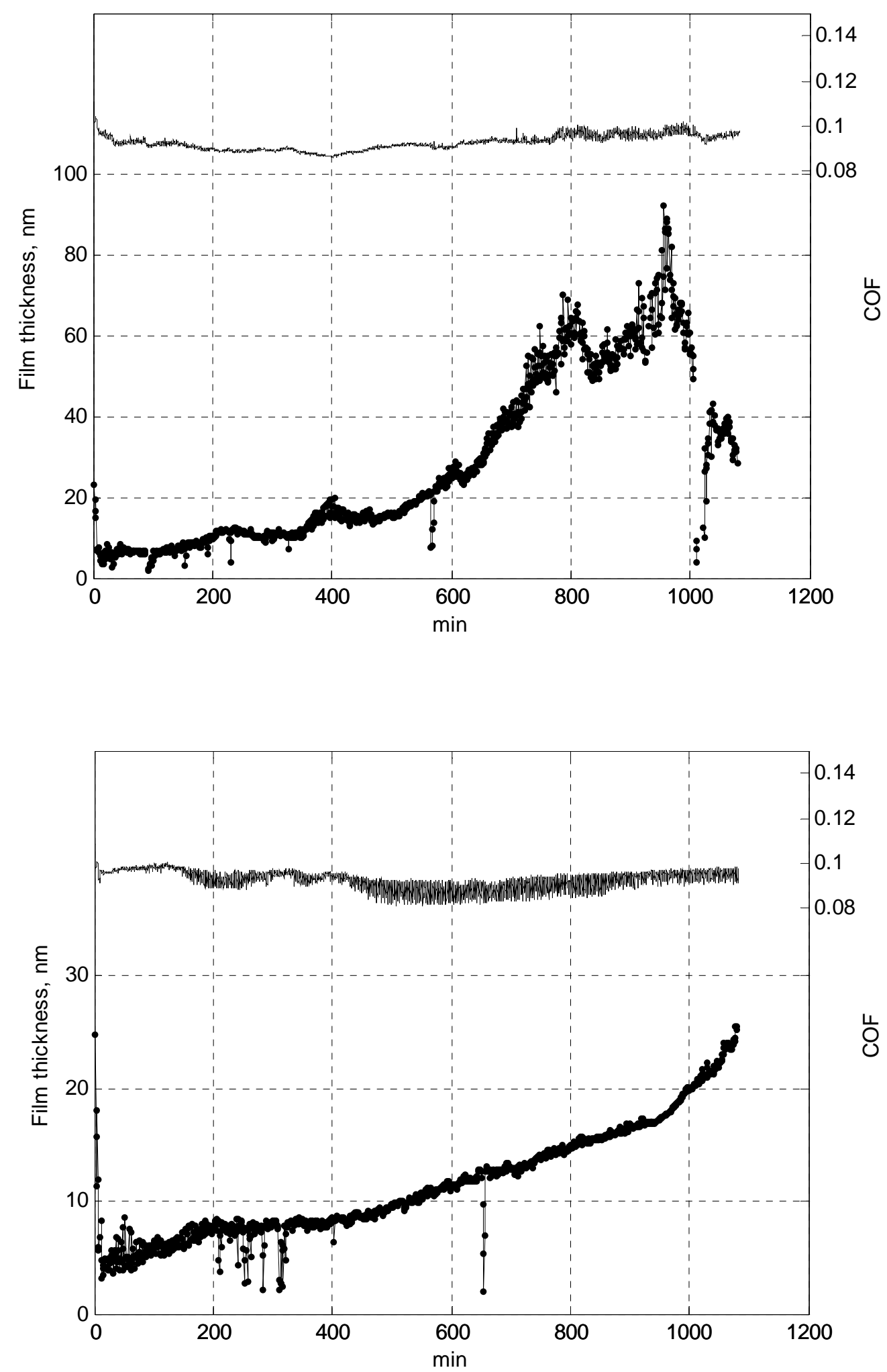
HIGH OLEIC SUNFLOWER (UNREFINED)
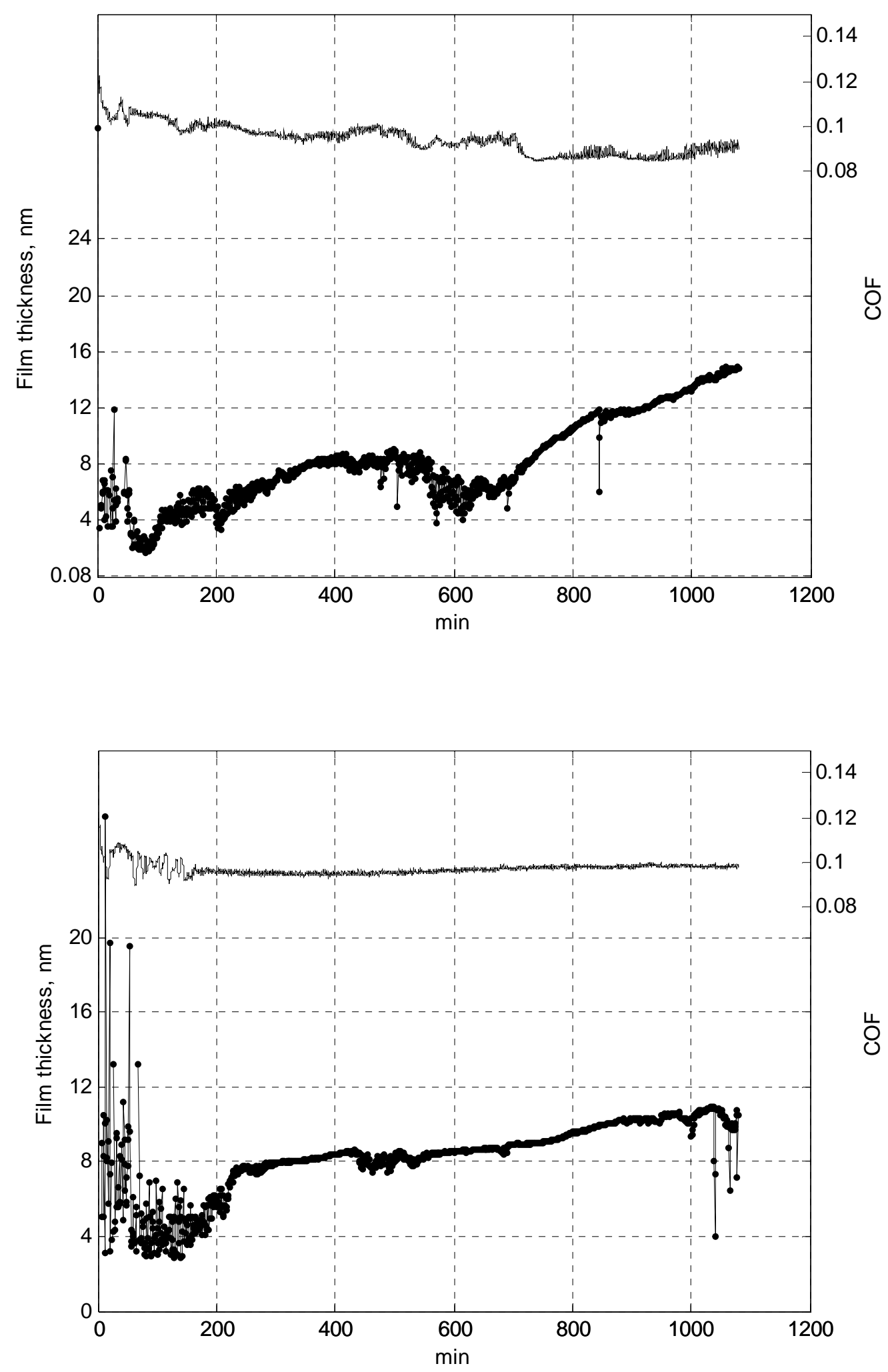
EXTRA VIRGIN OLIVE (UNREFINED)
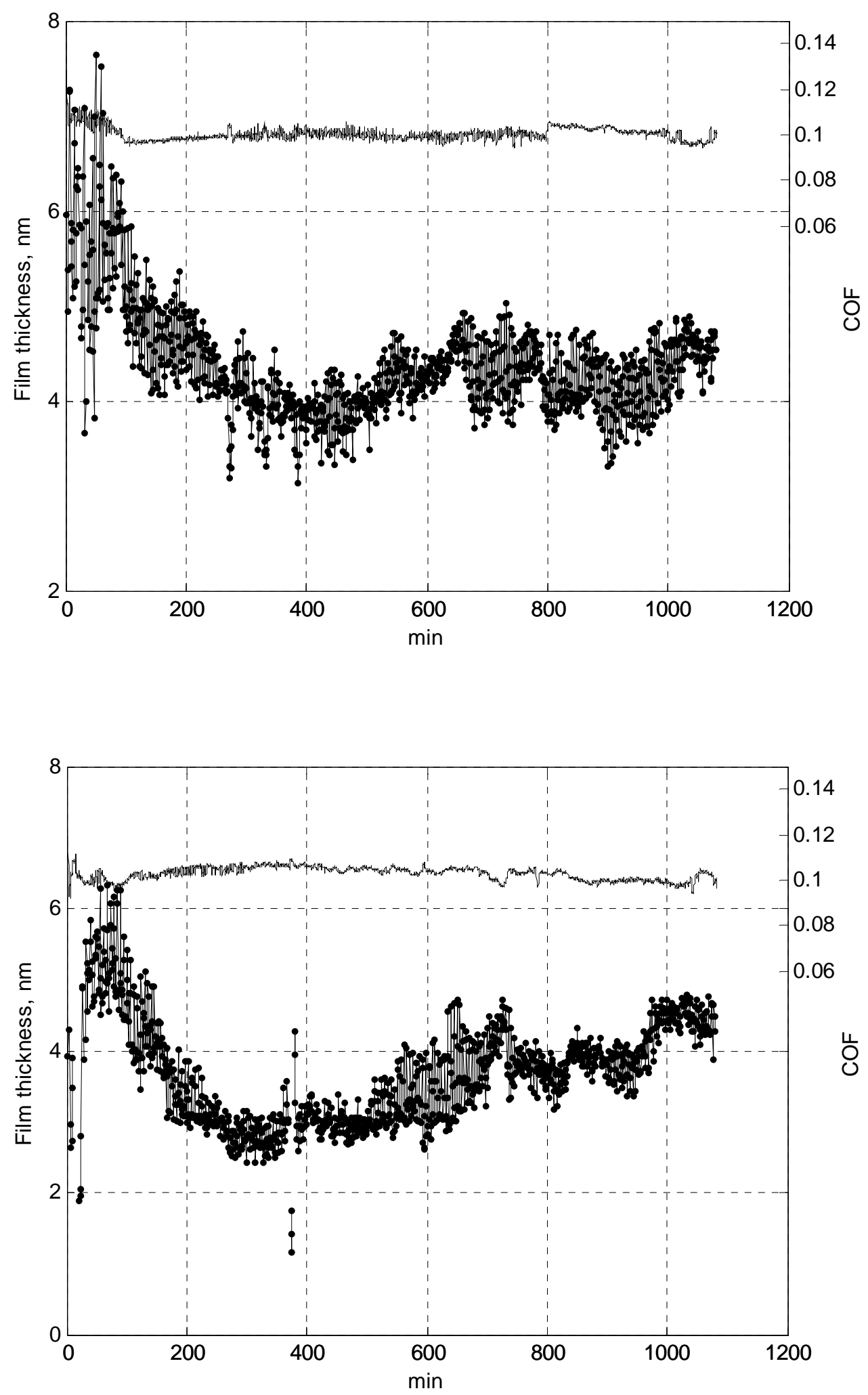


\section{CANOLA (UNREFINED)}
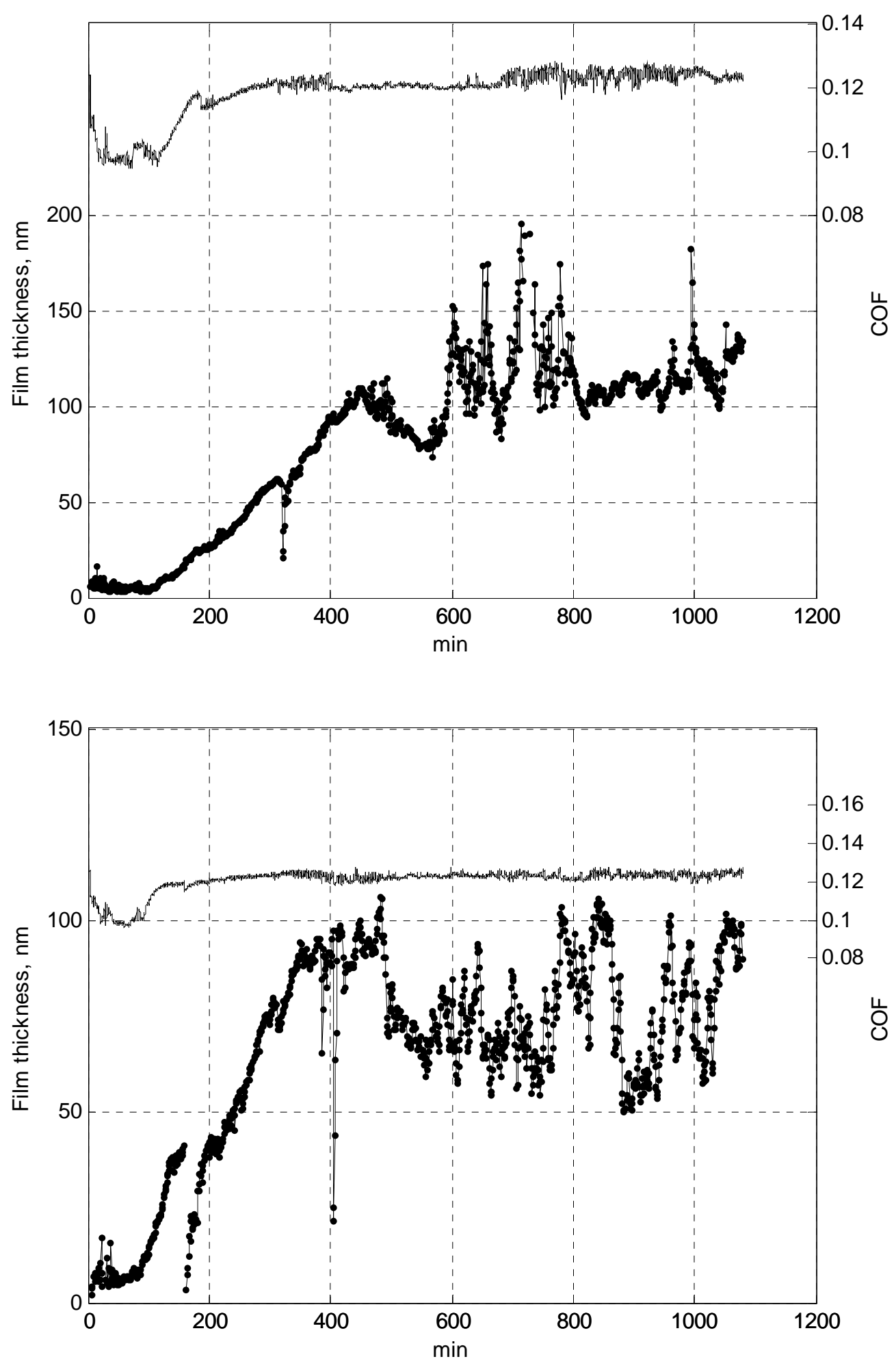
FLAXSEED (UNREFINED)
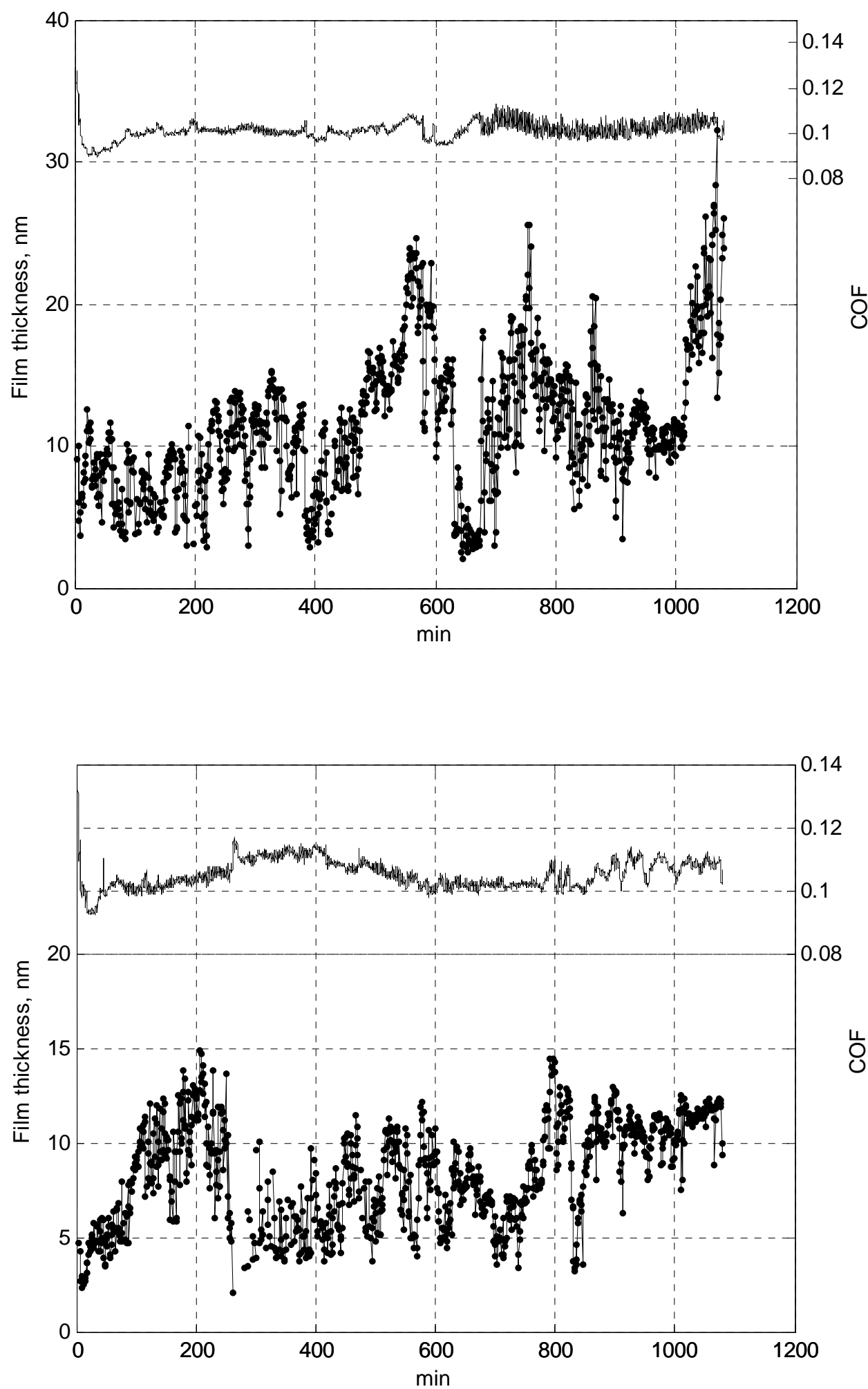
Appendix B: Publications 


\title{
The Study of the Dynamic Thickness of Organic Boundary Films Under Metallic Sliding Contact
}

\author{
Wen Hsi Chua · Gwidon W. Stachowiak
}

Received: 15 December 2009/ Accepted: 18 May 2010

(C) Springer Science+Business Media, LLC 2010

\begin{abstract}
The paper herein proposes a method to simultaneously measure the friction, wear, and thickness of boundary films under a pure sliding contact between metallic surfaces. The method is based on the capacitance method used by Crook and Dyson et al. to measure elastohydrodynamic film thicknesses. Pilot tests conducted on sunflower oil using the new method showed an appreciable growth in the boundary film thickness, with a corresponding drop in the coefficient of friction. Very little wear was observed during the film growth. It is postulated that this growth was due to the polymerization of the unsaturated fatty acid chains.
\end{abstract}

Keywords Vegetable oils .

Boundary lubrication test methods

\section{List of Symbols}

$A_{\text {a }} \quad$ Apparent area of contact

$A_{\mathrm{nr}} \quad$ Apparent area of contact less the real areas of contact

$A_{\mathrm{r}} \quad$ Real area of contact. For $A_{\mathrm{r}} / A_{\mathrm{a}}=1, A_{\mathrm{r}}=$ $\pi\left(\frac{3 W R_{\mathrm{b}}}{2 E^{\prime}}\right)^{2 / 3}$

$C_{\mathrm{b}} \quad$ Capacitive contribution outside the apparent area of contact
$C_{\mathrm{nr}} \quad$ Capacitive contribution of $A_{\mathrm{nr}}$

$C_{\text {air }} \quad$ Reference capacitance measured in air

$C_{\mathrm{r}} \quad$ Capacitive contribution of $A_{\mathrm{r}}$

$C_{\mathrm{t}} \quad$ Total capacitance $=C_{\mathrm{b}}+C_{\mathrm{nr}}+C_{\mathrm{r}}$

$E_{1}, E_{2} \quad$ Elastic modulus of the rubbing solids

$E^{\prime} \quad$ Reduced Young's modulus of elasticity where, $\frac{1}{E^{\prime}}=\frac{1}{2}\left(\frac{1-v_{1}^{2}}{E_{1}}+\frac{1-v_{2}^{2}}{E_{2}}\right)$

$n \quad$ Refractive index

$p \quad$ Pressure (in MPa)

$p_{\mathrm{o}} \quad$ Atmospheric pressure, $0.1 \mathrm{MPa}$

$p_{\mathrm{a}} \quad$ Mean apparent contact pressure

$r \quad$ Radial coordinates of the contact

$R_{\mathrm{a}} \quad$ Centre-line average of the surface roughness

$R_{\mathrm{b}} \quad$ Radius of the ball

$R_{\mathrm{h}} \quad$ Radius of the Hertzian contact area

$R_{\mathrm{w}} \quad$ Radius of the ball wear scar

$z \quad$ Axial coordinates of the contact

$z_{\mathrm{d}} \quad$ Maximum deflection of the contact (at $r=0$ ), $R_{\mathrm{b}}-\sqrt{R_{\mathrm{b}}^{2}-R_{\mathrm{h}}^{2}}$

$z_{\mathrm{r}} \quad$ Minimum film thickness of the contact

$z_{\mathrm{w}} \quad$ Wear depth of the ball, $R_{\mathrm{b}}-\sqrt{R_{\mathrm{b}}^{2}-R_{\mathrm{w}}^{2}}$

$\varepsilon_{\text {air }}$

$\varepsilon_{\mathrm{b}}$

Dielectric constant of air, taken as 1.00059

Dielectric constant of the bulk lubricant

Permittivity of free space

Dielectric constant of the boundary layer at

Hertzian pressure

Dielectric constant at high frequencies,

approximated by $n^{2}$

Dynamic viscosity

$\eta$

$\kappa_{\mathrm{s}} \quad$ Mean curvature of the surface profile

$\rho \quad$ Density at the pressure of interest

$\rho_{\mathrm{o}} \quad$ Density at atmospheric pressure and test temperature

W. H. Chua $(\varangle)$ · G. W. Stachowiak

University of Western Australia, Nedlands, WA, Australia

e-mail: whchua@mech.uwa.edu.au $\sigma \quad$ Standard deviation of surface roughness

$v_{1}, v_{2} \quad$ Poisson's Ratio of the rubbing solids 


\section{Introduction}

Despite numerous studies on boundary lubrication since the classical work by Bowden and Tabor [1], our understanding of boundary films is still limited at their initial state of adsorption or chemical reaction with the surface. Much is still unknown about the tribochemical reactions that occur during the course of rubbing and the effects this has on the boundary film thickness and friction. Since the rubbing process essentially supplies both mechanical and thermal energy repeatedly over the same surface, the nature of these films must therefore be a dynamic one. Within the limits of their shear and adhesion strength, these films formed will either remain stable or grow in thickness. Outside those limits, they will be gradually desorbed by repeated sliding, or in the worse case, be violently sheared away, accelerating wear. Effective films are formed when the rate of adsorption is greater than or equal to the rate of desorption [2], but these rates may be also dependent on contact conditions, such as temperature, load, speed, material properties, and surface topography. Optimal boundary lubrication design therefore requires knowledge of the conditions suitable for their preferred tribochemical pathways.

Classical boundary lubricants such as fatty acids owe their ability to reduce wear and friction by their ability to adhere and form a tightly packed adsorbed layer on the surface. While it is generally accepted that the adhesion and shear strength of this film increases with the degree of saturation and chain length of the fatty acid, recent studies suggest that the mechanisms at work may be far more complex. Zhang et al. [3] found that the frictional and antiwear behaviour of arachidic acid (C20) were better than that of stearic acid (C18) and behenic acid (C22). Bhuyan et al. [4] observed that, with branched fatty acid methyl ester, shorter chains actually have a lower friction than those of longer chains. When Murakami et al. [5] compared stearic (C18), oleic (C18-1), linoleic (C18-2), and linolenic acids (C18-3) doped in paraffinic oil in a four-ball wear tester, they found that the polyunsaturated fatty acids have higher scuffing loads and showed better frictional stability at high temperatures. In yet another study, Sahoo et al. [6] recently reported that the coefficient of friction (COF) of $n$ hexadecane doped with linoleic acid $(5 \% \mathrm{v} / \mathrm{v})$ decreased over sliding time from 0.09 to roughly 0.04 , while that of stearic acid remained relatively unchanged.

Generally, the linear log-log relationship of film thickness and velocity for elastohydrodynamic lubrication (EHL) theory holds up to the point where film thickness approaches the dimensions of the adsorbed or tribochemically formed boundary layer. However, recent studies have shown that this may not be entirely true for all lubricants. For instance, Spikes [7] observed that with stearic acid in hexadecane solution, film thickness was initially in the order of $2 \mathrm{~nm}$ at low speeds, when the test was conducted at increasing speeds. However, on reducing the speeds, the boundary film thickness was found to remain stable at approximately $10 \mathrm{~nm}$. When the motion was halted, the thick film was lost and the thickness was reduced to $1-2 \mathrm{~nm}$. Similarly with vegetable oils, Biresaw [8] found that at entraining speeds below $0.1 \mathrm{~m} / \mathrm{s}$, and at EHL film thicknesses less than $50 \mathrm{~nm}$, the pressure viscosity coefficient of the oils was comparatively higher. Following the ideas of Luo et al. [9], there may exist not only an immobile adsorbed layer on the surface but the presence of a transition phase above that of the adsorbed layer which is highly viscous. This further raises the question: What defines the boundary of the boundary layer? Is it the static thickness under the test load, or the point where the linearity of elastohydronamic theory is deviated? Clearly, our conventional views of boundary lubrication are insufficient to explain some of the observations made. In this work, the current methods of boundary lubrication study will be briefly reviewed and a new method for the study of boundary films will be proposed, along with pilot test results.

\section{Methods of Boundary Lubrication Study}

The study of boundary films has been traditionally conducted using the pin-on-disk tribometer, reciprocating tribometer and four-ball wear tester. The limitation of these tribometers is that neither the frictional traces nor the wear scar measurements reveal much about the thicknesses of the boundary films generated during the course of rubbing. It is thus difficult to make a logical deduction whether frictional changes, if any, are attributable to changes in the amount of asperity-to-asperity contact, or changes in the viscosity or shear strength of the boundary film itself. To gain a clearer understanding of the nature of these films, it is imperative that friction and boundary film thickness be measured simultaneously in situ.

Studies involving the measurement of lubricant film thickness are not new. Since the invention of the optical interferometry method by Gohar and Cameron [10], it has become an essential tool in tribological and lubricant rheological studies. The method was further developed by a few researchers, most notably by Spikes and coworkers[11, 12]. With the addition of a thin silica layer on the optical disc and the use of computer-assisted image processing, film thicknesses down to $1 \mathrm{~nm}$ can be measured. This enabled the observation and thickness measurement of boundary films. However, as noted by the originators themselves, the study of boundary films using optical method is limited by the fact that the thin chromium layer, responsible for the interferometry effect to work, is easily worn off during sliding [13]. More so is the lesser stated fact that the thickness of a tribochemically formed 
boundary film between metallic surfaces depends on the contact conditions during the running-in process, and the effect of these conditions can only be reproduced in an actual metal-to-metal rubbing experiment. The optical method thus will not suffice since the method inherently requires one of the surfaces to be transparent, and hence, non-metal.

Advances in nanotechnology have brought about the advent of nano-tribological techniques, such as atomic force microscopy (AFM) and surface force microscopy (SFM). With contact pressures in the order of gigapascals achievable by using a tip radius of nanometer dimensions, it attempts to simulate the tribological contact of a single asperity, and can provide both friction and boundary film thickness measurements. However, the method does not replicate the actual shearing conditions in real macro contacts, where flash temperatures at asperity-to-asperity junctions are significantly much higher. In addition, the process of material removal and the presence of entrained wear particles, which may have an influence on the formation of tribochemically reacted films, are not present. As it has been pointed out by Bhusan [14], contact stresses in AFM/LFM studies do not exceed the hardness of the material, which is comparatively much harder at the microscale. As a result, there is usually very little wear. It is worthwhile to mention that in macro-contact rubbing, each asperity will be rubbed the number of times roughly equivalent to the number of asperities across the contact, between intervals in the order of milli or microseconds. If the process of rubbing raises the temperature at these junctions, it follows that each asperity will have very little time to cool before it meets the next asperity. Consequently, flash temperatures would be much higher than those experienced in microscale friction measurements. Although AFM/SFM technique provide an interesting perspective of the shear strength of boundary films at the nano-level, its use as a predictor of their macro-tribological properties is somewhat questionable.

The sliding contact should be viewed upon as a complex dynamic tribological system that evolves over rubbing time. The representative study of the evolution and dynamic growth of these boundary films in practice therefore lies in their thickness measurement in a metallic macro-tribological contact. Studies of film thickness measurement between metallic contacts can be found in the pioneering works dating back to 1958 by Crook [15], and then later in 1965 by Dyson et al. [16], using capacitance methods. However, these studies were directed towards the thickness measurement of EHL rather than boundary films. The method works on the principle that two electrically conductive surfaces separated by a dielectric lubricant film essentially form a capacitor. At the time, measurements were made by manually balancing a capacitance bridge and the task was made more difficult by the frequent short circuiting of the contacting asperities or wear particles being entrained in between the contacting surfaces. It is interesting to note that the optical interferometry method was developed in roughly the same time period. It was naturally favoured over the capacitance method for the measurement of EHL film thickness, since a direct observation of the film thickness profile over the entire contact can be made, whereas the capacitance method can only provide an average value for the film thickness. The advantage, however, is that the sensitivity of the capacitance method increases rapidly as the film thickness approaches nanometer dimensions.

Highly precise (down to femtoFarads) capacitance measurements can now be made easily today with commercially available LCR meters with automatic balancing bridges. However, stable readings of contact impedance would still require short circuiting of the contacts to be avoided. This problem is less when a higher excitation frequency is used. But excessively high frequencies (in the order of $\mathrm{MHz}$ or more) render measurements susceptible to electromagnetic interferences (EMI) in the test environment and would require more specialized shielding.

A new tribometer, named Contact Impedance Tribometer (CIT) has been successfully developed at the Tribology Laboratory of the University of Western Australia. The motivation behind this development was to allow the study of boundary film formation and growth, and their effects on friction and wear in a realistic tribological context. The objective of this paper is to introduce the new method, along with pilot test results using sunflower oil. The study represents the first attempts at monitoring the thickness of boundary films in situ between sliding steel surfaces over long sliding distances.

\section{Apparatus}

The experimental setup is shown in Fig. 1. The setup is basically that of a ball-on-disc tribometer. The main difference is that the ball holder was modified to act as the capacitance probe (see Fig. 2). The rotating disc assembly is belt driven by an AC servomotor, giving a range of 1$1000 \mathrm{rpm}$ at the disc. The capacitance and resistance measurements were made simultaneously using a GWInstek LCR 821 meter. COF was measured using strain gauges bonded onto the load cantilever arm. The sensitivity of the friction force measurement is about $0.632 \mathrm{~N} / \mathrm{V}$, and provides frictional measurement with an accuracy of 0.002 at $1 \mathrm{~kg}$ load. Wear was continuously monitored by measurement of the wear track width via a Dino-lite AM413ZT digital microscope fitted above the disc. All measurements were recorded simultaneously by a computer. 


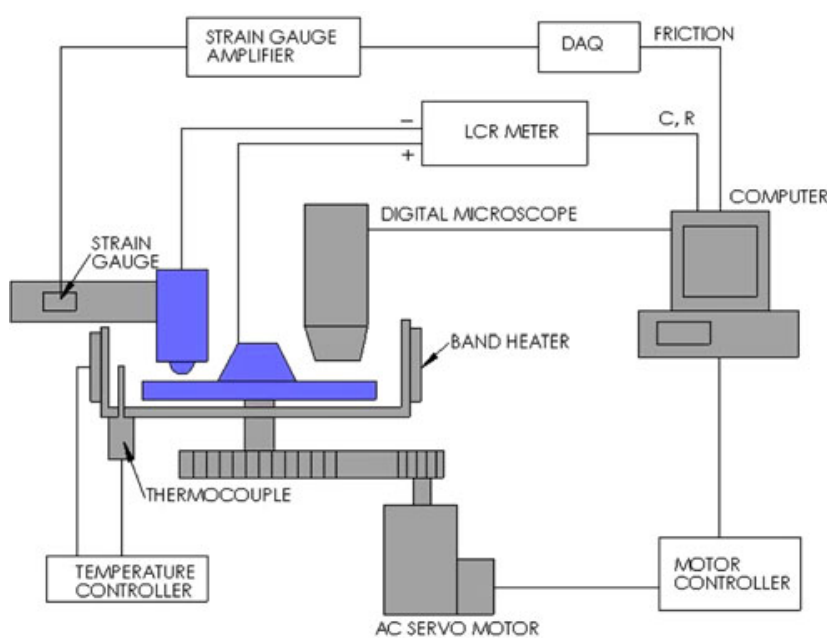

Fig. 1 Schematic illustration of the experimental setup

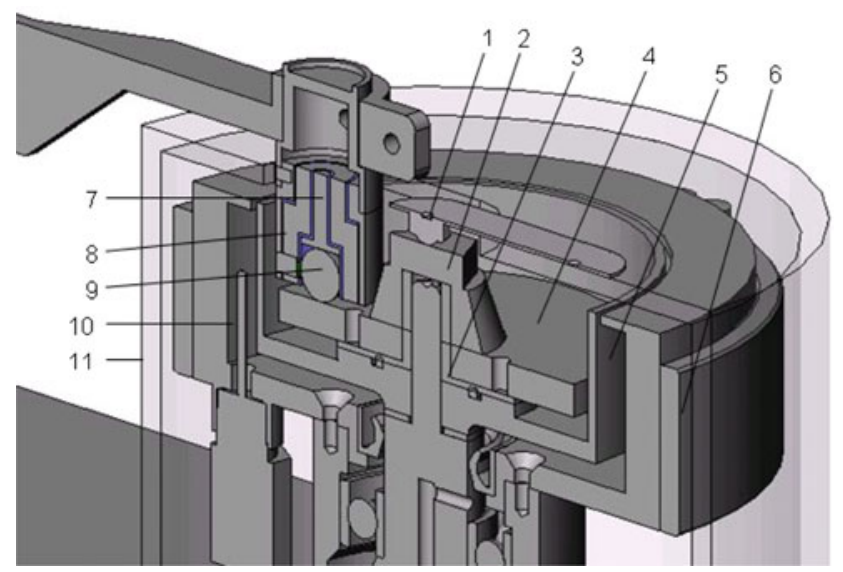

Fig. 2 Sectional 3D view of test cell assembly. 1 Brass contact with cantilever spring, 2 securing nut; 3 electrical insulating sleeve, 4 test disc, 5 heating oil chamber, 6 band heater, 7 negative electrode, 8 guard ring, $98 \mathrm{~mm}$ diameter bearing ball, 10 thermocouple, 11 cover

The $8 \mathrm{~mm}$ diameter bearing ball (9) was secured via a nylon tipped grub screw, onto the negative electrode seat (7) of the capacitance probe. The bearing ball thus served as both the slider and the negative electrode for impedance measurements. The capacitance probe comprises of a guard ring (8) surrounding the bearing ball to eliminate fringing capacitance, thereby improving the accuracy of the measurement. The guard is in turn enclosed in an outer shield to avoid ground loops due to small differences in electrical ground between the rig and the meter. The counter surface is a flat $60 \mathrm{~mm}$ diameter disc (4), and served as the positive electrode. It was electrically connected by the securing nut and brass contact (1), which were kept in constant contact using an aluminium cantilever spring. The electrode assembly, the securing nut and the wear disc had been electrically insulated from the rest of the setup. The bearing ball and the flat disc were connected to the LCR meter via $1 / 2 \mathrm{~m}$ long, RG174/U shielded coaxial cables. The excitation current used for the impedance measurement was $200 \mathrm{kHz}$ at $0.25 \mathrm{~V}$, which corresponds to the manufacturer's rated accuracy of approximately $1 \%$ of reading.

Temperature control was achieved by the use of a band heater (6) clamped around the assembly housing and a thermocouple (10) mounted in the heating chamber (5), where silicone oil serve as the heating oil to provide uniform heating. The lubricant cell was sealed from the heating chamber by an ' $\mathrm{O}$ ' ring, to prevent cross contamination of the test lubricant with the heating oil. This setup allows the cell to be removed easily for cleaning after the test.

\section{Measurement of the Dielectric Constant and Prediction at High Pressures}

To convert capacitance data to film thickness, the dielectric constant of the lubricant at ambient and contact pressure must be known. This can be measured using the same capacitance probe shown in Fig. 2. However, the guard ring of the capacitance probe was re-wired as the negative electrode, and the bearing ball was replaced with a spherical electrical insulator, such that there was a parallel gap of approximately $1 \mathrm{~mm}$ between the flat surface of the guard ring and the top side of the test disc. First, the capacitance of the air gap, $C_{\text {air }}$ was measured, where $\varepsilon_{\text {air }}$ is taken as 1.00059 . The test lubricant was then slowly poured into the cell until the gap was fully flooded and the capacitance of the test lubricant is then measured. Measurements were made at the test excitation frequency of $200 \mathrm{kHz}$ and $0.25 \mathrm{~V}$, in the parallel mode. Capacitance readings were recorded continuously throughout this entire procedure until thermal equilibrium was reached and there was little change in the capacitance readings. The dielectric constant of the bulk lubricant at the test temperature was then calculated as follows:

$\varepsilon_{\mathrm{b}}=\frac{C_{\mathrm{b}}}{C_{\mathrm{air}}} \varepsilon_{\mathrm{air}}$

The dielectric constant of the boundary layer, however, must be determined at the average Hertzian contact pressure and test temperature. This can be done experimentally with the use of a high pressure condenser, or by employing empirical methods. Details of the empirical method used in this work are described in the Appendix.

\section{Calculation Method for the Determination of the Boundary Film Thickness}

The film thickness calculation method used is similar to that proposed by Dyson et al. [16], which requires 


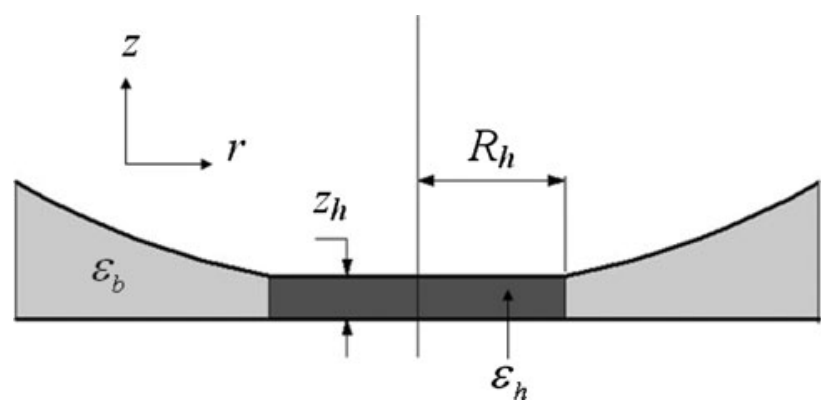

Fig. 3 Capacitance-film thickness model based on Dyson et al.'s [17] method, where $z_{\mathrm{h}}=$ film thickness, $R_{\mathrm{h}}=$ radius of the Hertzian contact area, $\varepsilon_{\mathrm{b}}=$ bulk fluid dielectric constant, and $\varepsilon_{\mathrm{h}}=$ the dielectric constant of the fluid at Hertzian contact pressure

assumption of the shape and area of the contact zone. The capacitance of the ball can be evaluated in two parts, i.e., the capacitance at the Hertzian contact and the capacitance outside the contact. Dyson et al. [16] assumed that the Hertzian contact is a perfectly smooth and flat area in the study of EHL film thickness (see Fig. 3). For boundary lubrication, however, this assumption cannot be used, because the area of contact does not remain constant during sliding, and the surfaces will not be perfectly smooth. While most boundary lubricants reduce the wear rate, they cannot prevent wear completely during the course of sliding. Some lubricant films may be sheared away, only to reform at a later time, during which wear may take place in between; while others may require a longer period of running-in before a sustainable film is formed. In either case, the assumption of the Hertzian area of contact based on the original geometry of the surfaces will no longer be valid. This means that the wear scar diameter during the course of sliding must be known for the calculation of the film thickness to be accurate.

\subsection{Capacitance Contribution Within the Apparent Area of Contact}

According to Johnson [17], for two randomly rough surfaces in contact, the ratio of the real area of contact to the apparent area of contact is directly proportional to the mean apparent contact pressure, i.e.,:

$\frac{A_{\mathrm{r}}}{A_{\mathrm{a}}}=\pi^{1 / 2}\left(\sigma \kappa_{\mathrm{s}}\right)^{-1 / 2}\left(\frac{p_{\mathrm{a}}}{E^{\prime}}\right)$

where $A_{\mathrm{r}}$ is the real area of contact, $A_{\mathrm{a}}$ is the apparent area of contact, $\sigma$ is the surface roughness standard deviation, $\kappa_{\mathrm{s}}$ is the mean curvature of the surface profile, $p_{a}$ is the average apparent contact pressure, and $E^{\prime}$ is the reduced Young's modulus of elasticity. It can be seen from Eq. 1 that for a constant surface roughness, the real area of contact is directly proportional to the load, i.e., $A_{\mathrm{r}} \propto p_{\mathrm{a}} A_{\mathrm{a}}$.
It follows that, for a constant load, if the apparent area of contact increases due to wear, the mean apparent contact pressure must decrease accordingly, so that $A_{\mathrm{r}}$ remains roughly the same during the sliding process. As long as the surface roughness is not significantly altered during sliding, this assumption will hold true. For this reason, the surfaces used in this work have been highly polished, so that running-in wear and the change in surface roughness during rubbing are not significant.

As schematically illustrated in Fig. 4a, the asperities are deformed at the real areas of contact. In the presence of a boundary film, the opposing asperities would be separated by a distance equal to the thickness of the boundary film, $z_{\mathrm{r}}$. The other areas not in contact (within the apparent area), $A_{\mathrm{nr}}=A_{\mathrm{a}}-A_{\mathrm{r}}$, can be modeled as being separated by a composite film of two boundary layers and the entrapped bulk fluid. For simplicity, we assume that the average separation is equivalent to the sum of the boundary film thickness, $z_{\mathrm{r}}$, and the combined surface roughness, $R_{\mathrm{a}}$, of the two surfaces, as shown in Fig. 4b. Sellgren et al. [18] have shown that for smooth surfaces and high loads, the ratio $A_{\mathrm{r}} / A_{\mathrm{a}}$ approaches unity, so $A_{\mathrm{r}}$ can be calculated from Hertzian contact equations. Since mirror polished surfaces and moderate Hertzian pressures of 0.69 GPa were used in this study, it can be assumed that $A_{\mathrm{r}} / A_{\mathrm{a}}=1$ at the beginning of sliding, and the capacitive contribution of the boundary film at the real areas of contact is:

$C_{\mathrm{r}}=\frac{\varepsilon_{\mathrm{r}} \varepsilon_{\mathrm{o}} A_{\mathrm{r}}}{z_{\mathrm{r}}}$

where $A_{\mathrm{r}}$ is the Hertzian contact area, $\varepsilon_{\mathrm{r}}$ is the dielectric constant of the boundary layer at the average Hertzian pressure and test temperature, $\varepsilon_{\mathrm{O}}$ is the permittivity of free space, and $z_{\mathrm{r}}$ is the thickness of the boundary film.The

(a)

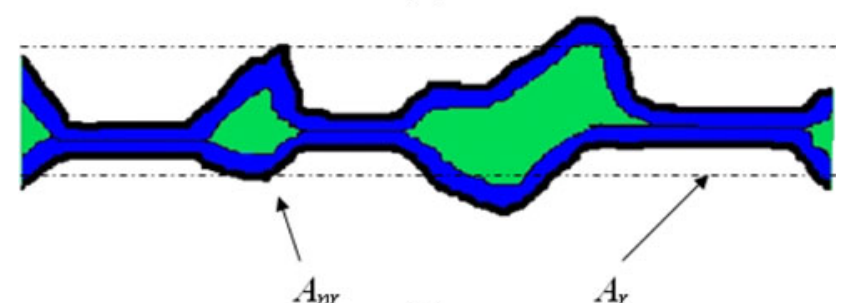

(b)

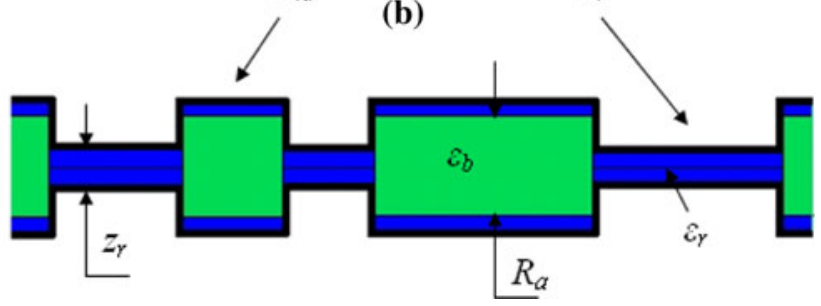

Fig. 4 a Realistic contact between sliding surfaces, b Modified capacitance-film thickness model at the apparent area of contact 
capacitance contribution of the composite film at the other areas within the apparent area of contact will be:

$C_{\mathrm{nr}}=\frac{\varepsilon_{\mathrm{r}} \varepsilon_{\mathrm{b}} \varepsilon_{\mathrm{o}} A_{\mathrm{nr}}}{\varepsilon_{\mathrm{b}} z_{\mathrm{r}}+\varepsilon_{\mathrm{r}} R_{\mathrm{a}}}$

where $\varepsilon_{\mathrm{b}}$ is the dielectric constant of the bulk fluid at atmospheric pressure, $A_{\mathrm{nr}}$ is the non-contact areas within the apparent area of contact, and $R_{\mathrm{a}}$ is the combined surface roughness. To calculate $A_{\mathrm{nr}}$, the apparent area of contact, $A_{\mathrm{a}}$ at the time of rubbing must be known. This can be done by monitoring the growth of the wear track width during the test. The wear track width provides an estimate of the ball wear scar diameter and hence $A_{\mathrm{a}}$, where $A_{\mathrm{nr}}=A_{\mathrm{a}}-A_{\mathrm{r}}$.

It can be seen from Eq. 3 that if the boundary film thickness, $z_{\mathrm{r}}$ is comparatively larger than the surface roughness, $R_{\mathrm{a}}\left(z_{\mathrm{r}} \gg R_{\mathrm{a}}\right)$, Eq. 3 reduces to $C_{\mathrm{nr}}=\varepsilon_{\mathrm{o}} \varepsilon_{\mathrm{r}} A_{\mathrm{nr}} / z_{\mathrm{r}}$. Practically, there will be little wear for thick boundary films, so the capacitive contribution of $C_{\mathrm{nr}}$ would be negligible since $A_{\mathrm{nr}}$ is small. On the other hand, if $z_{\mathrm{r}} \ll R_{\mathrm{a}}$, Eq. 3 reduces to $C_{\mathrm{nr}}=\varepsilon_{\mathrm{o}} \varepsilon_{\mathrm{b}} A_{\mathrm{nr}} / R_{\mathrm{a}}$. In this case, since the load will be mainly supported by the asperities at the real areas of contact, the local pressures at the non-contact areas must be close to the ambient pressure. For thin films, where the impact of wear cannot be ignored, $A_{\mathrm{nr}}$ could be much greater than $A_{\mathrm{r}}$, but since $R_{\mathrm{a}}$ is large compared to $z_{\mathrm{r}}$, the total capacitance will, in effect, still be dominated by $C_{\mathrm{r}}$.

\subsection{Capacitance Contribution Outside the Apparent Area of Contact}

Outside the apparent area of contact, the capacitance is modeled by a half sphere (see Fig. 5). The capacitive contribution outside the Hertzian contact, $C_{\mathrm{b}}$ is thus calculated by summing the capacitive contribution of each infinitesimal area of the probe, $2 \pi r \cdot \mathrm{d} r$. For a non-deformed bearing ball (with radius $R_{\mathrm{b}}$ ) in contact with the flat disc is, the vertical coordinate, $z$, at any point along the radius, $r$, of the ball is:

$z=R_{b}-\sqrt{R_{b}^{2}-r^{2}}$

During the course of sliding, this vertical distance will be reduced by the wear depth of the ball, $z_{w}$ and raised by the boundary film thickness, $z_{\mathrm{r}}$, as shown in Fig. 5. Hence,

$C_{\mathrm{b}}=\int_{R_{\mathrm{w}}}^{R_{\mathrm{b}}} \frac{2 \pi r \cdot \varepsilon_{\mathrm{b}} \varepsilon_{\mathrm{o}}}{\left(R_{\mathrm{b}}-\sqrt{R_{\mathrm{b}}^{2}-r^{2}}\right)-z_{\mathrm{W}}+z_{\mathrm{r}}} \mathrm{d} r$

where $z_{\mathrm{w}}$ is the wear depth of the ball and $R_{\mathrm{w}}$ is the radius of the wear scar. At sliding time, $t=0, z_{\mathrm{w}}=z_{\mathrm{d}}$, and $R_{\mathrm{w}}=R_{\mathrm{h}}$, where $z_{\mathrm{d}}$ is the Hertzian maximum deflection of

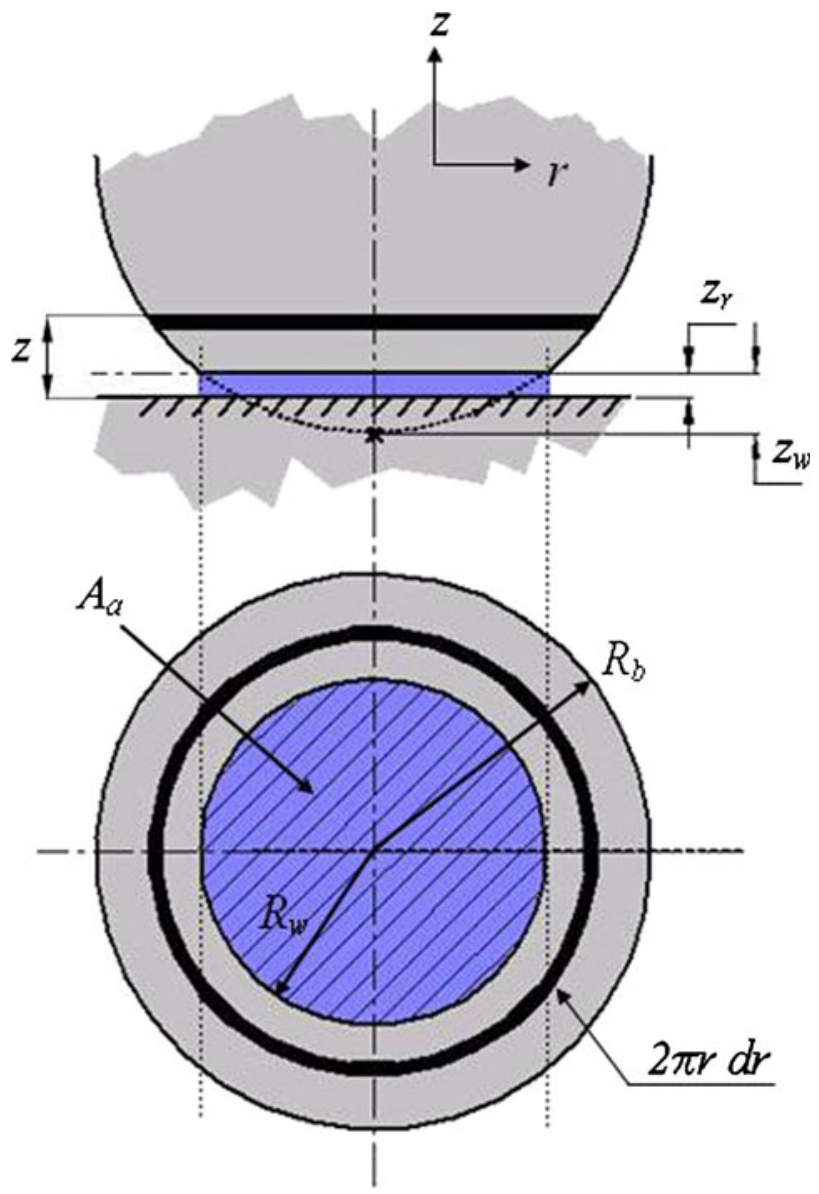

Fig. 5 Schematic illustration of the capacitance contribution outside the apparent area of contact. Note that the apparent area of contact has been exaggerated for clearer illustration

the ball under the test load and $R_{\mathrm{h}}$ is the radius of the Hertzian contact area.

\subsection{Conversion of Capacitance Values to Film Thickness Values}

The total capacitance, $C_{\mathrm{t}}$ is simply the sum of the capacitances derived above:

$C_{\mathrm{t}}=C_{\mathrm{r}}+C_{\mathrm{nr}}+C_{\mathrm{b}}$

Once the dielectric constants of the test lubricant at atmospheric and contact pressure have been determined, the predicted capacitances can then be calculated for film thicknesses varying from $0.1 \mathrm{~nm}$ to $0.1 \mu \mathrm{m}$, and for wear scar diameters varying from the initial Hertzian contact radius of $56.3 \mu \mathrm{m}$ (current work) to $250 \mu \mathrm{m}$. This was done using MATLAB. An example of the capacitance-film thickness relationship for sunflower oil is shown in Fig. 6, using an iterative step size of $1 \mu \mathrm{m}$ and $1 \mathrm{~nm}$ for the wear scar radius and the film thickness, respectively. After the test, the recorded capacitance values were then converted 


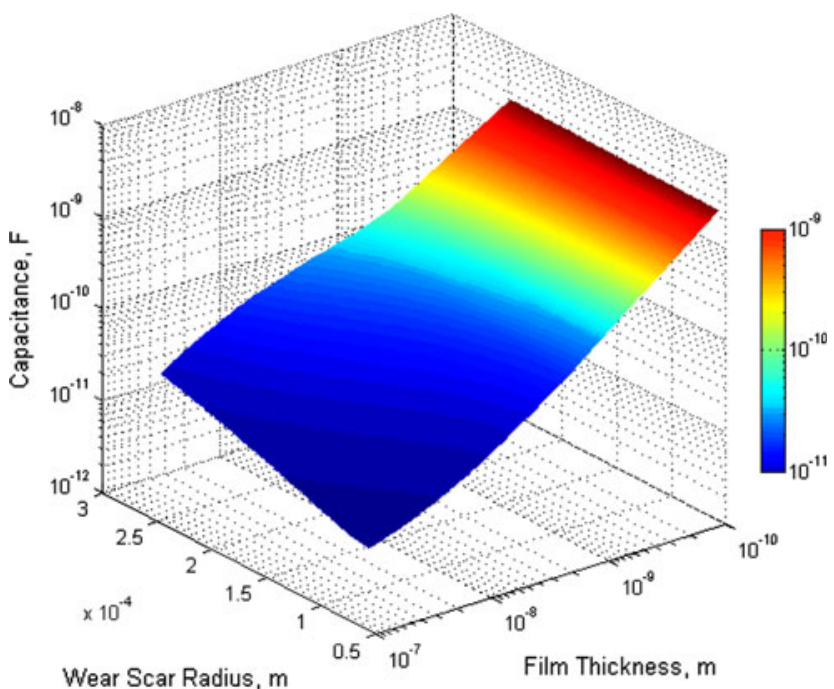

Fig. 6 Capacitance-film thickness-wear scar radius relationship for sunflower oil

to film thickness values by interpolation of the 3D relationship using MATLAB given time associated inputs of the wear track width.

\section{Experimental Details}

The $8 \mathrm{~mm}$ diameter bearing ball used is a commercially available bearing ball with a surface roughness $R_{\mathrm{a}}$ of $11 \mathrm{~nm}$ and hardness of 63-65HRB. The $60 \mathrm{~mm}$ diameter wear disc is made of 52100 steel, purchased from Bohler. After machining, each disc was heat-treated to $860{ }^{\circ} \mathrm{C}$ for $20 \mathrm{~min}$, quenched in vegetable oil and then tempered at $150{ }^{\circ} \mathrm{C}$ for another $20 \mathrm{~min}$, yielding the final hardness of $810 \pm 10 \mathrm{HV}$. The modulus of elasticity of both the disc and ball are taken as $210 \mathrm{GPa}$. Before each experiment, the wear disc was ground with $\mathrm{SiC}$ paper of grit 120, 240, 500, and 1200 , and then finally polished to a mirror finish of $13 \mathrm{~nm} R_{\mathrm{a}}$ with diamond suspension at 6,3 , and $1 \mu \mathrm{m}$. The disc was then washed once with cold tap water and ethanol after polishing, and wiped dry with soft tissue paper. Prior to installation, both the disc and a new ball were ultrasonically bathed in acetone for $15 \mathrm{~min}$, briefly washed again with ethanol, hexane, and acetone, and finally blown dry with hot air. The ball was then secured in the capacitance probe by a nylon tipped grub screw, and the resistance was checked with a handheld multimeter to ensure good connection with the electrode seat.

After both the disc and bearing ball had been securely mounted, the LCR meter was then compensated to offset the parasitic capacitance and impedance of the leads during measurement. This was done by an 'open' and 'short' circuit test, as required by the LCR meter's zeroing procedure. During the 'short' circuit test, the capacitance probe was loaded with the same load used in the test. This ensured that the resistance was set to zero at the initial Hertzian contact.

The test lubricant was then slowly poured onto the wear disc and allowed to fully cover the latter, until the gap between the electrode guard and the disc was entirely flooded in the test lubricant. The whole cell, including the test lubricant was then gradually brought to the test temperature $\left(40{ }^{\circ} \mathrm{C}\right.$ in these experiments), and then allowed to sit for $30 \mathrm{~min}$ to ensure that thermal equilibrium was reached. Finally, the bearing ball was then loaded with the dead weight, giving a test load of $0.7 \mathrm{~kg}$ at the contact. The mean Hertzian contact pressure and contact radius at this test load was $0.69 \mathrm{GPa}$ and $56.3 \mu \mathrm{m}$, respectively.

For this work, the rotational speed was set at $10 \mathrm{rpm}$, corresponding to a sliding speed of $0.02 \mathrm{~m} / \mathrm{s}$. The theoretical central film thickness based on EHL theory would be less than $5 \mathrm{~nm}$ at this test temperature. This is less than the combined surface roughness of $17 \mathrm{~nm}$, so the lubrication regime here would be predominantly boundary lubrication. The measurement speed of the LCR meter had been set at the "slow" setting of $0.896 \mathrm{~s}$, and the averages of 7 measurements were continuously recorded. Friction signals were sampled at 1000 samples per second and then averaged over the time taken for one wear disc revolution to complete. The digital microscope was set to capture a video of the wear track at 30-min frame intervals for the duration of the experiment.

After the test, both the ball and disc were removed, washed with ethanol and hexane, and then wiped dry with a soft tissue. The final wear scar diameter and wear track width were then measured using an optical microscope. Wear progression during the course of sliding was then measured from the captured video frames, by scaling the pixels according to the final wear track width. The magnification of the digital microscope was set at $36 \times$ and the precision of wear measurement at this magnification is about $10 \mu \mathrm{m}$ per pixel.

\section{Test Lubricant}

Sunflower oil was selected for the pilot test and its properties at $40{ }^{\circ} \mathrm{C}$ and ambient pressure, unless otherwise stated, are as follows: density, $\rho-0.901 \mathrm{~g} / \mathrm{cm}^{3}$; refractive index at $23{ }^{\circ} \mathrm{C}$, $n-1.476$; dynamic viscosity, $\eta-30.3 \mathrm{mPas}$; dielectric constant, $\varepsilon_{\mathrm{b}}-2.575$; dielectric constant at $0.69 \mathrm{GPa}, \varepsilon_{\mathrm{r}}-2.647$. The refractive index was measured under ambient conditions using an Abbe Refractometer and the dynamic viscosity at $40{ }^{\circ} \mathrm{C}$ was determined using the Brooksfield Cone/Plate Digital Viscometer. The dielectric constant under ambient pressure was measured by the method as described in Sect. 4, 
and the constant at $0.69 \mathrm{GPa}$ was predicted using the Onsager equation and the modified Tait-Tammann equation (refer to Appendix for calculation method). The sunflower oil used is a refined, bleached, and deodorized (RBD) vegetable oil, obtained from Cargill. Measurements using Gas Chromatography Mass Spectrometer (GCMS) revealed that the sunflower oil has a high level of polyunsaturated fatty acid content, with the major constituents as follows: palmitic acid $9.5 \%$, stearic acid $4.6 \%$, oleic acid $30 \%$, linoleic acid $46.1 \%$.

\section{Experimental Results}

Contact between asperities or the entrainment of large metallic wear particles in the contact zone usually results in a significant decrease in resistance and a negative capacitance reading on the LCR meter. This usually occurs for very thin films. In smoothing the signal, the negative capacitance readings were changed to infinity (mathematically corresponding to a zero film thickness). Ten consecutively recorded readings were then averaged, and the infinity values were rejected. This effectively retained capacitance readings that were measurable for at least a minute.

Figure 7 shows the smoothed capacitance and resistance traces acquired simultaneously by the LCR meter. During the test, capacitance values reached as low as $36 \mathrm{pF}$ and resistance reached as high as $150 \mathrm{k} \Omega$. The friction and film thickness plots of sunflower oil are shown in Fig. 8. The most notable observation is the appreciable growth in the film thickness, which is mirrored by a decrease in friction. Initially, the boundary film thickness varied between 3 and $5 \mathrm{~nm}$. After approximately $1 \mathrm{~h}$, there seemed to be a

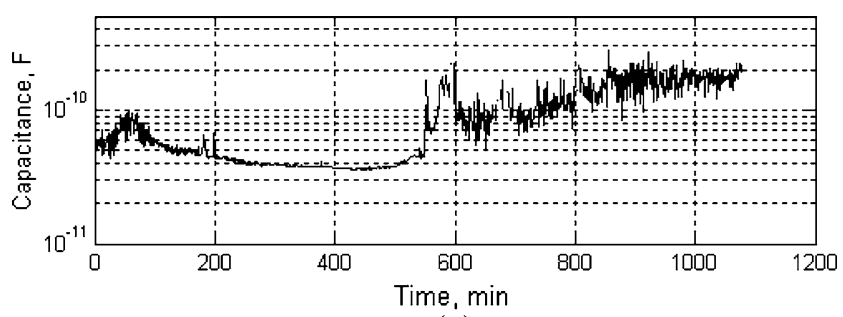

(a)

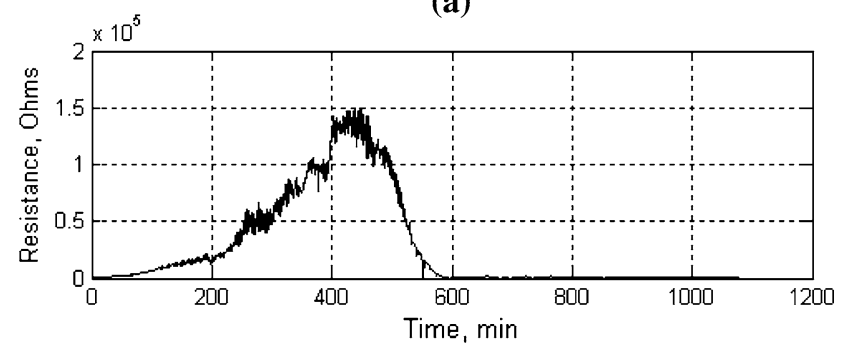

(b)

Fig. 7 a Capacitance trace versus sliding time, b resistance trace versus sliding time

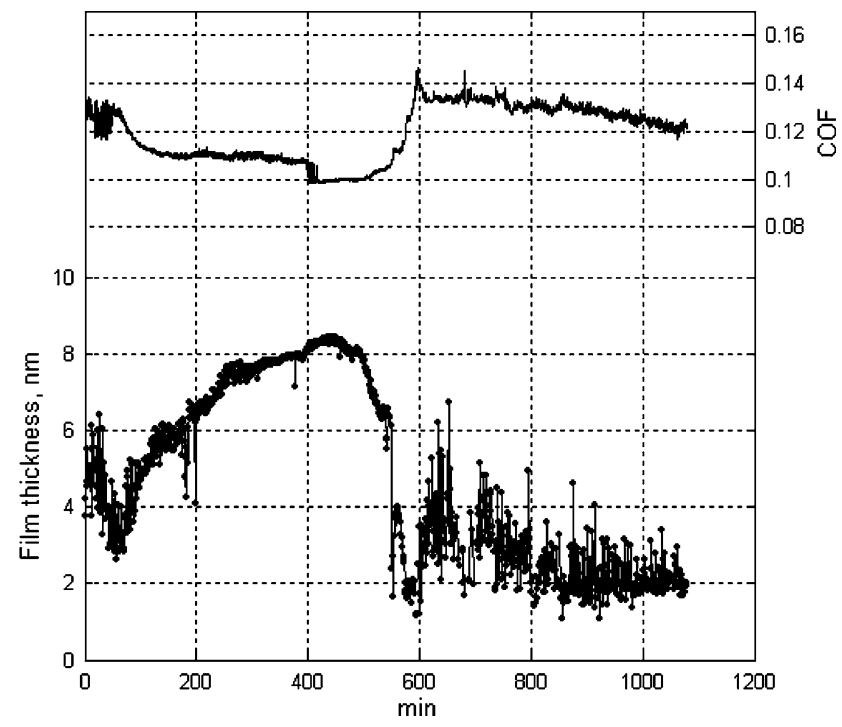

Fig. 8 Film thickness and COF versus sliding time plot

transition, where the film thickness appeared to steadily increase over time. The frictional trace was also observed to be much smoother after this transition. It is uncertain whether this is due to a completion of the running-in phase or a tribochemical reaction. The film thickness continued to increase until a maximum of about $8 \mathrm{~nm}$ at approximately $450 \mathrm{~min}$, after which it appeared to decline rapidly. After about $560 \mathrm{~min}$, the film seemed to have collapsed almost completely, though it is uncertain at this point why this occurred.

Repeat tests showed some variation in the measured peak film thickness from 7 to $12 \mathrm{~nm}$ and in the film breakdown time from 300 to $600 \mathrm{~min}$, presumably due to high sensitivity to test conditions. However, a distinct trend was observed in all the friction and film thickness traces: a short running-in period, followed by a gradual growth in film thickness accompanied by a decrease in friction, and finally a rapid decline in the film thickness, accompanied by a return to high frictional values and a high wear rate.

Wear track width measurements show that the sunflower oil was effective in preventing wear when the film was present and growing in thickness (see Fig. 9). Wear increased rapidly once this film had collapsed. The initial wear track width is in close agreement with the theoretical Hertzian contact diameter. Examination of the final ball wear scar and wear track (see Fig. 10) under the optical microscope revealed a reddish brown topography that resembled that of corrosion products on the wear track, while no sign of this coloration was observed on the ball wear scar. Further studies would be required to determine the chemical nature of this substance.

It is postulated that the observed film growth was due to the polymerization of fatty acid chains. The concept of the 


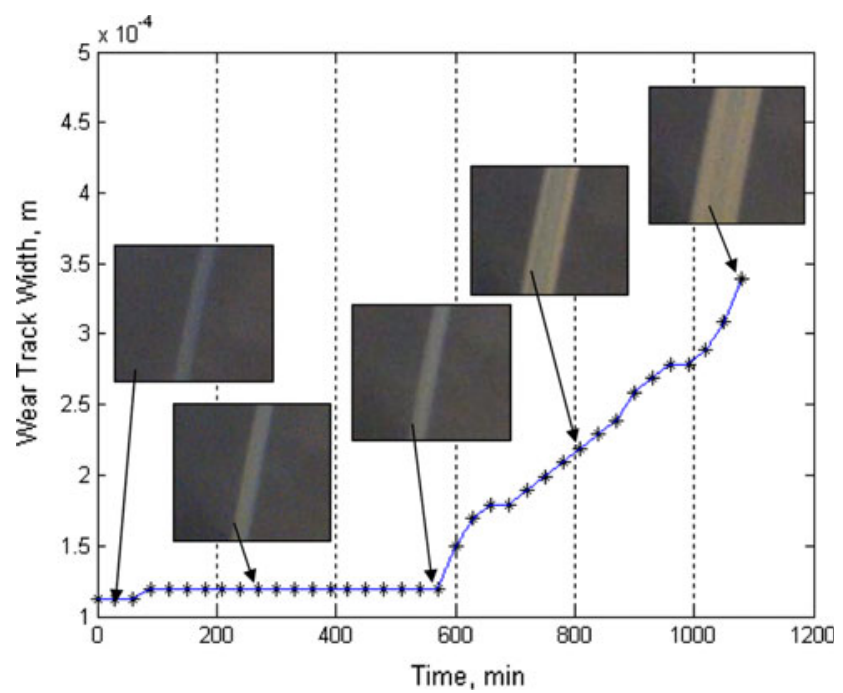

Fig. 9 Wear track widths versus sliding time measured using images captured at 30-min intervals from a digital microscope
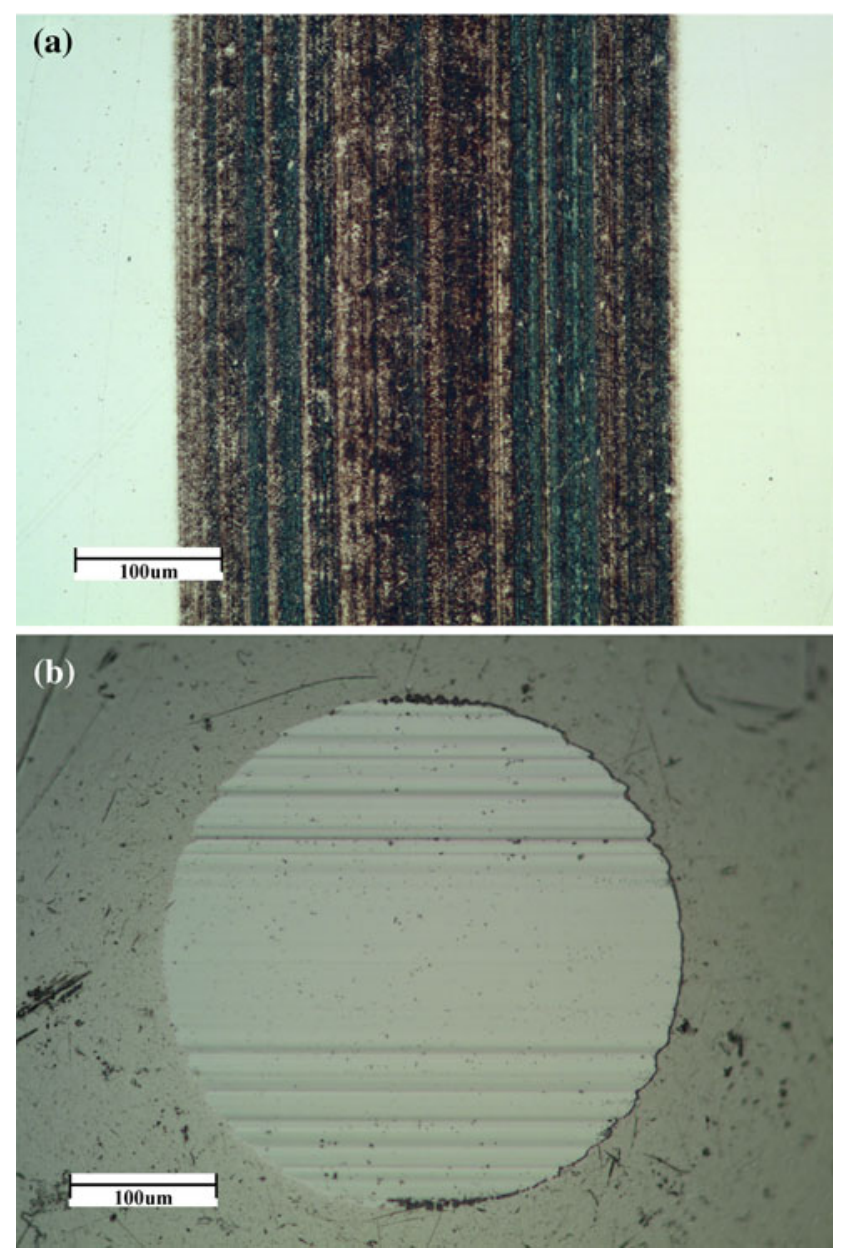

Fig. 10 Optical micrographs of $\mathbf{a}$ wear track on the disc, and $\mathbf{b}$ ball wear scar formation of polymeric films reducing friction and wear is not new. It was first introduced by Furey [19] involving the use of a $\mathrm{C}_{36}$ dimer acid/ethylene glycol blend. Sunflower oil contains a large number of double bonds, $\mathrm{C}=\mathrm{C}$ due to the high proportion of polyunsaturated fatty acids in its triglyceride structure. The double bonds are largely responsible for the oil's poor oxidative stability, but are also essential to the polymerization process. The polymerization process occurs by the reaction of the carboxyl group of one fatty acid with the double bond of another. Both high temperatures and the presence of iron catalysts (which would always be present to some extent in the form of microwear particles) have been known to favour this process [20].

\section{Discussion on the Accuracy and Limitations of the Method}

One major concern regarding the accuracy of film thickness measurement via capacitance methods has been the uncertainty of the dielectric constant. The dielectric constant is not only influenced by pressure and temperature of the contact, but is also sensitive to the presence of water. While it is possible to make reasonable assumptions on what the local pressures and average contact temperature may be, the greatest uncertainty arises from the fact that, during sliding, a change in the molecular structure of the boundary film due to tribochemical reactions would likely affect its dielectric constant. An increasing dielectric constant due to oxidation or polymerization will, in effect, shift the capacitance versus film thickness curve up, as shown in Fig. 11. The dielectric constant of a vegetable oil blend of cottonseed and sunflower oil was reported to have increased by as much as 0.5 during the accelerated oxidation process [21]. It should be noted, however, that the impact of a change in the dielectric constant on the accuracy decreases for a decreasing film thickness. For instance, an increase of the dielectric constant of sunflower oil from 2.65 to 3.15 would underestimate the actual film thickness of $73 \mathrm{~nm}$ by $10 \mathrm{~nm}$; while an actual film thickness of $3.3 \mathrm{~nm}$ would be underestimated by only $0.5 \mathrm{~nm}$ (see Fig. 11).

Another limitation of the method is that an assumption on the species adsorbed on the surface must be made for the dielectric constant to be determined. This does not pose a problem for pure oils, such as refined sunflower oil, since the adsorbed boundary film is of the same chemical makeup as the bulk fluid. However, in the case of base oils blended or doped with one or more additives, it must be assumed that the dielectric constant of the boundary film will be that of the more polar constituent. The same limitation also applies for the optical interferometry method, since the refractive index of the film must be known in order to calculate the film thickness. 


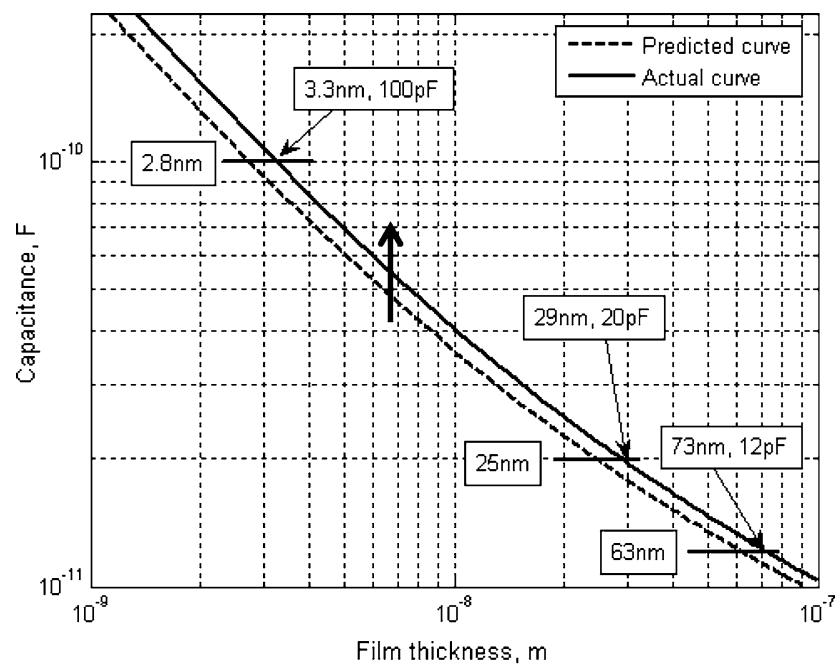

Fig. 11 Chart showing the actual film thickness curve versus the predicted film thickness curve for an increase in the dielectric constant of the boundary film from 2.65 to 3.15

In the present model, it is assumed that, as the apparent area of contact increases due to wear, the real area of contact remains the same during sliding. If this assumption is incorrect, and the real area of contact grows significantly during sliding, then the measurement method will produce erroneous results. Conservatively, the accuracy of the method is maintained as long as wear is not significantly excessive, as in the present case with sunflower oil, when a relatively thick film is present.

There is also a concern that the generation of capacitance, would polarize the boundary film formed, or electrochemically interfere with the tribochemical process. For this reason, a low electrical potential of $0.25 \mathrm{~V}$ has been chosen for the impedance measurement. Lower potentials can be selected but this reduces the accuracy of the LCR meter. To address this concern, the test was repeated under the same conditions without connecting the capacitance sensor to the LCR meter, and results revealed the same coloration on the wear track, together with similar friction and wear trace characteristics, indicating that the electrical potential chosen for this work had a negligible effect on the tribochemical process.

While the capacitance method inherently possesses some flaws and limitations, it is the most direct method to measure the boundary film thickness between sliding metallic surfaces over long periods of time, and to monitor its changes with respect to wear and friction-a task otherwise not possible with the optical interferometry method.

\section{Conclusion}

A new method for the simultaneous measurement of friction, wear, and boundary film thickness has been developed. The motivation behind this development was to allow for the studies of boundary film growth under practical tribological conditions. Boundary films formed by sunflower oil have been shown to grow to thicknesses greater than the expected heights of their adsorbed monolayers. It is postulated that this growth was due to the polymerization of unsaturated fatty acid chains. It would thus be of interest to compare the results obtained herein for sunflower oil, with other plant-derived oils with different degrees of unsaturation.

Acknowledgments We thank the School of Mechanical Engineering, University of Western Australia, for its support in the preparation of this paper, in particular, Mike Reid, from the School's workshop for the manufacture of the test rig. We also thank Cargill for their generosity in supplying the oils.

\section{Appendix}

The dielectric constants for the fluids tested in Dyson et al.'s [16] work was measured by Galvin et al. [22] The latter compared experimental values with theoretical prediction using the well-known Clausius-Mosotti (CM) equation:

$\frac{\varepsilon-1}{\varepsilon+2}=\rho \cdot Y_{\mathrm{o}}$

where $Y_{\mathrm{o}}$ is a constant. They found that the CM equation is relatively accurate for non-polar fluids (such as mineral oils). With polar liquids, however, such as fatty acids and ester lubricants, the method considerably overestimates the dielectric constant, particular at high pressures. The general consensus on this discrepancy is that the $\mathrm{CM}$ equation does not take into account the fact that the polarizability of a polar molecule does not vary linearly with density. A more appropriate formula for polar fluids was derived by Onsager [23]:

$\frac{\left(\varepsilon-\varepsilon_{\infty}\right)\left(2 \varepsilon+\varepsilon_{\infty}\right)}{\varepsilon\left(\varepsilon_{\infty}+2\right)^{2}}=\rho \cdot Y_{1}$

where $Y_{1}$ is a constant and $\varepsilon_{\infty}$ is the dielectric constant at high frequencies, which is usually approximated using the Maxwell relation $\varepsilon_{\infty}=n^{2}$, where $n$ is the refractive index. Good correlation between experimental data and calculation was found for moderately polar liquids $(\varepsilon<5)$ by Valisko et al. [24] using the Onsager equation. Since most boundary lubricants would either be a pure polar liquid or a blend of polar components in a non-polar liquid, the Onsager equation would be more suitably applied.

Both Eqs. 7 and 8 require the density at the Hertzian contact pressure to be known before the dielectric constant can be calculated. A convenient formula commonly used in the field of EHL relating density to pressure is the 
formula by Dowson and Higginson [25]: $\rho / \rho_{\mathrm{o}}=1+$ $[0.6 p /(1+1.7 \mathrm{p})]$. However, it has been shown by several workers that this equation tends to overestimate the density, particularly at high pressures [26]. The equation of state generally regarded as the most accurate, comes in the form of the modified Tait-Tammann equation [27]:

$\rho=\frac{\rho_{o}}{1-C \ln \left(\frac{B+p}{B+p_{\mathrm{o}}}\right)}$

where $B$ and $C$ are constants, $p_{\mathrm{o}}$ is the ambient pressure, $p$ is the pressure of interest, and $\rho_{\mathrm{o}}$ is the density of the fluid at ambient pressure. Bair [28] has also recommended the use of this formula for the quantitative study of EHL contacts. The $B$ and $C$ constants for 13 natural fats and oils, and two pure triglycerides (triolein and trilarin) were evaluated by Acosta et al. [29] at 30, 40, 60, and $80{ }^{\circ} \mathrm{C}$, and the constants $B=110$ and $C=0.07325$ has been chosen for this current work.

\section{References}

1. Bowden, F.P., Tabor, D.: The Friction and Lubrication of Solids. Clarendon Press, Oxford (1950)

2. Hsu, S.M.: Boundary lubrication: current understanding. Tribol. Lett. 3, 1-11 (1997)

3. Zhang, P.Y., Xue, Q.J., Du, Z.L., Zhang, Z.J.: The tribological behaviours of ordered system ultrathin films. Wear 254, 959-964 (2003)

4. Bhuyan, S., Sundararajan, S., Yao, L., Hammond, E.G., Wang, T.: Boundary lubrication properties of lipid-based compounds evaluated using microtribological methods. Tribol. Int. 22(2), 167-172 (2006)

5. Murakami, T., Sakamoto, H.: Lubricating properties of vegetable oils and paraffinic oils with unsaturated fatty acids under highcontact pressure conditions in four-ball tests. J. Synth. Lub. 20(3), 183-277 (2003)

6. Sahoo, R.R., Biswas, S.K.: Frictional response of fatty acids on steel. J. Colloid Interface Sci. 333(2), 707-718 (2009)

7. Spikes, H.A.: Direct observation of boundary layers. Langmuir 12, 4567-4573 (1996)

8. Biresaw, G.: Elastohydrodynamic properties of seed oils. J. Am. Oil Chem. Soc. 83(6), 559-566 (2006)

9. Luo, J.B., Wen, S.Z., Huang, P.: Thin film lubrication. Part I: study on the transition between EHL and thin film lubrication using a relative optical interference intensity technique. Wear 194, 107-115 (1996)

10. Gohar, R., Cameron, A.: Optical measurement of oil film thickness under ehd lubrication. Nature 200, 458-459 (1963)
11. Johnston, G.J., Wayte, R., Spikes, H.A.: The measurement and study of very thin lubricant films in concentrated contacts. Tribol. Trans. 34(2), 187-194 (1991)

12. Spikes, H.A., Cann, P.M.: The development and application of the spacer layer imaging method for measuring lubricant film thickness. Proc. Instn. Mech. Engrs. Part J: J. Eng. Tribol. 215, 261-277 (2001)

13. Spikes, H.A.: The borderline of elastohydrodynamic lubrication and boundary lubrication. Proc. Instn. Mech. Engrs. Part C: J. Mech. Eng. Sci. 214, 23-37 (2000)

14. Bhushan, B.: Introduction to Tribology. Wiley, New York (2002)

15. Crook, A.W.: The lubrication of rollers. Philos. Trans. R. Soc. Lon. A 250(981), 387-409 (1958)

16. Dyson, A., Naylor, H., Wilson, A.R.: The measurement of oilfilm thickness in elastohydrodynamic contacts. Proc. Instn. Mech. Engrs. Part 3B 180, 119-134 (1965)

17. Johnson, K.L.: Contact Mechanics. Cambridge University Press, Melbourne, Australia (1987)

18. Sellgren, U., Björklund, S., Andersson, S.: A finite element-based model of normal contact between rough surfaces. Wear 254, 1180-1188 (2003)

19. Furey, M.J.: The formation of polymeric films directly on rubbing surfaces to reduce wear. Wear 26, 369-392 (1973)

20. Chaiyasit, W., Elias, R.J., Mcclements, D.J., Decker, E.A.: Role of physical structures in bulk oils on lipid oxidation. Crit. Rev. Food Sci. Nutr. 47, 299-317 (2007)

21. El-Shami, S.M., Zaki Selim, I., El-Anwar, I.M., Hassan El-Mallah, M.: Dielectric properties for monitoring the quality of heated oils. J. Am. Chem. Soc. 69(9), 872-875 (1992)

22. Galvin, G.D., Naylor, H., Wilson, A.R.: The effect of pressure and temperature on some properties of fluids of importance in elastohydrodynamic lubrication. Proc. Instn. Mech. Engrs. Part 3N 178, 283-290 (1963)

23. Onsager, L.: Electric moments of molecules in liquids. J. Am. Chem. Soc. 58, 1486-1493 (1936)

24. Valisko, M., Boda, D.: Relative permittivity of polar liquids. Comparison of theory, experiment and simulation. J. Phys. Chem. B 109, 6355-6365 (2005)

25. Dowson, D., Higginson, G.R.: Elastohydrodynamic lubrication. Pergamon Press, Oxford (1966)

26. Wong, P.L., Wang, R., Lingard, S.: Pressure and temperature dependence of the density of liquid lubricants. Wear 201(1), 58 63 (1996)

27. Tammann, G.: The dependence of the volume of solutions on pressure. Z. Phys. Chem. Stoechiom. Verwandtschafts 17, 620 636 (1895)

28. Bair, S.: Rheology and high-pressure models for quantitative elastohydrodynamics. Proc. Instn. Mech. Engrs. Part J: J. Eng. Tribol. 223(4), 617-628 (2009)

29. Acosta, G.M., Smith Jr, R.L., Arai, K.: High-pressure PVT behavior of natural fats and oils, trilaurin, triolein, and n-tridecane from $303 \mathrm{~K}$ to $353 \mathrm{~K}$ from atmospheric pressure to $150 \mathrm{MPa}$. J. Chem. Eng. Data 41, 961-969 (1996) 


\title{
The Growth of Thin Lubricating Films of Plant Oils
}

\author{
Wenhsi Chua $\cdot$ Gwidon W. Stachowiak
}

Received: 10 October 2010/ Accepted: 22 November 2010

(C) Springer Science+Business Media, LLC 2010

\begin{abstract}
The extreme conditions of high pressure and shear imposed in a lubricated sliding contact could influence tribochemical reactions that could occur over long sliding distances and time scales, possibly leading to changes in both friction and film thickness. Experiments conducted with 12 plant oils reveal for the first time, that thin lubricating films of some plant oils can grow to thicknesses much greater than what is predicted from either elastohydrodyamic theory or their adsorbed molecular heights. Some films grew as much as 25 times in thickness (unrefined canola oil), while others remained roughly unchanged (flaxseed and olive oil), or grew slightly and then collapsed during the test (safflower oil). The absence of a loss in film thickness and the viscoelastic-like behavior of the film when speeds are reduced to zero, support the view that polymerization could be the main mechanism of film growth. However, the lack of correlation between the degree of unsaturation and the film growth rate suggests that other mechanisms could also be at work.
\end{abstract}

Keywords Vegetable oils - Boundary lubrication friction - Gas chromatography $\cdot$ Acidity $\cdot$ Stick-slip

\section{Introduction}

Before mineral oils became the mainstream lubricants used today, plant oils and animal fats were used by mankind, even before recorded history. Evidence of their use in prehistoric times can be found in an Egyptian tomb that dated

W. Chua $(\varangle) \cdot$ G. W. Stachowiak

Tribology Laboratory, Department of Mechanical Engineering, University of Western Australia, Nedlands, WA 6009, Australia e-mail: whchua@mech.uwa.edu.au back to $1400 \mathrm{BC}$, where beef or mutton fat was discovered on the axles of chariots [1]. It was not until the first successful oil drilling in 1859 by "Colonel" E. L. Drake, and the soaring supply and demand of this "rock oil" which followed thereafter [1,2], that oils derived from plants and animals for use as lubricants soon became redundant. However, increased environmental awareness in recent times has provided a stimulus for the replacement of mineral-based lubricants with biodegradable and non-toxic alternatives, particularly in total-loss lubricant applications, such as in two-stroke engine oils, chain saw oils, and metal-cutting fluids $[3,4]$. Biodegradable synthetic lubricants based on esters, polyalkylene glycol (PAG), and low viscosity polyalphaolefins (PAO2) are available on the market, but are 3-30 times more costly [3, 5]. These contributing factors have spurred a revival of plant oils as base oils for lubricants.

Compared to mineral oils, plant oils have high flash points, excellent biodegradability, and are non-toxic. In addition, they possess good boundary lubrication properties and a high viscosity index. Such tribological performances can only be matched by mineral base oils, through the blending of additives. The weaknesses of plant oils, however, lie in their poor oxidation stability and cold flow properties, which can be remedied to some extent, by the use of additives or chemical modification $[3,6]$.

Plant oils are mainly composed of triglycerides which are esters of a carboxyl group (glycerol molecule) and three long chain fatty acids. The fundamental reason for their good boundary lubrication properties stems from the fact that the carboxyl group of the triglyceride is relatively polar and will adhere to metallic surfaces. They thus have the ability to assemble on the surface, with their fatty acid chains being "combed" in the direction of shear. The current understanding of its boundary lubrication mechanism is 
thus similar to that of pure fatty acids: the polarity of the triglyceride determines its adsorption strength to the surface, while the fatty acid chain lengths and degree of unsaturation determines the oil's ability to maintain a thicker and more densely packed monolayer on the surface [7].

In general, the elastohydrodynamic lubrication (EHL) theory of a linear $\log -\log$ relationship between film thickness and velocity is obeyed at the lowest speeds until films approach the dimensions of the adsorbed boundary layer. Partial evidence of plant oil boundary films can thus be found in Biresaw's EHL study of plant oils, where a deviation from linearity was observed at low-entraining speeds $[6,8]$. Of particular interest is the observation that this deviation began at higher than expected entraining velocities and at film thicknesses much greater than the triglyceride's known molecular lengths. This suggests the presence of a transition phase above that of the immobile boundary layer. The transition between EHL and boundary lubrication is sometimes referred to as the intermediate lubrication or thin film lubrication. This lubrication regime is an interesting field of study, since the dynamics of these films is governed, not only by the bulk physical laws of viscous flow and contact pressure, but also by surface forces and intermolecular interaction [9].

Under the high pressures of a sliding contact, plant oil films may undergo a rapid transition from a liquid-like to a glassy or amorphous state, where the molecules are still in the disordered state but are frozen as a result of the rapid compression [9]. Given the propensity of fatty acid chains to align in the direction of shear, it would be reasonable to suggest that they could possibly adopt more solid-like or crystalline forms. Whether such rapid thermodynamic compressions and shear could have an influence on the tribochemical reactions between the adsorbed lipid molecules and the rubbing surfaces, and between the molecules themselves, is largely unknown. If the molecular structure of the film is altered as a result of tribochemical reactions, the film forming ability and friction would also likely be affected. These changes are of critical importance in practice, since machines are generally lubricated over long periods of time.

Although state-of-the-art tribological test methods, such as the surface force apparatus (SFA) [10] and the spacer layer imaging method (SLIM) [11] provide an unprecedented level of accuracy in studying the thickness and friction of films at the nanoscale, they generally do not facilitate the study of these films over long sliding distances. A study of the influence of metallic surfaces on tribochemical reactions is also not possible, since these methods rely on the use of transparent surfaces, such as mica or glass, for film thickness measurements using optical interferometry technique. Investigations into the long-term effects of metallic sliding on both friction and thickness of thin lubricating films are thus rarely conducted.

To investigate these effects, a new ball-on-disc tribometer was recently developed at the Tribology Laboratory of the University of Western Australia. The apparatus (Figs. 1, 2), described in detail earlier [12], is capable of simultaneously measuring the film thickness and friction of boundary layers subjected to a pure sliding contact between a metallic ball and disc pair. The method of film thickness measurement is based on the method first introduced by Crook [13] and then further developed by Dyson et al. [14]. In an earlier pilot study conducted using this technique, the film thickness of refined sunflower oil was observed to grow to 2-3 times its initial thickness, but collapsed after sliding for several hours [12]. In this work, we report the results for three other refined plant oils and nine unrefined plant oils and provide a plausible explanation to these observations.

\section{Experimental Details}

The materials and test conditions were kept the same as that described in detail earlier in [12]. Briefly, the pure sliding contact was made between a stationary $8 \mathrm{~mm}$ diameter ball and a rotating $60 \mathrm{~mm}$ diameter disc, both of which are made of 52100 steel. The ball used was a commercially available bearing ball having a rated hardness of 63-65 HRB. The disc was heat-treated to a final hardness of $814 \pm 10 \mathrm{HV}$, and then polished to a mirror finish of $13 \pm 2 \mathrm{~nm} \mathrm{Ra}$, as measured using the AltiSurf optical profilometer. The oil to be tested was allowed to slowly warm to room temperature, except for palm olein, which needed to be heated in a water bath at $40{ }^{\circ} \mathrm{C}$ until it fully melted. Approximately $15 \mathrm{ml}$ of the oil was then poured into the lubricant cell so that the disc was fully immersed, and then left to sit for $1 \mathrm{~h}$ at the test temperature. The test duration was set at $18 \mathrm{~h}$, equivalent to 10800 disc revolutions. Friction was measured using a full-bridge strain gauge system on the cantilever load arm (spring constant $=2.9 \mathrm{~N} / \mathrm{mm}$ ) at a sampling rate of 1000 samples $/$ s. Capacitance and resistance measurements were simultaneously acquired using a GW Instek LCR-821 meter at the excitation frequency of $200 \mathrm{kHz}$ and sampling interval of $0.896 \mathrm{~s}$, where the bearing ball and disc served as the negative and positive electrode, respectively. Both friction and impedance measurements were averaged over a period of $6 \mathrm{~s}$, which in this work, was the time taken for one complete disc revolution. A digital microscope positioned above the disc, captured video frames of the wear track at 30 min intervals during the entire test period. The capacitance data acquired were then used to calculate the film thickness using the calculation method described in [12]. 
Fig. 1 Schematic illustration of the experimental setup [12]

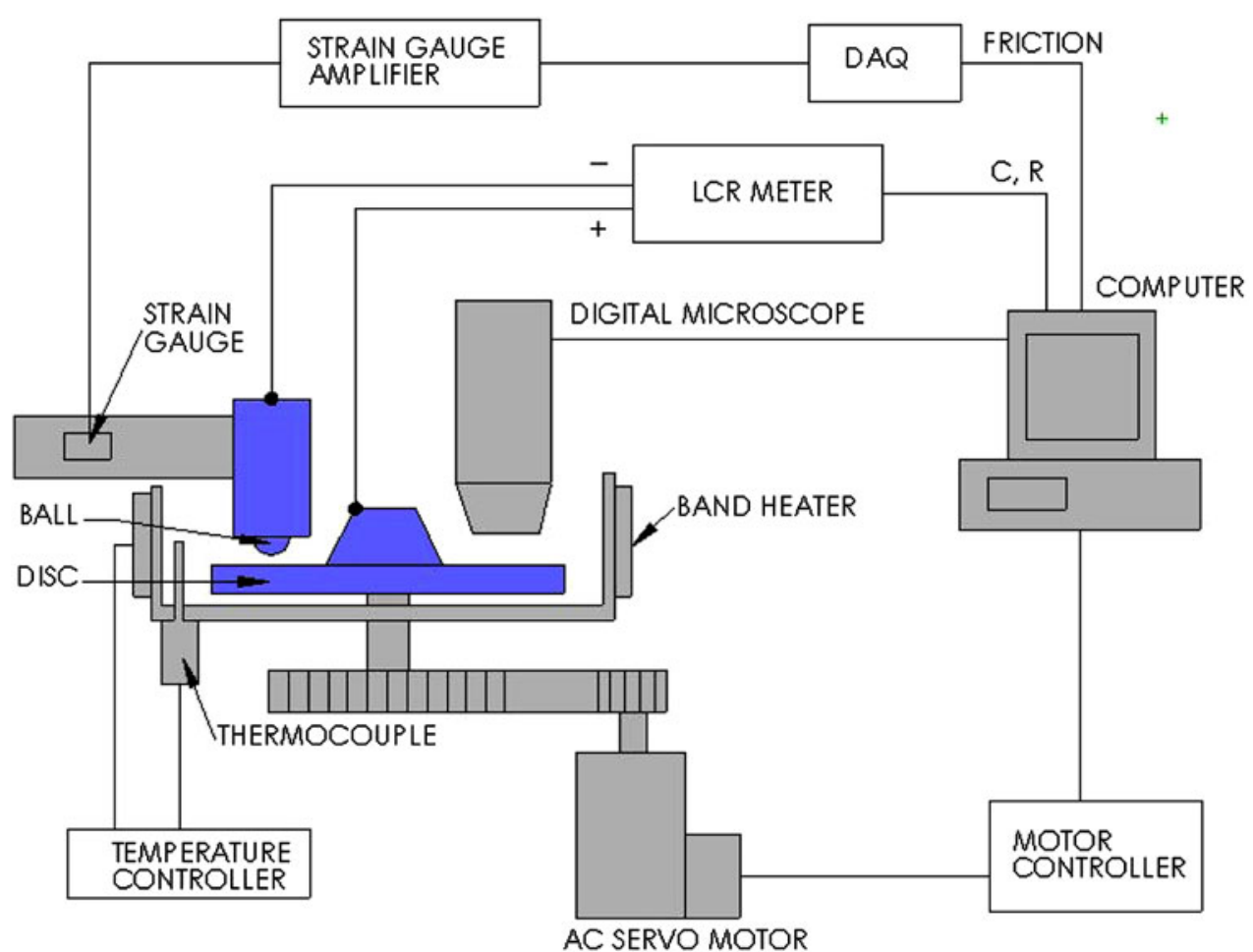

viscosity coefficients of plant oils at $40{ }^{\circ} \mathrm{C}\left(7-11 \mathrm{GPa}^{-1}\right)$ [7], the theoretical EHL film thickness would be approximately between 3 and $6 \mathrm{~nm}$ at the test speed. In this work, the polished steel disc and bearing ball have a combined surface roughness of approximately $16 \mathrm{~nm}$. The film thickness in the apparent area of contact would thus vary by an amount roughly equivalent to the combined surface roughness of the two surfaces. It should be noted that, although the capacitance method provides only an average value of the film thickness across the contact, it has the advantage of being inherently biased toward the minimum film thickness (at the asperities) when the film thicknesses are in the order of the surface roughness.

\section{Test Lubricants}

Three refined oils—canola oil, high oleic sunflower oil, and palm olein were obtained from Cargill Inc. Nine cold pressed and unrefined oils-sunflower oil, high oleic sunflower oil, safflower oil, canola oil, sesame oil, extra virgin olive oil, macadamia oil, wheatgerm oil, and flaxseed oil were purchased from Melrose Health. All the oils, except for flaxseed oil, have been stored at approximately $4{ }^{\circ} \mathrm{C}$ in the refrigerator. Due to its poor oxidation stability, flaxseed oil was stored in the freezer at approximately $-20^{\circ} \mathrm{C}$. All the oils were tested as they were, without further modification or filtration. 
Calculation of the film thickness from capacitance data requires that the dielectric constant at the average contact pressure and temperature to be known. This was calculated using empirical methods and the knowledge of the lubricant's density and refractive index, which are more conveniently measured under ambient pressures. The temperature at the contact was assumed to be the same as the bulk oil, since the sliding speed used in this work was low $(0.02 \mathrm{~m} / \mathrm{s})$.

\subsection{Density, Viscosity, and Refractive Index}

The density was measured by weighing the mass of the oil in a $10 \mathrm{ml}$ graduated volumetric flask, which was previously calibrated with distilled water. The density at the average contact pressure of $0.7 \mathrm{GPa}$ was predicted using the Tait-Tamman equation, with constants $B=110$ and $C=0.07325$, as described in [12]. The dynamic viscosities were measured using the Brooksfield Cone/Plate Digital Viscometer at shear rates of $45,22.5$, and $11.25 \mathrm{~s}^{-1}$ and then averaged. The refractive indices were measured using an Abbe Refractometer, as per AOAC Official Method 921.08 [15]. All measurements were made at the temperature of $40{ }^{\circ} \mathrm{C}$ under ambient pressures. The density at $0.7 \mathrm{GPa}$ and refractive index determined were later used to calculate the dielectric constant at the same pressure.

\subsection{Dielectric Constant}

The measurement procedure and calculation method for the dielectric constant are the same as that already described in [12], except that the ball, instead of the guard ring, was used as the negative electrode. Although this effectively reduces the precision of the measurement due to smaller measurable capacitances, it significantly reduces the measurement error due to fringing capacitances at the edges of the guard ring. Parasitic capacitances from the connecting leads are automatically offset from the measurement by the LCR meter's compensation procedure, but fringing capacitances are dependent on the permittivity of the medium to be measured [16]. The capacitance setup was thus calibrated using liquids of known dielectric constants [17] at $40{ }^{\circ} \mathrm{C}$. Five liquids-cyclohexane, chlorobenzene, toluene, 1-octanol, and glycerol, of which the first two are standard calibration liquids, were used. The dielectric constants at $0.7 \mathrm{GPa}$ were then calculated using the Onsager equation, as described in [12].

\subsection{Fatty Acid Composition and Free Fatty Acids} (FFAs)

The fatty acid compositions were determined using gas chromatography mass spectroscopy (GCMS). $3 \mu \mathrm{l}$ of the oil sample was dissolved in $150 \mu \mathrm{l}$ of toluene and $300 \mu \mathrm{l}$ of methanol with $2 \% \quad \mathrm{H}_{2} \mathrm{SO}_{4}$ was then added. The mixture was then refluxed at $80{ }^{\circ} \mathrm{C}$ for $2 \mathrm{~h}$ to produce the fatty acid methyl esters (FAMEs). $300 \mu \mathrm{l}$ of $0.9 \% \mathrm{NaCl}$ solution and $300 \mu \mathrm{l}$ of hexane were then added. The mixture was briefly shaken and then centrifuged for $3 \mathrm{~min}$ at $13200 \mathrm{rpm}$. A Pastuer pipette was then used to remove the hexanic layer for analysis in GCMS. Nonadecanoic acid (C19:0) solution was used as an internal standard for the quantification of the FAMEs.

The GCMS was performed on a 7890A gas chromatography instrument, equipped with a 122-7032 DB-Wax capillary column $(30 \mathrm{~m} \times 0.25 \mathrm{~mm}$ I.D. $\times 0.25 \mu \mathrm{m}$ film thickness) and coupled with a 5975C inert XL mass selective detector (Agilent Technologies, Little Fall, NY, USA) and a MPS 2XL autosampler (Gerstel, Mülheim an der Ruhr, Germany). Helium was used as the carrier gas at a flow rate of $1.03 \mathrm{~mL} / \mathrm{min}$. $1 \mu \mathrm{L}$ of the sample was injected applying the following conditions: injector temperature $-250{ }^{\circ} \mathrm{C}$; split ratio-1:10; column temperaturefrom $50{ }^{\circ} \mathrm{C}$ (hold time $=1 \mathrm{~min}$ ), to $200{ }^{\circ} \mathrm{C}$ at $25^{\circ} \mathrm{C} / \mathrm{min}$ and then to $230{ }^{\circ} \mathrm{C}$ (hold time $=18 \mathrm{~min}$ ) at $3{ }^{\circ} \mathrm{C} / \mathrm{min}$. The temperature of the transfer liner was set at $250{ }^{\circ} \mathrm{C}$. The mass spectrometer was operated at the ionization energy of $70 \mathrm{eV}$ and mass scanning was performed from 50 to $600 \mathrm{amu}$.

Amount of free fatty acids (FFAs) in the oils were determined by the AOAC Official Method 940.28 [15]. Iodine value was calculated using Eq. 2 in [18], from the fatty acid compositions determined by GCMS. The iodine value provides a measure of the oil's degree of unsaturation.

\section{Experimental Results}

\subsection{Physical Properties and Fatty Acid Composition}

The physical properties measured are listed in Table 1. These results are in agreement with those obtained from literature [19] in that oils with a higher degree of unsaturation are less viscous, and have higher densities, refractive indices, and dielectric constants. The plots of the oils' refractive indices, dielectric constants, viscosities, and degrees of unsaturation against their densities are shown in Fig. 3. A notable outlier for the viscosity-density relationship is wheatgerm oil. This is most likely due to the high level of insoluble matter present in the oil.

The results of the GCMS and fatty acid determination are shown in Table 2. Wheatgerm oil has the highest level of FFAs among the oils tested, which is typical of most unrefined wheatgerm oils. Flaxseed oil differs from the other highly polyunsaturated oils in that a large proportion 
Table 1 Physical properties. All properties are determined at $40{ }^{\circ} \mathrm{C}$ and ambient pressure, unless stated otherwise

\begin{tabular}{llllll}
\hline Plant oil & $\begin{array}{l}\text { Density, } \rho \\
(\mathrm{g} / \mathrm{cm})\end{array}$ & $\begin{array}{l}\text { Dielectric } \\
\text { constant, } \varepsilon\end{array}$ & $\begin{array}{l}\text { Refractive } \\
\text { index, } n\end{array}$ & $\begin{array}{l}\text { Predicted dielectric } \\
\text { constant, } \varepsilon \text { at } 0.7 \mathrm{GPa}\end{array}$ & $\begin{array}{l}\text { Dynamic } \\
\text { viscosity, } \\
\eta(\mathrm{cP})\end{array}$ \\
\hline $\begin{array}{l}\text { Refined, bleached, and deoderised oils } \\
\text { Canola oil }\end{array}$ & 0.905 & 3.00 & 1.470 & 3.15 & 32.6 \\
High oleic sunflower oil & 0.900 & 2.93 & 1.466 & 3.07 & 36.39 \\
Palm olein & 0.901 & 3.00 & 1.462 & 3.17 & 35.8 \\
Unrefined, cold-pressed oils & & & & & 27.78 \\
Sunflower oil & 0.909 & 3.05 & 1.472 & 3.21 & 36.39 \\
High oleic sunflower oil & 0.900 & 3.00 & 1.467 & 3.16 & 33.46 \\
Canola oil & 0.904 & 3.02 & 1.469 & 3.18 & 32.23 \\
Sesame oil & 0.904 & 3.01 & 1.469 & 3.16 & 28.22 \\
Safflower oil & 0.909 & 3.14 & 1.472 & 3.32 & 36.8 \\
Extra virgin olive oil & 0.899 & 2.98 & 1.465 & 3.14 & 35.62 \\
Macadamia oil & 0.901 & 2.96 & 1.464 & 3.12 & 23.65 \\
Flaxseed oil & 0.916 & 3.11 & 1.477 & 3.29 & 36.8 \\
Wheatgerm oil & 0.916 & 3.13 & 1.474 & 3.31 & \\
\hline
\end{tabular}

of its polyunsaturated fatty acids (PUFAs) is composed of $\omega$-3 fatty acids (C18:3). It was also interesting to note that the highly monounsaturated macadamia oil has the highest concentration of long chain fatty acids with more than 20 carbon atoms, and also the second highest amount of FFAs among the oils tested.

\subsection{Film Thickness and Friction}

The friction and film thickness traces were averaged over three repeat experiments, as shown in Fig. 4. For comparison, the mean film thicknesses of the first and final hour of the tests are shown in the bar chart of Fig. 5. It is clear from these results that the film thicknesses of some oils do not remain constant when subjected to continuous sliding contact. Some films grew as much as 25 times in thickness (unrefined canola oil), while others remained roughly the same (flaxseed and olive oil), or grew slightly and then collapsed during the test period (safflower oil). It would also appear that there is a maximum thickness that the films would grow to and this maximum thickness is unique to each oil. While there is a distinct relationship between the properties and the degree of unsaturation of the oils, the relationship between the latter and the film growth is less clear.

The coefficient of friction (COF) for the oils tested is in the range of $0.08-0.12$. At the start of sliding, the COF generally could be as high as 0.14 , but quickly decreased to a stable value with sliding time. With the exception of palm olein, the initial film thicknesses of the other oils tested are in the range of $4-10 \mathrm{~nm}$. It is uncertain why palm olein formed a significantly thicker film than the other oils during the initial stages, but we believe that this might be due to the fact that the test temperature of $40{ }^{\circ} \mathrm{C}$ was relatively near its solidification temperature range. Since surface boundaries are the preferred initiation sites for crystallization, some of the more saturated triglycerides that were physically adsorbed could have formed crystallites on the surfaces during the $1 \mathrm{~h}$ pre-test immersion period.

Highly polyunsaturated oils such as flaxseed and safflower oils generated very thin films $(<10 \mathrm{~nm})$, with the latter collapsing after $400 \mathrm{~min}$ of sliding. The film growth and collapse observed in safflower oil was similar to that observed in the pilot study [12] conducted using refined sunflower oil. Interestingly, although the fatty acid composition of unrefined sunflower oil is very similar to that of safflower oil, the former grew steadily to a thickness of approximately $40 \mathrm{~nm}$, without any sign of film breakdown. For wheatgerm oil, the film thickness increased quickly to approximately $30 \mathrm{~nm}$ within 3-4 h, but varied unstably between 15 and $30 \mathrm{~nm}$ thereafter.

From the results obtained, it appears that the high oleic oils, while being more stable, exhibited slower film growth. The film thickness of unrefined and refined high oleic sunflower oil grew and stabilized at approximately 12 and $15 \mathrm{~nm}$, respectively. The film thickness of palm olein peaked at $30-40 \mathrm{~nm}$ after about $6 \mathrm{~h}$ of sliding, but decreased and then stabilized at about $18 \mathrm{~nm}$. On the other hand, there was almost no change in the thickness of the film generated by olive oil throughout the entire test. Among the high oleic oils, macadamia oil exhibited the most stable film growth up to approximately $29 \mathrm{~nm}$ by the end of the test. This oil was observed to grow even up to $60 \mathrm{~nm}$ in one test. It is uncertain whether this was due to the increased presence of FFAs in the oil. 

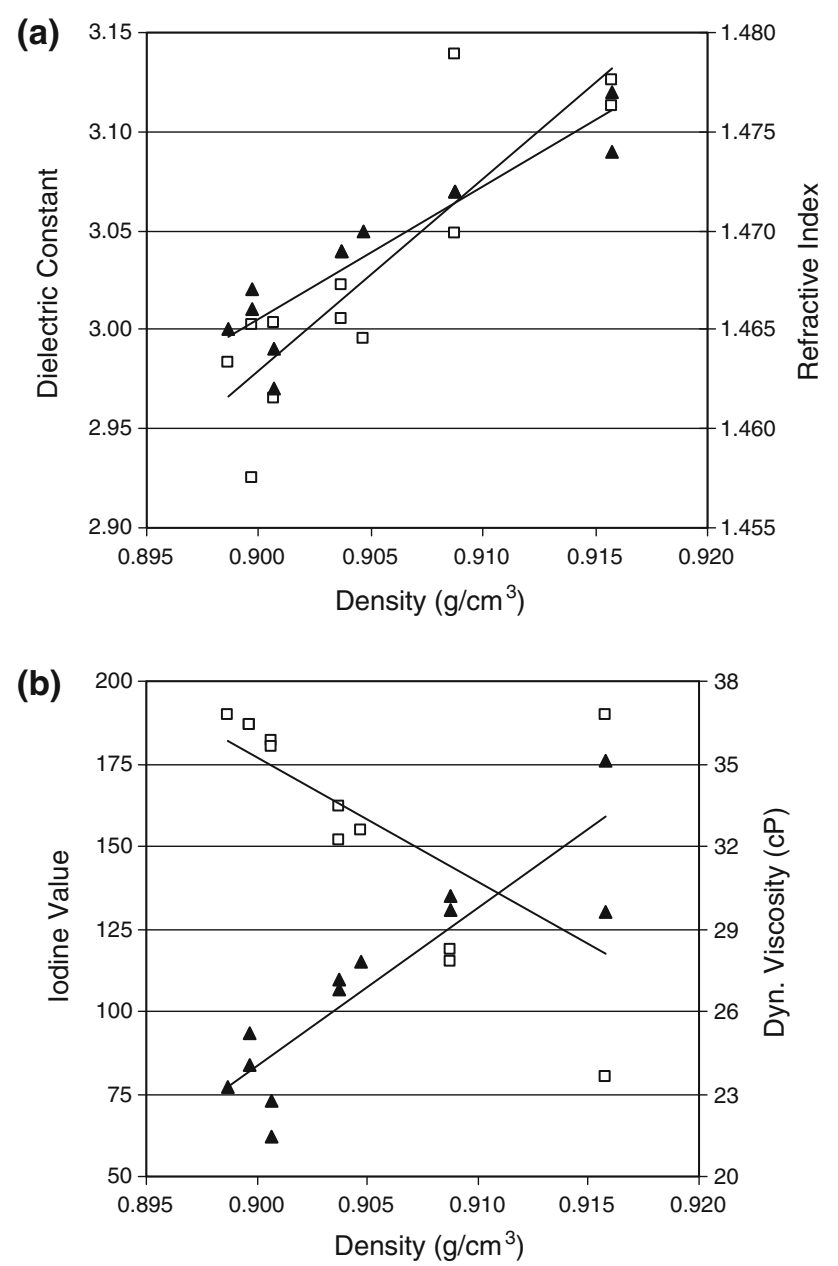

Fig. 3 a Dielectric constant (open square) and refractive index (filled triangle) vs. density; b Iodine value (filled triangle) and dynamic viscosity (open square) vs. density

The most surprising results are those of two moderately unsaturated oils-unrefined sesame oil and unrefined canola oil, where their film thicknesses increased to 50-60 $\mathrm{nm}$ and $80-130 \mathrm{~nm}$, respectively. Among the oils tested, the fastest growth rate has been that of unrefined canola oil. Its growth to approximately $100 \mathrm{~nm}$ was completed in about 6-7 h. It should be noted that refined canola oil, which has a similar fatty acid composition as its unrefined variant, but only half its acidity, did not exhibit the same film thickening behavior.

\subsection{Ball Wear Scars and Wear Tracks}

Figure 6 shows the optical microscope images taken of the ball wear scars and wear track. Except for high oleic sunflower oil (refined) and safflower oil, the diameter of the ball wear scars for all the other oils were very close to the calculated Hertzian contact diameter. On the other hand, the breakdown of the film for safflower oil caused accelerated wear to occur and this is evident in the wear scars which were more than three times larger than the others. Some scratches can also be observed on the ball wear scars of palm olein and wheatgerm oil, which can be attributed to the higher amount of insoluble matter that was present in the oils. The reddish brown color observed on the wear tracks, particularly of unrefined oils, appears to be of some corrosion products caused by chemical attack. Surface profile measurements taken after the tests for these oils reveal that there was no significant change in the surface roughness, indicating that the visible scars are mainly due to chemical, and not mechanical wear. This is consistent with the lack of any increase in the wear scar diameters from the calculated Hertzian contact diameters. For the case of safflower oil, where a film collapse occurred during the test, the change in the apparent area of contact had no effect on the film thickness calculation, since capacitances were not measurable after the film breakdown.

At higher magnifications of the ball wear scars of unrefined oils, patches could be seen distributed randomly on the wear scar (Fig. 7). It should be noted that these patches remained adsorbed on the surface, even after washes with hexane and ethanol. The optical interference effect observed on these patches is a clear indication that these patches were organic in nature. Similar patches were, however, not visible on the wear tracks.

\subsection{Transition to Stick-Slip}

Of the oils tested, canola oil, sunflower oil, and sesame oil (all unrefined oils) had the highest film growth rate. On closer examination, it appears that these oils experienced a relatively abrupt increase in the COF roughly from 0.1 to 0.12 during film growth and this increase in friction seemed to occur when their films grew thicker than $20 \mathrm{~nm}$ (see circled areas in Fig. 4b, c). Observation of the actual friction traces during each disc revolution indicates that after this occurred, friction changed from a "smooth" type to a "stick-slip" type (Fig. 8). As duly noted by Israelachvilli and co workers [10, 20], the occurrence and behavior of the stick-slip friction is dependent on the mass and stiffness of the friction force measuring system. Since this transition to stick-slip occurred without any change in the tribological conditions applied, a change in the physical state of the film must have occurred, possibly from being more liquid-like to either an amorphous or solid-like structure. The argument that this was due to metallic welding of the asperities is rejected, since a stable film of at least $20 \mathrm{~nm}$ had already developed between the surfaces, when this transition occurred.

The increase in friction with film thickness was, however, not observed for all the oils tested. The inverse was 
Table 2 Fatty acid composition and free fatty acids

\begin{tabular}{|c|c|c|c|c|c|c|c|c|c|c|c|c|}
\hline & \multicolumn{3}{|c|}{ Refined oils } & \multicolumn{9}{|c|}{ Unrefined cold-pressed oils } \\
\hline & Canola & $\begin{array}{l}\text { HO } \\
\text { Sunflower }\end{array}$ & $\begin{array}{l}\text { Palm } \\
\text { Olein }\end{array}$ & Sunflower & $\begin{array}{l}\text { HO } \\
\text { Sunflower }\end{array}$ & Canola & Sesame & Safflower & Olive & Macadamia & Flaxseed & Wheatgerm \\
\hline $\mathrm{C} 12$ & 0.00 & 0.00 & 0.29 & 0.00 & 0.00 & 0.00 & 0.00 & 0.00 & 0.00 & 0.05 & 0.00 & 0.00 \\
\hline $\mathrm{C} 14$ & 0.08 & 0.08 & 0.83 & 0.08 & 0.07 & 0.09 & 0.09 & 0.11 & 0.04 & 0.62 & 0.06 & 0.10 \\
\hline $\mathrm{C} 15$ & 0.02 & 0.02 & 0.04 & 0.02 & 0.02 & 0.02 & 0.01 & 0.02 & 0.01 & 0.02 & 0.03 & 0.05 \\
\hline $\mathrm{C} 16$ & 4.79 & 4.96 & 33.88 & 6.80 & 4.68 & 5.94 & 10.90 & 7.20 & 13.90 & 9.00 & 5.49 & 16.83 \\
\hline $\mathrm{C} 16: 1$ & 0.17 & 0.10 & 0.16 & 0.04 & 0.11 & 0.24 & 0.13 & 0.06 & 0.73 & 16.18 & 0.05 & 0.17 \\
\hline $\mathrm{C} 16: 2$ & 0.02 & 0.00 & 0.00 & 0.00 & 0.00 & 0.00 & 0.00 & 0.00 & 0.00 & 0.00 & 0.00 & 0.02 \\
\hline $\mathrm{C} 17$ & 0.06 & 0.05 & 0.10 & 0.06 & 0.04 & 0.07 & 0.06 & 0.04 & 0.06 & 0.04 & 0.07 & 0.04 \\
\hline $\mathrm{C} 18$ & 2.84 & 4.23 & 5.54 & 5.49 & 3.93 & 3.19 & 5.89 & 3.70 & 3.65 & 4.25 & 5.53 & 1.38 \\
\hline C18:1 & 56.93 & 79.47 & 45.05 & 19.97 & 70.60 & 55.58 & 39.53 & 19.52 & 72.95 & 58.95 & 21.64 & 17.05 \\
\hline $\mathrm{C} 18: 2$ & 23.29 & 8.88 & 13.18 & 65.70 & 18.67 & 23.93 & 41.41 & 68.14 & 6.91 & 2.37 & 18.30 & 54.38 \\
\hline $\mathrm{C} 18: 3$ & 9.70 & 0.13 & 0.17 & 0.17 & 0.09 & 7.19 & 0.29 & 0.09 & 0.61 & 0.14 & 48.20 & 7.70 \\
\hline $\mathrm{C} 20$ & 0.53 & 0.33 & 0.40 & 0.30 & 0.30 & 0.75 & 0.76 & 0.40 & 0.57 & 3.32 & 0.17 & 0.12 \\
\hline C20:1 & 1.01 & 0.25 & 0.18 & 0.15 & 0.23 & 1.57 & 0.31 & 0.22 & 0.33 & 3.19 & 0.15 & 1.57 \\
\hline C20:2 & 0.07 & 0.00 & 0.00 & 0.02 & 0.00 & 0.09 & 0.01 & 0.03 & 0.00 & 0.00 & 0.05 & 0.18 \\
\hline $\mathrm{C} 22$ & 0.26 & 1.10 & 0.10 & 0.92 & 0.86 & 0.52 & 0.38 & 0.32 & 0.18 & 1.03 & 0.17 & 0.11 \\
\hline C22:1 & 0.09 & 0.00 & 0.00 & 0.00 & 0.00 & 0.64 & 0.00 & 0.00 & 0.00 & 0.36 & 0.00 & 0.21 \\
\hline $\mathrm{C} 24$ & 0.14 & 0.40 & 0.08 & 0.27 & 0.39 & 0.18 & 0.22 & 0.16 & 0.06 & 0.47 & 0.11 & 0.10 \\
\hline SFA $\%$ & 8.73 & 11.17 & 41.27 & 13.94 & 10.28 & 10.76 & 18.31 & 11.95 & 18.47 & 18.81 & 11.62 & 18.74 \\
\hline MUFA\% & 58.19 & 79.82 & 45.38 & 20.17 & 70.95 & 58.02 & 39.97 & 19.80 & 74.01 & 78.68 & 21.84 & 18.99 \\
\hline PUFA $\%$ & 33.08 & 9.01 & 13.35 & 65.89 & 18.77 & 31.22 & 41.72 & 68.26 & 7.52 & 2.51 & 66.54 & 62.27 \\
\hline IV & 115.30 & 83.97 & 62.01 & 131.00 & 93.16 & 109.61 & 106.37 & 134.67 & 76.89 & 72.97 & 175.79 & 130.22 \\
\hline FFA $\%$ & 0.05 & 0.04 & 0.06 & 0.44 & 0.19 & 0.13 & 0.47 & 0.48 & 0.58 & 1.47 & 1.46 & 3.54 \\
\hline
\end{tabular}

SFA saturated fatty acids, MUFA monounsaturated fatty acids, $P U F A$ polyunsaturated fatty acids, $I V$ iodine value, $F F A \%$ free fatty acids (as $\%$ oleic acid)

true for safflower oil and refined sunflower oil (tested earlier in [12]). For these oils, the friction decreased with increasing film thickness (Fig. 9). Incidentally, the films of these oils were the only ones that broke down catastrophically during the sliding test, causing severe wear to occur. It is also uncertain why this occurred and why the frictionfilm thickness relationship for these oils are different from the other oils.

\subsection{Viscoelastic-Like Behavior at "Stop"}

An interesting observation was made when the test was stopped. When the sliding speed was reduced to zero at the end of the 18-h sliding test, the films of unrefined canola oil, sunflower oil, and sesame oil were not squeezed out from the contact, but remained thick and continued to separate the surfaces for long periods of time. It was suggested that some of these films might possibly be frozen in their solid state as long as the load is not relieved. Once the load is relieved, the molecules would tend to become disordered and liquid-like again. This was, however, not observed in the films discussed here. When the contact was unloaded for several minutes and then loaded again, there was little or no change in the thickness of the film. The same was observed even when the contact was unloaded for more than $48 \mathrm{~h}$. This indicates that the thick film did not dissolve into the bulk oil over time, but continued to be either solid-like or polymer-like, even under ambient pressures.

Furthermore, the friction did not immediately reduce to a constant value when sliding stopped, but decreased at a decreasing rate to a stable value over $2-3 \mathrm{~h}$. This timedependent behavior at "stop" bears some similarity with the viscoelastic behavior generally encountered with entangled-chain polymers. To ensure that this observation was not due to the damping characteristics of the friction force measurement system itself, the "stop" behavior was compared with the case where metallic adhesion is expected to occur-a sliding contact between rough surfaces lubricated by paraffinic oil, as shown in Fig. 10. It is clear from this comparison that the damping effect was largely due to the sunflower oil boundary film. Further experiments to investigate these properties will be conducted in the future. 
Fig. 4 Change of film thickness and friction with sliding time (upper traces and lower traces are for $\mathrm{COF}$ and film thickness, respectively). a Oils with IV < 90: Extra virgin olive oil (filled triangle); $\mathrm{HO}$ sunflower oil (refined) (filled square); Macadamia oil (filled diamond); and Palm olein (open circle); b Oils with $90<\mathrm{IV}<120$ : Canola oil (unrefined) (filled triangle); Sesame oil (filled square); Canola oil (refined) (filled diamond); and $\mathrm{HO}$ sunflower (unrefined) (open circle). c Oils with IV > 120: Wheatgerm oil (filled triangle); Sunflower oil (filled square); Flaxseed oil (filled diamond); and Safflower oil (open circle). Circled areas denote points of significant increase in friction for unrefined canola oil, sesame oil, and sunflower oil

\section{Discussion}

Initially, there was a concern that the unexpected film growth could be due to the increase of viscosity as a result of oxidation of the bulk oil. A sample of canola oil (unrefined), flaxseed oil, and high oleic sunflower oil (unrefined) were thus extracted from the lubricant cell after the 18-h test and their viscosities were tested again. There seems to be no significant change in the viscosities to suggest that this was so. Another concern was that the apparent increase in film thickness was merely due to changes in the dielectric constant as a result of chemical changes of the film. This is unlikely, since tribochemical reactions leading to oxidation processes generally increase the polarity and dielectric constant, $\varepsilon$, of the film [21]. For a constant film thickness, an increase in $\varepsilon$ of the boundary film increases the measured capacitance and thus underestimates the actual film thickness. Thus, any apparent increase in the film thickness cannot be due to an increase in $\varepsilon$. Other concerns regarding the uncertainties of the dielectric constant are further discussed in [12].

The lack of chemical attack on the ball wear scars and wear tracks of unrefined oils suggests that this chemical attack might be due to other components in the unrefined oils that are not present in the refined oils. Unrefined oils contain a high level of minor components (approx. 2-5\%) that are considerably reduced during the refining process. These include free fatty acids, trace metals, peroxides, water, phospholipids, and pigments. Some of the natural anti-oxidants such as tocopherols, sterols, phenolic compounds, and carotenoids are also destroyed or removed as a result [22]. As seen from Table 2, the amount of FFAs in unrefined oils is generally more than 10 times that found in refined oils. With the exception of wheatgerm oil, it appears that the amount of FFAs in both refined and unrefined oils is consistent with the amount of chemical attack observed on the ball wear scars and wear tracks.

FFAs are more polar than triglycerides and are able to replace physically adsorbed triglycerides on active surface sites of the metallic surface to form chemisorbed metallic soap films [23]. They are also more susceptible to autooxidation than esterified fatty acids and their presence have
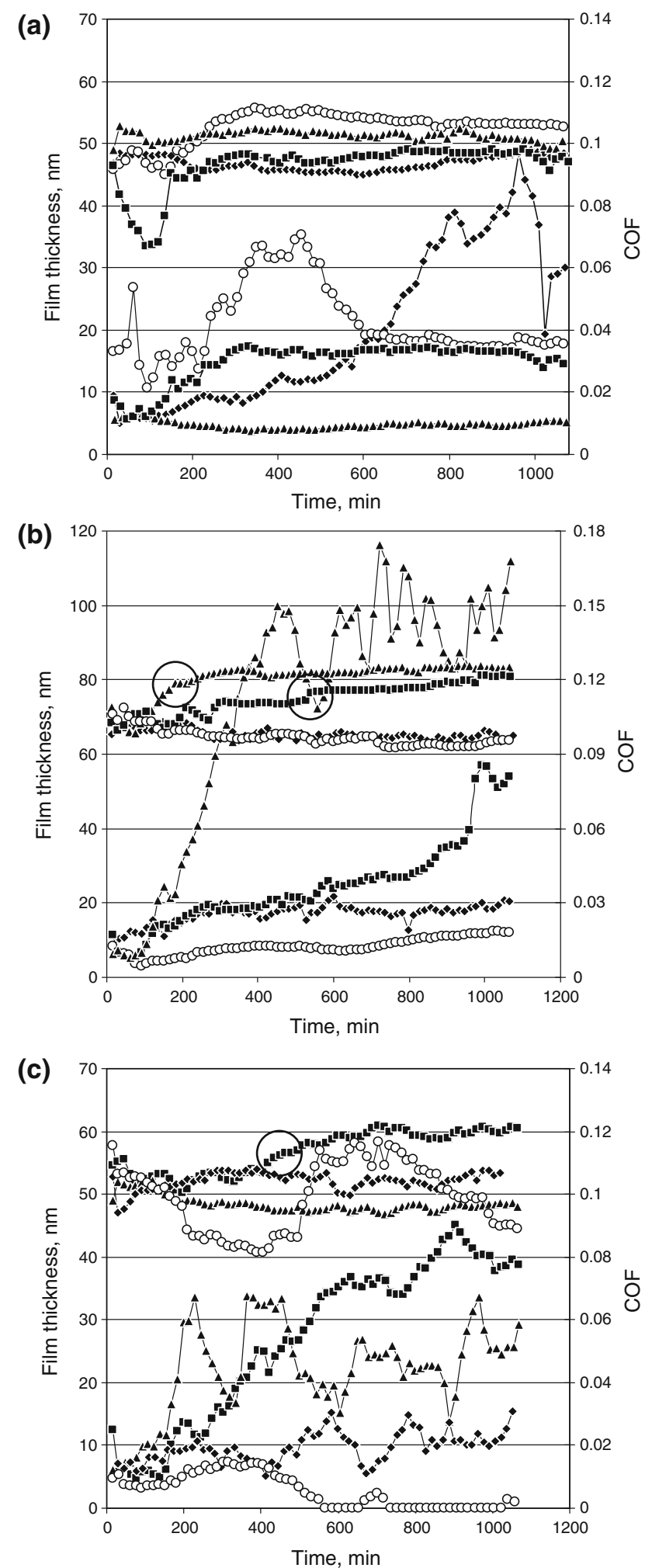

been known to accelerate the rate of oxidation in plant oils. The hydrophilic carboxyl ends of FFAs do not easily dissolve in the hydrophobic bulk oil and reside mainly at the liquid-air and liquid-solid interfaces [24]. The situation is 
Fig. 5 Bar chart showing the mean film thicknesses for the first and last hour of the sliding tests. Error bars denote the min. and max. values of the data averaged at $15 \mathrm{~min}$ intervals from three repeat experiments

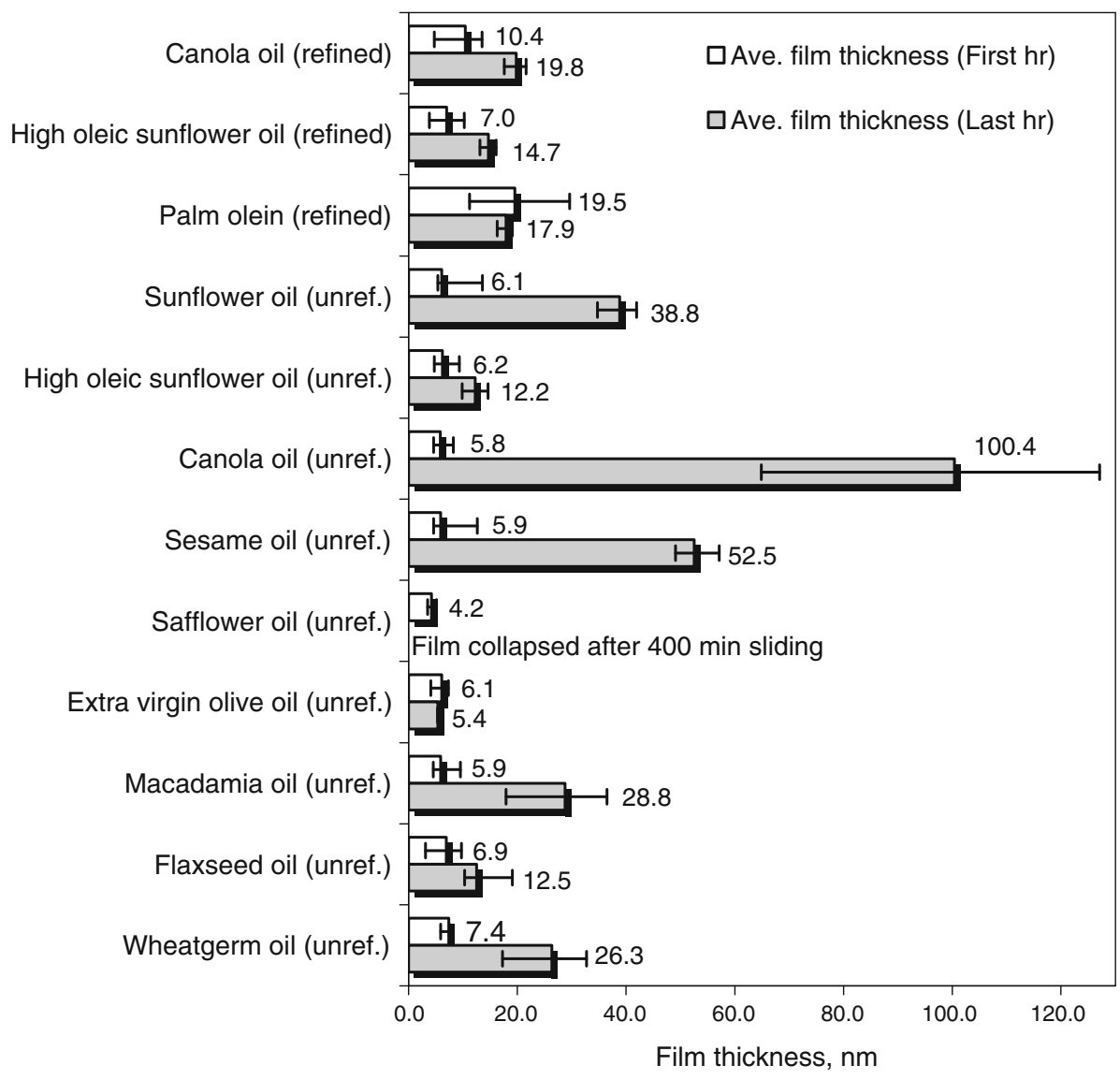

A more likely reason for the film growth would be the polymerization of the triglycerides. If oxidative processes were accelerated in the areas of sliding contact, it is highly probable that chain branching processes by polymerization could also have occurred. High pressures have been known to induce a thermodynamically unstable system that promotes chemical bonding, and have been utilized in the polymerization of unsaturated organic compounds, such as acethylene and butadiene [26]. One of the polymerization pathways for fatty acids and lipids is by addition polymerization, which occurs by the attachment of the carboxyl end of a free fatty acid to the double bond of another fatty acid chain [19, 24]. This produces high molecular weight polymeric compounds that would have much higher polarities than their parent triglycerides. The presence of iron catalysts, water, high pressures, and temperatures are known to favor the polymerization process [24]. Incidentally, these conditions are inherently prevalent in a metalto-metal sliding contact. A polymerization process that was concentrated at the areas of sliding contact would thus explain the thickening of the film. This would also explain the viscoelastic-like behavior and the sustainability of the confined films when speeds are reduced to zero.

If the rate of polymerization increases with a greater number of double bonds (higher degree of unsaturation) in 
Fig. 6 Optical microscope images of a ball wear scars and b wear tracks. From left to right in descending rows: Refined oils-sunflower oil, canola oil, and palm olein; Unrefined oils-sunflower oil, high oleic sunflower oil, canola oil, sesame oil, safflower oil, olive oil, macadamia oil, flaxseed oil, and wheatgerm oil. The reference magnification bars indicating $112 \mu \mathrm{m}$ (corresponding to the calculated Hertzian contact diameter) applies to all the images, except for those of safflower oil (middle row, rightmost), where separate reference bars, denoting the same length, are shown
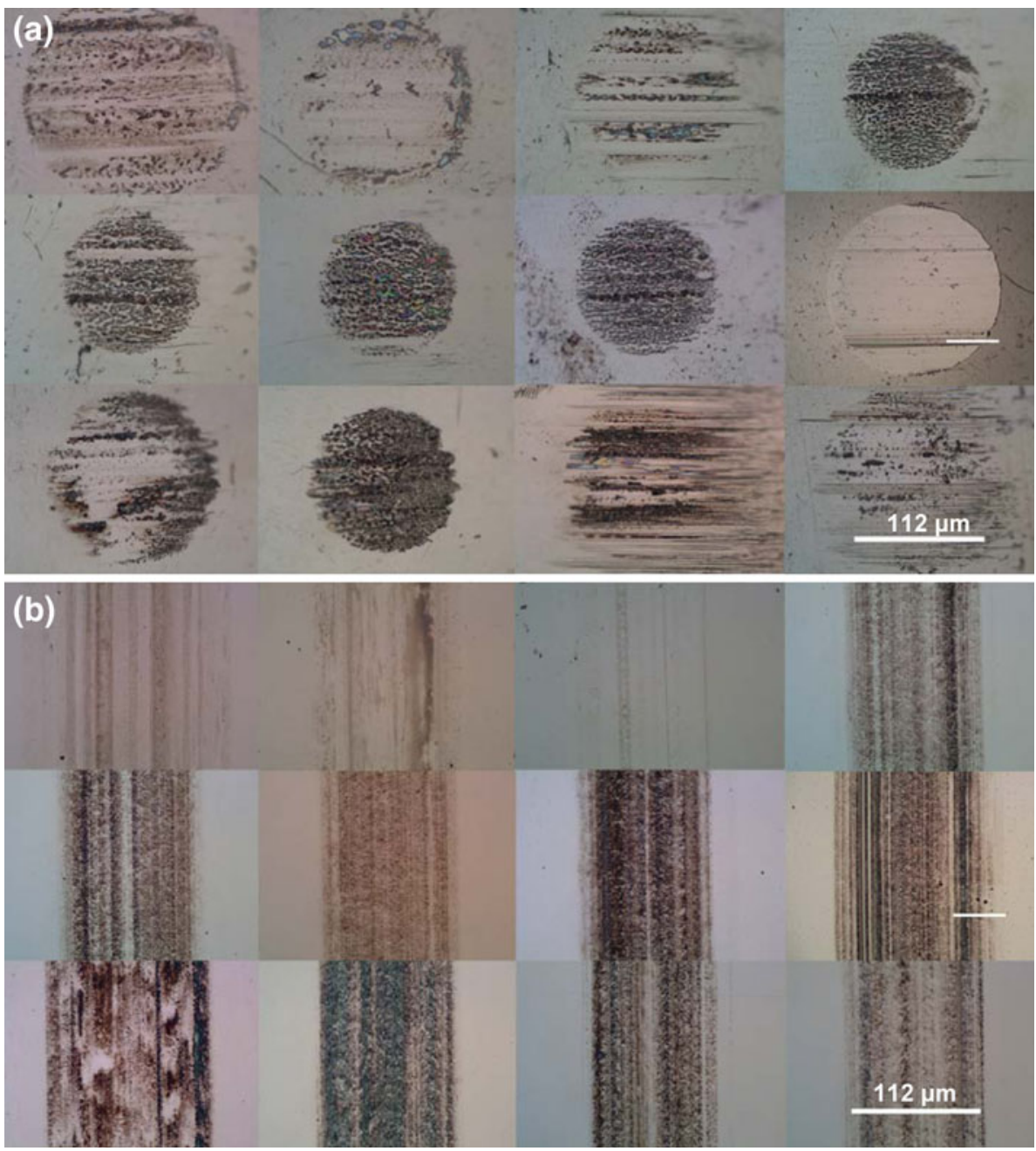

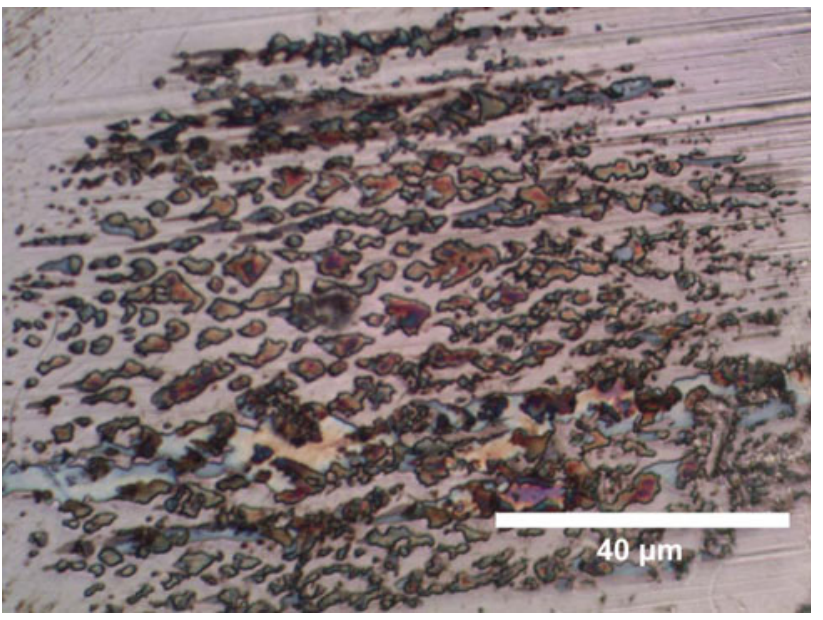

Fig. 7 Optical microscope image of the ball wear scar for unrefined sunflower oil

the plant oil, one would expect that monounsaturated oils would have poorer film growth ability than that of polyunsaturated oils. However, as previously noted, there seems to be no correlation between the film growth and the oil's unsaturation. Highly polyunsaturated oils, such as flaxseed oil, showed little film growth, while high oleic oils, such as macadamia oil, showed appreciable film growth compared to the other monounsaturated oils. Similarly, there was no significant evidence to suggest that oils with a higher amount of FFAs levels have a greater film growth rate. Unrefined canola oil, which exhibited the greatest film growth, contains the least amount of FFAs ( $0.1 \%$ oleic acid) among the unrefined oils tested. Other factors, such as the amount of water, transition metals, and anti-oxidants, which could affect the oil's tendency to polymerize, must thus be considered.

\section{Conclusions}

The chemical attack observed on the wear scars, the viscoelastic-like behavior of the film, and the absence of a reduction in film thickness when speeds are reduced to zero, support the view that polymerization of the 
Fig. 8 Friction and calculated film thickness traces for unrefined sunflower oil. Data points are averaged over one complete revolution of the disc. Insets show actual reproductions of the voltage signals from the strain gauge amplifier plotted over one disc revolution at a sampling interval of 1000 samples/s. The friction was initially smooth (inset $a$ ).

The transition to stick-slip friction occurred at approximately $20 \mathrm{~nm}$ film thickness. This transition did not occur instantly, but began over a portion of the wear track, and then gradually lengthens over several cycles until the entire wear track is of stick-slip friction (inset $b$ ). The actual voltage signal under static conditions is shown in inset $c$ for comparison

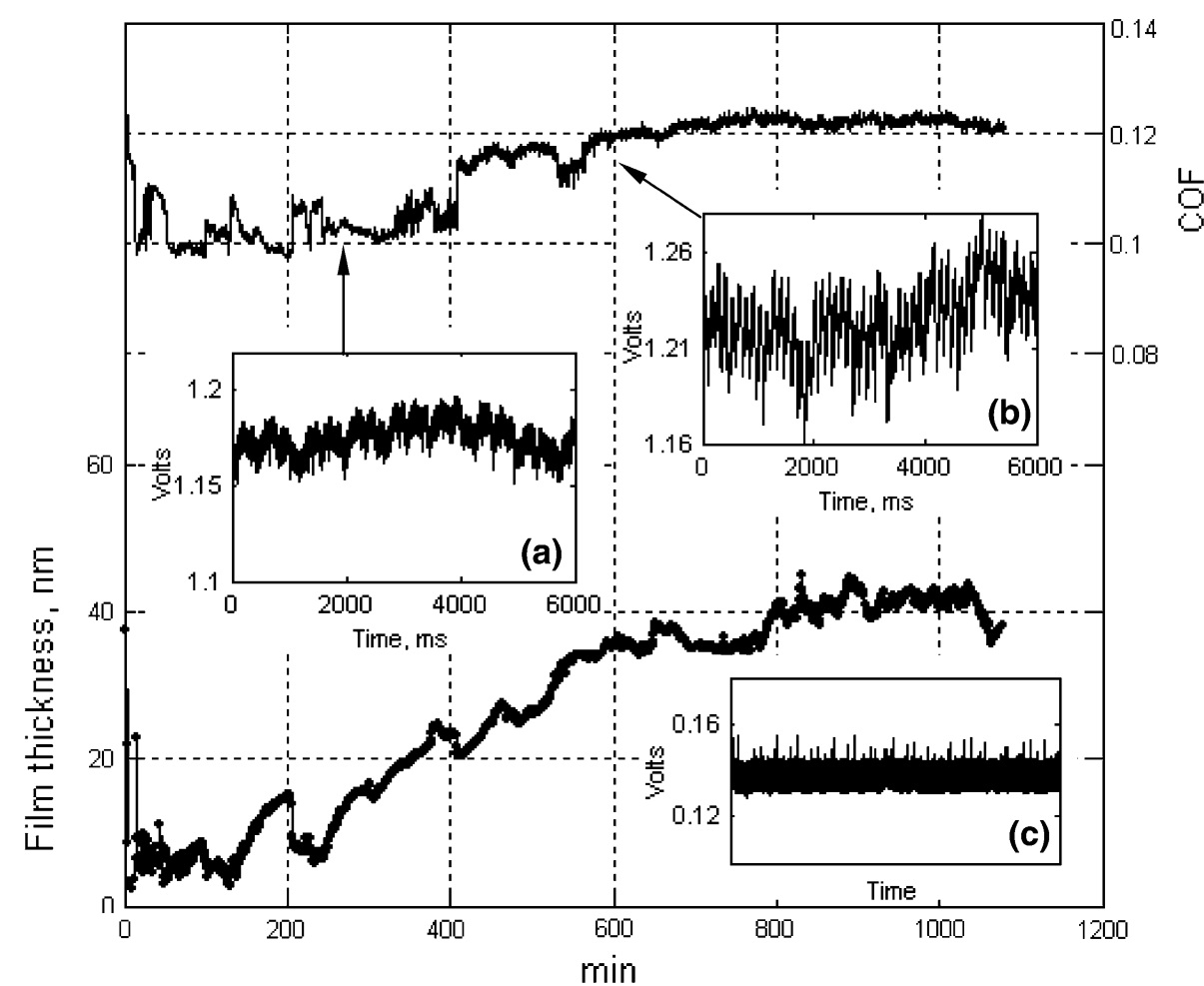

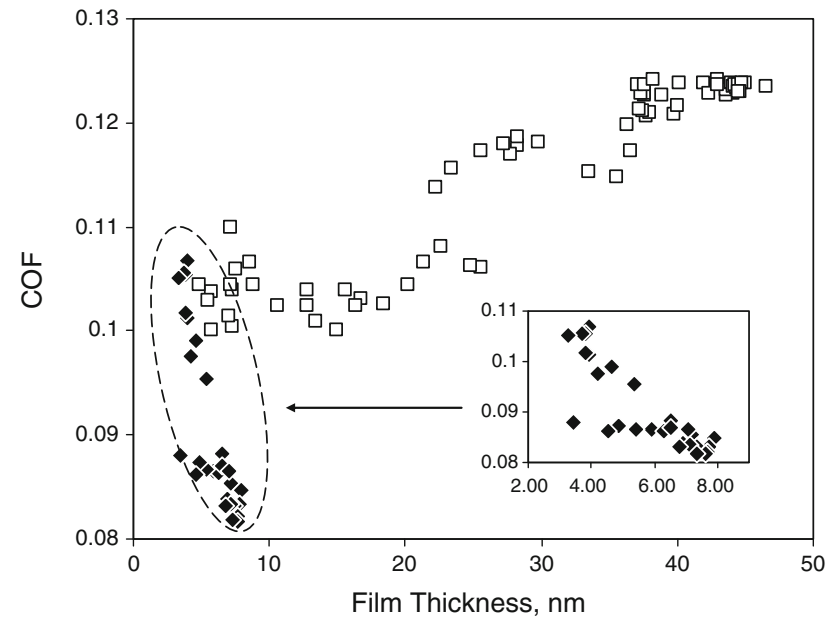

Fig. 9 Plot showing the difference in film thickness-friction relationship between unrefined safflower oil (filled diamond) and unrefined sunflower oil (open square). Inset shows the same plot for safflower oil over a smaller scale

triglycerides was the cause of the film growth. However, the lack of correlation between the growth rate and the degree of unsaturation suggests that there could be possibly other mechanisms at work. There is, however, no doubt, that the films of some oils grow to thicknesses much greater than what is predicted from either EHL theory or their absorbed molecular heights. There is also some indication that some unrefined oils could form thicker films than refined oils, and would thus possess better anti-wear

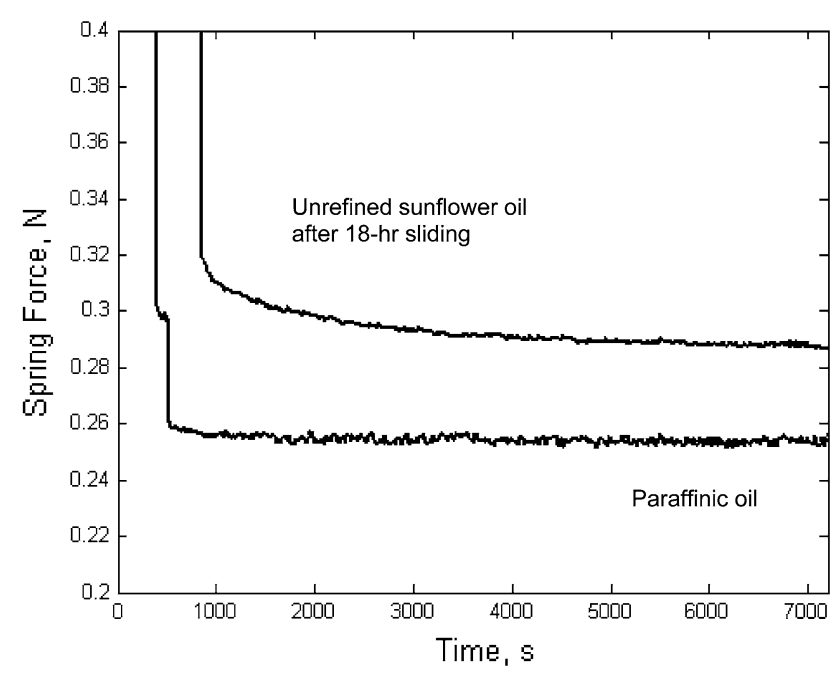

Fig. 10 Actual spring force traces when speeds are reduced to zero for paraffinic oil and unrefined sunflower oil

properties. This would be of particular interest to plant oilbased lubricant manufacturers, since a large portion of the cost is inevitably due to the refining process. The ability of some plant oil films to grow with sliding distance to thicknesses several times their molecular lengths is indeed remarkable. If the mechanisms of this film growth could be understood, plant oils with superior anti-wear properties can be developed, without excessively relying on anti-wear additives. Further studies will be conducted in the future to 
investigate the chemical structure and tribological properties of these films and to elucidate the mechanism of their growth.

Acknowledgments We thank the School of Mechanical and Chemical Engineering, University of Western Australia for its support in this work, in particular Mike Reid and Rienier de Lange for the manufacture of the test rig. We also thank Ricarda Fenske and Matthew Timmins of Metabolomics Australia, University of Western Australia, for performing the GCMS analysis. Finally, we thank Lucky Inturrisi of Cargill Inc for supplying the refined plant oils.

\section{References}

1. Dowson, D.: History of Tribology. Longman Inc., New York (1979)

2. Yergin, D.: The Prize: The Epic Quest for Oil, Money, and Power. Simon \& Schuster, New York (1991)

3. Luthur, R.: Lubricants in the environment. In: Mang, T., Dresel, W. (eds.) Lubricants and Lubrication, 2nd edn, pp. 119-182. Wiley-VCH, Weinheim (2007)

4. Bartz, W.J.: Lubricants and the environment. Tribol. Int. 31, 35-47 (1998)

5. Bartz, W.J.: Comparison of properties of synthetic fluids. In Richard Booser, E. (ed.) Tribology Data Handbook. CRC Press, Boca Raton, pp. 34-36 (1997)

6. Biresaw, G.: Elastohydrodynamic properties of seed oils. J. Am. Oil Chem. Soc. 83(6), 559-566 (2006)

7. Adhvaryu, A., Erhan, S.Z., Perez, J.M.: Tribological studies of thermally and chemically modified vegetable oils for use as environmentally friendly lubricants. Wear 257, 359-367 (2004)

8. Biresaw, G., Bantchev, G.: Effect of chemical structure on filmforming properties of seed oils. J. Synth. Lubr. 25, 159-183 (2005)

9. Yoshizawa, H., Israelachvii, J.N.: Fundamental mechanisms of interfacial friction. 2. stick-slip friction of spherical and chain molecules. J. Phys. Chem. 97, 11300-11313 (1993)

10. Homola, A.M., Israelachvili, J.N., Gee, M.L., McGuiggan, P.M.: Measurements of and relation between the adhesion and friction of two surfaces by molecularly thin liquid films. J. Tribol. 111(4), 675-682 (1989)
11. Johnston, G.J., Wayte, R., Spikes, H.A.: The measurement and study of very thin lubricant films in concentrated contacts. Tribol. Trans. 34(2), 187-194 (1991)

12. Chua, W.H., Stachowiak, G.W.: The study of the dynamic thickness of organic boundary films under metallic sliding contact. Tribol. Lett. 39, 151-161 (2010)

13. Crook, A.W.: The lubrication of rollers. Philos. Trans. R. Soc London A. 250(981), 387-409 (1958)

14. Dyson, A., Naylor, H., Wilson, A.R.: The measurement of oilfilm thickness in elastohydrodynamic contacts. Proc. Inst. Mech. Eng. Part 3B 180, 119-134 (1965)

15. AC, A.O.: Official Methods of Analysis, 15th edn. Association of Official Analytical Chemists, Inc, Washington, DC (1990)

16. Kaatze, U.: Reference liquids for the calibration of dielectric sensors and measurement instruments. Meas. Sci. Technol. 18, 967-976 (2007)

17. Maryott, A.A., Smith, E.R.: Table of Dielectric Constants of Pure Liquids (NBS Circular 514). National Bureau of Standards, New York (1951)

18. Knothe, G.: Structure indices in FA chemistry. How relevant is the iodine value? J. Am. Oil Chem. Soc. 79(9), 847-854 (2002)

19. Thomas, A.: Fats and fatty oils. In: Gerhartz, W., Yamamoto, Y.S., Kaudy, L., Rounsaville, J.F., Schulz, G. (eds.), Ullman's Encyclopedia of Industrial Chemistry, vol. A10, 5th edn. VCH Verlagsgesellschaft mbH, Weinheim, pp. 173-243 (1987)

20. Heuberger, M., Luengo, G., Israelachvili, J.N.: Tribology of shearing polymer surfaces. 1. Mica sliding on polymer (PnBMA). J. Phys. Chem. B 103, 10127-10135 (1999)

21. El-Shami, S.M., Zaki Selim, I., El-Anwar, I.M., Hassan El-Mallah, M.: Dielectric properties for monitoring the quality of heated oils. J. Am. Oil Chem. Soc. 69(9), 872-875 (1992)

22. Gunstone, F.D.: Vegetable oils. In Shahidi, F. (ed.) Bailey's Industrial Oil and Fat Products, vol. 1, 6th edn. Wiley, New Jersey, pp. 213-267 (2005)

23. Beentjes, P.C.J., Van Den Brand, J., De Wit, J.H.W.: Interaction of ester and acid groups containing organic compounds with iron oxide surfaces. J. Adhesion Sci. Technol 20(1), 1-18 (2006)

24. Choe, E., Min, D.B.: Mechanisms and factors for edible oil oxidation. Food Sci. Food Saf. 5(4), 169-186 (2006)

25. Halliwell, B., Gutteridge, J.M.C.: Free Radicals in Biology and Medicine, 3rd edn. Oxford University Press, New York (1999)

26. Schettino, V., Bini, R.: Molecules under extreme conditions: Chemical reactions at high pressure. Phys. Chem. Chem. Phys. 5, 1951-1965 (2003) 


\title{
Surface-Enhanced Raman Spectroscopy of Tribochemically Formed Boundary Films of Refined and Unrefined Canola Oils
}

\author{
Wenhsi Chua $\cdot$ Peter Chapman • \\ Gwidon W. Stachowiak
}

Received: 30 September 2011/ Accepted: 12 April 2012

(c) AOCS 2012

\begin{abstract}
The paper reports the investigation of tribochemically formed boundary films of canola oils using surface-enhanced Raman spectroscopy. This is the first time that metallic surfaces lubricated by plant oils have been studied using this technique. The results of this work provided strong evidence that fatty acids were liberated from the triglyceride structure during sliding to form a fatty acid soap layer on the silver surface. The study also revealed that the fatty acid chains of the unrefined canola oil were more disordered and most likely in a gauche conformation, while that of the refined canola oil were tightly packed and oriented perpendicular to the surface. It is believed that the greater presence of polar minor components in the unrefined oil, such as phospholipids, interfered with the ability of free fatty acids to form a tightly packed monolayer on the silver surface.
\end{abstract}

Keywords Lubricants - Fats and oils - Structurefunctional properties · Spectroscopy · Lipid chemistry · Phospholipids

\section{Introduction}

It is the widely accepted view that the boundary lubrication properties of polyunsaturated fatty acids and triglycerides

W. Chua $(\bowtie)$. G. W. Stachowiak

Tribology Laboratory, School of Mechanical and Chemical

Engineering, University of Western Australia,

Crawley, WA 6009, Australia

e-mail: whchua@mech.uwa.edu.au

P. Chapman

Department of Chemistry, School of Science,

Curtin University of Technology, Bentley, WA 6102, Australia are inferior to that of more saturated ones. This has been attributed to the double bonds that act as kinks in an otherwise straight chain fatty acid backbone, resulting in the formation of thinner and less cohesive boundary films [1-3]. However, recent experiments conducted by Murakami and Sakamoto [4] revealed that linoleic and linolenic acids were found to have more stable friction at higher temperatures. It was suggested that the polyunsaturated fatty acids formed a friction polymer that reduced friction and wear. Sahoo and Biswas [5] also found that the coefficient of friction (COF) of $n$-hexadecane doped with linoleic acid gradually reduced from 0.09 to 0.04 during sliding, while the friction remained stable when stearic acid was the additive.

The double bonds of polyunsaturated plant oils have also been widely known to be responsible for the oils' susceptibility to oxidation [6,7]. In the advance stages of oxidation, polymerization processes involving chainbranching and cross-linking mechanisms may occur. These oxidative processes could possibly be accelerated at the interface during a metallic sliding contact, resulting in the formation of thick viscous boundary films. This hypothesis was first tested in an earlier work by measuring the boundary film thickness, using the capacitance method, of four refined oils and nine cold-pressed unrefined oils in a ball-on-disk tribometer setup [8]. The tests were conducted under moderate tribological conditions of a steel-on-steel sliding contact for $18 \mathrm{~h}$. It was revealed, for the first time, that boundary film thicknesses of most plant oils do thicken over long sliding distances.

It was initially expected that the film growth could be correlated to the oil's degree of unsaturation, since a highly polyunsaturated oil would, in theory, be the most oxidatively and thermodynamically unstable, and thus possess the greatest potential for film growth. However, a fatty acid 
composition analysis using gas chromatography mass spectroscopy (GCMS) revealed that there is no such relationship. In fact, the greatest film growth (from approximately 5 to $100 \mathrm{~nm}$ ), was observed in the moderately unsaturated cold pressed canola oil. However, the boundary film of refined canola oil, which had a similar fatty acid composition, grew only to a maximum of $\sim 20 \mathrm{~nm}$. It is believed that some minor components, which are always present in unrefined plant oils, by approximately $2-5 \%[9,10]$, might have been responsible for the increased film growth.

To investigate the chemical nature of these films, which are less than $100 \mathrm{~nm}$ in thickness, it is necessary to utilize surface-sensitive spectroscopic techniques, such as grazing angle-reflectance absorption infrared spectroscopy (RAIRS) and surface-enhanced Raman spectroscopy (SERS). SERS is a powerful technique that is capable of providing molecular bonding information even from a single monolayer. The technique capitalizes on the phenomenon that the Raman signal intensity is enhanced $10^{2}-10^{6}$ times when the substrate is microscopically roughened and made of a coinage metal, such as silver, gold or copper [11]. The unique feature of SERS is that the local-field enhancement decays rapidly from the surface, so that the technique is inherently biased towards the first layer of the adsorbate. In recent times, the detection limit was found to go as low as a single molecule [12-15]. For analyzing small sample areas (less than $100 \mu \mathrm{m}^{2}$ ), the SERS method is preferred over RAIRS since conventional Raman spectrometers can achieve spot sizes down to $2 \mu \mathrm{m}$ in diameter. The disadvantage of the SERS technique, however, is that the surface needs to be made of silver, copper or gold. A clearer understanding of the chemical nature of these boundary films on transition metals must therefore await the investigation using RAIRS and its comparison with SERS results. In this work, the SERS results are presented.

\section{Experimental Section}

\section{Tribological Test and Sample Preparation}

Silver electroplating of the bearing ball was done in a local silver plating shop. The thickness of the silver coating was measured to be approximately $0.02 \mathrm{~mm}$ using a digital vernier caliper. The refined and unrefined canola oils were obtained from Cargill Inc and Melrose Health Pty Ltd, respectively. The apparatus used for the sliding experiments was an in-house developed ball-on-disc tribometer. The tribological conditions used were kept the same as that described in an earlier work [8], except that the bearing ball used was replaced with a silver coated bearing ball (Fig. 1). Briefly, the conditions were as follows: sliding speed, $0.02 \mathrm{~m} / \mathrm{s}$; temperature, $40{ }^{\circ} \mathrm{C}$; load, $7 \mathrm{~N}$; duration/total

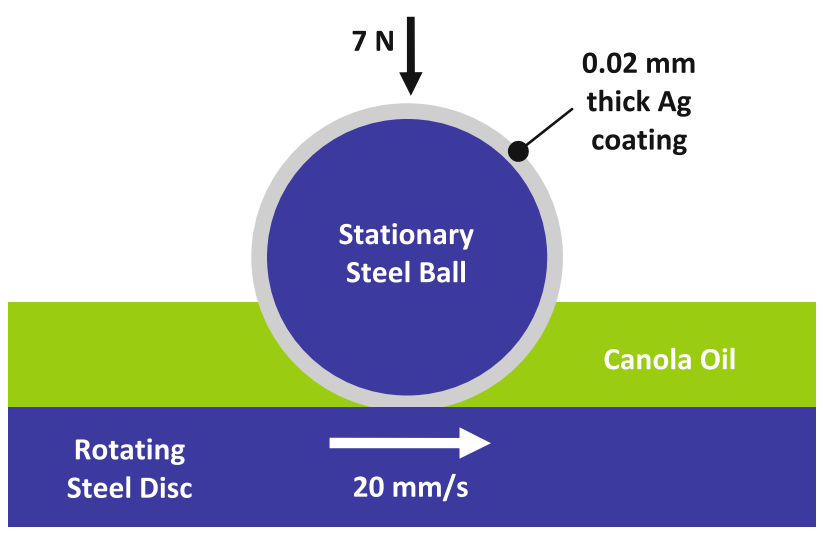

Fig. 1 Illustration of the ball-on-disc pure sliding contact under fully flooded lubricated condition

number of cycles, $18 \mathrm{~h} / 10,800$ cycles; Material (disc), 52,100 steel hardened to $810 \pm 10 \mathrm{HV}$ and polished to $13 \mathrm{~nm} \mathrm{Ra}$; Material (ball), 52,100 bearing ball ( $8 \mathrm{~mm}$ dia.) with silver coating.

After each sliding test, the silver-coated bearing ball (still wet with a layer of the bulk oil) was carefully removed from the test apparatus and transferred to a sample holder. It was then stored by immersing it in the same oil used in the test and kept at approximately $4{ }^{\circ} \mathrm{C}$ in the refrigerator. This was done to prevent any oxidation that would have otherwise occurred if the layer of oil was exposed to the atmosphere for long periods of time. SERS was conducted not more than $12 \mathrm{~h}$ from the time of storage. Prior to SERS, the sample holder with the bearing ball was removed from the bulk oil, washed briefly with hexane, and then allowed to dry under ambient conditions. The sliding experiment and SERS were also repeated to ensure reproducibility of the peaks obtained.

\section{Surface Enhanced Raman Spectroscopy}

The Raman spectrometer used was a Dilor Labram model 1B, equipped with a 30-mW He-Ne laser, coupled to an Olympus BX40 microscope. Raman spectra were recorded at different spots on the wear scar with $\sim 2.4 \mathrm{~cm}^{-1}$ resolution over a spectral range of $3,063-127 \mathrm{~cm}^{-1}$, using the $632.8 \mathrm{~nm}$ laser line via a $50 \times$ microscope objective to focus onto a spot approximately $2 \mu \mathrm{m}$ in diameter. The $520.7 \mathrm{~cm}^{-1}$ line of silicon was used for wavelength calibration. A neutral density filter (D1 filter) was used to attenuate the laser power to $10 \%(0.4 \mathrm{~mW}$ at the sample). Under such conditions, it was found that any spot on the wear scar should not be irradiated for more than $150 \mathrm{~s}$, otherwise decomposition of the film would occur. An accumulation time of $60 \mathrm{~s}$ was chosen, thus allowing for a repeat spectrum to be collected at the same spot for comparison. 


\section{Results and Discussion}

It was observed that the silver coating on the bearing ball was too soft, and was continually being sheared away during sliding. Black deposits were also observed deposited around the wear scar after the sliding tests (Fig. 2). These black deposits were later found to be amorphous carbon in the form of aggregated soot. It is clear that the film growths observed in a lubricated steel-on-steel condition were not reproduced here with the silver-coated bearing ball as the counter surface. However, the SERS spectra obtained from the two oils showed persistent and reproducible differences in their features and these differences provided the basis for comparison in this study.

Initially, SERS was conducted on the wet sample, i.e., where the surface of the ball was still covered by a thin layer of the bulk oil. The spectra obtained for the unrefined oil were dominated mainly by fluorescence (not shown), presumably

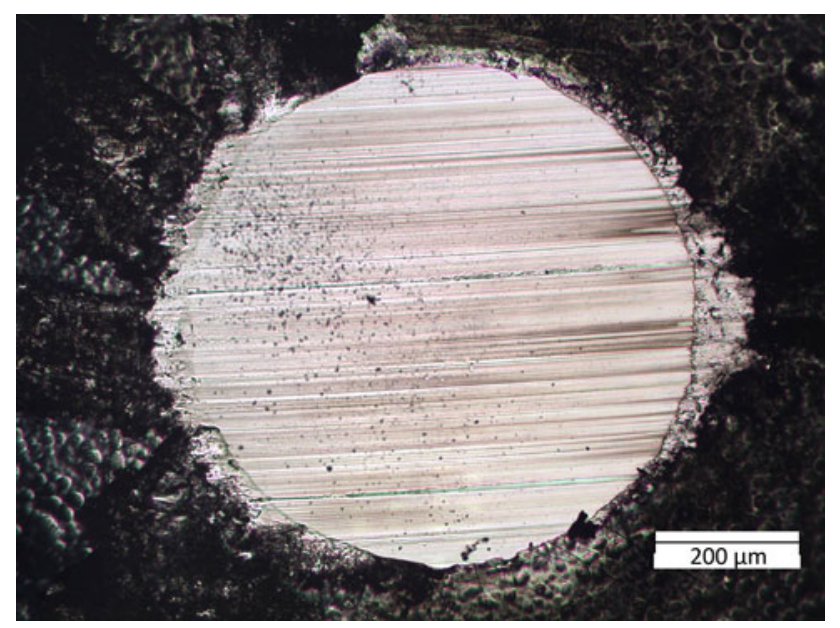

Fig. 2 Optical microscope image of the unwashed wear scar of the silver-coated ball for unrefined canola oil due to the presence of carotenoids. The fluorescence was less in the refined oils spectra, but the contribution of Raman scattering from the bulk liquid layer exceeded that that of the adsorbate in most cases. This is evident from the major bands which are characteristic of the normal Raman spectra of plant oils (Fig. 3) i.e. the $\mathrm{CH}_{2}$ and $\mathrm{CH}_{3}$ stretching modes $\left(2,800-3,000 \mathrm{~cm}^{-1}\right)$, symmetric rock in cis double bond $\left(1,265 \mathrm{~cm}^{-1}\right)$, in-phase $\mathrm{CH}_{2}$ twist $\left(1,305 \mathrm{~cm}^{-1}\right), \mathrm{CH}_{2}$ scissoring mode $\left(1,442 \mathrm{~cm}^{-1}\right)$, cis double bond stretching $\left(1,655 \mathrm{~cm}^{-1}\right)$ and ester stretching $\left(1,747 \mathrm{~cm}^{-1}\right)[16,17]$. In addition, weaker peaks near 235, 930, 1,377 and $1,560 \mathrm{~cm}^{-1}$ (labeled in figure) that are not usually typical of the normal Raman spectrum of plant oils were observed in some of the spectra collected. The first three peaks were later found to be due to the vibrations of the carboxylate groups, which were most likely enhanced due to its proximity with the silver surface. The superposition of the Raman contribution from both the bulk liquid and adsorbate made it difficult to conclude whether the vibrations originate from the bulk liquid, the adsorbate or both. These problems were avoided once the bulk oil layer was removed with a non-polar solvent.

It was observed that once the bulk oil layer was removed, many of the peaks associated with the normal Raman spectra of the bulk oil disappeared, leaving behind the contributions from the SERS effect. Assignment of the peaks identified from the spectra of both refined and unrefined canola oils are summarized in Table 1. It should be noted that the films were, however, more susceptible to the effects of photodecomposition due to excessive laser irradiation. Above $10 \mathrm{~s}$ of irradiation under the full laser power of $4 \mathrm{~mW}$, two new peaks can be observed near 1,350 and $1,600 \mathrm{~cm}^{-1}$ and their intensities increased with irradiation time. These peaks are, without doubt, identified as the $\mathrm{D}$ and G peaks of amorphous carbon (Fig. 4) [18-22] and has been similarly observed in many previous SERS studies due to either photodecomposition or carbon contamination during surface preparation $[14,15,20]$. Care was thus taken to
Fig. 3 SERS spectra of the unwashed ball wear scar of refined canola oil with enhanced vibrational bands (labeled)

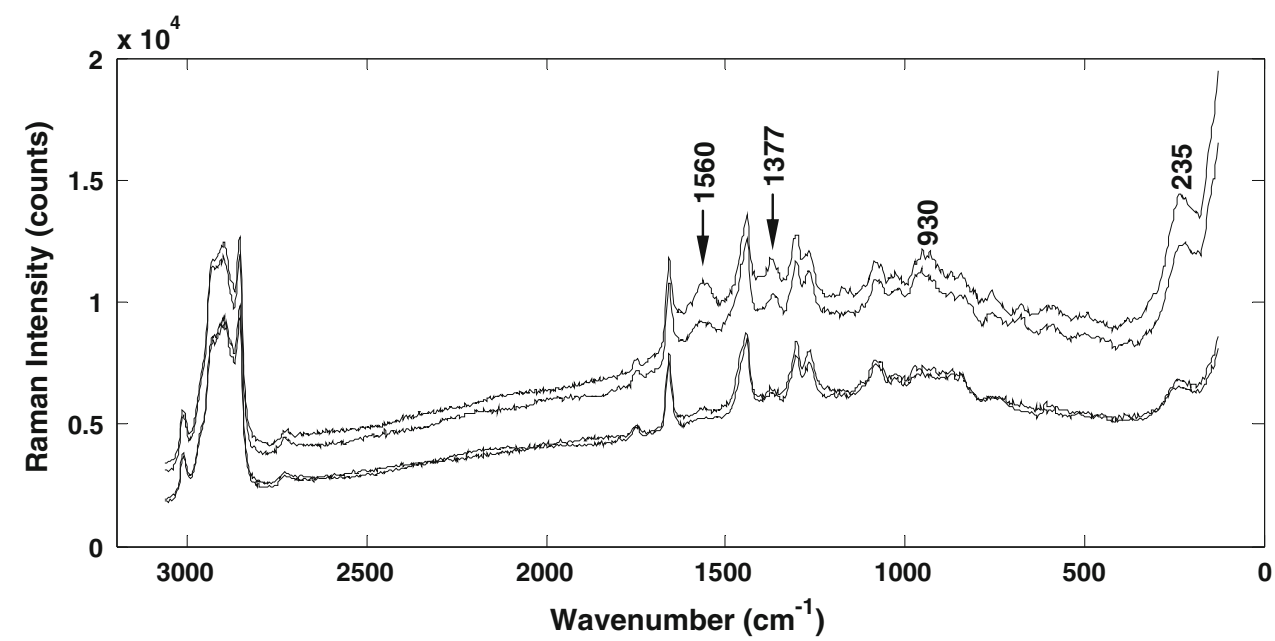

Springer AOCS * 
Table 1 Assignment of the SERS spectra peak frequencies for refined and unrefined canola oil

\begin{tabular}{|c|c|c|}
\hline \multirow[t]{2}{*}{ Vibrational band assignment } & \multicolumn{2}{|c|}{ Peak position $\left(\mathrm{cm}^{-1}\right)$} \\
\hline & $\begin{array}{l}\text { Refined } \\
\text { canola oil }\end{array}$ & $\begin{array}{l}\text { Unrefined } \\
\text { canola oil }\end{array}$ \\
\hline$v(\mathrm{Ag}-\mathrm{O})$ & 235 & 215 \\
\hline $\mathrm{CH}_{3}$ rock, $\tau(\mathrm{C}-\mathrm{H})$ & 760 & 760 \\
\hline$v(\mathrm{C}-\mathrm{C})$ & & 892 \\
\hline \multirow[t]{2}{*}{$v\left(\mathrm{C}-\mathrm{COO}^{-}\right)$} & 930 & 930 \\
\hline & 955 & \\
\hline$\delta(\mathrm{C}-\mathrm{H}), v(\mathrm{C}-\mathrm{O})$ & 1,030 & \\
\hline$v(\mathrm{C}-\mathrm{C})_{\mathrm{G},}, v\left(\mathrm{PO}_{4}^{3-}\right)$ & & 1,090 \\
\hline$\delta(\mathrm{C}-\mathrm{H}), v(\mathrm{C}-\mathrm{C})$ & 1,167 & \\
\hline$\tau\left(\mathrm{CH}_{2}\right)$ & 1,300 & 1,295 \\
\hline$v\left(\mathrm{COO}^{-}\right)$ & 1,377 & 1,395 \\
\hline$\delta\left(\mathrm{CH}_{2}\right)$ & & 1,439 \\
\hline$v(\mathrm{C}=\mathrm{C})$ aromatic ring & 1,560 & \\
\hline$v(\mathrm{C}=\mathrm{C})$ olefinic chain & 1,630 & 1,630 \\
\hline$v(\mathrm{C}=\mathrm{O})$ & 1,700 & \\
\hline$v\left(\mathrm{CH}_{2}\right)_{\mathrm{S}}$ & & 2,855 \\
\hline$v\left(\mathrm{CH}_{2}\right)_{\mathrm{A}}$ & & 2,875 \\
\hline$v\left(\mathrm{CH}_{3}\right)$ & 2,930 & 2,930 \\
\hline
\end{tabular}

$v$ stretching, $\tau$ twisting/wagging, $\delta$ bending/scissoring, ${ }_{S}$ symmetric, ${ }_{A}$ antisymmetric, ${ }_{G}$ gauche, ${ }_{T}$ trans

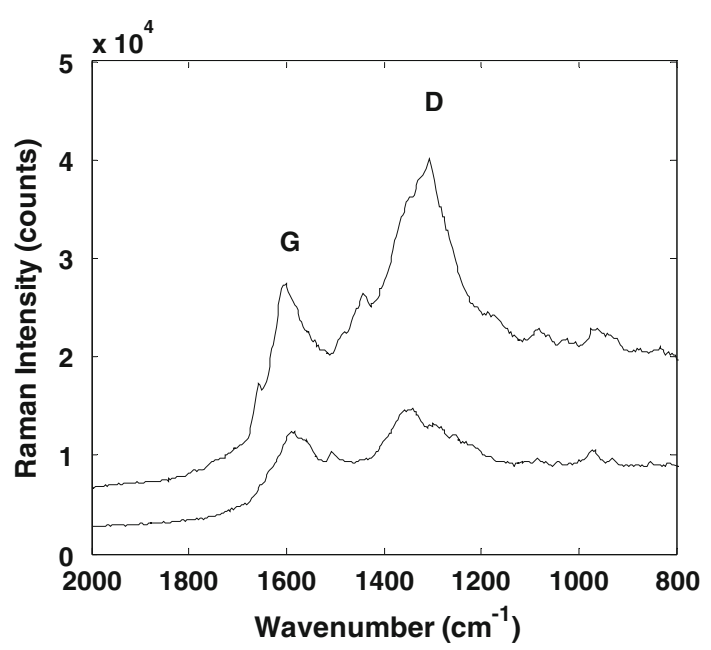

Fig. 4 SERS spectra for refined canola oil (full laser power $4 \mathrm{~mW}$; irradiation time $6 \mathrm{~s}$ )

ensure that the laser power and irradiation time was selected such that the highest intensities can be obtained without chemically altering or degrading the film.

As a comparison, SERS spectra were also collected from a new silver-coated bearing ball, which had been immersed in the refined canola oil for $18 \mathrm{~h}$ under ambient conditions. The Raman intensities of the spectra obtained were very poor, indicating that the canola oils did not form chemisorbed films with the silver surface. This is expected, since triglycerides are not known to form chemisorbed films on metallic surfaces, but are physically adsorbed onto the surface via their hydrogen bonds. This also shows that the spectra obtained from worn samples are due solely to a tribochemically formed boundary film.

\section{Refined Canola Oil}

The strongest peaks appeared near 235, 940, 1,377 and $2,930 \mathrm{~cm}^{-1}$ for the case of refined canola oil (Fig. 5a). On closer examination, it appears that the $940 \mathrm{~cm}^{-1}$ peak was in fact a doublet at 930 and $955 \mathrm{~cm}^{-1}$. The doublet (930, $955 \mathrm{~cm}^{-1}$ ) and the broad peak at $1,377 \mathrm{~cm}^{-1}$ can be assigned to the $\mathrm{C}-\mathrm{COO}^{-}$and $\mathrm{COO}^{-}$vibration of the carboxylate groups, respectively, while the peak at $235 \mathrm{~cm}^{-1}$ can be assigned to the vibration of the $\mathrm{Ag}-\mathrm{O}$ bond [23-30]. It is uncertain at this point why the doublet appears for the $\mathrm{C}-\mathrm{COO}^{-}$group. The spectra collected are similar to that reported by Moskovits and Suh [23] for mono- and dicarboxylic acids on silver sols, though the peak frequency of the $\mathrm{COO}^{-}$band was comparatively lower by about $20 \mathrm{~cm}^{-1}$. The strength of these three peaks $(235,930 / 955$ and $1,377 \mathrm{~cm}^{-1}$ ) indicates that the fatty acids were liberated from the triglyceride structure during sliding, and that it was the fatty acids themselves that were chemically adsorbed onto the silver surfaces through their carboxylate groups. This is a testament to the view first proposed by Bowden and Moore [31], who observed that lubrication using ethyl stearate on base metals was as effective as stearic acid, hence prompting them to suggest that the esters hydrolysed to form free fatty acids that reacted with the metallic surface.

The appearance of the shoulder at $1,700 \mathrm{~cm}^{-1}$, which is synonymous with the stretching of $\mathrm{C}=\mathrm{O}$ [27-29], suggests the presence of non-dissociated carboxylate groups of unadsorbed fatty acids, i.e. carboxylates groups having the $\mathrm{COOH}$ structure as opposed to $\mathrm{COO}^{-}$. For non-dissociated carboxylate groups, it has been reported that the $\mathrm{C}-\mathrm{COO}^{-}$band (normally at approx. $930 \mathrm{~cm}^{-1}$ ) would be red-shifted to $905 \mathrm{~cm}^{-1}[27,28]$ and this can be found in the spectra in the form of a shoulder at the carboxylate band. A similar red shift had also been observed for the $\mathrm{COO}^{-}$band, which might explain the slightly lower than expected peak frequency observed at $1,377 \mathrm{~cm}^{-1}$. These are strong indicators that some unadsorbed fatty acids reside at the surface, possibly stacked above the first adsorbed layer.

For the bands assigned to the vibrations of the $\mathrm{CH}_{2}$ and $\mathrm{CH}_{3}$ groups located between 3,000 and $2,800 \mathrm{~cm}^{-1}$, only a broad band centered at $2,930 \mathrm{~cm}^{-1}$, representing the Fermi resonance of the $\mathrm{CH}_{3}$ symmetric and $\mathrm{CH}_{2}$ asymmetric stretching modes, was observed (Fig. 6a). No peaks were observed at 2,850 and $2,885 \mathrm{~cm}^{-1}$ which are usually 
Fig. 5 SERS spectra $\left(2,100-100 \mathrm{~cm}^{-1}\right)$ of ball wear scar (after hexane washing) for a refined canola oil, b unrefined canola oil
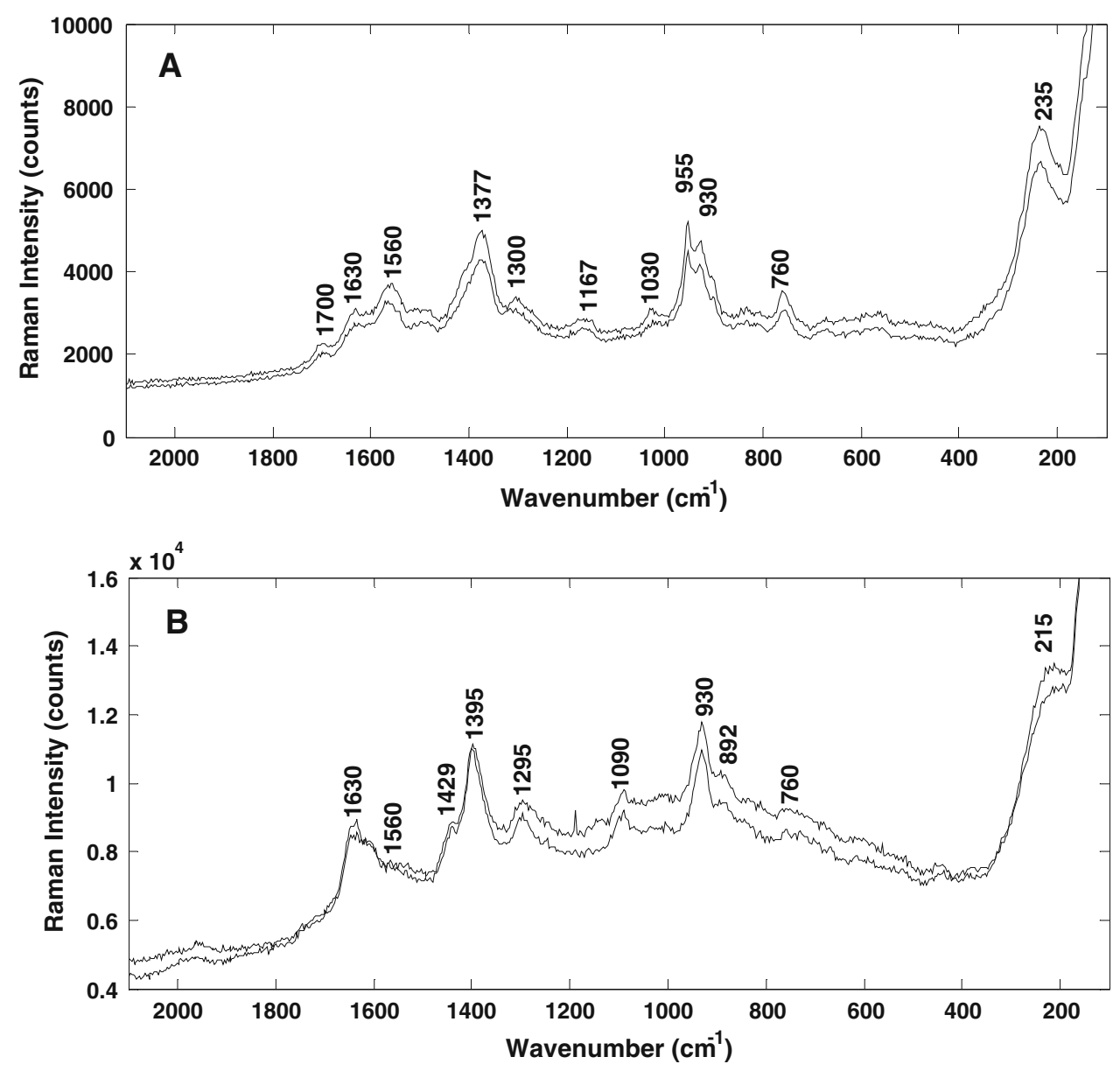

assigned to the symmetric and asymmetric stretching modes of the $\mathrm{CH}_{2}$ group [32-35]. The peak at $760 \mathrm{~cm}^{-1}$ can be assigned to either the terminal $\mathrm{CH}_{3}$ rocking mode $[36,37]$ or $\mathrm{C}-\mathrm{H}$ out of plane deformation [38]. It is interesting to note that there is a lack of contribution from the stretching vibrations of the $\mathrm{CH}_{2}$ groups since the population of $\mathrm{CH}_{2}$ monomers clearly outnumbers the terminal $\mathrm{CH}_{3}$ group for long chain fatty acids. The most plausible explanation for this is that the chains had adopted an all-trans conformation and were aligned at or close to the perpendicular axis of the surface, so that the $\mathrm{C}-\mathrm{H}$ bonds and their stretching axes were essentially parallel to the surface. Under SERS selection criteria, no enhancement of such vibrations would occur [39, 40]. Interestingly, no peaks could be found near 1,060 and $1,130 \mathrm{~cm}^{-1}$, which are normally assigned to the vibrations of all-trans segments of alkyl chains [23, 32-35].

The medium intensity peak at $1,560 \mathrm{~cm}^{-1}$ peak can also be assigned to the sp2 bonding of aromatic $\mathrm{C}=\mathrm{C}$ in amorphous carbon structures [18-22, 41], while the shoulder at $1,630 \mathrm{~cm}^{-1}$ can be assigned to the stretching vibration of the shorter $\mathrm{C}=\mathrm{C}$ of olefinic chains [26]. These two bands were observed even at the lowest irradiation times of $15 \mathrm{~s}$,

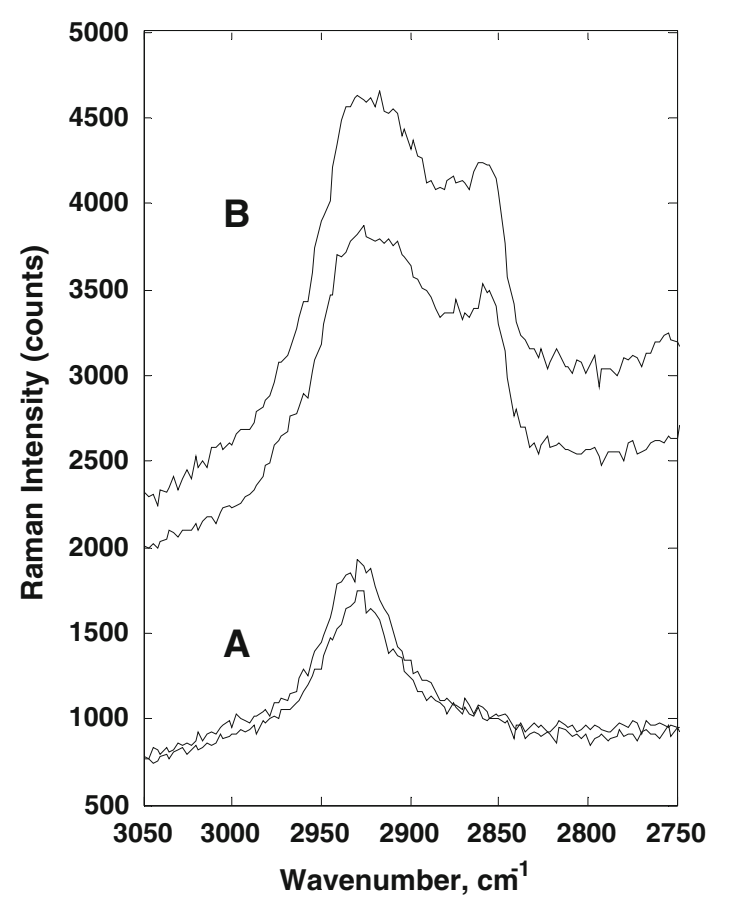

Fig. 6 SERS spectra $\left(3,050-2,750 \mathrm{~cm}^{-1}\right)$ of ball wear scar (after hexane washing) for a refined canola oil, and $\mathbf{b}$ unrefined canola oil 
which precludes the possibility that this was due to decomposition products due to excessive irradiation.

Two weaker peaks at 1,030 and $1,167 \mathrm{~cm}^{-1}$ were also noted. Assignment of these two peaks is difficult because they have been associated with several different types of molecular vibrations, including that of the $\mathrm{C}-\mathrm{H}$ in plane bending modes of aromatic compounds [25, 26, 42]. The possibility of aromatic compounds cannot be excluded since thermal oxidation of plant oils can produce cyclic and non-cyclic carbon-to-carbon linked dimers and trimers [6].

\section{Unrefined Canola Oil}

The spectra for the unrefined canola oil exhibit strong peaks at 215,930 and $1,395 \mathrm{~cm}^{-1}$, which have been similarly assigned to $v(\mathrm{Ag}-\mathrm{O}), \quad v\left(\mathrm{C}-\mathrm{COO}^{-}\right)$and $v\left(\mathrm{COO}^{-}\right)$, respectively (Fig. 5b). This indicates that the fatty acids were also liberated from the triglyceride structure to form metallic soaps with the silver surface, as in the case of the refined oil. The $\mathrm{CH}_{2}$ twisting mode near $1,295 \mathrm{~cm}^{-1}$ was also observed in the unrefined oil spectra, but with greater intensity. In contrast to the multiple peaks observed in the refined oil spectra from 1,500 to $1,700 \mathrm{~cm}^{-1}$, only a broad band peaking at $1,630 \mathrm{~cm}^{-1}$ [previously assigned to $v(\mathrm{C}=\mathrm{C})$ of olefinic chains] was observed here. Other differences include the disappearance of the weaker peaks at 1,030 and $1,167 \mathrm{~cm}^{-1}$ found in the spectra of the refined oils. Instead, a medium intensity peak appeared at $1,090 \mathrm{~cm}^{-1}$ and two shoulders appeared at 892 and $1,439 \mathrm{~cm}^{-1}$, where the latter two bands can be assigned to the alkyl terminal $\mathrm{C}-\mathrm{C}$ stretching $[26,35]$ and the $\mathrm{CH}_{2}$ bending and scissoring modes, respectively $[32,35]$.

Of notable difference is the shape of the $2,800-3,000 \mathrm{~cm}^{-1}$ band. In contrast to that of the refined oil, there is an obvious peak near $2,850 \mathrm{~cm}^{-1}$ for the SERS spectra of the unrefined oil, which has been assigned to the symmetric stretching of the $\mathrm{CH}_{2}$ group (Fig. 6b). This indicates that the boundary film of the unrefined oil was more disordered and less tightly packed, compared to that of the refined oil. The higher intensities of the $1,295 \mathrm{~cm}^{-1}$ peak and the appearance of the shoulders at 892 and $1,439 \mathrm{~cm}^{-1}$ are also indicative of the increased number of $\mathrm{CH}_{2}$ and $\mathrm{CH}_{3}$ groups that are free to undergo twisting, bending and scissoring motions. It was also noted that the high intensity of the $760 \mathrm{~cm}^{-1}$ peak (assigned to $\mathrm{CH}_{3}$ rocking) of the refined oil spectra was not similarly observed in the unrefined oil spectra, suggesting the lack of alignment of the terminal group as compared to the former. The appearance of a peak near $1,090 \mathrm{~cm}^{-1}$ is also in agreement with the shape of the $3,000-2,800 \mathrm{~cm}^{-1}$ band, since this peak is normally assigned to the gauche conformation of the $\mathrm{C}-\mathrm{C}$ skeletal backbone-a characteristic typical of highly disordered films [32, 33, 35].

The $1,090 \mathrm{~cm}^{-1}$ peak can also be associated with the molecular vibrations of phosphates of phospholipids [4345]. Indeed, ICP-MS of the canola oils revealed that there is five times more phosphorus in the unrefined oil than in the refined oil. The hydrolysis of phospholipids produces lysophospholipids, phosphate anions and amines, in addition to free fatty acids. Regardless of whether this chemical decomposition occurred during sliding, both phosphate anions and phospholipids are highly polar, and would undoubtedly compete with liberated free fatty acids for surface adsorption sites. We believe that this competitive adsorption decreases intermolecular coupling, preventing the fatty acids from forming a tightly packed boundary layer and thus imparting a degree of fluidity in the film.

The difference in the spectra obtained for refined and unrefined canola oil in the spectral region 1,500-1,650 $\mathrm{cm}^{-1}$ is also worthy of discussion. Though the $1,560 \mathrm{~cm}^{-1}$ peak (assigned to aromatic $\mathrm{C}=\mathrm{C}$ ) was not entirely absent in the unrefined oil spectra, the $1,630 \mathrm{~cm}^{-1}$ peak (assigned to
Fig. 7 Proposed model of the boundary film structure of a refined canola oil and; b unrefined canola oil

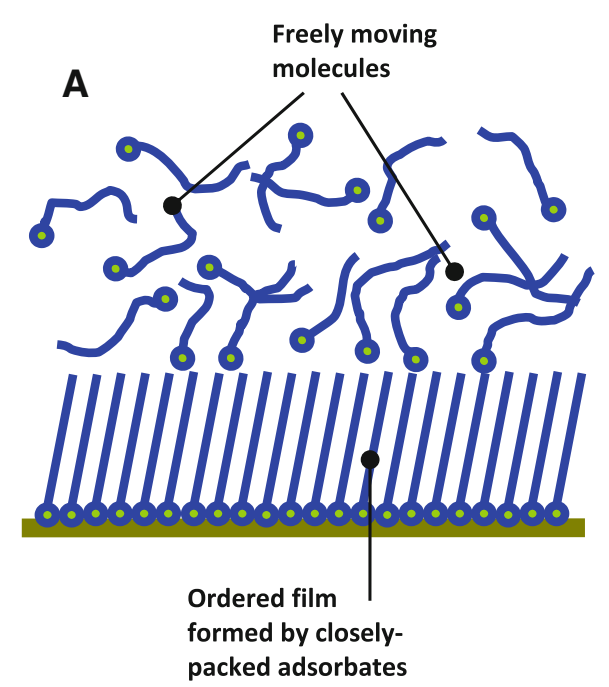

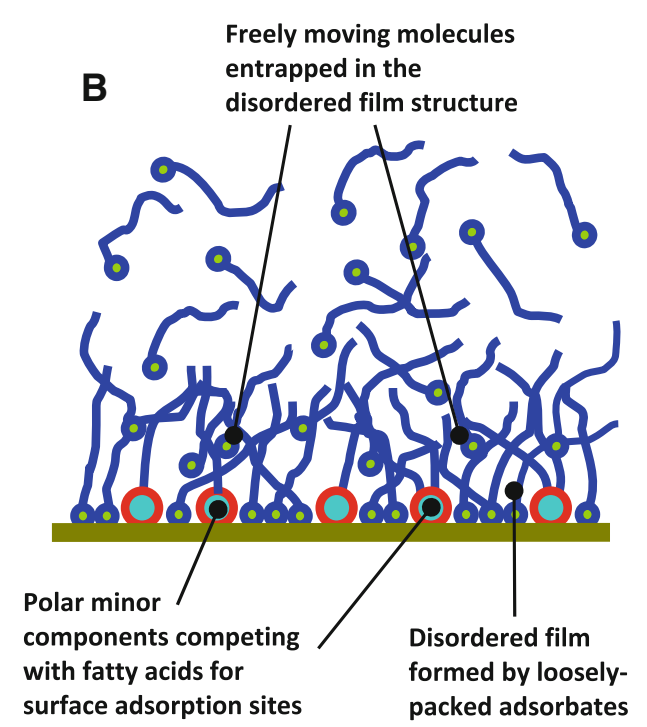


olefinic $\mathrm{C}=\mathrm{C}$ ) was always the more dominant in this spectral region. The opposite was true for the refined oil spectra. The higher intensity of the $1,630 \mathrm{~cm}^{-1}$ peak of the unrefined oil spectra may at first suggest that there was a greater population of $\mathrm{C}=\mathrm{C}$ from olefinic chains, but a more likely explanation would be that the increased intensity was due to the closer proximity of the double bonds to the silver surface. This supports the view that the chains were in a gauche conformation and were thus oriented closer to the parallel plane of the surface.

From the preceding arguments, it is clear that the tribochemically reacted films generated by the unrefined canola oil were more disordered than those formed by the refined oil. If this disorder is indeed a characteristic of the unrefined oil boundary films, the mechanism of which this disorder promotes film growth must then be elucidated. We postulate that, while an ordered and tightly packed adsorbed layer possesses higher shear strength and is more wear resistant, their inherently more crystalline and stable nature forbids their interaction with free moving molecules that are constantly being introduced into the contact area during sliding. On the other hand, when the film is sparsely packed, the chains enjoy a greater degree of freedom and interaction with free moving molecules and are hence more susceptible to chemical bonding and intermolecular chain cross-linking (Fig. 7).

\section{Conclusion}

The work presented in this paper has shown that SERS can provide a wealth of information regarding the chemical nature and surface adsorption characteristics of tribochemically formed boundary films. The analysis clearly showed that for both refined and unrefined canola oils, free fatty acids were liberated from the triglycerides under the action of sliding, and were then adsorbed onto the silver surface via their carboxylate groups. The spectra obtained also revealed that the adsorbed films of the unrefined oil were in a higher state of disorder than that of the refined oil. The more disordered nature of the unrefined oil boundary film could be explained by the competitive adsorption of polar minor components, such as phospholipids, with the free fatty acids, whereby the ability of the free fatty acids to form tightly packed films, would be impeded. It is, however, uncertain whether this disorder in the first adsorbed layer could have been one of the key requirements for film growth as observed under lubricated steel-steel condition. A more conclusive analysis would have to await further experiments using other thin film spectroscopy techniques, such as grazing angle-FTIR or sum-frequency generation (SFG) spectroscopy to probe the chemical structure of the thick films as developed under the lubricated steel-steel conditions.
Acknowledgments We thank the School of Mechanical and Chemical Engineering at the University of Western Australia for its support in the preparation of this work. We also thank the Department of Chemistry at Curtin University for the use of its vibration spectroscopy facilities.

\section{References}

1. Bhuyan S, Sundararajan S, Yao L, Hammond EG, Wang T (2006) Boundary lubrication properties of lipid-based compounds evaluated using microtribological methods. Tribol Lett 22(2):167-172

2. Lundgren SM, Ruths M, Danerlöv K, Persson K (2008) Effects of unsaturation on film structure and friction of fatty acids in a model base oil. J Colloid Interface Sci 326:530-536

3. Ruths M, Lundgren S, Danerlöv K, Persson K (2008) Friction of fatty acids in nanometer-sized contacts of different adhesive strength. Langmuir 24:1509-1516

4. Murakami T, Sakamoto H (2003) Lubricating properties of vegetable oils and paraffinic oils with unsaturated fatty acids under high-contact-pressure conditions in four-ball tests. J Syn Lubri 20(3):183-201

5. Sahoo RR, Biswas SK (2009) Frictional response of fatty acids on steel. J Colloid Interface Sci 333:707-718

6. Choe E, Min DB (2006) Mechanisms and factors for edible oil oxidation. Comp Rev Food Sci Food Safety 5(4):169-186

7. Fox NJ, Stachowiak GW (2007) Vegetable oil-based lubricantsa review of oxidation. Tribol Int 40(7):1035-1046

8. Chua W, Stachowiak GW (2011) The growth of thin lubricating films of plant oils. Tribol Lett 41:451-462

9. Gunstone FD (2005) Vegetable oils. In: Shahidi F (ed) Bailey's industrial oil and fat products, vol 1. Wiley, New Jersey, pp 213-267

10. Prior EM, Vadke VS, Sosulki FW (1991) Effect of heat treatments on canola pressed oils. I. Non-triglyceride components. J Am Oil Chem Soc 68(6):401-406

11. Moskovits M (2005) Surface-enhanced Raman spectroscopy: a brief perspective. In: Kneipp K, Moskovits M, Kneipp H (eds) Surface-enhanced Raman scattering-physics and applications, Topics in Appl. Phys, vol 103. Springer, New York, pp 1-18

12. Constantino CJL, Lemma T, Antunes PA, Aroca R (2001) Single molecule detection using surface-enhanced resonance Raman scattering and Langmuir-Blodgett monolayers. Anal Chem 73: 3674-3678

13. Kneipp K, Wang Y, Kneipp K, Perelman LT, Itzkan I, Dasari RR, Feld MS (1997) Single molecule detection using surfaceenhanced Raman scattering (SERS). Phys Rev Lett 78(9): $1667-1670$

14. Otto A (2002) What is observed in single molecule SERS, and why? J Raman Spec 33:593-598

15. Pieczonka NPW, Aroca RF (2005) Inherent complexities of trace detection by surface-enhanced Raman scattering. Chem Phys Chem 6:2473-2484

16. Baeten V, Hourant P, Morales MT, Aparicio R (1998) Oil and fat classification by FT-Raman spectroscopy. J Agric Food Chem 46:2638-2646

17. Muik B, Lendl B, Molina-Díiaz A, Ayora-Cañada MJ (2005) Direct monitoring of lipid oxidation in edible oils by Fourier transform Raman spectroscopy. Chem Phys Lipids 134:173-182

18. Ramsteiner M, Wagner J (1987) Resonant Raman scattering of hydrogenated amorphous carbon: evidence for $\pi$-bonded carbon clusters. Appl Phys Lett 51(17):1355-1357

19. Veres M, Füle M, Tóth S, Koós M, Pócsik I (2004) Surface enhanced Raman scattering (SERS) investigation of amorphous carbon. Diam Relat Mater 13:1412-1415 
20. Tsang JC, Demuth JE, Sanda PN, Kirtley JR (1980) Enhanced Raman scattering from carbon layers on silver. Chem Phys Lett 76(1):54-57

21. Schwan J, Ulrich S, Batori V, Ehrhardt H, Silva SRP (1996) Raman spectroscopy on amorphous carbon films. J Appl Phys 80(1):440-447

22. Gilkes KWR, Sands HS, Batchelder DN, Robertson J, Milne WI (1997) Direct observation of sp3 bonding in tetrahedral amorphous carbon using ultraviolet Raman spectroscopy. Appl Phys Lett 70(15):1980-1982

23. Moskovits M, Suh JS (1985) Conformation of mono- and dicarboxylic acids adsorbed on silver surfaces. J Am Chem Soc 107:6826-6829

24. Miragliotta J, Benson RC (1996) Analysis of stearic acid adsorbed on silver surface using optical spectroscopic techniques. In: Advanced applications of lasers in materials processing/broadband optical networks/smart pixels/optical MEMS and their applications. IEEE/LEOS 1996 Summer topical meetings, pp 49-50

25. Castro JL, López Ramírez MR, López Tocón I, Otero JC (2002) Surface-enhanced Raman scattering of 3-phenylproponic acid (hydrocinnamic acid). J Raman Spec 33:455-459

26. Sengupta A, Thai CK, Sastry MSR, Matthaei JF, Schwartz DT, Davis EJ, Baneyx F (2008) A genetic approach for controlling the binding and orientation of proteins on nanoparticles. Langmuir 24:2000-2008

27. Park SM, Kim K, Kim MS (1995) Adsorption of picolinic and nicotinic acids on a silver sol surface investigated by Raman spectroscopy. J Mole Struct 344:195-203

28. Królikowska A, Kudelski A, Michota A, Bukowska J (2003) SERS studies on the structure of thioglycolic acid monolayers on silver and gold. Surf Sci 532-535:227-232

29. Han B, Li Z, Wandlowski T (2007) Adsorption and self-assembly of aromatic carboxylic acids on Au/electrolyte interfaces. Anal Bioanal Chem 388:121-129

30. Thompson WR, Pemberton JE (1995) Characterization of octadecylsilane and stearic acid layers on $\mathrm{Al}_{2} \mathrm{O}_{3}$ surfaces by Raman spectroscopy. Langmuir 11:1720-1725

31. Bowden FP, Moore AC (1951) Physical and chemical adsorption of long chain compounds on radioactive metals. Trans Faraday Soc 47:900-908

32. Orendorff CJ, Ducey MW Jr, Pemberton JE (2002) Quantitative correlation of Raman spectral indicators in determining conformational order in alkyl chains. J Phys Chem A 106:6991-6998
33. Snyder RG, Strauss HL (1982) C-H stretching modes and the structure of n-alkyl chains. 1. Long disordered chains. J Phys Chem 86:5145-5150

34. Macphail RA, Strauss HL, Snyder RG (1984) C-H stretching modes and the structure of $n$-alkyl chains. 2. Long, all-trans chains. J Phys Chem 88:334-341

35. Brown KG, Bicknell-Brown E, Ladjadj M (1987) Raman-active bands sensitive to motion and conformation at the chain termini and backbones of alkanes and lipids. J Phys Chem 91:3436-3442

36. Overman SA, Thomas GJ Jr (1999) Raman markers of nonaromatic side chains in an $\alpha$-helix assembly: Ala, Asp, Glu, Gly, Ile, Leu, Lys, Ser, and Val residues of phage $f d$ subunits. Biochemistry 38:4018-4027

37. Matsuura H, Fukuhara K, Masatoki S, Sakakibara M (1991) Molecular conformation of nonionic surfactant in the solid state. A Raman spectroscopic study of a homologous series of $\alpha$-n-alkyl$\omega$-hydroxyoligo(oxyethylene)s. J Am Chem Soc 113:1193-1202

38. Xue G, Dong J, Zhang J (1991) Surface-enhanced Raman scattering of polymer on metal. 2. Molecular chain orientation of polybenzimidazole and poly $\left({ }_{\mathrm{L}}\right.$-histidine) and its transition. Macromolecules 24:4195-4198

39. Philip D, John A, Panicker CY, Varghese HT (2001) FT-Raman, FT-IR and surface enhanced Raman scattering spectra of sodium salicylate. Spectrochim Acta Part A 57:1561-1566

40. Suh JS, Michaelian KH (1987) Surface-enhanced Raman scattering as a probe of surface geometry effects on the polymerization of acrylic acid on silver. J Phys Chem 91:598-600

41. Pagannone M, Fornari B, Mattei G (1987) Molecular structure and orientation of chemisorbed aromatic carboxylic acids: surface enhanced Raman spectrum of benzoic acid adsorbed on silver sol. Spectrochim Acta Part A 43(5):621-625

42. Wei F, Zhang D, Halas NJ, Hartgerink JD (2008) Aromatic amino acids providing characteristic motifs in the Raman and SERS spectroscopy of peptides. J Phys Chem B 112:9158-9164

43. Niaura G, Gaigalas AK, Vilker VL (1997) Surface-enhanced Raman spectroscopy of phosphate anions: adsorption on silver, gold and copper electrodes. J Phys Chem B 101:9250-9262

44. Meier RJ, Csiszár A, Klumpp E (2006) On the interpretation of the $1100 \mathrm{~cm}^{-1}$ Raman band in phospholipids and other alkylcontaining molecular entities. J Phys Chem B 110:5842-5844

45. Bunding Lee KA (1989) Fourier transform infrared spectroscopic studies of microstructures formed from 1,2-bis(10,12-tricosadiynoyl)-sn-glycero-3-phosphocholine. J Phys Chem 93:926-931 